\title{
Experimental and Computational Evidence of non-MEP Pathways in the Fragmentation and Rearrangement of Bicyclo[3.3.1] heptane Diazonium Ions
}

\author{
Bastian Wulff ${ }^{\S}$, Patrick Sakrausky ${ }^{\#}$, Katrin Adamczyk ${ }^{\ddagger}$, Nils Huse ${ }^{\ddagger}$, Julia Rehbein ${ }^{\# *}$ \\ §Universität Hamburg, Fachbereich Chemie, Martin-Luther-King-Platz 6, 22761 Hamburg, Germany \\ ‡ Universität Hamburg, Institut für Nanostruktur- und Festkörperphysik, Luruper Chaussee 149, 22761 \\ Hamburg, Hamburg, Germany \\ \#Universität Regensburg, Fakultät für Chemie und Pharmazie, Universitätstrasse 30, 93051 \\ Regensburg, Germany
}

\begin{abstract}
Oxadiazoles have been utilized to release norbornyl-related carbocations by photoactivation in protic polar media. The product formation has been monitored by ${ }^{1} \mathrm{H}$ NMR and the experimentally observed product ratios were analyzed in comparison to expected product ratios based on minimum energy pathway calculations (MEP) and molecular dynamics simulations (MDs). Viewing all aspects together the results deliver the strong indication of non-MEP situations playing a major role in the product formation from fenchyl and bornyl diazonium ions. The valley-ridge inflection points have been further localized by projected frequency analysis. The here described observation supplements the computational studies by the groups of Tantillo as well as Weitman and Major in the closely related field of reaction path bifurcations in the rearrangements of norbornyl related carbo cations.
\end{abstract}

\section{Introduction.}

Secondary carbocations imbedded in bicyclo[2.2.1]heptane frameworks pose a longstanding fascination for physical organic chemists. The controversial discussion of their true electronic structure is well known. ${ }^{1}$ The now proven charge delocalization via sigma bonds leading to the term non-classical carbo cations shape not only the molecular structure but also their reactivity. ${ }^{2}$ For instance, the stabilization achieved by the charge delocalization within the norbornyl cation via the C6-C1 bond creates a driving force and enhances solvolysis rates of endo-isomers of norbornyl tosylates by factors of around $1000 .^{3}$ Besides these established characteristics, the discussion of a potential link between non-classical carbo cations and certain non-MEP reaction pathways like reaction path bifurcations (BIFs) is currently ongoing. Computational evidence has been provided mainly by the groups of Major ${ }^{4}$

\footnotetext{
${ }^{1}$ Robert A. Moss "The 2-norbornyl cation: a retrospective", J. Phys. Org. Chem. 2017, 27, 374-379

${ }^{2}$ Scholz, F.; Himmel, D.; Heinemann, F. W.; Schleyer, P. v. R.; Meyer, K.; Krossing, I. "Crystal Structure Determination of the Nonclassical 2-Norbornyl Cation", Science 2013, 341, 62 - 64.

${ }^{3}$ T. W. Bentley, C. T. Bowen, D. H. Morten, and Paul v. R. Schleyer J. Am. Chem. Soc. 1981, 103, 54665475.

${ }^{4}$ [a] Major, D. T.; Weitman, M., 'Electrostatically guided dynamics--the root of fidelity in a promiscuous terpene synthase?', J. Am. Chem. Soc. 2012, 134, 19454-19462 and references cited therein. [b] Weitman, M. Major, D. T., 'Challenges posed to bornyl diphosphate synthase: diverging reaction mechanisms in monoterpenes', J. Am. Chem. Soc. 2010, 132, 6349-6360.
} 
and Tantillo. ${ }^{5}$ However, a link to experimental data has not been established yet.

Here, we present experimental evidence for reaction path bifurcations being related to non-classical carbo cationic structures and their direct cationic precursors. The impact of subtle changes in the substitution pattern on the occurrence of BIFs is exemplified by carbo cations featuring fenchyl and bornyl frameworks.

To follow the onward reaction of rather short-lived species like carbo cations a photochemical release is mandatory. However, the photochemical generation of carbo cations is usually a stepwise process whereas a direct photon-induced heterolytic bond cleavage is mostly unknown. Instead homolytic bond cleavage yielding radicals as direct predecessors of the ion pair is a common mechanistic scenario. Mandatory for a clean 'ionic' follow-up chemistry is a fast single electron transfer (SET) due to strong spin-orbit coupling as for instance described for the heavy alkyl halogenides (iodides and bromides) ${ }^{6}$. An alternative access to carbo cations via photo activation starts with a charged precursor like phosphonium ${ }^{7}$ or diazonium salts ${ }^{8}$ (this work). The latter can be generated in situ via the photofragmentation of oxadiazoles to diazo alkanes (Scheme 1).

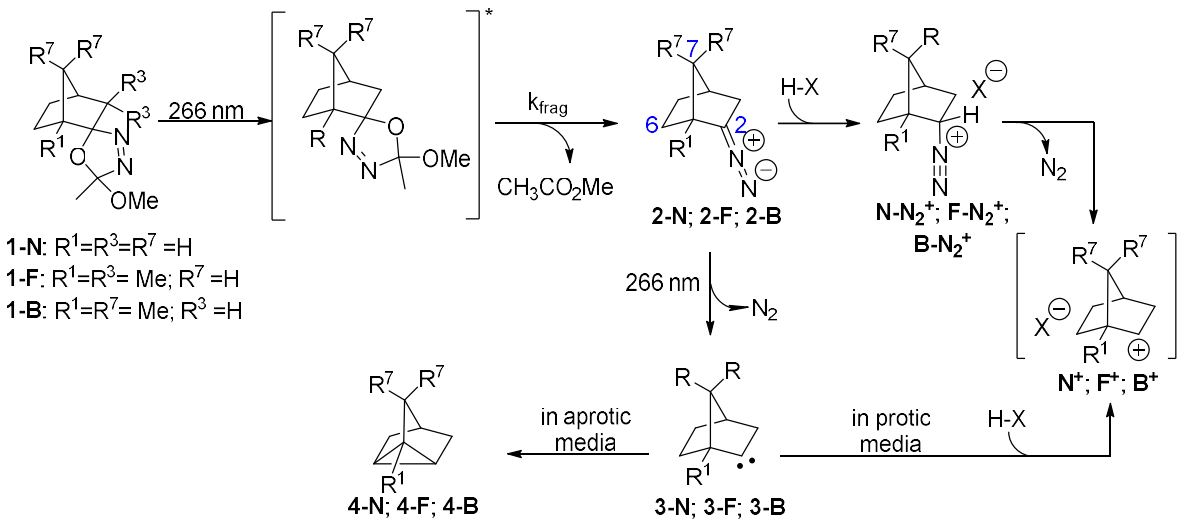

Scheme 1 Postulated mechanism of photo fragmentation leading to carbenes (in aprotic media) and to carbocations (protic media). The rate of fragmentation of 1-N to diazo $\mathbf{2 - N}$ has been determined in ACN and HFIP by time-resolved infrared spectroscopy (TRIR, see Figure 1).

The generation of the diazo alkane accompanied by release of acetyl methyl ester as the stoichiometric side product (Scheme 1) can be followed by time-resolved infrared spectroscopy (TRIR, Figure 1)): Femtosecond photo-excitation and subsequent probing of vibrational resonances probes the diazo

\footnotetext{
5 Recent reviews: [a] Hare, S. R.; Tantillo, D. J., ", Beilstein J. Org. Chem. 2016, 12, 377-390. [b] Tantillo, D. J. Chem. Soc. Rev. 2010, 39, 2847 - 2854. [c] Hong, Y J., Tantillo, D. J. Nature Chemistry 2009, 1, 384-389.

${ }^{6}$ Kropp, Paul J.; Adkins, Rick L., ,Photochemistry of alkyl halides. 12. Bromides vs. iodides ', J. Am. Chem. Soc. 1991, 113, 2709-2717.

7 [a] Ammer, J.; Sailer, C. F.; Riedle, E.; Mayr, H. 'Photolytic generation of benzhydryl cations and radicals from quaternary phosphonium salts: how highly reactive carbocations survive their first nanoseconds' J. Am. Chem. Soc. 2012, 134, 11481-11494. [b] Peranovich, T. M. S.; Marcondes, M. E. R.; Toscano, V. G. 'Photochemistry of Phosphonium Salts-Photolysis of 9-(Antryl)Methyl Triphenylphosphonium Chloride' Phosphorus, Sulfur, and Silicon and the Related Elements 1990, 54, 314.

8 [a] John Paul Pezacki, Brian D. Wagner, Calvin S. Q. Lew, John Warkentin, and Janusz Lusztyk, ' $\Delta^{3}-1,3,4$ Oxadiazolines: Photochemical Precursors to Diazoalkanes and sec-Alkanediazonium Ions in Acidic Solution', J. Am. Chem. Soc. 1997, 119, 1789 - 1790. [b] John Paul Pezacki, Deepak Shukla, Janusz Lusztyk, and John Warkentinm 'Lifetimes of Dialkylcarbocations Derived from Alkanediazonium Ions in Solution: Cyclohexadienyl Cations as Kinetic Probes for Cation Reactivity' J. Am. Chem. Soc. 1999, 121, 6589 - 6598.
} 
alkane and the methyl ester which exhibit characteristic stretching vibrations of the diazo and the carbonyl group, respectively (Fig. 1A). The formation of these vibrational resonances is occurring on picosecond-timescales (Fig. 1B,C; see SI for experimental details). While intramolecular vibrational energy relaxation (IVR) and solvation influence the absorption strength at early times, the formation time constants of the spectroscopic signals provide an upper bound for the fragmentation process which is complete at 20 ps after photo-excitation. Product absorption thermalizes on tens of picoseconds and reaches a constant level after $\sim 60$ ps in acetonitrile (ACN) and in hexafluoroisopropanol (HFIP) after $\sim 100$ ps. The absence of signal decay at 200 ps delay suggests a primary product lifetime of at least $1 \mathrm{~ns}$.
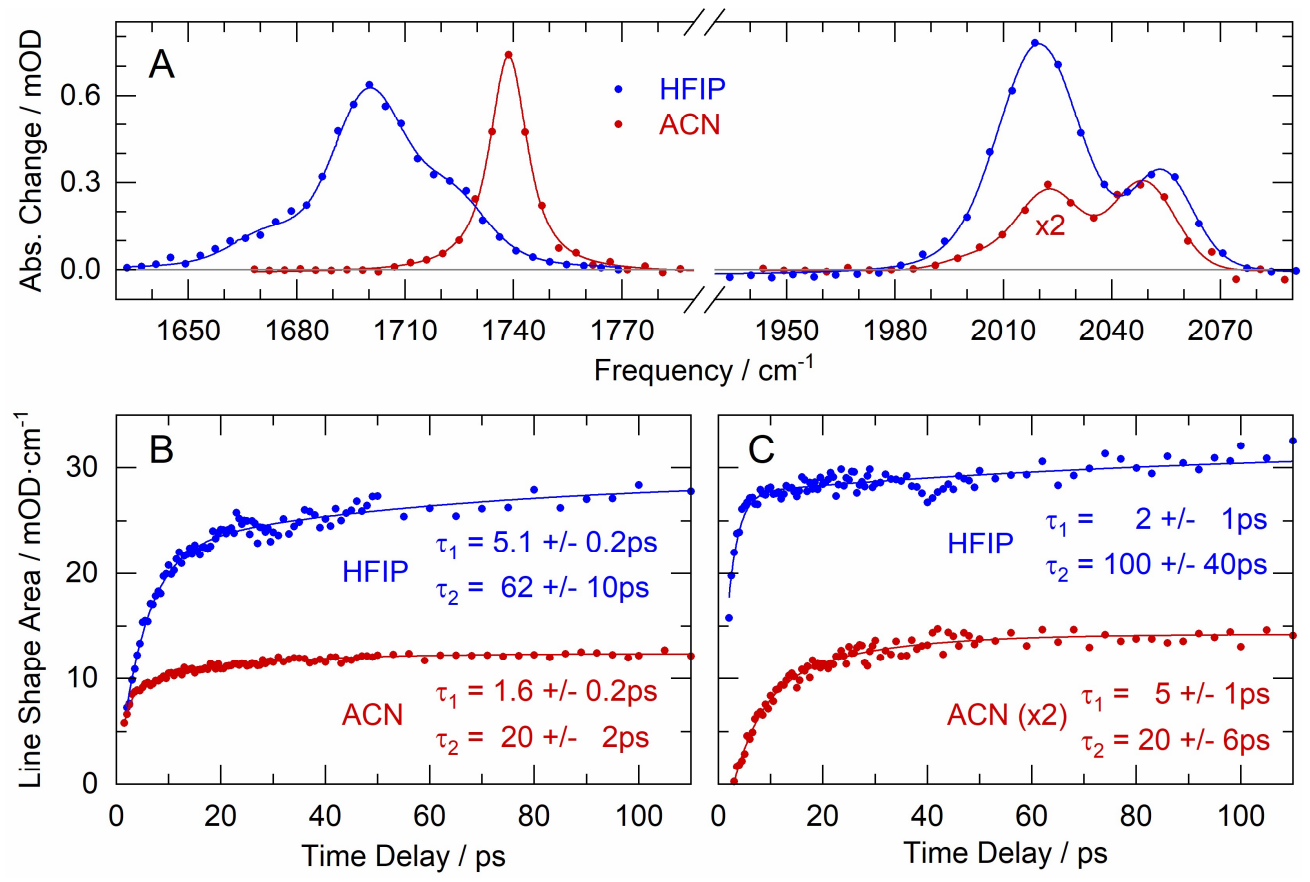

Figure 1 Results of femtosecond infrared spectroscopy with 267-nm excitation of 1-N in HFIP and ACN monitoring the formation of 2-N. Absorption lineshapes of the carbonyl of the acetyl methyl ester and the diazo stretching vibration $60 \mathrm{ps}$ of 2-N after 267-nm excitation (A). Time evolution of the lineshape area the carbonyl (B) and diazo (C) stretching vibrations in HFIP (blue) and ACN (red) solution. The lines are fits of a sequential rate equation model (see supporting information for details).

In protic polar media (alcohols, acidic additives) diazo alkanes can be protonated to yield diazonium salts that are believed to fragment spontaneously to carbo cations and dinitrogen. The advantage of oxadiazole in the mechanistic study of carbo cations is the irreversible formation of the desired product as re-trapping by the leaving group is inhibited $\left(\mathrm{N}_{2}\right)$. In addition, the potential impact on differences in reactivity of different diastereomers is levelled by the long lifetime of the diazo-intermediate. Also, the oxadiazoles as such are stable compounds (storage at $-20^{\circ} \mathrm{C}$ for month) and synthesized easily in two steps.

Extensive computational analysis of the underlying potential energy surfaces for the diazonium ion fragmentation and subsequent skeletal rearrangements of the fenchyl and bornyl frameworks complete the picture and provide a rational for the observed product ratios. 
Methods. Oxadiazolines of norbornanone, fenchone and norcamphor were prepared according to Warkentin and co-worker. ${ }^{9}$ Endo- and exo-diastereomers of $\mathbf{N}$ (Table 1 ) were separated by solvolysis in wet ACN with 0.8 eq TFA, leaving one set of endo-diastereomers, $\mathbf{B}$ and $\mathbf{F}$ were used as the original diastereomeric mixture, since kinetics of the photochemical fragmentation confirmed same rate constants for all diastereomers.

Analysis of product ratios was done in two different approaches: on a preparative scale, reactions were run in de-aerated quartz cuvettes and on an analytical scale in quartz NMR tubes equipped with a Young's tap using deuterated solvents. Reaction solutions were irradiated with UVB lamps ( 6 Watt, 12 lamps) in a Rayonett reactor for different time intervals and subsequently analyzed by ${ }^{1} \mathrm{H} N M R$. To enable an unambiguous assignment of structures to spectral features a set of reference substance were synthesized.

Computational Details. All calculations presented herein were performed with the Gaussian09.D02 and .E02 program package. ${ }^{10}$ Geometry optimisations of stationary points were performed using different density functionals without (B3LYP, M062X $)^{11},{ }^{12}$ and with short-range dispersion correction in conjunction with the $6-31 G^{*}, 6-31+G(d, p)$ or $6-311+G^{* *}$ basis sets ${ }^{13}$. The choice of level of theory was guided by latest publications on related structures. ${ }^{14}$ Further details on the progression and initial conditions of the BOMD simulations can be found in the SI.

\section{Results \& Discussion.}

Product scope/ kinetics. In acetonitrile (ACN) the diazo fragmentation proceeds via the carbene pathway to form tricyclene (4-N, blue box, Figure 2) or its methylated derivatives (4-F, 4-B) as one of the main products. The diazo compounds $\mathbf{2}$ are unusually stable (calculated barrier of thermally active $\mathrm{N}_{2}$-extrusion: $\Delta \mathrm{G}^{\ddagger}{ }_{\mathrm{N}}=28.9 \mathrm{kcal} / \mathrm{mol}$, see $\mathrm{SI}$ for TRIR data) and can be observed by ${ }^{1} \mathrm{H}$ NMR-spectroscopy of irradiated samples in $\mathrm{ACN}$. Subsequent optimization of the reaction conditions led to the favorite choice of HFIP as solvent and proton source. ${ }^{15} \mathrm{Here}$, the diazo protonation became competitive to the de-azatation of the diazo 2-N (ratio 2 : 1 ionic : carbene path) or even the unique reaction path (2-F, 2B). According to the calculated barrier for $\mathrm{N}_{2}$-extrusion out of the diazoniumion the process is now barrier-free $\left(\Delta \mathrm{G}^{\ddagger}\right.$ endo-N-N2+$=1.3 \mathrm{kcal} / \mathrm{mol} ; \Delta \mathrm{G}^{\ddagger}$ exo-N-N2+$\left.^{-}=2.1 \mathrm{kcal} / \mathrm{mol}\right)$.

\footnotetext{
${ }^{9}$ Bekhazi, M.; Risbood, P. A.; Warkentin, J. J. Am. Chem. Soc. 1983, 105 (17), 5675-5679.

${ }^{10}$ Gaussian09.D02 \& E02 Version, for full citation see SI.

${ }^{11}$ B3: Becke, A. D. J. Chem. Phys. 1993, 98, 5648; LYP: Lee, C.; Yang, W.; Parr, R. G. Phys. Rev. B 1988, $37,785$.

12 Zhao, Y., Truhlar, D.G Theor Chem Account 2008, 120, 215-241.

13 6-31G: [a] Ditchfield, R;. Hehre, W. J.; Pople, J. A. J. Chem. Phys. 1971, 54, 724; 6-311: [b] Raghavachari, K.; Binkley, J. S.; Seeger, R.; Pople J. A. J. Chem. Phys. 1980, 72, 650-54. [c] McLean, A. D.; Chandler, G. S. J. Chem. Phys. 1980, 72, 5639-48.

14 [a] Hong, Y., Tantillo, D. Biosynthetic consequences of multiple sequential post-transition-state bifurcations. Nature Chem 6, 104-111 (2014). [b] Y. J. Hong, D. J. Tantillo, 'Quantum chemical dissection of the classic terpinyl/pinyl/bornyl/camphyl cation conundrum - the role of pyrophosphate in manipulating pathways to monoterpenes', Org. Biomol. Chem. 2010, 8, $4589-4600$.

${ }^{15}$ See SI: Addition of TFA (0.2 - 16 eq) to a solution of $\mathbf{N}$ in ACN-d 3 should lead to a protonation of the diazo intermediate, but according to the product ratios $(1: 1)$ the ionic pathway (red \& grey boxes. Table 1 ) is not yet favored over the carbene path.
} 


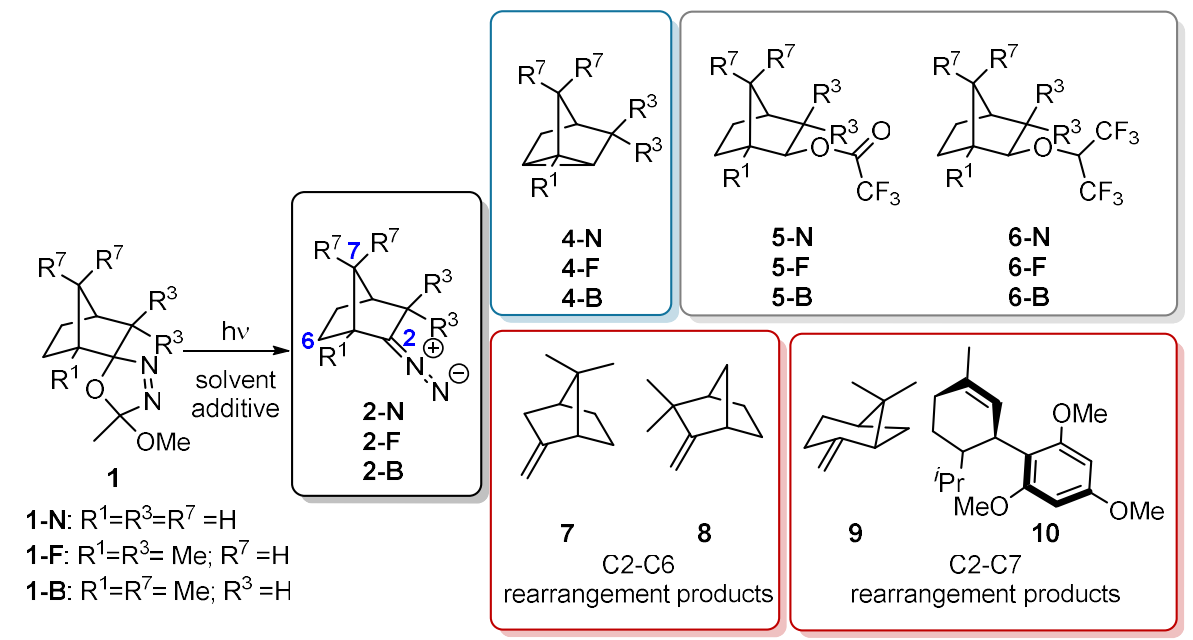

Figure 1 Experimentally observed products under continuous irradiation (UVB, Rayonett, quartz NMR tube, 60 min): 2, 4 major products in ACN, 2, 4, 5 in ACN with 1 eq TFA, 6-8 major products in HFIP and 6-10 major products in HFIP with 10 eq TMB.

The fragmentation of the heterocycle and formation of the different terpenoid structures occurs quantitatively based on the occurrence of $91-98 \%$ of the stoichiometric side product methyl acetate $\left({ }^{1} \mathrm{H}\right.$ NMR data \& TRIR data) and is independent of the solvent and protic additives within the error margin of the measured rate constants. Moreover, the different diastereomers are consumed with similar rate constants. Ps-time-resolved IR spectroscopy (ps-TRIR) could show that $\mathbf{2}$ is formed within $2 \mathrm{ps}$ and hence presumably within one step after the electronic excitation and that $\mathbf{2}$ is a rather longlived species even in HFIP (minimum life time of $50 \mathrm{ps}$ ). ${ }^{16}$ Hence, the chemistry related to the carbo cation formation out of the diazo compounds is independent of the configuration of the starting materials $\mathbf{N}, \mathbf{B}$, and $\mathbf{F}$.

With fenchyl and bornyl carbon frameworks rearrangement products are expected to occur and indeed are observed in form of the corresponding alkenes or the TMB-trapping ${ }^{17}$ product (Scheme 2 ). The formation of the rearrangement products is on average at $61 \%$ independent of the presence of TMB. ${ }^{18}$

Table 1 Summary of in situ-NMR studies of product ratios (C2-C6 products: trapping or elimination products of $\boldsymbol{\alpha}^{-\mathrm{F}^{+}}$or $\mathrm{C}^{+}$; C2-C7 products: trapping or elimination products of $\left.\mathrm{P}^{+}, \mathrm{T}^{+}, \mathbf{P h e}^{+}\right)$. See Schemes 2 and 3 for structures.

\begin{tabular}{ccccccc}
\hline$\#$ & prec. & TMB & $\begin{array}{c}\text { C2-C6 } \\
\text { products } \\
{[\%]^{a}}\end{array}$ & $\begin{array}{c}\text { C2-C7 } \\
\text { products } \\
\text { [\%] }^{a}\end{array}$ & $\begin{array}{c}\text { sum } \\
{[\%]}\end{array}$ & $\begin{array}{c}\text { ratio } \\
\text { C2-C6 : } \\
\text { C2-C7 }\end{array}$ \\
\hline 1 & 1-F & - & 54 & 5 & 59 & $11: 1$ \\
2 & 1-B & - & 56 & 0 & 56 & $\begin{array}{c}\text { Quant. } \\
\text { C2-C6 }\end{array}$ \\
3 & 1-F & 4 eq & 49 & 22 & 61 & $2: 1$ \\
4 & 1-B & 4 eq & 61 & 5 & 66 & $12: 1$ \\
\hline
\end{tabular}

${ }^{a}$ Measured in HFIP- $d 2$ under irradiation with UVB-lamps in quartz NMR-tubes under inert atmosphere.

\footnotetext{
${ }^{16}$ For further details and results of kinetics and transient absorption spectroscopy see $\mathrm{SI}$.

${ }^{17}$ TMB-trapping: Steenken, S.; Ashokkumar, M.; Maruthamuthu, P.; McClelland, R. A., , Making Photochemically Generated Phenyl Cations Visible by Addition to Aromatics: Production of Phenylcyclohexadienyl Cations and Their Reactions with Bases/Nucleophiles', J. Am. Chem. Soc. 1998, 120, 11925-11931.

${ }^{18}$ The remaining $39 \%$ could not be identified.
} 
Two experimental observations can be made: First, in general the $\mathrm{C} 2-\mathrm{C} 6$ rearrangement products are preferred over $\mathbf{C 2}-\mathrm{C} 7$ ones for both $\mathbf{F}$ and $\mathbf{B}$, independent of the absence or presence of TMB and with $\mathbf{F}$ is providing lower selectivities than $\mathbf{B}$. Second, in presence of TMB one sees for both $\mathbf{F}$ and $\mathbf{B}$ higher amounts of the $\mathrm{C2}-\mathrm{C} 7$ rearrangement products than without TMB, i.e. a decrease in C2-C6-selectivity. The latter observation is quite likely due to the trapping of the phenandryl cation $\mathbf{P h e}^{+}$by the very good nucleophile TMB (Scheme 2 and 3) before it can revert back to the thermodynamic sink of the PES, i.e. $\boldsymbol{\alpha}-\mathbf{F}^{+}$or $\mathbf{C}^{+}$(C2-C6 products). This assumption is corroborated by the relative barrier heights of the associated elementary steps of the cationic rearrangements summarized in Scheme $3 .{ }^{19}$

To find a rational of the experimentally determined selectivities the reaction coordinates of the dissociation of the endo- and exo-isomers of the $\mathbf{B}-\mathbf{N}_{2}{ }^{+}$and the $\mathbf{F}-\mathbf{N}_{2}{ }^{+}$have been analyzed in detail (Scheme 2).

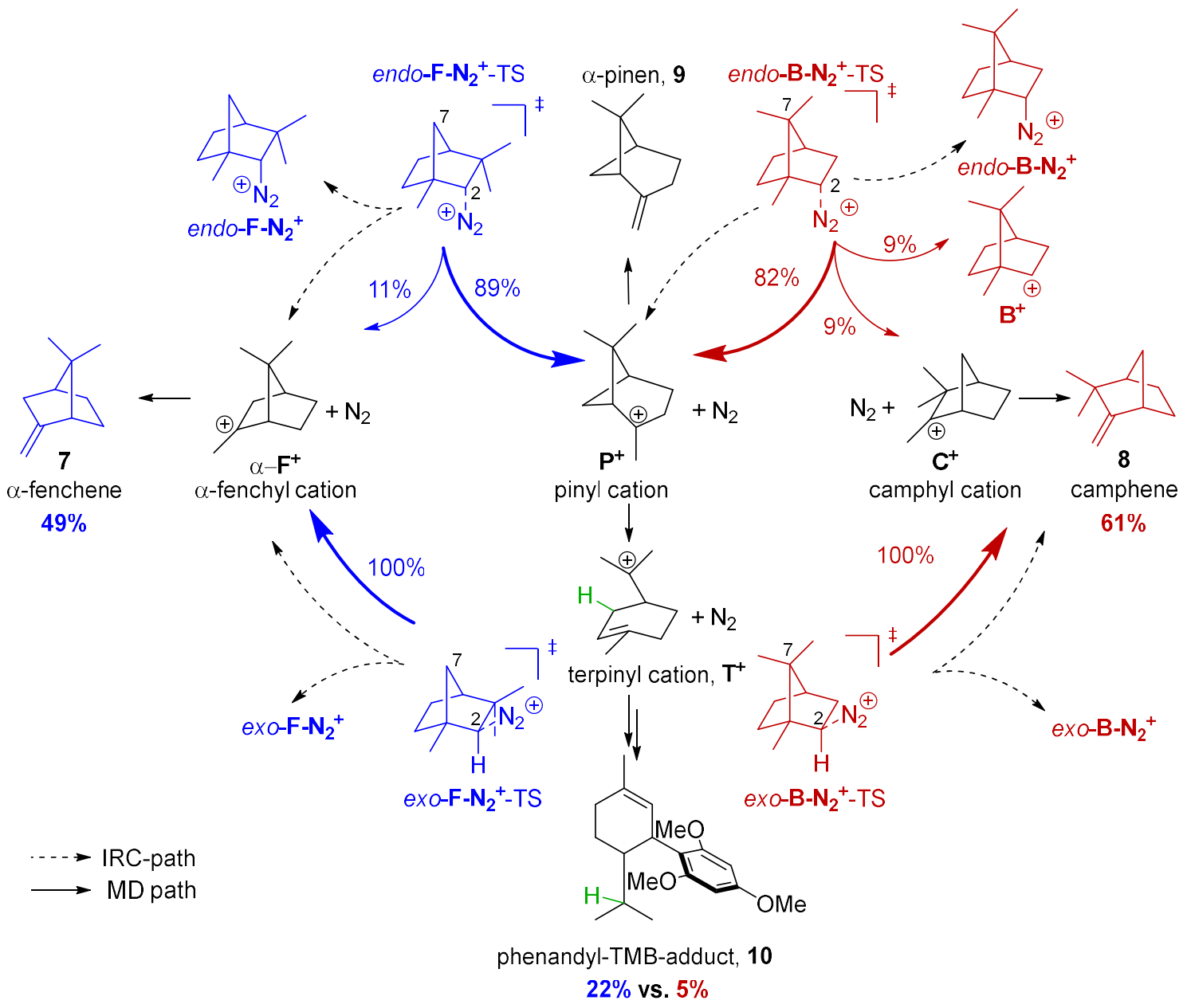

Scheme 2 Summary of product ratios based on MEP-pathways \& MD-Pathways (\% numbers) and experimentally observed product selectivity (bold \% numbers) for the fragmentation and rearrangement of diazonium ions in dependence of their endo- or exo-configuration.

\footnotetext{
${ }^{19}$ That indeed the carbocation is trapped by TMB was shown by the transient absorption spectrum of the corresponding cyclohexadienylcation using a ns-pump-prove set-up. See SI for the transient absorption spectrum.
} 
The IRC-product in case of both endo-and exo-isomers of $\mathbf{F}-\mathrm{N}_{2}{ }^{+}$is the $\mathrm{C} 2-\mathrm{C} 6$ rearrangement product, i.e. the $\alpha$-fenchyl cation, $\boldsymbol{\alpha}-\mathbf{F}^{+}$(Scheme 2, left side).

In case of $\mathbf{B}-\mathbf{N}_{\mathbf{2}}{ }^{+}$the IRC products depend on the configuration at $\mathbf{C 2}$ : endo-B- $\mathbf{N}_{\mathbf{2}}{ }^{+}$is linked directly to the $\mathrm{C} 2-\mathrm{C7}$ rearrangement product, i.e. the pinyl cation, $\mathbf{P}^{+}$, whereas the exo-B- $\mathbf{N}_{2}{ }^{+}$leads to $\mathbf{C 2}-\mathbf{C 6}$ rearrangement, i.e. camphyl cation $\mathbf{C}^{+}$(Scheme 2 , right side).

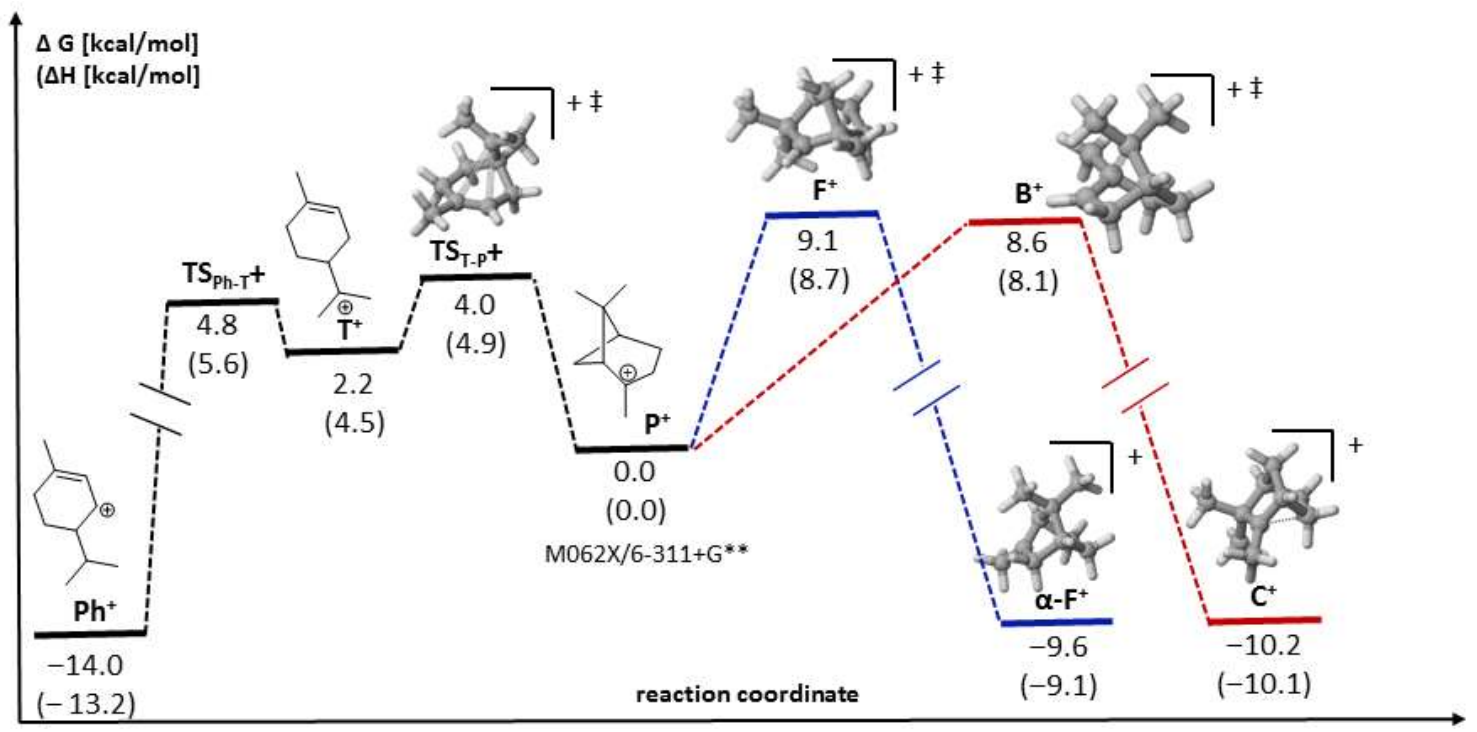

Scheme 3 Reaction coordinate diagram of the free carbocations in black the common intermediates of both branches of $\mathbf{F}^{+}$ (blue lines) and $\mathbf{B}^{+}$(red lines). The preceding steps (black line) are identical for both isomers $\mathbf{F}^{+}$and $\mathbf{B}^{+}$. The data shown was calculated at M06-2X/6-311+G**.

Based on the IRC calculations the expectation would be that fragmentation of either endo- or exo-F$\mathbf{N}_{2}{ }^{+}$would lead to $\boldsymbol{\alpha}-\mathbf{F}^{+}$selectively and hence one would observe in the NMR the corresponding elimination product $\alpha$-fenchen or trapping products (HFIP ether, TMB adduct). This expectation is only valid of course, as long as the barrier $(18.7 \mathrm{kcal} / \mathrm{mol})$ for reverting back to the pinyl cation is higher than the barriers for elimination or trapping. This can be assumed with some confidence as HFIP though not a good nucleophile - is abundant as a solvent and secondly the alcoholate stays in close proximity (ion pair) after proton transfer, so both formation of the alkene or the ether within the ion pair is facilitated and not slowed down by diffusion. ${ }^{20}$ In presence of TMB, this assumption is even more reliable as TMB is a very good nucleophile and captures cations with rate constants close to the diffusion controlled limit. ${ }^{17}$

However, the MEP prediction of only $\boldsymbol{\alpha}-\mathbf{F}^{+}$derivatives (C2-C6 rearrangement products, Table 1 ) is an obvious contradiction to the experimental observation of $11: 1 \mathrm{C} 2-\mathrm{C} 6: \mathrm{C} 2-\mathrm{C} 7$ (without TMB) or $2: 1$ (with TMB) product ratios. ${ }^{21}$ Based on the special nature of the closely related fenchyl cation $\mathrm{F}^{+}$, being part of a bifurcating PES (Scheme 3) we therefore analyzed also the molecular dynamics of the $\mathbf{F}-\mathbf{N}_{2}{ }^{+}-$ fragmentation in respect to off-MEP behavior (vide infra).

The IRC analysis of the $\mathbf{B}-\mathbf{N}_{2}{ }^{+}$delivered a more complicated picture (Scheme 2, right): Here, the exoisomer leads via the MEP to $\mathbf{C}^{+}$and similarly to the case of $\alpha-\mathbf{F}^{+}, \mathbf{C}^{+}$can be assumed to be formed irreversibly under the reaction conditions. Hence, for $100 \%$ exo-B- $\mathbf{N}_{2}{ }^{+}$one would expect to see only

\footnotetext{
${ }^{20}$ This is based on a) MD simulations with HOAc that protonation and subsequent trapping or deprotonation by the acetate as a close to barrier-free process. B) preliminary in situ NMR studies indicating significant and specific $\mathrm{H}$-bonding between HFIP and precursor 1.

${ }^{21}$ It was ensured that the final products are not photochemically active under irradiation with UVB light.
} 
camphene or trapping products of $\mathbf{C}^{+}$(C2-C6 product). On the contrary, the endo-B- $\mathbf{N}_{2}{ }^{+}$leads to the pinyl cation $\mathbf{P}^{+}$as the IRC-product. Comparing the barriers around $\mathbf{P}^{+}$(Scheme 3 ) it is apparent that the formation of the terpinyl cation $\mathrm{T}^{+}$and onwards to the phenandyl cation, $\mathrm{Ph}^{+}$is almost a barrier-free process $\left(\Delta \mathrm{G}_{\mathrm{T}-\mathrm{P}}{ }^{\ddagger}=4.0 \mathrm{kcal} / \mathrm{mol}\right.$ and $\left.\Delta \mathrm{G}_{\mathrm{T}-\mathrm{ph}}{ }^{\ddagger}=2.6 \mathrm{kcal} / \mathrm{mol}\right)$. Since $\mathbf{P h}^{+}$is significantly more stable than $\mathbf{P}^{+}(-$ $14.0 \mathrm{kcal} / \mathrm{mol}, \Delta \mathrm{G}$ ) the lifetime should be sufficiently long to allow for the trapping by TMB in the experiment. The barriers towards the camphyl cation $\left(\mathbf{C}^{+}\right)$on the one hand and the $\alpha$-fenchyl cation $\left(\alpha-F^{+}\right)$on the other are somewhat higher, but still low $(8.1 \mathrm{kcal} / \mathrm{mol}$ and $9.6 \mathrm{kcal} / \mathrm{mol}$ respectively $)$ and accessible under the reaction conditions.

Again, the IRC-prediction of the product ratio is different to the experimental one. However, since for the bornyl-framework the MEP-selectivity is dependent on the endo-/exo-configuration of the diazoniumion, and this very ratio can only be predicted based on computed relative stabilities (2:1 in favour of the endo-configuration), the following discussion of the MD results will focus mainly on the fenchyl-system. BOMD simulations were started at the transition state of the $\mathrm{N}_{2}$-extrustion of either endo- or exo-isomers of $\mathbf{F}_{-} \mathbf{N}_{2}{ }^{+}$and $\mathbf{B}-\mathbf{N}_{2}{ }^{+}$and the results are summarized in Table 2. Based on the relative stabilities of endo-vs. exo-isomers one can calculate the numbers of expected product ratios for a mixture of endo- and exo- $\mathbf{F}-\mathbf{N}_{2}{ }^{+}$and $\mathbf{B}-\mathbf{N}_{2}{ }^{+}$.

Table 2 Comparison of predicted product ratios and experimentally observed ones.

\begin{tabular}{|c|c|c|c|c|c|c|}
\hline \# & & $\begin{array}{c}\mathrm{MD} \\
\mathrm{C} 2-\mathrm{C} 6 \text { vs. } \\
\mathrm{C} 2-\mathrm{C} 7\end{array}$ & $\begin{array}{c}\mathrm{MD} \text { rel. stab. } \\
\mathrm{C} 2-\mathrm{C} 6 \text { vs. } \\
\mathrm{C} 2-\mathrm{C} 7\end{array}$ & $\begin{array}{c}\text { MEP } \\
\text { C2-C6 vs. } \\
\text { C2-C7 }\end{array}$ & $\begin{array}{c}\text { MEP } \text { rel. stab. } \\
\text { C2-C6 vs. } \\
\text { C2-C7 }\end{array}$ & $\begin{array}{c}\text { exp. }^{c} \\
\text { C2-C6 vs. } \\
\text { C2-C7 }\end{array}$ \\
\hline 1 & exo-F-N ${ }_{2}^{+}$ & 1:0 & $2 \cdot 1^{a}$ & 1:0 & $1 \cdot 0^{a}$ & $2 \cdot 1$ \\
\hline 2 & endo-F- $\mathbf{N}_{\mathbf{2}}^{+}$ & $1: 8$ & 2.1 & $1: 0$ & 1.0 & 2.1 \\
\hline 3 & exo-B- $\mathrm{N}_{2}^{+}$ & 1:0 & $1: 2.0^{b}$ & $1: 0$ & $1: 2.3^{b}$ & $12: 1$ \\
\hline
\end{tabular}

${ }^{c}$ relative stability endo- vs. exo-isomer: $+0.3 \mathrm{kcal} / \mathrm{mol}$, i.e. endo $:$ exo $=1: 1.7 .^{b}$ relative stability endo- vs. exoisomer: $-0.5 \mathrm{kcal} / \mathrm{mol}$, i.e. endo : exo $=2.3: 1 .^{c}$ with 4 eq TMB.

By comparison of the MD- predicted product ratios ( $\mathrm{C} 2-\mathrm{C} 6$ vs $\mathrm{C2}-\mathrm{C} 7$ ) with the experimental ones, one can see, that the numbers fit amazingly well for the fenchyl system $\mathbf{F}-\mathbf{N}_{2}{ }^{+}$whereas the MEP-predictions were suggesting $100 \%$ formation of C2-C6 products only.

For the bornyl-case the results are not matching exactly, but even though the absolute numbers do not fit, the MD simulations show a deterioration of the predicted MEP-selectivities towards the observed preference of $\mathrm{C2}-\mathrm{C} 6$ products. Overall, the reduced selectivities as observed in the MDS are down to the trajectories that bifurcate to the non-MEP-products (Table 1, Scheme 2) and thereby provide a rational for the lower than expected experimental product selectivity.

To further test the hypothesis of a reaction path bifurcation we aimed at a better understanding of the topology of the PES describing the fragmentation and skeletal rearrangements. Therefore, relaxed scans of the PESs of the diazoniumions were conducted, driving the geometric parameters $r\left(C 2-\mathrm{N}_{2}\right)$ (fragmentation) and $r(\mathrm{C} 2-\mathrm{C} 6)$ simultaneously. As a third computational analysis tool for identifying valley ridge inflection points - the prerequisite for reaction path bifurcations - the frequencies of structures along the IRC associated to $\mathbf{F}-\mathbf{N}_{2}{ }^{+}-\mathbf{T S}$ and $\mathbf{B}-\mathbf{N}_{2}{ }^{+}-\mathbf{T S}$ were projected and analyzed. This approach, though being again a PES analysis avoids the bias introduced by scanning the PES by using simple specified reaction coordinates.

The endo-F- $\mathbf{N}_{2}{ }^{+}$ion is part of a PES that clearly features a bifurcation if plotted as shown in Figure 2 . The endo-F- $\mathbf{N}_{2}{ }^{+}$-TS (fragmentation TS) is associated with the MEP towards the fenchyl cation $\mathbf{F}^{+}$that resides close to the valley-ridge inflection point (VRI). The $2^{\text {nd }}$ transition state belongs to the 
interconversion of the pinyl cation $\left(\mathbf{P}^{+}\right)$and the $\alpha-\mathbf{F}^{+}$. The structure-related analysis of the BOMD simulations revealed that the trajectories diverge in proximity to the diazoniumion-fragmentation TS and either led to the $\boldsymbol{\alpha}-\mathbf{F}^{+}$(major path) or to $\mathbf{P}^{+}$without any re-crossing and no interconversion between $\alpha-\mathbf{F}^{+}$and $\mathbf{P}^{+}$on the simulation time scales (Scheme 2 , top left).
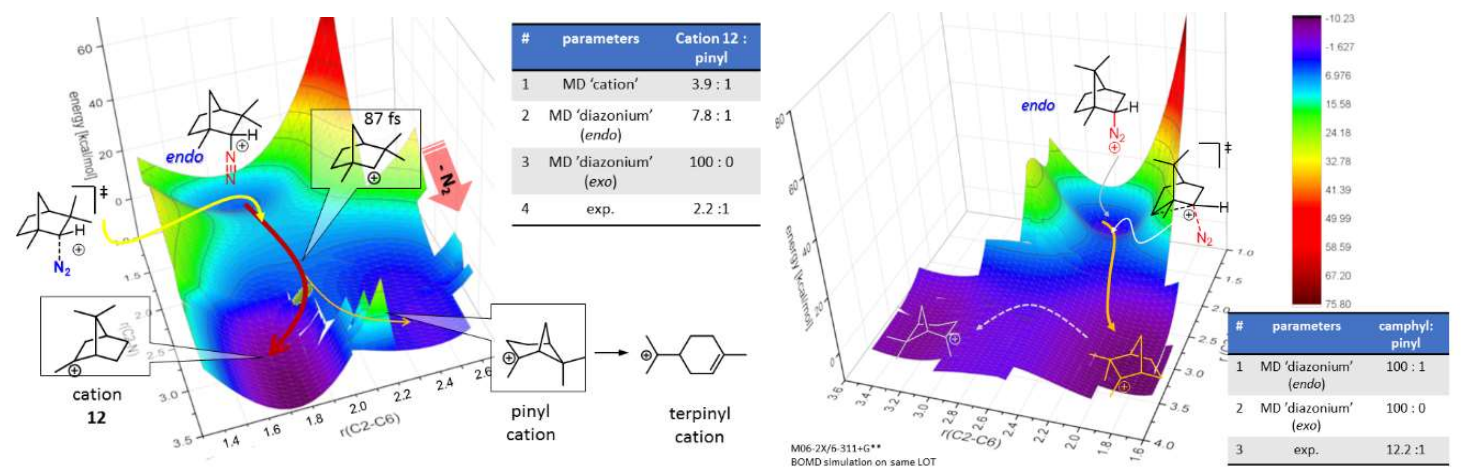

Figure $23 \mathrm{D}$ plots of the PESs scans gerated by driving the $\mathrm{N}_{2}-\mathrm{C}$ bond length and the $\mathrm{C} 2-\mathrm{C} 6$ bond length simulataneoulsy.

The additional analysis by using projected frequencies support the BOMD results and allowed for a better structural characterization of the valley-ridge inflection points (VRT-I and VRT-II, Figure 4). The VRI point is defined by the change in sign of the projected frequency from positive to negative. Only VRT-I is of importance for the skeletal rearrangement of the carbon framework as the imaginary frequency here shows the necessary wagging movement to shorten the C2-C6 distance. VRT-II on the other hand only shows the rotation of the $\mathrm{N}_{2}$ in relation to the carbocation.

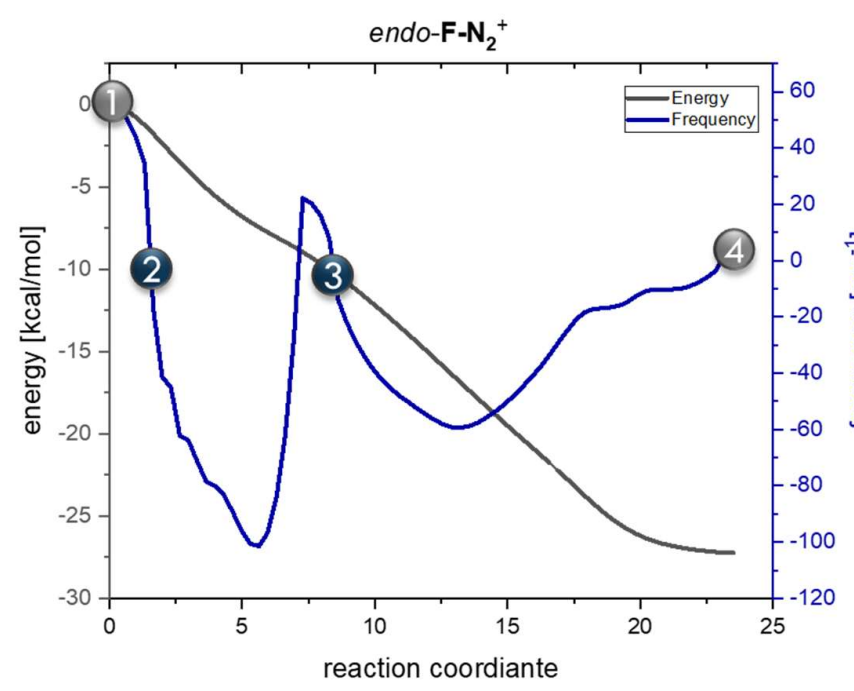

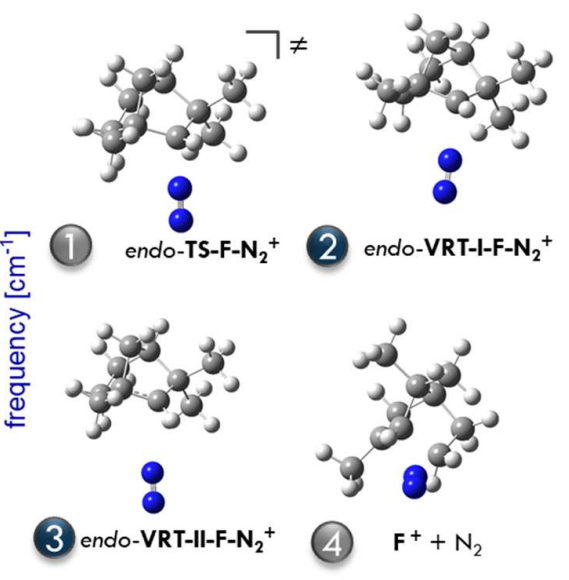



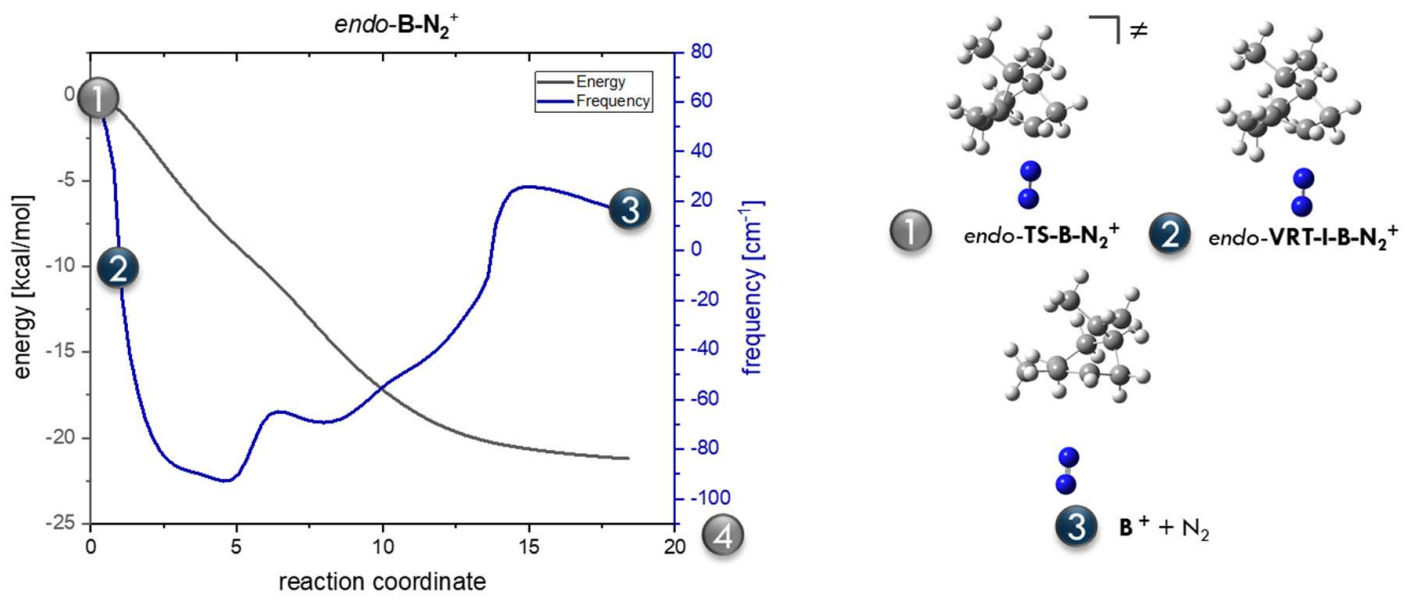

Figure 3 The projected frequencies along the IRC for endo-F- $\mathbf{N}_{2}{ }^{+}$(top) and endo-B- $\mathbf{N}_{2}{ }^{+}$(bottom) indicate that close by the TS of the $\mathrm{N} 2$ release there is a valley ridge inflection point (VRT).

Changing the stereochemistry of the $\mathbf{F}-\mathbf{N}_{2}{ }^{+}$from endo- to exo-isomer provides a very different picture. Here a clear MEP towards the $\alpha$-fenchyl (cation 12) is present. This MD simulations led quantitatively to the $\boldsymbol{\alpha}-\mathbf{F}^{+}$Table 2) and also the frequency analysis along the IRC-path indicate 'standard' MEP behavior (see SI for details).

As it became apparent from the analysis above, the observed product ratios in the bornyl framework are only qualitatively described correctly by the MDs simulations as the these predict a diminished selectivity. Again, the exo-B- $\mathbf{N}_{2}{ }^{+}-$like the exo-F- $\mathbf{N}_{2}{ }^{+}-$seems to follow the MEP, whereas the endoisomer shows interesting deviations from it. Although the scanned PES (Figure $3 b$ ) indicates rather MEP behavior the projected frequencies along the IRC suggest a BIF possible based on the existence of a VRI (Figure 4).

Conclusion. The combination of experimental determination of product ratios, spectroscopic characterization of intermediates by NMR, TRIR and TRUV on time-scales from min to ps and an indepth computational analysis beyond stationary point calculations and minimum energy pathways, led to the identification of reaction path bifurcations and the characterization of the associated valley ridge-inflection points in the fragmentation und rearrangement processes of bicyclic-diazonium ions. It was shown that the configuration of the diazonium ions (endo-vs. exo) determines the presence or absence of VRI points and thereby the impact of dynamically controlled product selectivity. The control of the diazoniumion configuration may hence in future deliver a handle to invoke the dynamic control at will and steer the product selectivity to the desired product. 


\section{Supporting Information}

\section{Experimental and Computational Evidence of non-MEP Pathways in the Fragmentation and Rearrangement of Bicyclo[3.3.1] heptane Diazonium lons}

Bastian Wulff ${ }^{\S}$, Patrick Sakrausky ${ }^{\#}$, Katrin Adamczyk ${ }^{\ddagger}$, Nils Huse ${ }^{\ddagger}$, Julia Rehbein ${ }^{\# *}$

§Universität Hamburg, Fachbereich Chemie, Martin-Luther-King-Platz 6, 22761 Hamburg, Germany

‡ Universität Hamburg, Institut für Nanostruktur- und Festkörperphysik, Luruper Chaussee 149, 22761

Hamburg, Hamburg, Germany

\#Universität Regensburg, Fakultät für Chemie und Pharmazie, Universitätstrasse 30, 93051 Regensburg, Germany

\section{Content}

Supporting Information

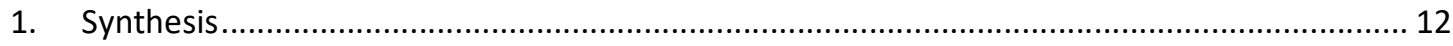

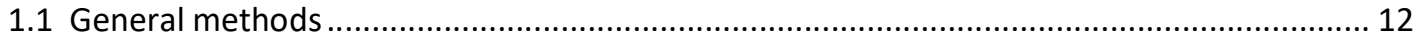

1.2 General procedure for the synthesis of $N$-acetyl hydrazones............................................. 12

1.3 General procedure for the synthesis of 2-methoxy-2-methyl- $\Delta^{3}-1,3,4$-oxadiazolines ........... 13

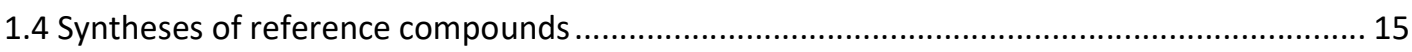

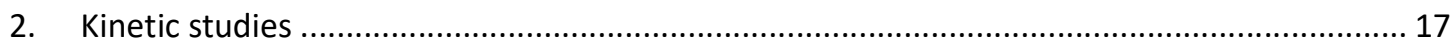

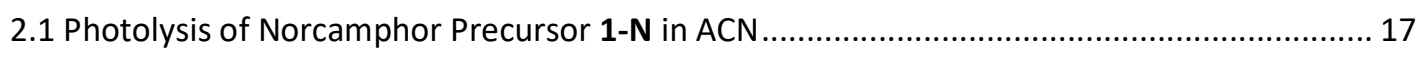

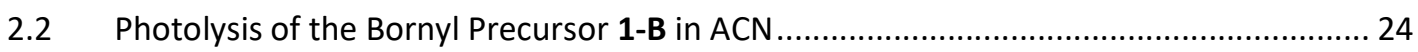

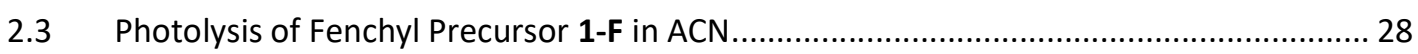

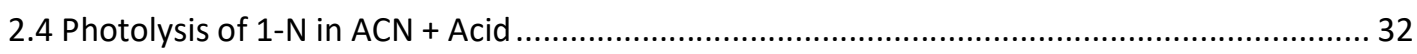

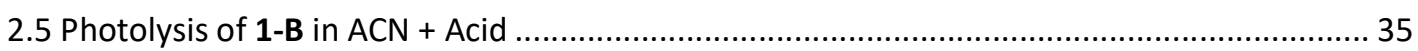

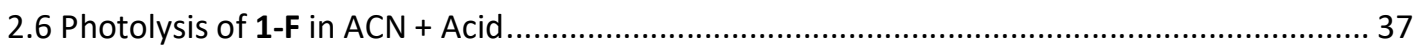

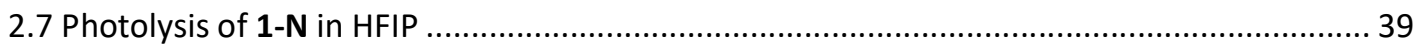

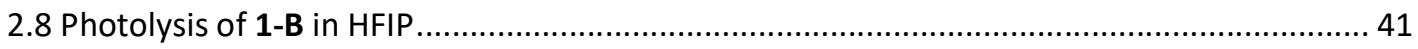

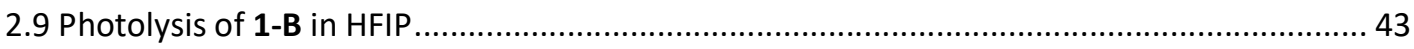

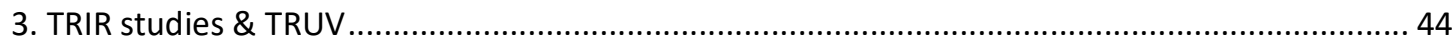

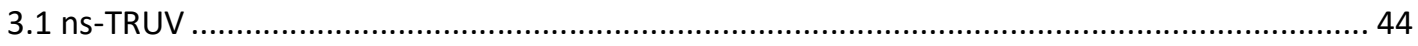

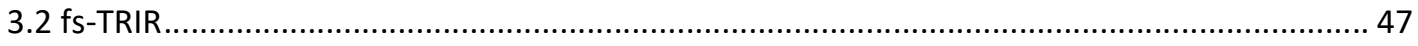

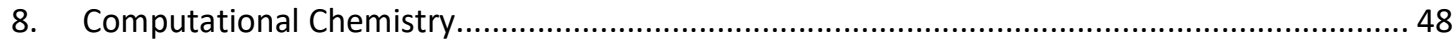

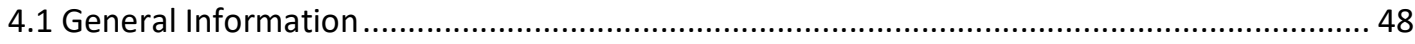

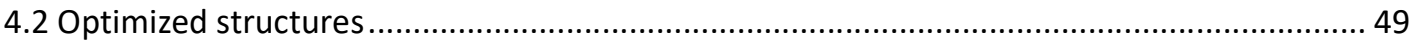

4.3 Identification of the VRI points using the projected frequencies along the IRC ................... 130

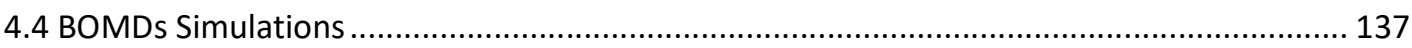




\section{Synthesis}

1.1 General methods

All moisture and air sensitive reactions were conducted in flame-dried glass ware under nitrogen atmosphere. The solutions for the kinetic measurements were degassed using the freeze-pump -thaw method. Commercially available chemicals, if not stated otherwise, were used as purchased without further purification. Solvents were distilled prior to use. Anhydrous solvents were distilled according to standard procedures or bought. Column chromatography was conducted with silica gel 60 by Fluka. For TLC, silica gel $60 \mathrm{~F}_{254}$ coated alumina plates by Macherey-Nagel were used. NMR spectra were measured on Bruker FOURIER 300, Bruker AVANCE 400, Bruker DRX 500 and Bruker AVANCEIII 600 spectrometers and referenced to the residual solvent peak. Characterization of signals: $s=$ singlet; $d$ $=$ doublet $; t=$ triplet $; d d=$ doublet of doublets $; d d d=$ doublet of doublets of doublets $; d d d d=$ doublet of doublets of doublets of doublets; $d t=$ doublet of triplets ; $d d t=$ doublet of doublets of triplets ; $q=$ quartet $; \mathrm{dq}=$ doublet of quartets $; \mathrm{ddq}=$ doublet of doublets of quartets ; sept $=$ septet $; \mathrm{m}=$ multiplet. Mass spectrometry was conducted on a VG $70 \mathrm{~S}$ for El spectra and on an Agilent 6224 ESI-TOF for ESI mass spectra. Infrared spectra were measured on an Alpha Platinum ATR-IR spectrometer by Bruker. The static photolysis experiments were conducted with a Rayonet RPR-100 photochemical reactor using RPR2537A (UV-C), RPR3000A (UV-B) or RPR3500A (UV-A) lamps. Samples were either prepared in standard quartz cuvettes $(\mathrm{d}=1 \mathrm{~cm})$ or in quartz NMR tubes with Young seal.

\subsection{General procedure for the synthesis of $N$-acetyl hydrazones}

A solution of the ketone $(10 \mathrm{mmol}, 1 \mathrm{eq})$, acetyl hydrazine $(11.5 \mathrm{mmol}, 1.15 \mathrm{eq})$ and acetic acid $(0.02$ $\mathrm{mL} / \mathrm{mmol})$ in ethanol $(1 \mathrm{~mL} / \mathrm{mmol})$ was heated to reflux. After cooling down, the solvent was mostly evaporated and the reaction mixture then heated to $120{ }^{\circ} \mathrm{C}$ for three hours. The obtained crude product was recrystallized from acetone or directly used in the following synthesis. ${ }^{22}$

\section{Norcamphor- $\mathrm{N}$-acetyl hydrazone (11-N)}

Synthesis according to general procedure with heating for 30 minutes. The crude product was used without further purification.

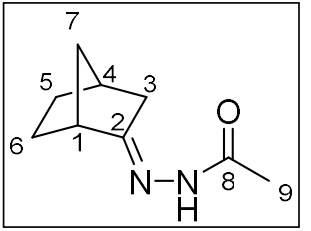

${ }^{1} \mathrm{H}-\mathrm{NMR}\left(\mathrm{CDCl}_{3}, 400 \mathrm{MHz}\right): \delta[\mathrm{ppm}]=8.95(\mathrm{~s}, 0.95 \mathrm{H}, \mathrm{N}-\mathrm{H}), 2.73-2.86(\mathrm{~m}, 1 \mathrm{H}, \mathrm{H}-$ 4), 2.51-2.63 (m, $1 \mathrm{H}, \mathrm{H}-1), 1.59-2.26(\mathrm{~m}+\mathrm{s}(2.20,3 \mathrm{H}, \mathrm{H}-9)+\mathrm{s}(2.19), 11.5 \mathrm{H})$, 1.19-1.54 (m, 5.5H). ${ }^{13} \mathrm{C}-\mathrm{NMR}\left(\mathrm{CDCl}_{3}, 100 \mathrm{MHz}\right): \delta[\mathrm{ppm}]=173.8(\mathrm{C}=\mathrm{O}, \mathrm{C}-8)$, 164.1 (C-2), 44.4 (C-7), 38.8 (C-1), 35.9 (C-4), 35.4 (C-3), 27.7 (C-6), 27.0 (C-5), 20.4 (C-9).

\section{Camphor- $\mathrm{N}$-acetyl hydrazone (11-B)}

Synthesis according to general procedure with heating for $5 \mathrm{~h}$ at $90{ }^{\circ} \mathrm{C}$ in a pressure tube. The crude

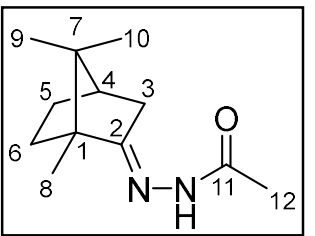
product was recrystallized from acetone to yield $9.5 \mathrm{~g}(46 \mathrm{mmol}, 77 \%)$ of a colorless crystalline solid.

${ }^{1} \mathrm{H}-\mathrm{NMR}\left(\mathrm{CDCl}_{3}, 400 \mathrm{MHz}\right): \delta[\mathrm{ppm}]=9.28(\mathrm{~s}, 1 \mathrm{H}, \mathrm{N}-\mathrm{H}), 8.36(\mathrm{~s}, 1 \mathrm{H}, \mathrm{N}-\mathrm{H}), 2.20-$ $2.40(\mathrm{~m}+\mathrm{s}(2.25,3 \mathrm{H}, \mathrm{H} 12), 4.69 \mathrm{H}), 1.95-2.11(\mathrm{~m}+\mathrm{s}(2.04), 5.02 \mathrm{H}), 1.64-1.95$ $(\mathrm{m}, 5.90 \mathrm{H}), 1.33-1.49(\mathrm{~m}, 1.19 \mathrm{H}), 1.15-1.28(\mathrm{~m}, 1.35 \mathrm{H}), 0.88-1.12(\mathrm{~m}+\mathrm{s}(0.99$, $\mathrm{H}-8)+\mathrm{s}(0.93, \mathrm{H}-10), 7.95 \mathrm{H}), 0.68-0.85(\mathrm{~m}+\mathrm{s}(0.74, \mathrm{H}-9), 3.85 \mathrm{H}) .{ }^{13} \mathrm{C}-\mathrm{NMR}$ $\left(\mathrm{CDCl}_{3}, 100 \mathrm{MHz}\right): \delta[\mathrm{ppm}]=173.5(\mathrm{C}-2), 167.6(\mathrm{C}=0, \mathrm{C}-11), 52.7$ (C-1), 48.0 (C7), 44.2 (C-4), 33.4 (C-3), 32.7 (C-6), 27.4 (C-5), 20.8 (C-12), 19.6 (C-8), 18.8 (C-10), 11.2 (C-9).

\section{Fenchyl- $\mathrm{N}$-acetyl hydrazone (11-F)}

Synthesis according to general procedure with heating for $16 \mathrm{~h}$ at $90^{\circ} \mathrm{C}$ in a pressure tube. Additionally, to heating to $120^{\circ} \mathrm{C}$, the crude mixture was heated in vacuum to remove an excess of fenchone. The

\footnotetext{
${ }^{22}$ Synthesis according to Bekhazi, M.; Risbood, P. A.; Warkentin, J. J. Am. Chem. Soc. 1983, 105, 5675-5679.
} 
crude product was recrystallized from acetone to yield $9.12 \mathrm{~g}(59.9 \mathrm{mmol}, 58 \%)$ of a colorless crystalline solid.

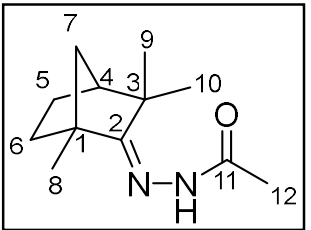

${ }^{1} \mathrm{H}-\mathrm{NMR}\left(\mathrm{CDCl}_{3}, 300 \mathrm{MHz}\right): \delta[\mathrm{ppm}]=8.83(\mathrm{~s}, 1.2 \mathrm{H}, \mathrm{N}-\mathrm{H}), 8.30(\mathrm{~s}, 0.8 \mathrm{H}, \mathrm{N}-\mathrm{H}), 2.22$ (s, 3H, H-12), $1.88(\mathrm{~m}, 1 \mathrm{H}, \mathrm{H}-4), 1.74$ („ „dt“, $3 \mathrm{~J}=6.3,2.8 \mathrm{~Hz}, 1 \mathrm{H}, \mathrm{H}-5 \mathrm{x}), 1.69-1.51$ (m, 3H, H-6x, H-7syn, H-5n), $1.45-1.35$ (m, 2H, H-6n, H-7anti), 1.31 (s, 3H, H-9), 1.23 (s, 3H, H-8), $1.20(\mathrm{~s}, 3 \mathrm{H}, \mathrm{H}-10) .{ }^{13} \mathrm{C}-\mathrm{NMR}\left(\mathrm{CDCl}_{3}, 75 \mathrm{MHz}\right): \delta[\mathrm{ppm}]=173.4$ (C-11), 167.6 (C-2), 51.4 (C-1), 50.0 (C-4), 42.74 (C-3/C-7), 42.70 (C-3/C-7), 34.4 (C-6), 25.2 (C-5), 23.5 (C-9), 23.4 (C-10), 20.8 (C-12), 17.2 (C-8).

1.3 General procedure for the synthesis of 2-methoxy-2-methyl- $\Delta^{3}$-1,3,4-oxadiazolines The synthesis was conducted according to a procedure published by Warkentin et al. [LIT] Lead tetraacetate $(4.44 \mathrm{~g}, 10 \mathrm{mmol}, 1 \mathrm{eq})$ was suspended in dry methanol $(10 \mathrm{~mL})$ and cooled down to $0{ }^{\circ} \mathrm{C}$. Then the $\mathrm{N}$-acetyl hydrazone $(10 \mathrm{mmol}, 1 \mathrm{eq})$ was added and stirred until the yellow color of the solution disappeared $(1-2 \mathrm{~h})$. Potassium hydroxide $(2 \mathrm{~g}, 36 \mathrm{mmol}, 3.6 \mathrm{eq})$ was added and the reaction mixture stirred for $2 \mathrm{~h}$ at $0{ }^{\circ} \mathrm{C}$. The solvent was evaporated and the precipitate extracted with dichloromethane and water. The organic phase was dried over magnesium sulfate and the solvent evaporated in vacuum. The crude mixture was purified by column chromatography, using $\mathrm{PE} / \mathrm{Et}_{2} \mathrm{O}$ mixtures as eluent.

\section{Norbornyl- $\Delta^{3}-1,3,4-$ oxadiazoline (1-N)}

The synthesis was carried out according to the general procedure. The purification was carried out with $\mathrm{PE} / \mathrm{Et}_{2} \mathrm{O} 9: 1 \mathrm{v} / \mathrm{v}$ as solvent mixture to yield $1.5 \mathrm{~g}(7.6 \mathrm{mmol}, 34 \%)$ of a colorless liquid. The
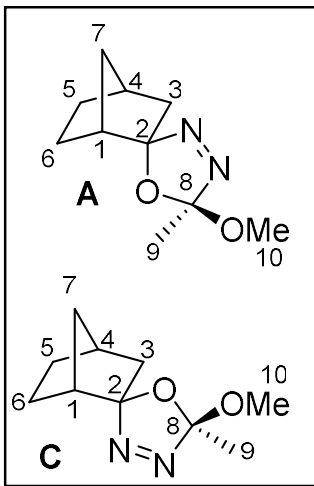
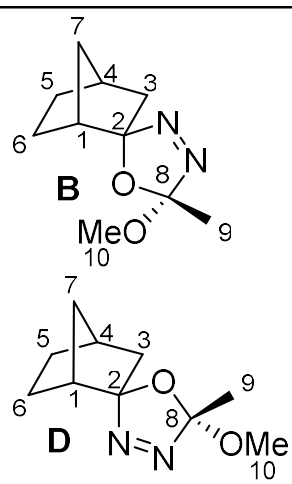
oxadiazoline was isolated as a mixture of four diastereomers with the ratio of $1.0: 1.3: 1.2: 1.2$. ${ }^{1} \mathrm{H}-\mathrm{NMR}\left(\mathrm{CDCl}_{3}, 300 \mathrm{MHz}\right): \delta[\mathrm{ppm}]=3.13(\mathrm{~s}, 0.64 \mathrm{H} / 3 \mathrm{H}$, $\left.\mathrm{OCH}_{3}, \mathrm{~A}\right), 3.05\left(\mathrm{~s}, 0.80 \mathrm{H} / 3 \mathrm{H},-\mathrm{OCH}_{3}, \mathrm{~B}\right), 2.99(\mathrm{~s}, 0.79 \mathrm{H} / 3 \mathrm{H}$, $\left.\mathrm{OCH}_{3}, \mathrm{C}\right), 2.95\left(\mathrm{~s}, 0.75 \mathrm{H} / 3 \mathrm{H},-\mathrm{OCH}_{3}, \mathrm{D}\right), 2.44-2.53(\mathrm{~m}, 1 \mathrm{H}$, $A+B+C+D, H-4), 2.18-2.36(m, 1.53 \mathrm{H}), 2.06-2.18(m, 0.26 \mathrm{H})$, $1.82-2.06(\mathrm{~m}, 1.65 \mathrm{H}), 1.40-1.82(\mathrm{~m}, 8.55 \mathrm{H}$, overlay with $1.62\left(\mathrm{~s},-\mathrm{CH}_{3}\right), 1.58\left(\mathrm{~s},-\mathrm{CH}_{3}\right)$ und $\left.1.55\left(\mathrm{~s}, 2 \mathrm{x}-\mathrm{CH}_{3}\right)\right), 1.37(\mathrm{dd}$, $\left.0.37 \mathrm{H}, 2 J_{\mathrm{H}, \mathrm{H}}=13.3 \mathrm{~Hz}, 3 \mathrm{JH}_{\mathrm{H}, \mathrm{H}}=3.8 \mathrm{~Hz}, \mathrm{H}-7_{\text {syn }}\right), 1.23(\mathrm{dd}, 0.38 \mathrm{H}$, $2 \mathrm{JH}_{\mathrm{H}, \mathrm{H}}=13.1 \mathrm{~Hz}, 3 \mathrm{~J}_{\mathrm{H}, \mathrm{H}}=3.8 \mathrm{~Hz}, \mathrm{H}-7$ anti). ${ }^{13} \mathrm{C}-\mathrm{NMR}\left(\mathrm{CDCl}_{3}, 125\right.$ $\mathrm{MHz}): \delta[\mathrm{ppm}]=133.1$ (CquartA, C-8), 132.7 (CquartB, C-8), 132.6 (Cquartc, C-8), 132.3 (CquartD, C-8), 127.5 (CquartA, C-2),

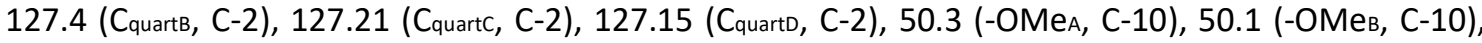
49.97 (-OMec, C-10), 49.95 (-OMed, C-10), 48.3 (C1), 46.8 (C1), 46.2 (C1), 45.2 (C1), 43.3 (C3), 42.9 (C3), 40.5 (C-7), 40.0 (C-7), 39.0 (C-7), 38.8 (C-7), 38.2 (C-3), 38.1 (C-3), 37.0 (C-4), 36.7 (C-4), 36.4 (C-4), 36.3 (C-4), 28.8 (C-5), 28.6 (C-5), 28.3 (C-5), 28.2 (C-5), 23.4 (C-9), 23.2 (C-9), 23.1 (C-6), 23.04 (C-6), 23.02 (C-9), 22.92 (C-9), 22.7 (C-6), 22.5 (C-6).

\section{Solvolytic isomer separation of norbornyl- $\Delta^{3}-1,3,4-0 x a d i a z o l i n e ~(e n d o-1-N)$}

1-N (2.44 g, $12.4 \mathrm{mmol}, 1 \mathrm{eq})$ was solvated in non-anhydrous acetonitrile $(10 \mathrm{~mL}, 0.8 \mathrm{~mL} / \mathrm{mmol})$ and trifluoro acetic acid $(0.8 \mathrm{~mL}, 10.5 \mathrm{mmol}, 0.8 \mathrm{eq})$ added. The reaction mixture was stirred for $2.25 \mathrm{~h}$ and acid and solvent were removed via distillation. The residue was extracted with dichloromethane and water. Purification via column chromatography with $\mathrm{PE} / \mathrm{Et}_{2} \mathrm{O} 9: 1 \mathrm{v} / \mathrm{v}$ as eluent yielded $0.771 \mathrm{~g}$ (3.93 mmol, $32 \%$ ) of a colorless liquid. 


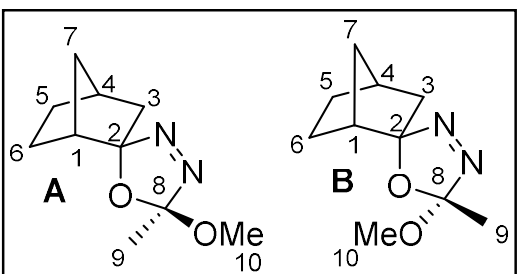

${ }^{1} \mathrm{H}-\mathrm{NMR}\left(\mathrm{CDCl}_{3}, 300 \mathrm{MHz}\right): \delta[\mathrm{ppm}]=3.05\left(\mathrm{~s}, 1.4 \mathrm{H} / 3 \mathrm{H},-\mathrm{OCH}_{3}, \mathrm{~A}\right)$, $2.99\left(\mathrm{~s}, 1.6 \mathrm{H} / 3 \mathrm{H},-\mathrm{OCH}_{3}, \mathrm{C}\right), 2.46-2.54(\mathrm{~m}, 1 \mathrm{H}, \mathrm{H}-4), 2.19-2.37(\mathrm{~m}$, $2 \mathrm{H}), 1.83-2.06(\mathrm{~m}, 2 \mathrm{H}), 1.63-1.81(\mathrm{~m}, 1 \mathrm{H}), 1.42-1.62(\mathrm{~m}, 6 \mathrm{H}$, overlay with $1.58\left(\mathrm{~s},-\mathrm{CH}_{3}\right)$ und $\left.1.55\left(\mathrm{~s},-\mathrm{CH}_{3}\right)\right), 1.37\left(\mathrm{dd}, 2 \mathrm{JH}_{\mathrm{H}, \mathrm{H}}=13.3\right.$ $\mathrm{Hz}, 3 / \mathrm{H}, \mathrm{H}=3.8 \mathrm{~Hz}), 1.23(\mathrm{dd}, 2 \mathrm{JH}, \mathrm{H}=13.1 \mathrm{~Hz}, 3 / \mathrm{H}, \mathrm{H}=3.8 \mathrm{~Hz}) .{ }^{13} \mathrm{C}-\mathrm{NMR}$ $\left(\mathrm{CDCl}_{3}, 75 \mathrm{MHz}\right): \delta[\mathrm{ppm}]=133.2$ (CquartA, C-8), 132.7 (Cquartc, C-8), 127.6 (Cquart',$C-2), 127.4$ (Cquart', C-2), 50.2 (-OMe, C-10), 50.1 (OMe, C-10), 46.3 (C1), 45.3 (C1), 40.6 (C-7), 40.1 (C-7), 38.34 (C-3), 38.25 (C-3), 37.1 (C-4), 36.8 (C-4), 28.9 (C-5), 28.7 (C-5), 23.1 (C-9), 23.0 (C-6), 22.8 (C-9), 22.6 (C-6).

\section{Bornyl- $\Delta^{3}-1,3,4-o x a d i a z o l i n e ~(1-B)$}

The synthesis was carried out according to the general procedure. The purification was carried out with a gradient ranging from $\mathrm{PE} / \mathrm{Et}_{2} \mathrm{O} 20: 1 \mathrm{v} / \mathrm{v}$ to $13: 1 \mathrm{v} / \mathrm{v}$ to yield $5.94 \mathrm{~g}(24.9 \mathrm{mmol}, 46 \%)$ of a colorless oily liquid. The oxadiazoline was isolated as a mixture of four diastereomers with the ratio of

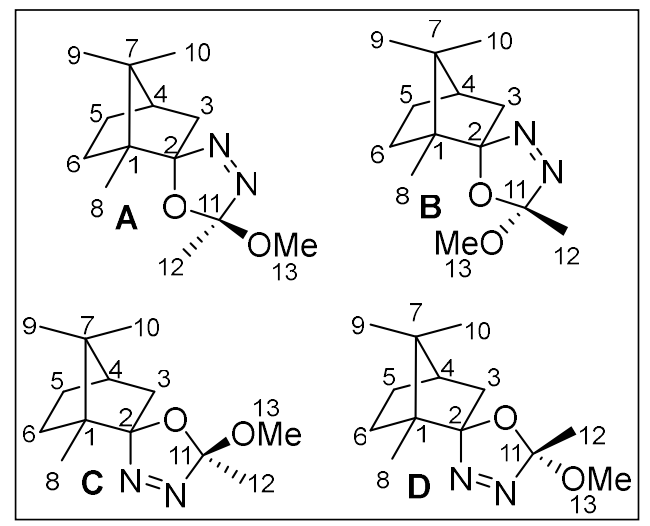

$1.0: 2.1: 3.8: 2.7$

${ }^{1} \mathrm{H}-\mathrm{NMR}\left(\mathrm{CDCl}_{3}, 300 \mathrm{MHz}\right): \delta[\mathrm{ppm}]=3.28(\mathrm{~s}, 0.30 \mathrm{H} / 3 \mathrm{H}$, $\left.\mathrm{OCH}_{3}, \mathrm{~A}\right), 3.26\left(\mathrm{~s}, 0.64 \mathrm{H} / 3 \mathrm{H},-\mathrm{OCH}_{3}, \mathrm{~B}\right), 2.99(\mathrm{~s}, 1.14 \mathrm{H} / 3 \mathrm{H}$, $\left.-\mathrm{OCH}_{3}, \mathrm{C}\right), 2.94\left(\mathrm{~s}, 0.82 \mathrm{H} / 3 \mathrm{H},-\mathrm{OCH}_{3}, \mathrm{D}\right), 2.59-2.66(\mathrm{~m}$, $0.14 \mathrm{H}, \mathrm{H}-3$ aq $(\mathrm{A})), 2.48\left(\mathrm{ddd}, 0.42 \mathrm{H}, 2 \mathrm{JH}_{\mathrm{H}, \mathrm{H}}=13.7 \mathrm{~Hz}, 3 / \mathrm{H}, \mathrm{H}=\right.$ $4.5 \mathrm{~Hz}, 3.1 \mathrm{~Hz}, \mathrm{H}-3 \mathrm{aq}(\mathrm{C})), 2.34-2.40(\mathrm{~m}, 0.32 \mathrm{H}, \mathrm{H}-3 \mathrm{aq}(\mathrm{D}))$, 2.24 (ddd, $0.25 \mathrm{H}, 2 J_{\mathrm{H}, \mathrm{H}}=13.4 \mathrm{~Hz}, 3 J_{\mathrm{H}, \mathrm{H}}=4.4 \mathrm{~Hz}, 3.2 \mathrm{~Hz}, \mathrm{H}-$ 3aq (B)), 1.95-2.07 (m, 0.94H), 1.79-1.95 (m, 2.36H), 1.68$1.72\left(\mathrm{~m}, 1.97 \mathrm{H},\left(\mathrm{s}+\mathrm{s}, 2 \mathrm{x}-\mathrm{CH}_{3}\right)\right), 1.34-1.66(\mathrm{~m}, 5.94 \mathrm{H}$, overlay with $1.54\left(\mathrm{~s},-\mathrm{CH}_{3}\right), 1.49\left(\mathrm{~s},-\mathrm{CH}_{3}\right), 1.47\left(\mathrm{~s},-\mathrm{CH}_{3}\right)$, $1.43\left(\mathrm{~s},-\mathrm{CH}_{3}\right)$ und $\left.1.41\left(\mathrm{~s},-\mathrm{CH}_{3}\right)\right), 1.06\left(\mathrm{~s}, 0.3 \mathrm{H},-\mathrm{CH}_{3},(\mathrm{~A})\right)$, $1.04\left(\mathrm{~s}, 0.8 \mathrm{H},-\mathrm{CH}_{3},(\mathrm{D})\right), 0.96-1.00(\mathrm{~m}, 1.8 \mathrm{H}$, overlay of $0.98\left(\mathrm{~s},-\mathrm{CH}_{3}\right)$ und $\left.0.99\left(\mathrm{~s},-\mathrm{CH}_{3}\right)\right), 0.90-0.93(\mathrm{~m}, 1.12 \mathrm{H},(\mathrm{s}+$ $\left.\left.\mathrm{s}, 2 \mathrm{x}-\mathrm{CH}_{3}\right)\right), 0.73-0.77\left(\mathrm{~m}, 0.94 \mathrm{H},\left(\mathrm{s}+\mathrm{s}, 2 \mathrm{x}-\mathrm{CH}_{3}\right), \mathrm{A}+\mathrm{B}\right), 0.61(\mathrm{~s}, 0.8 \mathrm{H}, \mathrm{D}), 0.57(\mathrm{~s}, 1.16 \mathrm{H}, \mathrm{C}) .{ }^{13} \mathrm{C}-\mathrm{NMR}$ $\left(\mathrm{CDCl}_{3}, 75 \mathrm{MHz}, \mathrm{DEPT}\right): \delta[\mathrm{ppm}]=129.2,128.4,125.6,125.4,55.1,54.7,51.0,50.3,50.1,49.6,45.6$, $45.3,41.0,40.8,30.9,30.4,27.4,23.2,21.0,20.9,20.3,19.7 .{ }^{13} \mathrm{C}-\mathrm{NMR}\left(125 \mathrm{MHz}, \mathrm{CDCl}_{3}, 2{ }^{\circ} \mathrm{C}\right): \delta[\mathrm{ppm}]$

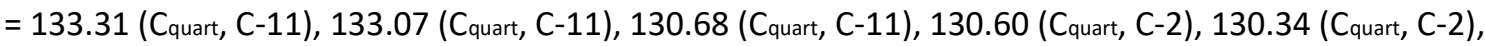

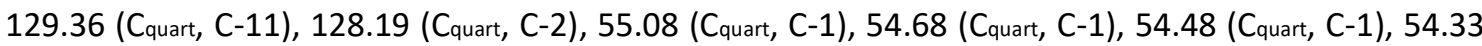
(Cquart, C-1), 51.18 (-OMe, C-13), 50.94 (-OMe, C-13), 50.25 (-OMe, C-13), 50.09 (-OMe, C-13), 49.61 (Cquart, C-7), 49.57 ( Cquart, $_{\text {C-7) }}, 49.54$ (Cquart, C-7), 49.47 (Cquart, C-7), 45.53 (C-4), 45.41 (C-4), 45.28 (C-4), 45.25 (C-4), 42.92 (C-5), 42.77 (C-5), 40.98 (C-3), 40.72 (C-3), 30.84 (C-3), 30.79 (C-3), 30.37 (C-5), 30.29 (C-5), 27.39 (C-6), 27.36 (C-6), 27.30 (C-6), 27.19 (C-6), 23.20 (C-12), 23.19 (C-12), 22.85 (C-12), 22.46 (C-12), 20.98 (C-10), 20.90 (C-9), 20.75 (C-10), 20.69 (C-9), 20.29 (C-10), 20.26 (C-10), 19.64 (C-9), 19.63 (C-9), 11.23 (C-8), 10.79 (C-8), 10.22 (C-8), 10.08 (C-8).

\section{Fenchyl- $\Delta^{3}-1,3,4$-oxadiazoline (1-F)}

The synthesis was carried out according to the general procedure. The purification was carried out with a gradient ranging from $\mathrm{PE} / \mathrm{Et}_{2} \mathrm{O} 30: 1 \mathrm{v} / \mathrm{v}$ to $10: 1 \mathrm{v} / \mathrm{v}$ to yield $0.631 \mathrm{~g}(2.65 \mathrm{mmol}, 11 \%)$ of a colorless oily liquid. The oxadiazoline was isolated as a mixture of four diastereomers with the ratio of $1.0: 5.8: 5.4: 2.2$. 


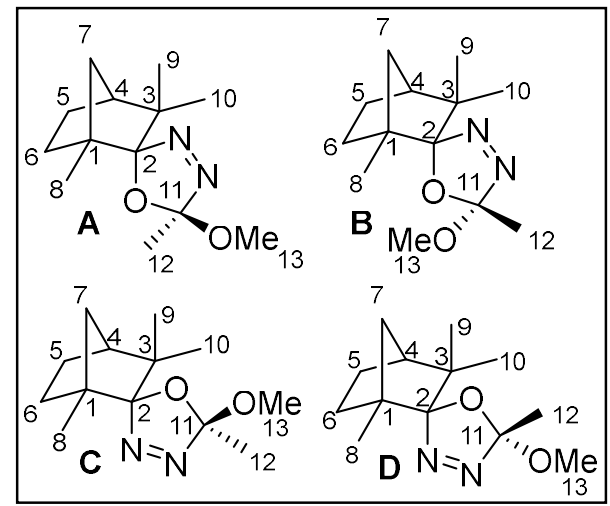

${ }^{1} \mathrm{H}-\mathrm{NMR}\left(\mathrm{CDCl}_{3}, 400 \mathrm{MHz}\right): \delta[\mathrm{ppm}]=3.47(\mathrm{~s}, 0.21 \mathrm{H} / 3 \mathrm{H},-$ $\left.\mathrm{OCH}_{3}, \mathrm{~A}\right), 3.44\left(\mathrm{~s}, 1.2 \mathrm{OH} / 3 \mathrm{H},-\mathrm{OCH}_{3}, \mathrm{~B}\right), 3.41(\mathrm{~s}, 1.10 \mathrm{H} / 3 \mathrm{H}$, $\left.\mathrm{OCH}_{3}, \mathrm{C}\right), 3.39\left(\mathrm{~s}, 0.45 \mathrm{H} / 3 \mathrm{H},-\mathrm{OCH}_{3}, \mathrm{D}\right), 2.57\left(\mathrm{dq}, 0.55 \mathrm{H}, 2 \mathrm{JH}_{\mathrm{H}, \mathrm{H}}\right.$ $=10.5 \mathrm{~Hz}, 3 / \mathrm{H}, \mathrm{H}=2.2 \mathrm{~Hz}, \mathrm{H}-7_{\text {syn }}$ ), 2.47 (dddd, $0.5 \mathrm{H}, 2 / \mathrm{H}, \mathrm{H}=13.0$ $\left.\mathrm{Hz}, 3 \mathrm{~J}_{\mathrm{H}, \mathrm{H}}=9.1 \mathrm{~Hz}, 5.7 \mathrm{~Hz}, 2.2 \mathrm{~Hz}, \mathrm{H}-7_{\text {syn }}\right), 2.02$ (ddt, $0.51 \mathrm{H}, 2 \mathrm{JH}, \mathrm{H}$ $\left.=12.4 \mathrm{~Hz}, 3 \mathrm{~J}_{\mathrm{H}, \mathrm{H}}=9.1 \mathrm{~Hz}, 3.3 \mathrm{~Hz}, \mathrm{H}-4\right), 1.86-1.94(\mathrm{~m}, 1 \mathrm{H}, \mathrm{H}-4)$, 1.75-1.86 (m, 1.5H, H-5aq $+\mathrm{H}-6 \mathrm{aq}), 1.74\left(\mathrm{~s}, 1.34 \mathrm{H},-\mathrm{CH}_{3}, \mathrm{H}-12\right)$, 1.73 (s, 1.10, $\left.-\mathrm{CH}_{3}, \mathrm{H}-12\right)$ ) 1.51-1.66 (m, 2H, overlay with $\left.1.63\left(\mathrm{~s},-\mathrm{CH}_{3}\right), 1.60\left(\mathrm{~s},-\mathrm{CH}_{3}\right), \mathrm{H}-5 \mathrm{ax}+\mathrm{H}-6 \mathrm{ax}+\mathrm{H} 12\right), 1.36-1.46(\mathrm{~m}$, $\left.1.1 \mathrm{H}, \mathrm{H}-7_{\mathrm{anti}}\right), 1.18-1.29\left(\mathrm{~m}, 1.5 \mathrm{H}, \mathrm{H}-5_{\mathrm{ax}}+\mathrm{H}-6 \mathrm{ax}\right), 1.01$ (s, $\left.1.47 \mathrm{H},-\mathrm{CH}_{3}, \mathrm{H}-9\right), 0.97\left(\mathrm{~s}, 0.48 \mathrm{H},-\mathrm{CH}_{3}, \mathrm{H}-10\right), 0.96(\mathrm{~s}, 1.16 \mathrm{H}$, $\left.-\mathrm{CH}_{3}, \mathrm{H}-10\right), 0.90\left(\mathrm{~s}, 1.51 \mathrm{H},-\mathrm{CH}_{3}, \mathrm{H}-10\right), 0.84-0.87(\mathrm{~m}(\mathrm{~s}+\mathrm{s})$, $\left.1.73 \mathrm{H}, 2 \mathrm{x}-\mathrm{CH}_{3}, \mathrm{H}-9\right), 0.81\left(\mathrm{~s}, 0.69 \mathrm{H},-\mathrm{CH}_{3}, \mathrm{H}-8\right), 0.74\left(\mathrm{~s}, 1.26 \mathrm{H},-\mathrm{CH}_{3}, \mathrm{H}-8\right), 0.71\left(\mathrm{~s}, 1.16 \mathrm{H},-\mathrm{CH}_{3}, \mathrm{H}-8\right) .{ }^{13} \mathrm{C}-$ NMR $\left(\mathrm{CDCl}_{3}, 125 \mathrm{MHz}\right): \delta$ [ppm] = 132.10 (Cquart, $\left.\mathrm{C}-2 / 11\right), 131.94$ (Cquart, $\left.\mathrm{C}-2 / 11\right), 131.75$ (Cquart, $\left.\mathrm{C}-2 / 11\right)$, 131.43 (Cquart, C-2/11), 131.40 (Cquart, C-2/11), 131.15 (Cquart, C-2/11), 130.5 (Cquart, C-2/11), 130.2 (Cquart, C-2/11), 53.5 (C quart, C-1), 52.9 (Cquart, C-1), 52.6 (Cquart, C-1), 51.5 (-OMe, C-13), 51.31 (-OMe, C-13), 51.27 (-OMe, C-13), 51.17 (-OMe, C-13), 49.2 (C-4), 49.0 (C-4), 48.6 (C-4), 48.5 (C-4), 45.1 (C-3), 44.9 (C-3), 43.3 (C-6), 43.0 (C-6), 41.9 (C-7), 41.5 (C-7), 31.5 (C-7, B), 31.4 (C-7, A), 29.8 (C-6, D), 29.6 (C-6, C), 26.4, 26.1 (C-5), 26.02, 25.97 (C-5), 25.95 (C-5), 25.90 (C-5), 25.69 (C-9), 25.67 (C-9), 23.2 (C-10, D), 22.9 (C-10, C), 22.7 (C-10, A), 22.0 (C-9, B), 21.2 (C-12, D), 20.5 (C-12, A), 19.7 (C-12, C), 19.4 (C-12, B), $16.3(C-8, A), 16.0(C-8, B), 15.7(C-8, D), 15.4(C-8, C)$.

\subsection{Syntheses of reference compounds}

\section{Synthesis of exo-2-(1,1,1,3,3,3-hexafluoroisopropyl)-oxynorbornane (6-N)}

Norbornene ( $2.0 \mathrm{~g}, 21 \mathrm{mmol}, 1 \mathrm{eq}), 1,1,1,3,3,3$-hexafluoro-2-propanol (HFIP, $4.4 \mathrm{~mL}, 42 \mathrm{mmol}, 2 \mathrm{eq})$ and copper(II) triflate ( $252 \mathrm{mg}, 0.7 \mathrm{mmol}, 0.03 \mathrm{eq}$ ) were dissolved in dioxane ( $12 \mathrm{~mL}, 0.55 \mathrm{~mL} / \mathrm{mmol}$ ) and heated to $80^{\circ} \mathrm{C}$ in a pressure flask for 4 hours. The reaction was then stirred an additional $19 \mathrm{~h}$ at room temperature. The reaction mixture was extracted with diethyl ether and water. The purification

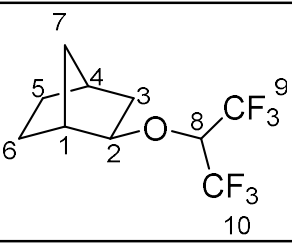
via column chromatography ( $\mathrm{PE} / \mathrm{Et}_{2} \mathrm{O} 20: 1 \mathrm{v} / \mathrm{v}$ ) yielded $2.54 \mathrm{~g}$ (10.1 mmol, $48 \%$ ) of a colorless oil.

${ }^{1} \mathrm{H}-\mathrm{NMR}\left(\mathrm{CDCl}_{3}, 300 \mathrm{MHz}\right): \delta[\mathrm{ppm}]=4.10\left(\mathrm{sept}, 3 \mathrm{~J}_{\mathrm{H}, \mathrm{F}}=6.0 \mathrm{~Hz}, 1 \mathrm{H}, \mathrm{H}-8\right), 3.78$ (, d“'(dd), 3/H,H $=7.3 \mathrm{~Hz}, 1 \mathrm{H}, \mathrm{H}-2), 2.37$ (, d“', 3/H,H $=4.9 \mathrm{~Hz}, 1 \mathrm{H}, \mathrm{H}-1), 2.27-2.33(\mathrm{~m}$, $1 \mathrm{H}, \mathrm{H}-4), 1.37-1.71\left(\mathrm{~m}, 5 \mathrm{H}, \mathrm{H}-3 \mathrm{aq}+\mathrm{H}-3 \mathrm{ax}+\mathrm{H}-5_{\mathrm{aq}}+\mathrm{H}-6_{\mathrm{aq}}+\mathrm{H}-7\right.$ syn), 1.16 (ddq, $2 \mathrm{JH}_{\mathrm{H}, \mathrm{H}}=9.9$ $\mathrm{Hz}, 3 / \mathrm{H}, \mathrm{H}=2.8 \mathrm{~Hz}, 4 \mathrm{JH}_{\mathrm{H}, \mathrm{H}}=1.5 \mathrm{~Hz}, 1 \mathrm{H}, \mathrm{H}-7$ anti) $), 0.90-1.10$ (m, 2H, H-5ax+H-6ax). ${ }^{13} \mathrm{C}-$ NMR $\left(\mathrm{CDCl}_{3}, 75 \mathrm{MHz}\right): \delta[\mathrm{ppm}]=121.6($,q“, C-9+C-10), 87.2 (C-2), 74.3 (sept, C8), 40.9 (C-1), 39.2 (C-3), 35.4 (C-4), 34.6 (C-7), 28.4 (C-5), 24.1 (C-6). ${ }^{19} \mathrm{~F}-\mathrm{NMR}$ (CDCl, $565 \mathrm{MHz}$ ): $\delta$ $[\mathrm{ppm}]=-74.13\left(\mathrm{qd}, \mathrm{J}_{\mathrm{H}, \mathrm{F}}=9.1,6.2\right),-74.32\left(\mathrm{qd}, 3 \mathrm{~J}_{\mathrm{H}, \mathrm{F}}=9.2,6.3\right)$.

\section{Synthesis of 2-Isopropyl-2',4',6'-trimethoxy-5-methyl-1,2,3,4-tetrahydro-1,1'-biphenyl (10)}

Fenchyl- $\Delta^{3}$-1,3,4-oxadiazoline (1-F) (106 mg, $0.44 \mathrm{mmol}, 1 \mathrm{eq}$ ) and 1,3,5-trimethoxybenzene (143 mg, $0.85 \mathrm{mmol}, 1.9 \mathrm{eq})$ were dissolved in 1,1,1,3,3,3-hexafluoro isopropanol $(6 \mathrm{~mL}, 13.6 \mathrm{~mL} / \mathrm{mmol})$. The colorless solution was photolyzed in three batches of $2 \mathrm{~mL}$ for 2 hours in a photo reactor with UV-B lamps in quartz cuvettes. The solution became a pale pink. The solvent was evaporated and the combined batches purified via column chromatography $\left(\mathrm{PE} / \mathrm{Et}_{2} \mathrm{O} 9: 1 \mathrm{v} / \mathrm{v}\right)$ to yield $33.2 \mathrm{mg}(0.11 \mathrm{mmol}$, $25 \%)$ of a colorless oil.

When using the Bornyl- $\Delta^{3}-1,3,4$-oxadiazoline (1-B), the same product was isolated with lower conversion. 


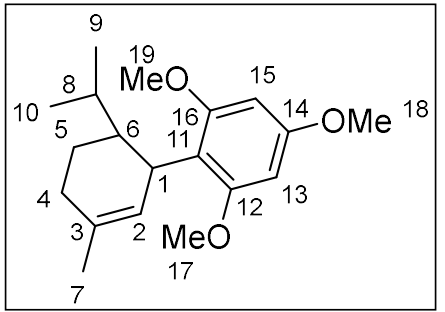

(C quart, C-11), 91.5 (C-13+C-15), 56.0 (-OMe, C-17+C19), 55.2 (-OMe, C-18), 42.2 (C-6), 35.6 (C-1), 31.0 (C-4), 28.4 (C-8), 23.4 (C-7), 23.1 (C-5), 21.6 (C-10), 16.2 (C-9).

\section{Synthesis of exo-2-Norbornyl-(2,2,2-trifluoro) acetate (5-N)}

Norbornene $(2.0 \mathrm{~g}, 21 \mathrm{mmol}, 1 \mathrm{eq}), 2,2,2$-trifluoro acetic acid $(3.1 \mathrm{~mL}, 4.56 \mathrm{~g}, 40 \mathrm{mmol}, 2.0 \mathrm{eq})$ and copper(II) triflate $(256 \mathrm{mg}, 0.70 \mathrm{mmol}, 0.03 \mathrm{eq})$ were dissolved in dioxane $(12 \mathrm{~mL}, 0.55 \mathrm{~mL} / \mathrm{mmol})$ and stirred in a pressure flask for $4 \mathrm{~h}$ at $80^{\circ} \mathrm{C}$ and for $19 \mathrm{~h}$ at room temperature. When the reaction was finished, the mixture was extracted with diethyl ether and water. Purification via column chromatography ( $\left.\mathrm{PE} / \mathrm{Et}_{2} \mathrm{O} 20: 1 \mathrm{v} / \mathrm{v}\right)$ and subsequent distillation yielded $2.26 \mathrm{~g}(10.9 \mathrm{mmol}, 52 \%)$ of a colorless oil.

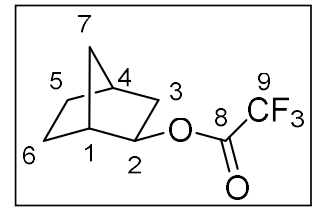

${ }_{1} \mathrm{H}-\mathrm{NMR}\left(\mathrm{CDCl}_{3}, 300 \mathrm{MHz}\right): \delta[\mathrm{ppm}]=4.81\left(, \mathrm{~d}\right.$ “ $\left.(\mathrm{dd}), 3 \mathrm{~J}_{\mathrm{H}, \mathrm{H}}=7.0 \mathrm{~Hz}, 1 \mathrm{H}, \mathrm{H}-2\right)$, $2.36\left(,{ }^{\prime \prime}{ }^{\prime \prime}, 3{ }^{\mathrm{H}, \mathrm{H}}=4.5 \mathrm{~Hz}, 1 \mathrm{H}, \mathrm{H}-1\right), 2.31-2.39(\mathrm{~m}, 1 \mathrm{H}, \mathrm{H}-4), 1.81$ (ddd, $2 \mathrm{JH}_{\mathrm{H}, \mathrm{H}}=13.8$ $\left.\mathrm{Hz}, 3 \mathrm{~J}_{\mathrm{H}, \mathrm{H}}=7.0 \mathrm{~Hz}, 4 \mathrm{JH}_{\mathrm{H}, \mathrm{H}}=2.5 \mathrm{~Hz}, 1 \mathrm{H}, \mathrm{H}-3 \mathrm{aq}\right), 1.41-1.67\left(\mathrm{~m}, 4 \mathrm{H}, \mathrm{H}-3 \mathrm{ax}+\mathrm{H}-5_{\mathrm{aq}}+\mathrm{H}-6_{\mathrm{aq}}+\mathrm{H}-\right.$ 7 syn), $1.24\left(\mathrm{ddq}, 2 \mathrm{JH}_{\mathrm{H}, \mathrm{H}}=10.2 \mathrm{~Hz}, 3 J_{\mathrm{H}, \mathrm{H}}=2.8 \mathrm{~Hz}, 4 \mathrm{~J}_{\mathrm{H}, \mathrm{H}}=1.4 \mathrm{~Hz}, 1 \mathrm{H}, \mathrm{H}-7\right.$ anti), $1.03-1.20$ $(\mathrm{m}, 2 \mathrm{H}, \mathrm{H}-5 \mathrm{ax}+\mathrm{H}-6 \mathrm{ax}) .13 \mathrm{C}-\mathrm{NMR}\left(\mathrm{CDCl}_{3}, 75 \mathrm{MHz}\right): \delta[\mathrm{ppm}]=157.2$ (q, C-8), 114.7 (q, C-9), 82.2 (C-2), 41.5 (C-1), 39.2 (C-3),35.5 (C-4), 35.3 (C-7), 28.0 (C-5), 24.0 (C-6). 19F-NMR (CDCl , $565 \mathrm{MHz}): \delta[\mathrm{ppm}]=-75.60(\mathrm{~m})$. 


\section{Kinetic studies}

\subsection{Photolysis of Norcamphor Precursor 1-N in ACN}

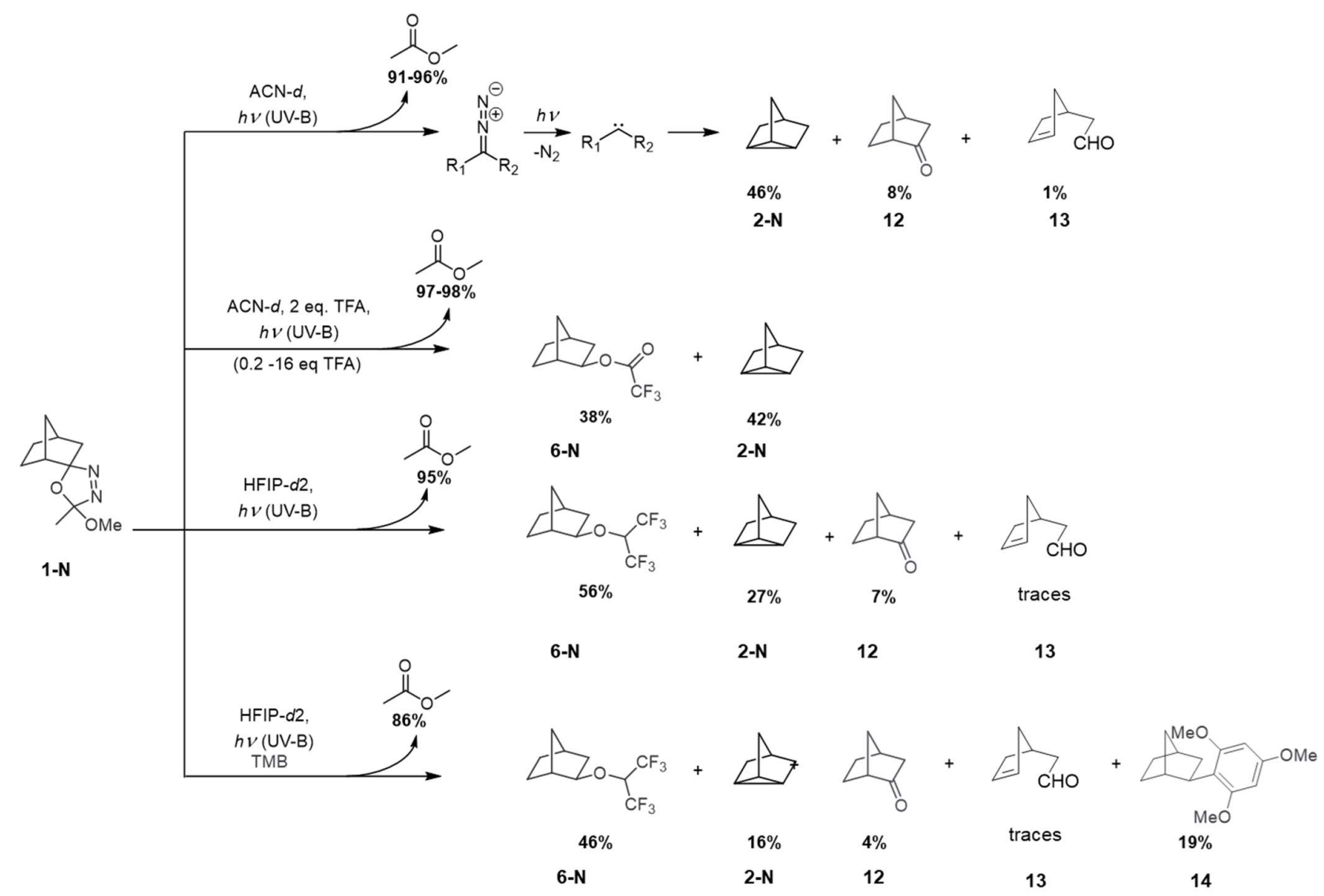

Scheme S4 Overview of products observed and characterized in the UV-B photolysis of 1-N under different conditions. 


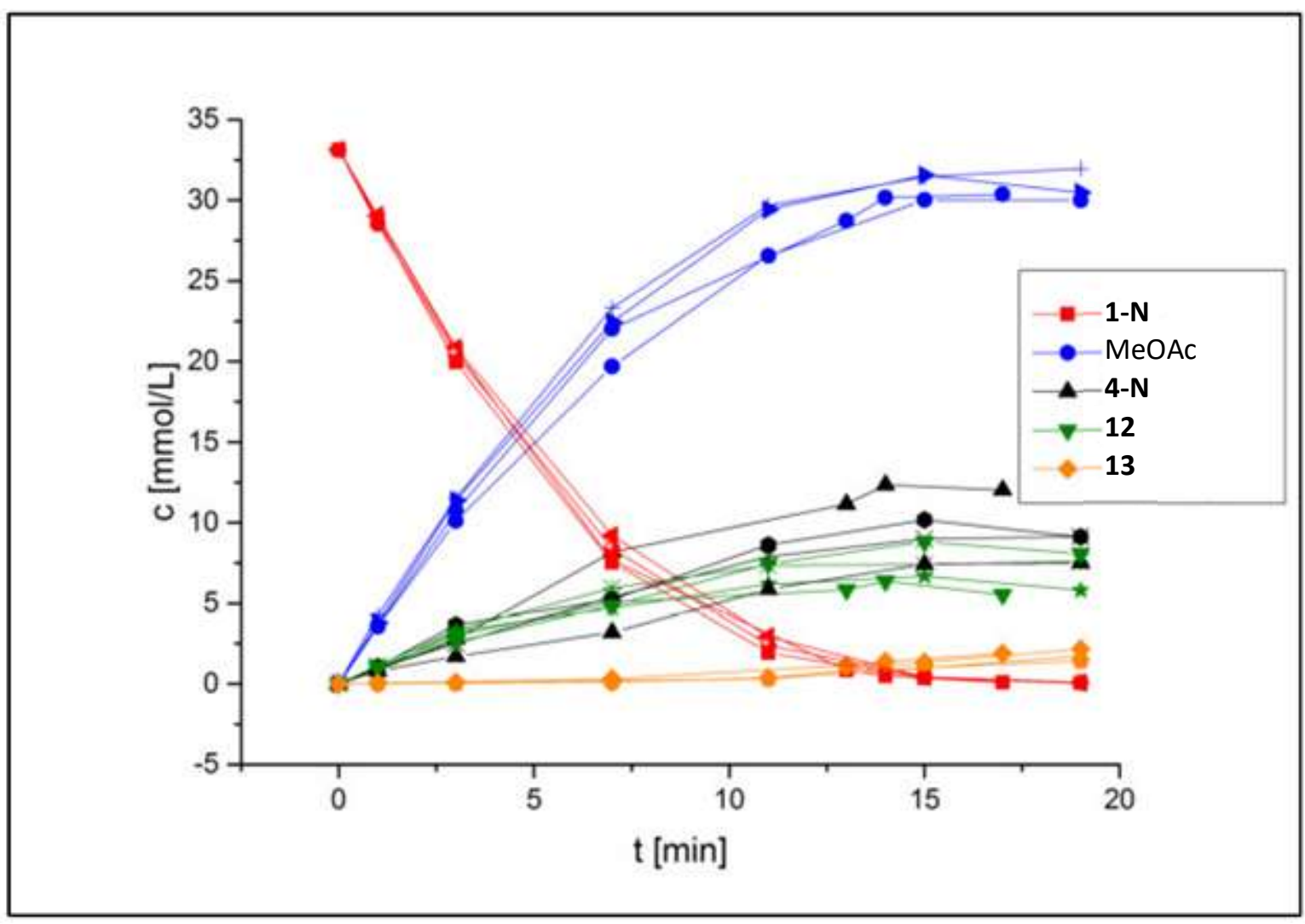

Figure S4: Kinetic trace of the photolysis of $\mathbf{1 - N}$ in $A C N-d_{3}$. The photolysis was carried out in a quartz NMR tube with UV-B light under exclusion of air and rate constants were determined by four independent runs. 
Table S3: Concentrations (c) of the photolysis products determined by ${ }^{1} \mathrm{H}$ NMR in the UVB irradiation of 1-N (quartz NMR tube) in ACN- $d_{3}$ (kinetic traces see Figure S1).

\begin{tabular}{ccccccccc}
\hline & & & & \multicolumn{2}{c}{$c[\mathrm{mmol} / \mathrm{L}]$} & & & \\
$\mathrm{t}$ & $\mathbf{1 - N}$ & $\mathbf{1 - N}$ & $\mathbf{1 - N}$ & $\mathbf{1 - N}$ & $\mathrm{MeOAc}$ & $\mathrm{MeOAc}$ & $\mathrm{MeOAc}$ & MeOAc \\
\hline $\mathrm{min}]$ & run 1 & run 2 & run 3 & run 4 & run 1 & run 2 & run 3 & run 4 \\
\hline 0 & 33.12103 & 33.12103 & 33.12103 & 33.12103 & 0 & 0 & 0 & 0 \\
1 & 28.85504 & 29.05045 & 28.52383 & 28.69937 & 3.59032 & 3.80229 & 4.24612 & 3.54395 \\
3 & 20.72051 & 20.84969 & 20.42243 & 19.95542 & 10.73784 & 11.38039 & 11.51949 & 10.10191 \\
7 & 7.65758 & 9.20433 & 8.37962 & 7.52179 & 22.02548 & 22.44943 & 23.34039 & 19.68383 \\
11 & - & 2.93121 & 2.3814 & 1.93427 & - & 29.42141 & 29.68969 & 26.55313 \\
13 & 0.8479 & - & - & - & 28.74243 & - & - & - \\
14 & 0.49019 & - & - & - & 30.16001 & - & - & - \\
15 & - & 0.4372 & 0.42064 & 0.36102 & - & 31.55109 & 31.45504 & 30.01096 \\
17 & 0.09936 & - & - & - & 30.36205 & - & - & - \\
19 & - & 0.06293 & 0.09274 & 0.05962 & - & 30.47797 & 31.95517 & 30.00434 \\
\hline max. & $0.3 \%$ & $0.2 \%$ & $0.3 \%$ & $0.2 \%$ & $92 \%$ & $92 \%$ & $96 \%$ & $91 \%$ \\
conv. & & & & & & & & \\
\hline
\end{tabular}

Table S4: Concentrations (c) of the photolysis products determined by ${ }^{1} \mathrm{H}$ NMR in the UVB irradiation of 1-N (quartz NMR tube) in ACN- $d_{3}$ (kinetic traces see Figure $\mathrm{S} 1$ ).

\begin{tabular}{ccccccccc}
\hline $\mathrm{t}$ & $\mathbf{4}-\mathbf{N}$ & $\mathbf{4 - N}$ & $\mathbf{4 - N}$ & $\mathbf{4 - N}$ & $\mathbf{1 2}$ & $\mathbf{1 2}$ & $\mathbf{1 2}$ & $\mathbf{1 2}$ \\
{$[\mathrm{min}]$} & run 1 & run 2 & run 3 & run 4 & run 1 & run 2 & run 3 & run 4 \\
\hline 0 & 0 & 0 & 0 & 0 & 0 & 0 & 0 & 0 \\
1 & 0.8479 & 1.05987 & 0.9572 & 0.8214 & 1.0135 & 0.83465 & 1.13274 & 1.02344 \\
3 & 2.79541 & 3.65656 & 2.50726 & 1.70905 & 2.99083 & 3.25911 & 3.32866 & 2.61325 \\
7 & 8.17758 & 5.25962 & $5-39873$ & 3.18955 & 5.06752 & 4.69987 & 5.94191 & 4.84892 \\
11 & - & 8.61478 & 7.89605 & 5.87898 & - & 6.19032 & 7.35287 & 7.45223 \\
13 & 11.15185 & - & - & - & 5.83261 & - & - & - \\
14 & 12.36408 & - & - & - & 6.37911 & - & - & - \\
15 & - & 10.17478 & 8.99567 & 7.43567 & - & 6.68714 & 7.41249 & 8.81351 \\
17 & 12.03618 & - & - & - & 5.52459 & - & - & - \\
\hline
\end{tabular}




\begin{tabular}{ccccccccc}
\hline 19 & - & 9.11491 & 9.13809 & 7.51847 & - & 5.8028 & 7.59134 & 8.07822 \\
$\begin{array}{l}\text { max. } \\
\text { conv. }\end{array}$ & $36 \%$ & $28 \%$ & $28 \%$ & $23 \%$ & $17 \%$ & $18 \%$ & $23 \%$ & $24 \%$ \\
\hline
\end{tabular}

Table S5: Concentrations (c) of the photolysis products determined by ${ }^{1} \mathrm{H}$ NMR in the UVB irradiation of 1-N (quartz NMR tube) in ACN- $\mathrm{d}_{3}$ (kinetic traces see Figure S1).

\begin{tabular}{ccccc}
\hline \multicolumn{5}{c}{$c[\mathrm{mmol} / \mathrm{L}]$} \\
$\mathrm{t}$ & $\mathbf{1 3}$ & $\mathbf{1 3}$ & $\mathbf{1 3}$ & $\mathbf{1 3}$ \\
{$[\mathrm{min}]$} & run 1 & run 2 & run 3 & run 4 \\
\hline 0 & 0 & 0 & 0 & 0 \\
1 & 0.03975 & 0.02981 & 0.01987 & 0.02981 \\
3 & 0.11924 & 0.04968 & 0.04968 & 0.08943 \\
7 & 0.31796 & 0.13911 & 0.18879 & 0.11924 \\
11 & - & 0.3279 & 0.3279 & 0.36764 \\
13 & 1.14268 & - & - & - \\
14 & 1.39108 & - & - & - \\
15 & - & 1.00357 & 0.98369 & 1.3414 \\
17 & 1.89783 & - & - & - \\
19 & - & 1.42089 & 1.73885 & 2.15618 \\
\hline max. & $6 \%$ & $4 \%$ & $5 \%$ & $7 \%$ \\
conv. & & & & \\
\hline
\end{tabular}

Table S6: Rate constants (mono exponential fit) of the different diastereomers (A-D) of 1-N in the photolysis experiment with UV-B light in $A C N-d_{3}$ (quartz NMR tubes) under exclusion of air.

\begin{tabular}{|c|c|c|c|c|c|c|}
\hline & \multicolumn{4}{|c|}{$\mathrm{k}\left[\mathrm{min}^{-1}\right]$} & \multirow{3}{*}{$\begin{array}{c}\mathrm{k} \emptyset \text {,isomers } \\
{\left[\mathrm{min}^{-1}\right]}\end{array}$} & \multirow{3}{*}{$\mathrm{k}_{\emptyset, \text { total }}{ }^{a}\left[\mathrm{~min}^{-1}\right]$} \\
\hline & Isomer A & Isomer B & Isomer C & Isomer D & & \\
\hline & endo-1-N & endo-1-N & endo-1-N & endo-1-N & & \\
\hline \multirow[t]{3}{*}{ run 1} & $0.1679 \pm$ & $0.1810 \pm$ & $0.1646 \pm$ & $0.1561 \pm$ & 0.167 & $0.1628 \pm$ \\
\hline & 0.0198 & 0.0176 & 0.0194 & 0.0156 & $\sigma=0.010$ & 0.0193 \\
\hline & $R^{2}=0.994$ & $R^{2}=0.996$ & $R^{2}=0.995$ & $R^{2}=0.996$ & & $R^{2}=0.995$ \\
\hline \multirow[t]{3}{*}{ run 2} & $0.1691 \pm$ & $0.1750 \pm$ & $0.1658 \pm$ & $0.1599 \pm$ & 0.167 & $0.1581 \pm$ \\
\hline & 0.0202 & 0.0222 & 0.0217 & 0.0183 & $\sigma=0.006$ & 0.0148 \\
\hline & $R^{2}=0.993$ & $R^{2}=0.992$ & $\mathrm{R}^{2}=0.992$ & $R^{\prime \prime}=0.994$ & & $R^{2}=0.996$ \\
\hline
\end{tabular}




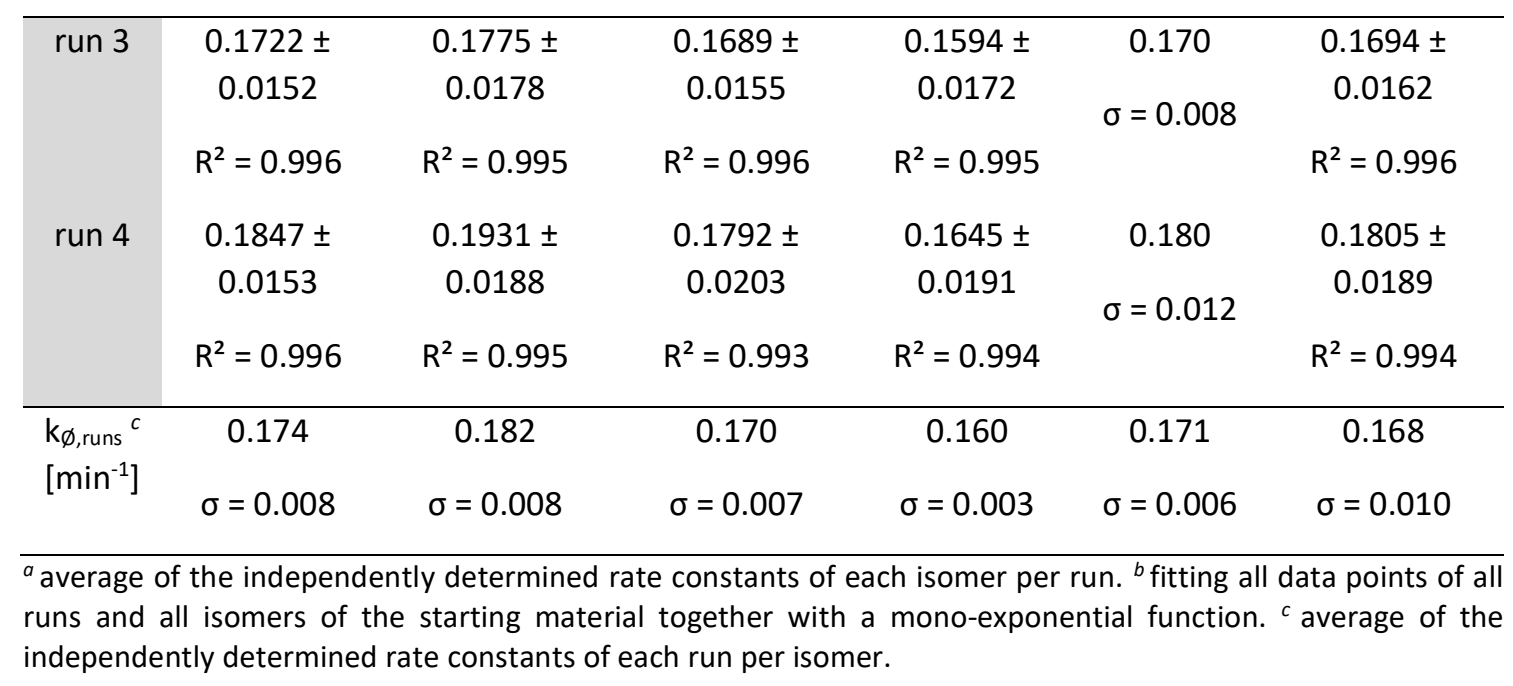

Table S7: Rate constants of the formation of the observed and identified photolysis products of 1-N (diastereomeric mixture A, B, C, D). Experiment run in quartz NMR tube with UV-B-light in ACN- $d_{3}$ under exclusion of air.

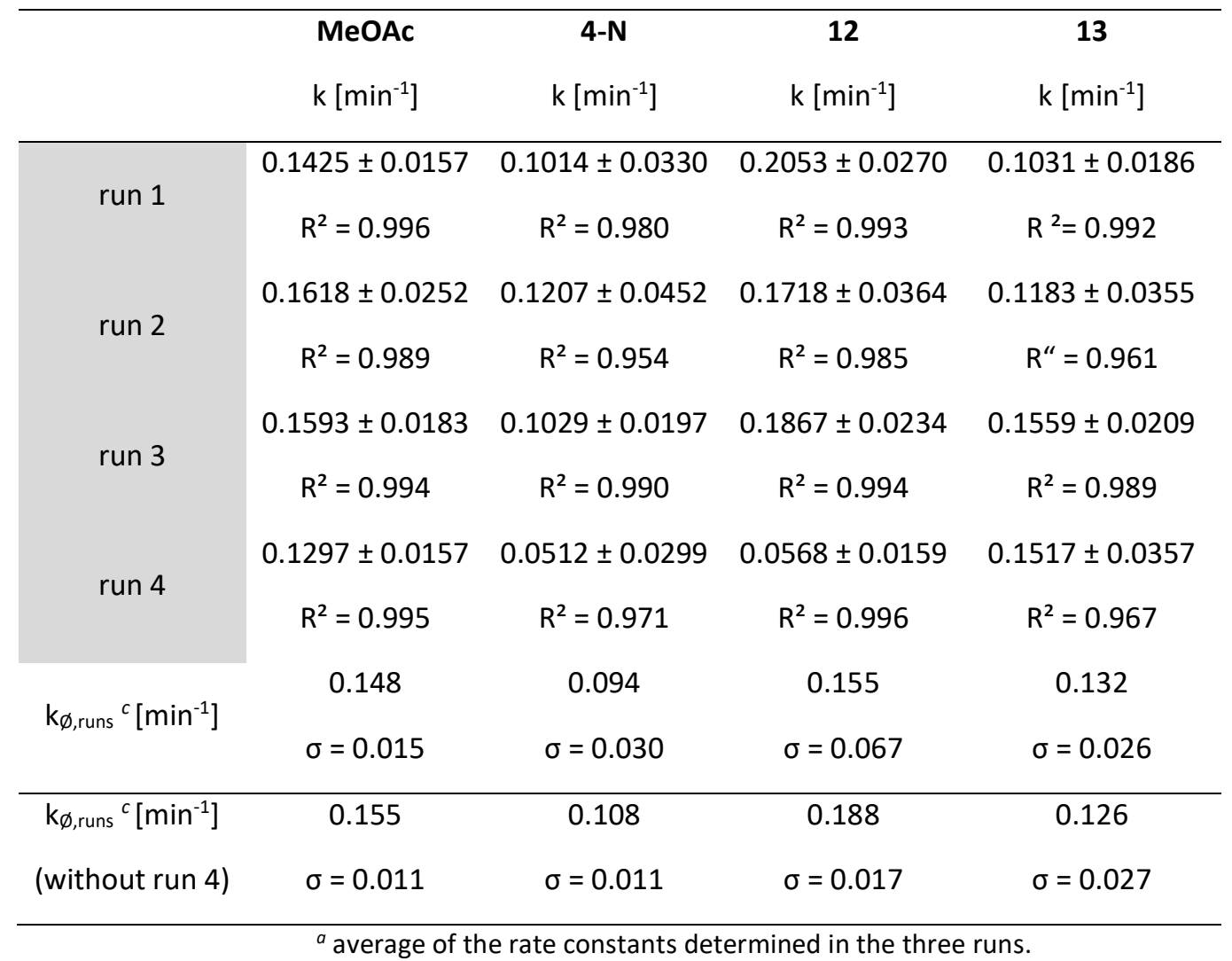




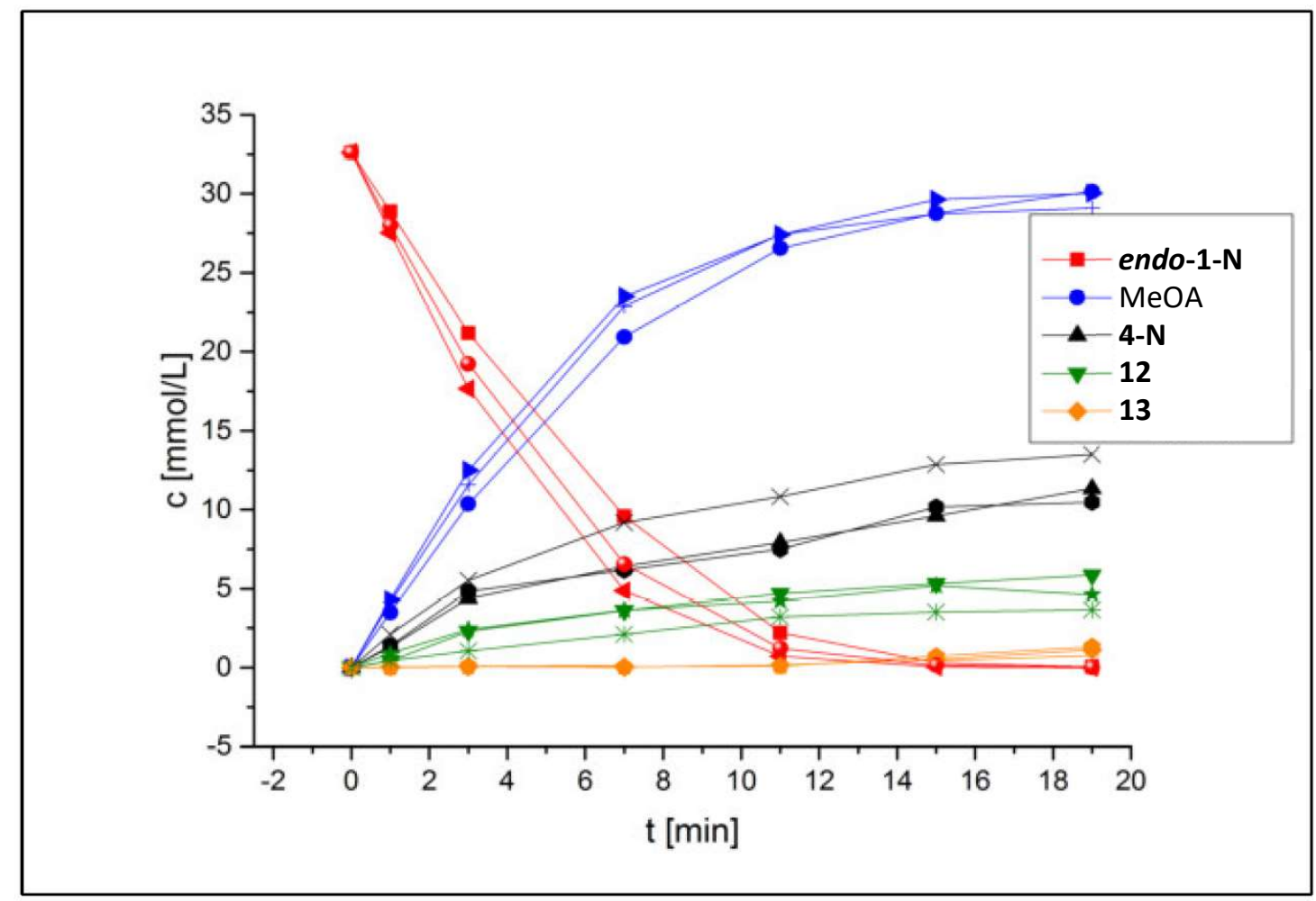

Figure S5: Kinetic trace of the photolysis of endo-1-N in ACN- $\mathrm{d}_{3}$. The photolysis was carried out in a quartz NMR tube with UV-B light under exclusion of air. Rate constants were determined in three independent runs.

Table S8: Concentrations (c) of the observed and identified photolysis products and of endo-1-N as determined by ${ }^{1} \mathrm{H}$ NMR spectroscopy. The photolysis experiments of endo-1-N were run in quartz NMR tubes in degassed ACN- $d_{3}$ (kinetic traces see Figure S2).

\begin{tabular}{ccccccc}
\hline $\begin{array}{c}\mathbf{t} \\
\text { [min] }\end{array}$ & $\begin{array}{c}\text { endo-1-N } \\
\text { run 1 }\end{array}$ & $\begin{array}{c}\text { endo-1-N } \\
\text { run 2 }\end{array}$ & $\begin{array}{c}\text { endo-1-N } \\
\text { run 3 }\end{array}$ & $\begin{array}{c}\text { MeOAc } \\
\text { run 1 }\end{array}$ & $\begin{array}{c}\text { MeOAc } \\
\text { run 2 }\end{array}$ & $\begin{array}{c}\text { MeOAc } \\
\text { run 3 }\end{array}$ \\
\hline 0 & 32.61153 & 32.61153 & 32.61153 & 0 & 0 & 0 \\
1 & 28.87425 & 27.53718 & 28.00352 & 3.48291 & 4.30472 & 4.15471 \\
3 & 21.17141 & 17.66567 & 19.22776 & 10.35742 & 12.50000 & 11.58035 \\
7 & 9.59105 & 4.86890 & 6.55166 & 20.94639 & 23.50639 & 22.89982 \\
11 & 2.21432 & 0.72071 & 1.22619 & 26.54905 & 27.40999 & 27.44260 \\
15 & 0.31307 & 0.05218 & 0.14675 & 28.75685 & 29.63736 & 28.73076 \\
19 & 0.06196 & 0 & 0.01631 & 30.13632 & 30.03196 & 29.09275 \\
\hline $\begin{array}{c}\text { max. } \\
\text { conv. }\end{array}$ & $0.2 \%$ & $0 \%$ & $0 \%$ & $92 \%$ & $92 \%$ & $89 \%$ \\
\hline & & & & & & \\
\hline
\end{tabular}


Table 59. Concentrations (c) of the observed and identified photolysis products and of endo-1-N as determined by ${ }^{1} \mathrm{H}$ NMR spectroscopy. The photolysis experiments of endo-1-N were run in quartz NMR tubes in degassed ACN- $d_{3}$ (kinetic traces see Figure S2).

\begin{tabular}{cccccccccc}
\hline $\begin{array}{c}\mathrm{t} \\
{[\mathrm{min}]}\end{array}$ & $\mathbf{4 - N}$ & $\mathbf{4 - N}$ & $\mathbf{4 - N}$ & $\mathbf{1 2}$ & $\mathbf{1 2}$ & $\mathbf{1 2}$ & $\mathbf{1 3}$ & $\mathbf{1 3}$ & $\mathbf{1 3}$ \\
& run 1 & run 2 & run 3 & run 1 & run 2 & run 3 & run 1 & run 2 & run 3 \\
\hline 0 & 0 & 0 & 0 & 0 & 0 & 0 & 0 & 0 & 0 \\
1 & 1.31424 & 1.38599 & 2.113230 & 0.42069 & 0.90986 & 0.45982 & 0.04892 & 0 & 0 \\
3 & 4.39277 & 4.83303 & 5.53744 & 2.28933 & 2.4263 & 1.04683 & 0.06848 & 0.07827 & 0.09783 \\
7 & 6.41143 & 6.17662 & 9.18015 & 3.60031 & 3.63945 & 2.10344 & 0.02935 & 0.04892 & 0.1174 \\
11 & 7.92786 & 7.48761 & 10.82051 & 4.68628 & 4.21667 & 3.21876 & 0.1761 & 0.10762 & -- \\
15 & 9.59431 & 10.15849 & 12.86199 & 5.3222 & 5.18523 & 3.51226 & 0.52831 & 0.72398 & 0.42069 \\
19 & 11.32598 & 10.47482 & 13.50444 & 5.85051 & 4.61779 & 3.65901 & 1.1251 & 1.32077 & 0.77289 \\
\hline max. & $35 \%$ & $32 \%$ & $41 \%$ & $18 \%$ & $14 \%$ & $11 \%$ & $3 \%$ & $4 \%$ & $2 \%$ \\
conv. & & & & & & & & & \\
\hline
\end{tabular}

Table S10. Rate constants (mono-exponential fit) of the depletion of endo-1-N (diastereomers B and

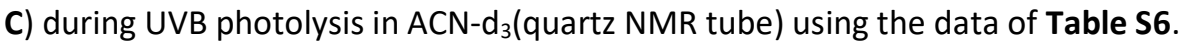

\begin{tabular}{|c|c|c|c|c|}
\hline \multicolumn{3}{|c|}{$\mathrm{k}_{\text {isomer }}\left[\mathrm{min}^{-1}\right]$ of the diastereomers $\mathrm{C}$ and $\mathrm{B}$} & \multirow[b]{2}{*}{$\mathrm{k} \emptyset$, isomers ${ }^{a}\left[\mathrm{~min}^{-1}\right]$} & \multirow[b]{2}{*}{$\mathrm{k}_{\varnothing, \text { total }}{ }^{b}\left[\mathrm{~min}^{-1}\right]$} \\
\hline & R-1 & C-1-N & & \\
\hline \multirow{2}{*}{ run 1} & $0.1470 \pm 0.0147$ & $0.1572 \pm 0.0209$ & \multirow{2}{*}{0.152} & $0.1512 \pm 0.0203$ \\
\hline & $R^{2}=0.996$ & $R^{2}=0.992$ & & $R^{2}=0.992$ \\
\hline \multirow{2}{*}{ run 2} & $0.2190 \pm 0.0247$ & $0.2139 \pm 0.0243$ & 0.216 & $0.2199 \pm 0.0248$ \\
\hline & $R^{2}=0.991$ & $R^{2}=0.992$ & & $R^{2}=0.992$ \\
\hline \multirow{2}{*}{ run 3} & $0.1961 \pm 0.0229$ & $0.1868 \pm 0.0224$ & 0.191 & $0.1912 \pm 0.0223$ \\
\hline & $R^{2}=0.995$ & $R^{2}=0.992$ & & $R^{2}=0.993$ \\
\hline$k_{\emptyset, \text { runs }}{ }^{c}$ & 0.187 & 0.186 & 0.187 & 0.187 \\
\hline$\left[\mathrm{min}^{-1}\right]$ & $\sigma=0.037$ & $\sigma=0.028$ & $\sigma=0.032$ & $\sigma=0.035$ \\
\hline
\end{tabular}

\footnotetext{
${ }^{a}$ average of the independently determined rate constants of each isomer per run. ${ }^{b}$ fitting all data points of all runs and all isomers of the starting material together with a mono-exponential function. ${ }^{c}$ average of the independently determined rate constants of each run per isomer.
} 
Table S11. Rate constants (mono-exponential fit) of the formation of photoproducts during the UVB photolysis of endo-1-N (diastereomers B and C) in degassed ACN-d $\mathrm{d}_{3}$ (quartz NMR tube) using the data of Tables $\mathbf{S 6} \&$ S7.

\begin{tabular}{|c|c|c|c|c|}
\hline & \multicolumn{4}{|c|}{$\mathrm{k}\left[\mathrm{min}^{-1}\right]$} \\
\hline & MeOAc & $4-N$ & 12 & 13 \\
\hline \multirow{2}{*}{ run 1} & $0.1446 \pm 0.0122$ & $0.0960 \pm 0.0299$ & $0.1407 \pm 0.0321$ & $0.2088 \pm 0.0259$ \\
\hline & $R^{2}=0.997$ & $R^{2}=0.977$ & $R^{2}=0.987$ & $R^{2}=0.988$ \\
\hline \multirow{2}{*}{ run 2} & $0.1862 \pm 0.0140$ & $0.1132 \pm 0.0441$ & $0.1691 \pm 0.0425$ & $0.1927 \pm 0.0459$ \\
\hline & $R^{2}=0.997$ & $R^{2}=0.955$ & $R^{2}=0.980$ & $R^{\prime \prime}=0.958$ \\
\hline \multirow{2}{*}{ run 3} & $0.1844 \pm 0.0199$ & $0.1460 \pm 0.0163$ & $0.0790 \pm 0.0255$ & $0.1326 \pm 0.0273$ \\
\hline & $R^{2}=0.994$ & $R^{2}=0.995$ & $R^{2}=0.989$ & $R^{2}=0.988$ \\
\hline \multirow{2}{*}{$\mathrm{k}_{\varnothing, \text { runs }}{ }^{a}\left[\mathrm{~min}^{-1}\right]$} & 0.172 & 0.118 & 0.130 & 0.178 \\
\hline & $\sigma=0.024$ & $\sigma=0.025$ & $\sigma=0.046$ & $\sigma=0.040$ \\
\hline
\end{tabular}

${ }^{a}$ average of the rate constants determined in the three runs.

\subsection{Photolysis of the Bornyl Precursor 1-B in ACN}

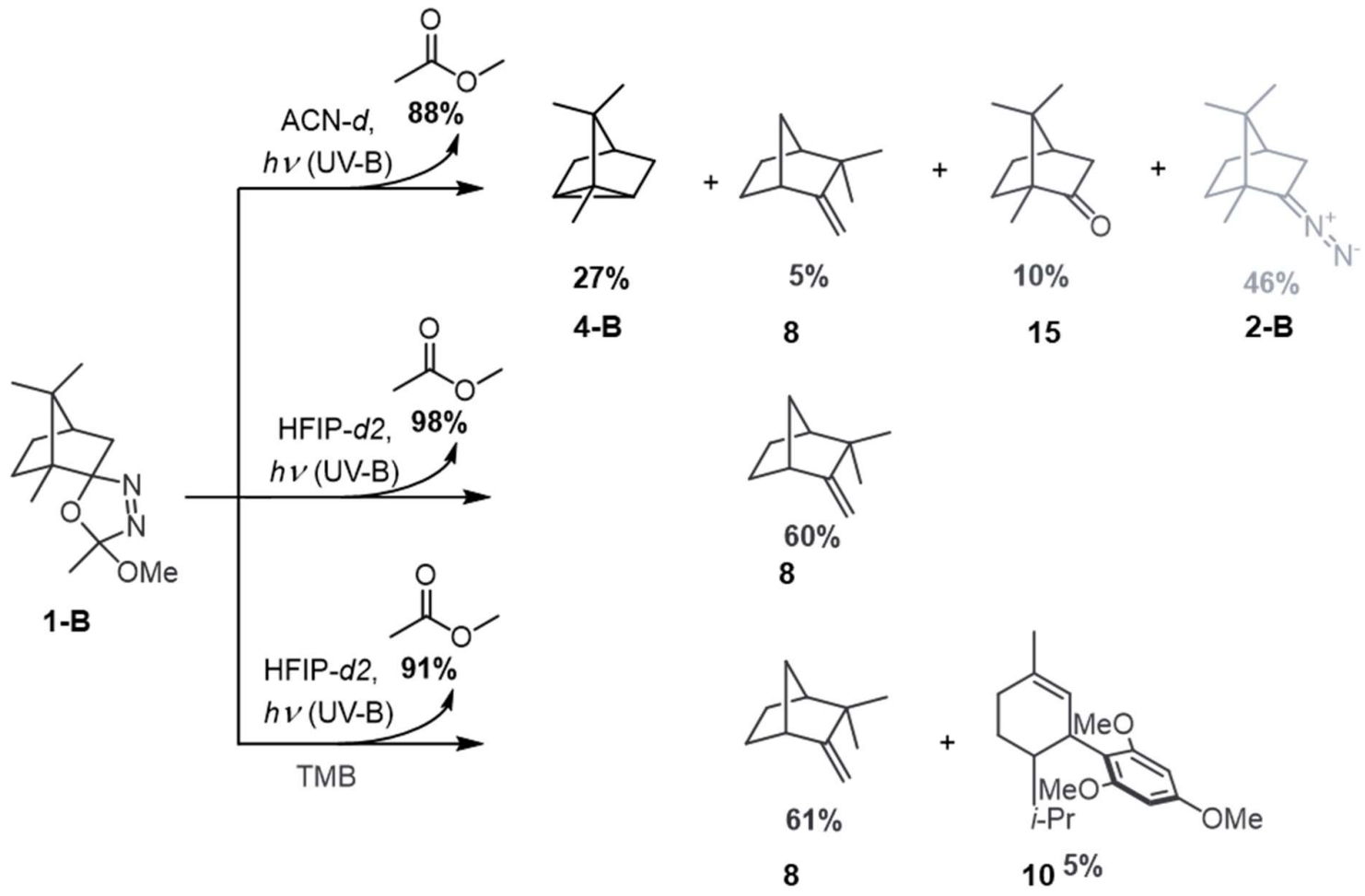

Scheme S5 Overview of products observed and characterized in the UV-B photolysis of 1-B under different conditions. Highlighted is the diazo 2-B that shows unexpected stability under the reaction conditions. 


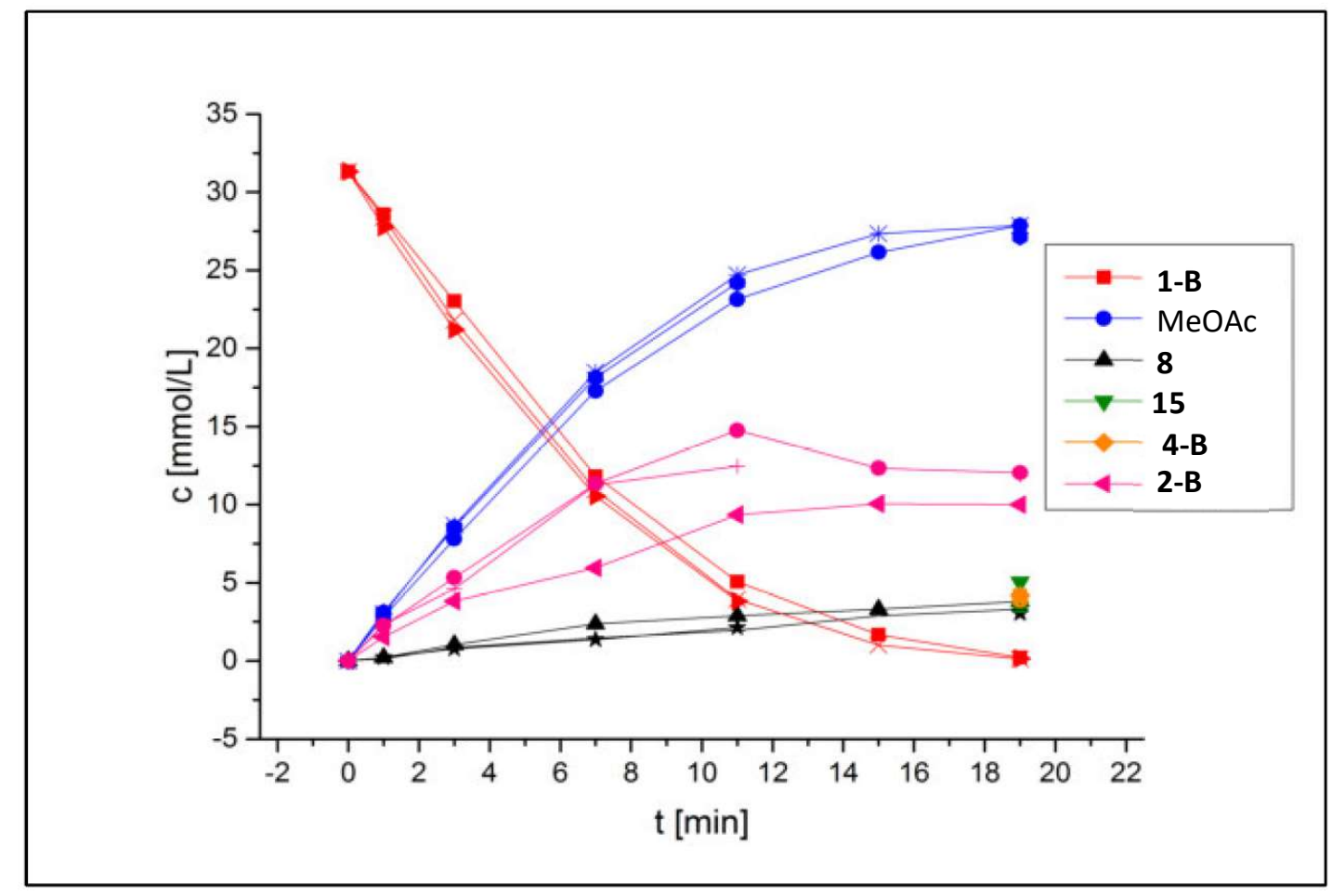

Figure S6. Kinetic trace of the photolysis of 1-B in $A C N-d_{3}$. The photolysis was carried out in quartz NMR tubes with UV-B light (three-fold determination).

Table S12: Concentrations (c) of the reaction products determined by $1 \mathrm{H}$ NMR of the photolysis of 1$B$ in a quartz NMR tube in ACN-d3 (kinetic traces see Figure S3) (1 of 3).

\begin{tabular}{ccccccc}
\hline $\begin{array}{c}\mathrm{t} \\
{[\mathrm{min}]}\end{array}$ & 1-B & 1-B & 1-B & MeOAc & MeOAc & MeOAc \\
& run 1 & run 2 & run 3 & run 1 & run 2 & run 3 \\
\hline 0 & 31.32898 & 31.32898 & 31.32898 & 0 & 0 & 0 \\
1 & 28.56890 & 27.76374 & 28.36212 & 2.82274 & 3.11410 & 3.01698 \\
3 & 23.02367 & 21.20345 & 21.77991 & 7.80405 & 8.46550 & 8.68439 \\
7 & 11.82356 & 10.5516 & 10.92755 & 17.28733 & 18.14574 & 18.48410 \\
11 & 5.07216 & 3.81900 & 3.93805 & 23.13958 & 24.20477 & 24.70917 \\
15 & 1.66670 & - & 1.00566 & 26.14717 & - & 27.33140 \\
19 & 0.22557 & 0.14725 & 0.11905 & 27.87026 & 27.15656 & 27.86713 \\
\hline max. & $1 \%$ & $0.5 \%$ & $0.4 \%$ & $89 \%$ & $87 \%$ & $89 \%$ \\
conv. & & & & & & \\
\hline
\end{tabular}


Table S13. Concentrations (c) of the reaction products determined by $1 \mathrm{H}$ NMR of the photolysis of 1$B$ in a quartz NMR tube in ACN-d3 (kinetic traces see Figure S3) (2 of 3).

\begin{tabular}{ccccccc}
$\mathrm{t}$ & \multicolumn{5}{c}{$\mathrm{c}[\mathrm{mmol} / \mathrm{L}]$} \\
{$[\mathrm{min}]$} & $\mathbf{8}$ & $\mathbf{8}$ & $\mathbf{8}$ & $\mathbf{1 5}$ & $\mathbf{1 5}$ & 15 \\
& run 1 & run 1 & run 1 & run 1 & run 2 & run 3 \\
0 & 0 & 0 & 0 & - & - & - \\
1 & 0.21617 & 0.22557 & 0.15038 & - & - & - \\
3 & 1.04325 & 0.78009 & 0.83648 & - & - & - \\
7 & 2.36847 & 1.36281 & 1.46620 & - & - & - \\
11 & 2.88540 & 2.12410 & 1.95493 & - & - & - \\
15 & 3.30834 & - & 2.87600 & - & - & - \\
19 & 3.80647 & 3.04518 & 3.29894 & 5.03770 & 3.57150 & 4.04144 \\
max. & $12 \%$ & $10 \%$ & $11 \%$ & $16 \%$ & $11 \%$ & $13 \%$ \\
conv. & & & & & &
\end{tabular}

Table S14. Concentrations (c) of the reaction products determined by ${ }^{1} \mathrm{H}$ NMR of the photolysis of 1B in a quartz NMR tube in ACN-d3 (kinetic traces see Figure S3) (3 of 3). ). Last row shows final conversion in $\%$.

\begin{tabular}{ccccccc}
\hline \multirow{t}{*}{} & & \multicolumn{5}{c}{$\mathrm{c}[\mathrm{mmol} / \mathrm{L}]$} \\
{$[\mathrm{min}]$} & 4-B & 4-B (b) & 4-B (c) & 2-B & 2-B & 2-B \\
& run 1 & run 2 & run 3 & run 1 & run 2 & run 3 \\
\hline 0 & - & - & - & 0 & 0 & 0 \\
1 & - & - & - & 1.52259 & 2.33088 & 2.25569 \\
3 & - & - & - & 3.82527 & 4.60536 & 5.32906 \\
7 & - & - & - & 5.93997 & 11.26903 & 11.34422 \\
11 & - & - & - & 9.35170 & 12.46267 & 14.75595 \\
15 & - & - & - & 10.0566 & - & 12.34048 \\
19 & 4.16362 & 3.87226 & 4.16362 & 9.99081 & 11.85175 & 12.03973 \\
\hline max. & $13 \%$ & $12 \%$ & $13 \%$ & $32 \%$ & $38 \%$ & $38 \%$ \\
conv. & & & & & & \\
\hline
\end{tabular}


Table S15. Rate constants k (mono-exponential fit) of the different isomers of 1-B in the photolysis experiment (UVB) in quartz NMR tubes in $A C N-d_{3}$ (degassed).

\begin{tabular}{|c|c|c|c|c|c|c|}
\hline & \multicolumn{4}{|c|}{$\mathrm{K}_{\text {isomer }}\left[\mathrm{min}^{-1}\right]$ of the single diastereomers A - D } & \multirow{2}{*}{$\begin{array}{c}\mathrm{k}_{\emptyset, \text { isomers }}{ }^{a} \\
{\left[\mathrm{~min}^{-1}\right]}\end{array}$} & \multirow{2}{*}{$\begin{array}{c}\mathrm{k}_{\varnothing, \text { total }^{b}}{ }^{b} \\
{\left[\mathrm{~min}^{-1}\right]}\end{array}$} \\
\hline & A-1-B & B-1-B & C-1-B & D-1-B & & \\
\hline run 1 & $\begin{array}{c}0.1234 \pm \\
0.0166 \\
R^{2}=0.994\end{array}$ & $\begin{array}{c}0.1302 \pm \\
0.0156 \\
\mathrm{R}^{2}=0.995\end{array}$ & $\begin{array}{c}0.1122 \pm \\
0.0154 \\
\mathrm{R}^{2}=0.994\end{array}$ & $\begin{array}{c}0.1005 \pm \\
0.0140 \\
R^{2}=0.995\end{array}$ & $\begin{array}{c}0.117 \\
\sigma=0.013\end{array}$ & $\begin{array}{c}0.1100 \pm \\
0.0159 \\
R^{2}=0.994\end{array}$ \\
\hline run 2 & $\begin{array}{c}0.1376 \pm \\
0.0200 \\
R^{2}=0.992\end{array}$ & $\begin{array}{c}0.1584 \pm \\
0.0166 \\
\mathrm{R}^{2}=0.995\end{array}$ & $\begin{array}{c}0.1310 \pm \\
0.0202 \\
\mathrm{R}^{2}=0.991\end{array}$ & $\begin{array}{c}0.1162 \pm \\
0.0100 \\
R^{\prime \prime}=0.998\end{array}$ & $\begin{array}{c}0.136 \\
\sigma=0.018\end{array}$ & $\begin{array}{c}0.1333 \pm \\
0.0160 \\
R^{2}=0.995\end{array}$ \\
\hline run 3 & $\begin{array}{c}0.1356 \pm \\
0.0141 \\
R^{2}=0.996\end{array}$ & $\begin{array}{c}0.1431 \pm \\
0.0206 \\
R^{2}=0.992\end{array}$ & $\begin{array}{c}0.1275 \pm \\
0.0163 \\
R^{2}=0.994\end{array}$ & $\begin{array}{c}0.1182 \pm \\
0.0170 \\
R^{2}=0.993\end{array}$ & $\begin{array}{c}0.131 \\
\sigma=0.011\end{array}$ & $\begin{array}{c}0.1254 \pm \\
0.0161 \\
R^{2}=0.994\end{array}$ \\
\hline $\begin{array}{l}k_{\phi, \text {,runs }}{ }^{c} \\
{\left[\mathrm{~min}^{-1}\right]}\end{array}$ & $\begin{array}{c}0.132 \\
\sigma=0.008\end{array}$ & $\begin{array}{c}0.144 \\
\sigma=0.014\end{array}$ & $\begin{array}{c}0.124 \\
\sigma=0.010\end{array}$ & $\begin{array}{c}0.160 \\
\sigma=0.003\end{array}$ & $\begin{array}{c}0.128 \\
\sigma=0.010\end{array}$ & $\begin{array}{c}0.123 \\
\sigma=0.012\end{array}$ \\
\hline
\end{tabular}

${ }^{a}$ average of the independently determined rate constants of each isomer per run. ${ }^{b}$ fitting all data points of all runs and all isomers of the starting material together with a mono-exponential function. ${ }^{c}$ average of the independently determined rate constants of each run per isomer.

Table S16. Rate constants $k$ (mono-exponential fit) of the different reaction products determined from the ${ }^{1} \mathrm{H}$ NMR monitoring of the photolysis of 1-B in quartz NMR tubes (ACN- $\mathrm{d}_{3}$, degassed, UVB).

\begin{tabular}{cccc}
\hline & & $\mathrm{k}\left[\mathrm{min}^{-1}\right]$ & \\
& MeOAc & $\mathbf{8}$ & 2-B \\
\hline run 1 & $0.1089 \pm 0.0114$ & $0.1041 \pm 0.0178$ & $0.1271 \pm 0.0317$ \\
& $\mathrm{R}^{2}=0.997$ & $\mathrm{R}^{2}=0.992$ & $\mathrm{R}^{2}=0.978$ \\
run 2 & $0.1327 \pm 0.0194$ & $0.0466 \pm 0.0115$ & $0.2203 \pm 0.0723$ \\
& $\mathrm{R}^{2}=0.992$ & $\mathrm{R}^{2}=0.995$ & $\mathrm{R}^{2}=0.944$ \\
run 3 & $0.1288 \pm 0.0167$ & $0.0282 \pm 0.0192$ & $0.2388 \pm 0.0841$ \\
& $\mathrm{R}^{2}=0.994$ & $\mathrm{R}^{2}=0.987$ & $\mathrm{R}^{2}=0.925$ \\
\hline $\mathrm{k}_{\varnothing \text {, runs }}{ }^{a}\left[\mathrm{~min}^{-1}\right]$ & 0.123 & 0.059 & 0.195 \\
& $\sigma=0.013$ & $\sigma=0.040$ & $\sigma=0.060$ \\
\hline
\end{tabular}

${ }^{a}$ average of the rate constants determined in the three runs. 
2.3 Photolysis of Fenchyl Precursor 1-F in ACN

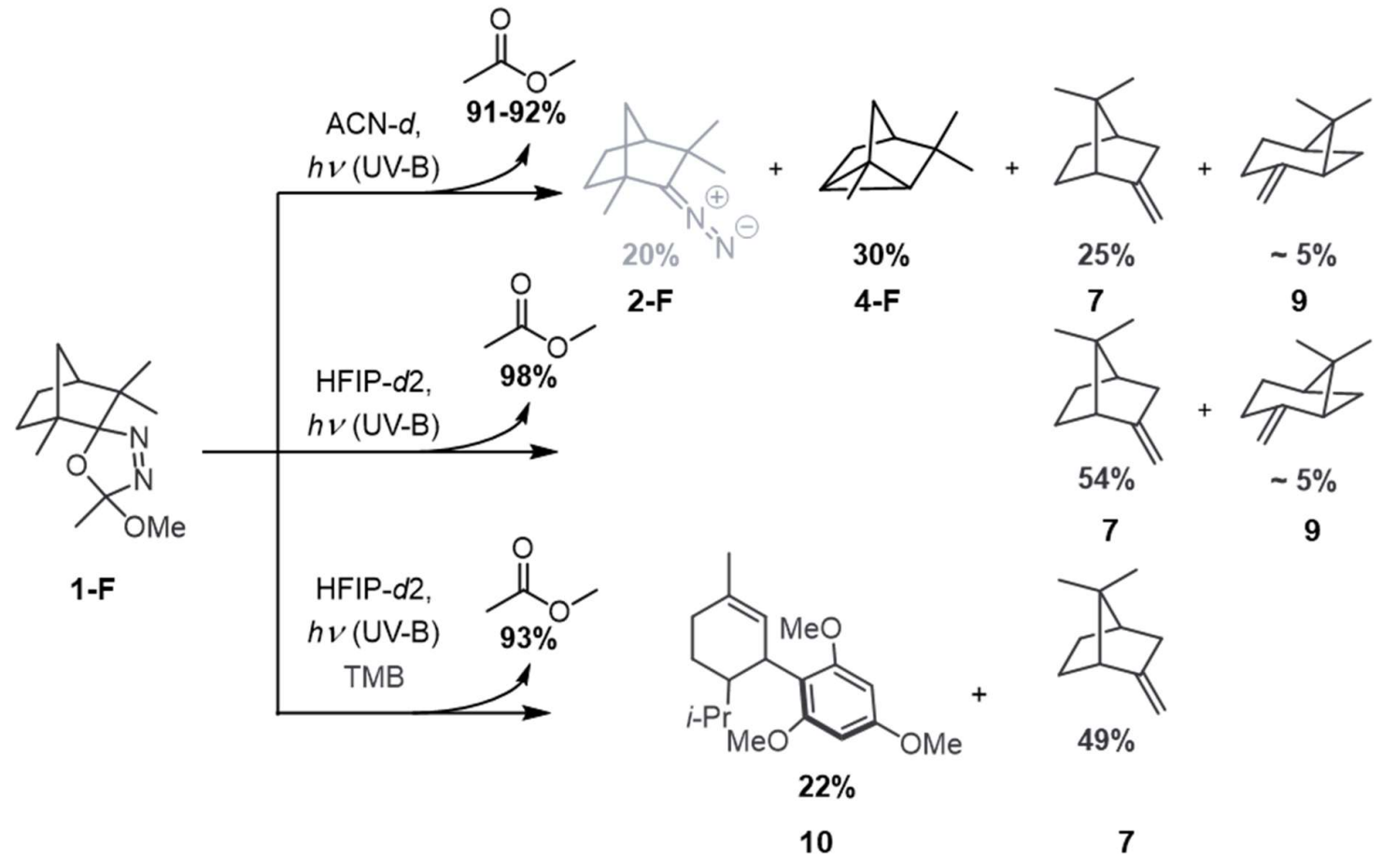

Scheme S6 Overview of products observed and characterized in the UV-B photolysis of 1-F under different conditions. The under these reaction conditions unexpectedly stable $\mathbf{2 - F}$ is highlighted.

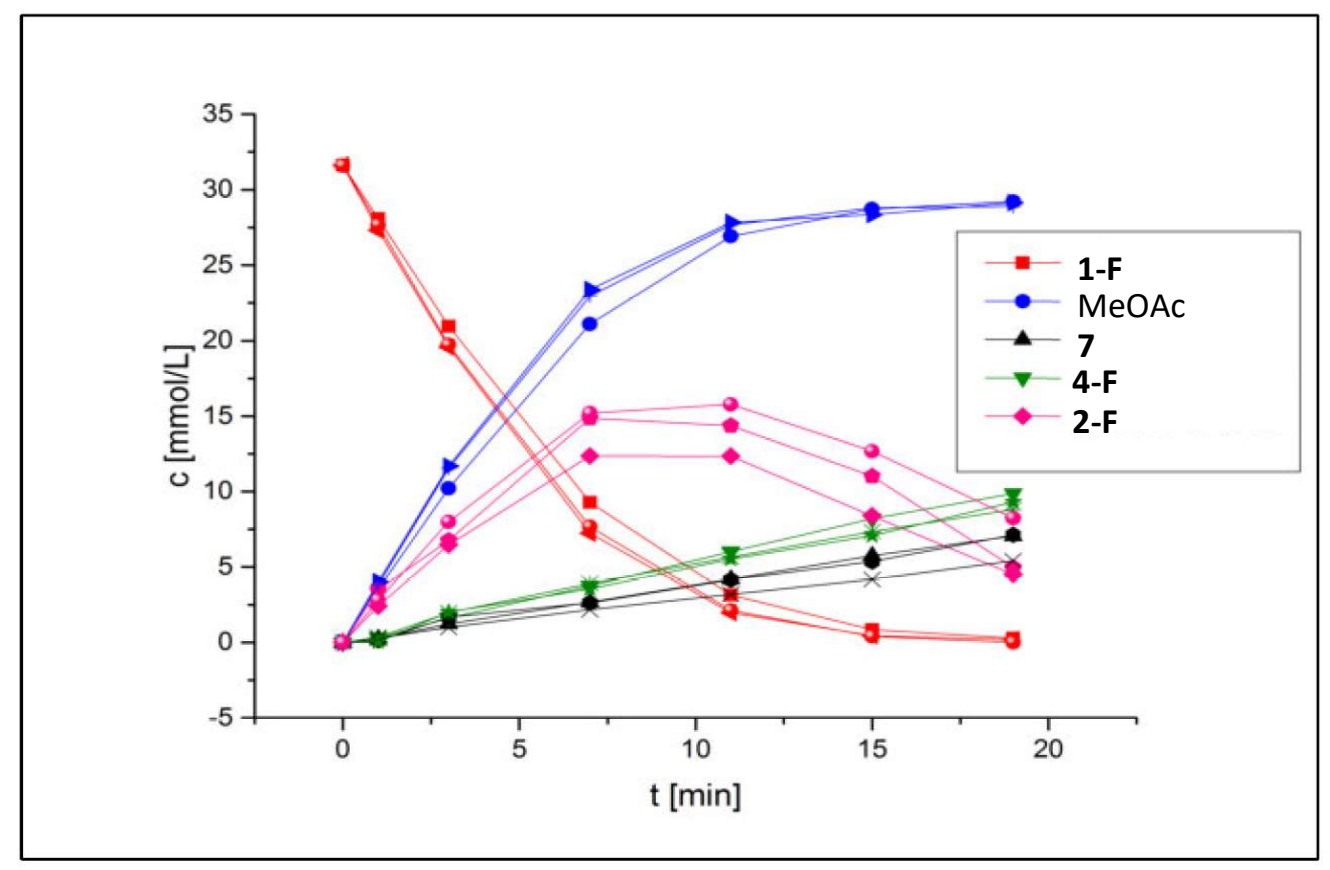

Figure S7. Kinetic trace of the photolysis of 1-F in $A C N-d_{3}$. The photolysis was carried out in a quartz NMR tubes with UV-B light in three independent runs. 
Table S17. Concentration (c) changes in dependence of time of 1-F and MeOAc determined by ${ }^{1} \mathrm{H}$ NMR of the photolysis of 1-F in a quartz NMR tubes in ACN- $\mathrm{d}_{3}$ (plotted kinetic traces see Figure S4). Last row shows final conversion in $\%$.

\begin{tabular}{c|cccccc}
\hline & \multicolumn{5}{|c}{$c[\mathrm{mmol} / \mathrm{L}]$} \\
$\mathrm{t}$ & $\mathbf{1 - F}$ & $\mathbf{1 - F}$ & $\mathbf{1 - F}$ & MeOAc & MeOAc & MeOAc \\
& run 1 & run 2 & run 3 & run 1 & run 2 & run 3 \\
\hline 0 & 31.60870 & 31.60870 & 31.60870 & 0 & 0 & 0 \\
1 & 28.07169 & 27.32256 & 27.67342 & 3.55282 & 4.02695 & 3.83414 \\
3 & 20.96289 & 19.57527 & 19.73015 & 10.21593 & 11.67941 & 11.5593 \\
7 & 9.27083 & 7.23207 & 7.66827 & 21.10829 & 23.39044 & 22.96372 \\
11 & 3.14190 & 1.96606 & 2.12094 & 23.91481 & 27.84094 & 27.67658 \\
15 & 0.85027 & 0.44884 & 0.40775 & 28.72283 & 28.36249 & 28.79553 \\
19 & 0.28764 & 0.19281 & 0.01580 & 29.22224 & 29.14006 & 28.89983 \\
\hline $\begin{array}{c}\text { max. } \\
\text { conv. }\end{array}$ & $1 \%$ & $1 \%$ & $0 \%$ & $92 \%$ & $92 \%$ & $91 \%$ \\
\hline
\end{tabular}

Table S18. Concentrations (c) of the reaction products $\mathbf{7}$ and $\mathbf{4}$ determined by ${ }^{1} \mathrm{H}$ NMR of the photolysis (UVB) of 1-F in a quartz NMR tubes in $\mathrm{ACN}-\mathrm{d}_{3}$ (plotted kinetic traces see Figure S4). Last row shows final conversion in \%.

\begin{tabular}{c|cccccc}
\hline $\mathrm{t}$ & \multicolumn{6}{c}{$\mathrm{c}[\mathrm{mmol} / \mathrm{L}]$} \\
{$[\mathrm{min}]$} & $\mathbf{7}$ & $\mathbf{7}$ & $\mathbf{7}$ & $\mathbf{4 - F}$ & $\mathbf{4 - F}$ & $\mathbf{4 - F}$ \\
& run 1 & run 2 & run 3 & run 1 & run 2 & run 3 \\
\hline 0 & 0 & 0 & 0 & 0 & 0 & 0 \\
1 & 0.27500 & 0.13276 & 0.28448 & 0.30344 & 0.03793 & 0.36034 \\
3 & 1.22326 & 1.67842 & 1.01464 & 1.61204 & 2.02928 & 1.97238 \\
7 & 2.67410 & 2.63617 & 2.17152 & 3.75511 & 3.55598 & 3.90684 \\
11 & 4.19131 & 4.14390 & 3.17667 & 6.03094 & 5.52836 & 5.65164 \\
15 & 5.75594 & 5.36716 & 4.17235 & 8.20246 & 7.07403 & 7.34902 \\
19 & 7.06454 & 7.12144 & 5.40509 & 9.86191 & 9.30244 & 8.79986 \\
\hline max. & $22 \%$ & $23 \%$ & $17 \%$ & $31 \%$ & $29 \%$ & $28 \%$ \\
conv. & & & & & & \\
\hline
\end{tabular}


Table S19. Concentrations (c) of product 2-F in dependence of time determined by ${ }^{1} \mathrm{H}$ NMR of the photolysis of 1-F in a quartz NMR tubes in ACN- $d_{3}$ (plotted kinetic traces see Figure $S 4$ ). Last row shows final conversion in \%.

\begin{tabular}{c|ccc}
\hline $\begin{array}{c}\mathrm{t} \\
{[\mathrm{min}]}\end{array}$ & $\mathbf{3}$ & $\mathrm{c}[\mathrm{mmol} / \mathrm{L}]$ & $\mathbf{2 - F}$ \\
\hline 0 & 0 & 0 & $\mathbf{2 - F}$ \\
1 & 2.41332 & 3.49592 & 2.81634 \\
3 & 6.4782 & 6.77216 & 7.98752 \\
7 & 12.36216 & 14.86557 & 15.21169 \\
11 & 12.3511 & 14.3788 & 15.77906 \\
15 & 8.38579 & 11.01405 & 12.67983 \\
19 & 4.51846 & 4.97837 & 8.24039 \\
\hline max. & $39 \%$ & $47 \%$ & $50 \%$ \\
conv. & & & \\
\hline
\end{tabular}

Table S20. Rate constants k (mono-exponential fit) of the different isomers of 1-F in the photolysis experiment (UVB) in quartz NMR tubes in ACN- $d_{3}$.

\begin{tabular}{|c|c|c|c|c|c|c|}
\hline & \multicolumn{4}{|c|}{$\mathrm{k}\left[\mathrm{min}^{-1}\right]$ single diastereomers A-D } & \multirow{2}{*}{$\begin{array}{c}\mathrm{k} \varnothing \text {,isomers }{ }^{a} \\
{\left[\mathrm{~min}^{-1}\right]}\end{array}$} & \multirow{2}{*}{$\begin{array}{l}\mathrm{k} \varnothing, \text { total }^{b} \\
{\left[\mathrm{~min}^{-1}\right]}\end{array}$} \\
\hline & $A-1-F$ & B-1-F & C-1-F & D-1-F & & \\
\hline run 1 & $\begin{array}{c}0.1456 \pm \\
0.0236 \\
R^{2}=0.989\end{array}$ & $\begin{array}{c}0.1374 \pm \\
0.0169 \\
R^{2}=0.994\end{array}$ & $\begin{array}{c}0.1573 \pm \\
0.0188 \\
R^{2}=0.994\end{array}$ & $\begin{array}{c}0.1496 \pm \\
0.0238 \\
\mathrm{R}^{2}=0.990\end{array}$ & $\begin{array}{c}0.147 \\
\sigma=0.008\end{array}$ & $\begin{array}{c}0.1489 \pm \\
0.0179 \\
R^{2}=0.994\end{array}$ \\
\hline run 2 & $\begin{array}{c}0.1543 \pm \\
0.0235 \\
R^{2}=0.990\end{array}$ & $\begin{array}{c}0.1641 \pm \\
0.0208 \\
R^{2}=0.992\end{array}$ & $\begin{array}{c}0.1871 \pm \\
0.0220 \\
R^{2}=0.993\end{array}$ & $\begin{array}{c}0.1871 \pm \\
0.0199 \\
R^{\prime \prime}=0.994\end{array}$ & $\begin{array}{c}0.173 \\
\sigma=0.017\end{array}$ & $\begin{array}{c}0.1770 \pm \\
0.0210 \\
R^{2}=0.993\end{array}$ \\
\hline run 3 & $\begin{array}{c}0.434 \pm \\
0.0296 \\
R^{2}=0.983\end{array}$ & $\begin{array}{c}0.1581 \pm \\
0.0203 \\
R^{2}=0.992\end{array}$ & $\begin{array}{c}0.1820 \pm \\
0.0207 \\
R^{2}=0.993\end{array}$ & $\begin{array}{c}0.1898 \pm \\
0.0154 \\
R^{2}=0.996\end{array}$ & $\begin{array}{c}0.168 \\
\sigma=0.021\end{array}$ & $\begin{array}{c}0.1701 \pm \\
0.0194 \\
R^{2}=0.994\end{array}$ \\
\hline$k_{\varnothing, \text { runs }}{ }^{c}$ & $\begin{array}{c}0.148 \\
\sigma=0.006\end{array}$ & $\begin{array}{c}0.153 \\
\sigma=0.014\end{array}$ & $\begin{array}{c}0.175 \\
\sigma=0.016\end{array}$ & $\begin{array}{c}0.176 \\
\sigma=0.022\end{array}$ & $\begin{array}{c}0.163 \\
\sigma=0.014\end{array}$ & $\begin{array}{c}0.165 \\
\sigma=0.015\end{array}$ \\
\hline
\end{tabular}

${ }^{a}$ average of the independently determined rate constants of each isomer per run. ${ }^{b}$ fitting all data points of all runs and all isomers of the starting material together with a mono-exponential function. ${ }^{c}$ average of the independently determined rate constants of each run per isomer. 
Table S21. Rate constants k (mono-exponential fit) of the different reaction products of the photolysis (UVB) of 1-F in quartz NMR tubes in $\mathrm{ACN}-\mathrm{d}_{3}$.

\begin{tabular}{c|ccc}
\hline & \multicolumn{3}{|c}{$k\left[\mathrm{~min}^{-1}\right]$} \\
& MeOAc & 8 & 2-B \\
\hline run 1 & $0.1543 \pm 0.0185$ & n. d. & n. $d$. \\
& $\mathrm{R}^{2}=0.994$ & (linear) & (linear) \\
run 2 & $0.1918 \pm 0.0244$ & n. d. & n. d. \\
& $\mathrm{R}^{2}=0.991$ & (linear) & (linear) \\
run 3 & $0.1866 \pm 0.0230$ & n. d. & n. d. \\
& $\mathrm{R}^{2}=0.992$ & (linear) & (linear) \\
\hline \multirow{2}{*}{$\mathrm{k}_{\varnothing, \text {,uns }}{ }^{a}\left[\mathrm{~min}^{-1}\right]$} & 0.178 & n. d. & n. d. \\
& $\sigma=0.020$ & & \\
\hline
\end{tabular}

${ }^{a}$ average of the rate constants determined in the three runs.

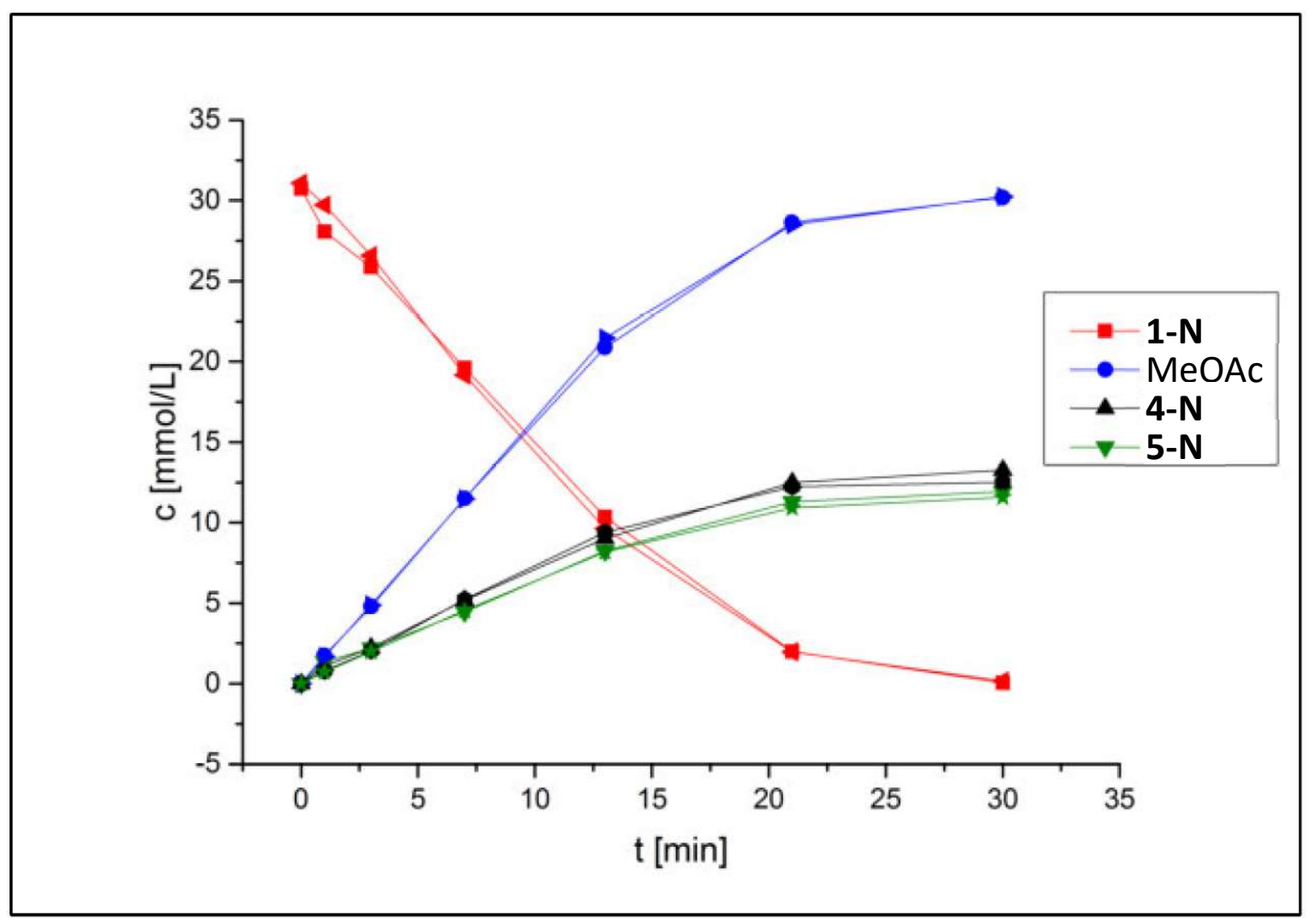

Figure S8. Kinetic trace of the photolysis of 1-N in ACN- $d_{3}$ in the presence of 2 equiv. of TFA. The photolysis was carried out in quartz fluorescence cuvettes $(1 \mathrm{~cm}$ path length) with UV-B light (two independent runs). 
Table S22. Concentrations (c) of the reaction products determined by ${ }^{1} \mathrm{H}$ NMR of the photolysis of 1-N in a quartz cuvette in presence of 2 equiv. of TFA in ACN-d3 (kinetic traces see Figure S5).

\begin{tabular}{|c|c|c|c|c|c|c|c|c|}
\hline \multirow{3}{*}{$\begin{array}{c}\mathrm{t} \\
{[\mathrm{min}]}\end{array}$} & \multicolumn{8}{|c|}{$\mathrm{c}[\mathrm{mmol} / \mathrm{L}]$} \\
\hline & $1-N$ & $1-N$ & MeOAc & MeOAc & $4-N$ & 4-N & $5-N$ & $5-N$ \\
\hline & run 1 & run 2 & run 1 & run 2 & run 1 & run 2 & run 1 & run 2 \\
\hline 0 & 30.74274 & 31.08293 & 0 & 0 & 0 & 0 & 0 & 0 \\
\hline 1 & 28.06813 & 29.72461 & 1.74926 & 1.68159 & 1.11596 & 0.78951 & 1.36498 & 0.73667 \\
\hline 3 & 25.85772 & 26.57901 & 4.78665 & 4.88313 & 2.22577 & 2.04215 & 2.20425 & 2.00485 \\
\hline 7 & 19.60157 & 19.17817 & 11.50394 & 11.48203 & 5.18323 & 5.23437 & 4.4454 & 4.55054 \\
\hline 13 & 10.34801 & 9.62327 & 20.88662 & 21.45655 & 9.02914 & 9.38394 & 8.23598 & 8.16859 \\
\hline 21 & 1.99213 & 1.97687 & 28.64302 & 28.49994 & 12.49693 & 12.23735 & 11.28874 & 10.91011 \\
\hline 30 & 0.03997 & 0.16163 & 30.1863 & 30.24058 & 13.27164 & 12.5171 & 11.93433 & 11.57217 \\
\hline $\begin{array}{l}\text { max. } \\
\text { conv. }\end{array}$ & $0.1 \%$ & $0.5 \%$ & $98 \%$ & $97 \%$ & $43 \%$ & $40 \%$ & $39 \%$ & $37 \%$ \\
\hline
\end{tabular}

\subsection{Photolysis of 1-N in ACN + Acid}

Table S23. Rate constants k (mono-exponential fit) of the different diastereomers A-D of 1-N in the photolysis experiments (UVB) in quartz cuvettes (fluorescence cell, path length $1 \mathrm{~cm}$ ) in presence of 2 equiv. of TFA in $\mathrm{ACN}-\mathrm{d}_{3}$ (kinetic traces see Figure $\mathrm{S} 5$ ).

\begin{tabular}{|c|c|c|c|c|c|c|}
\hline & \multicolumn{4}{|c|}{$k\left[\mathrm{~min}^{-1}\right]$ of the single diastereomers A-D } & \multirow{2}{*}{$\begin{array}{c}\mathrm{k}_{\varnothing \text {,isomers }}{ }^{a} \\
{\left[\mathrm{~min}^{-1}\right]}\end{array}$} & \multirow{2}{*}{$\begin{array}{l}\mathrm{k} \phi, \text { total }^{b} \\
{\left[\mathrm{~min}^{-1}\right]}\end{array}$} \\
\hline & $A-1-N$ & B-1-N & C-1-N & D-1-N & & \\
\hline \multirow{3}{*}{ run 1} & $0.0589 \pm$ & $0.0596 \pm$ & $0.0552 \pm$ & $0.0482 \pm$ & \multirow{3}{*}{$\begin{array}{c}0.056 \\
\sigma=0.005\end{array}$} & $0.0554 \pm$ \\
\hline & 0.0119 & 0.0134 & 0.0130 & 0.0142 & & 0.0131 \\
\hline & $R^{2}=0.990$ & $R^{2}=0.988$ & $R^{2}=0.988$ & $\mathrm{R}^{2}=0.985$ & & $R^{2}=0.988$ \\
\hline \multirow[t]{2}{*}{ run 2} & $\begin{array}{c}0.0701 \pm \\
0.0106\end{array}$ & $\begin{array}{c}0.0678 \pm \\
0.0130\end{array}$ & $\begin{array}{c}0.0631 \pm \\
0.0129\end{array}$ & $\begin{array}{c}0.0510 \pm \\
0.0187\end{array}$ & \multirow{2}{*}{$\begin{array}{c}0.063 \\
\sigma=0.009\end{array}$} & $\begin{array}{c}0.0635 \pm \\
0.0128\end{array}$ \\
\hline & $R^{2}=0.993$ & $R^{2}=0.989$ & $R^{2}=0.989$ & $R^{\prime \prime}=0.975$ & & $R^{2}=0.989$ \\
\hline $\mathrm{k}_{\emptyset \text {,runs }}{ }^{c}$ & 0.065 & 0.064 & 0.059 & 0.050 & 0.060 & 0.060 \\
\hline
\end{tabular}


Table S24. Rate constants k (mono-exponential fit) of the different reaction products of the photolysis of 1-N in the photolysis experiment in the cuvette with UV-B-light in the presence of 2 equiv. TFA in $A C N-d_{3}$ (kinetic traces see Figure $\left.S 5\right)$.

\begin{tabular}{cccc}
\hline & \multicolumn{3}{c}{$\mathrm{k}\left[\mathrm{min}^{-1}\right]$} \\
MeOAc & 4-N & 5-N \\
\hline run 1 & $0.0626 \pm 0.0130$ & $0.0613 \pm 0.0118$ & $0.0595 \pm 0.0146$ \\
& $\mathrm{R}^{2}=0.988$ & $\mathrm{R}^{2}=0.990$ & $\mathrm{R}^{2}=0.985$ \\
run 2 & $0.0648 \pm 0.0132$ & $0.0744 \pm 0.0158$ & $0.0661 \pm 0.0118$ \\
& $\mathrm{R}^{2}=0.988$ & $\mathrm{R}^{2}=0.985$ & $\mathrm{R}^{2}=0.991$ \\
\hline $\mathrm{k} \emptyset$,runs $^{a}\left[\mathrm{~min}^{-1}\right]$ & 0.064 & 0.068 & 0.063 \\
\hline \multicolumn{4}{c}{ average of the rate constants determined in the two runs. }
\end{tabular}

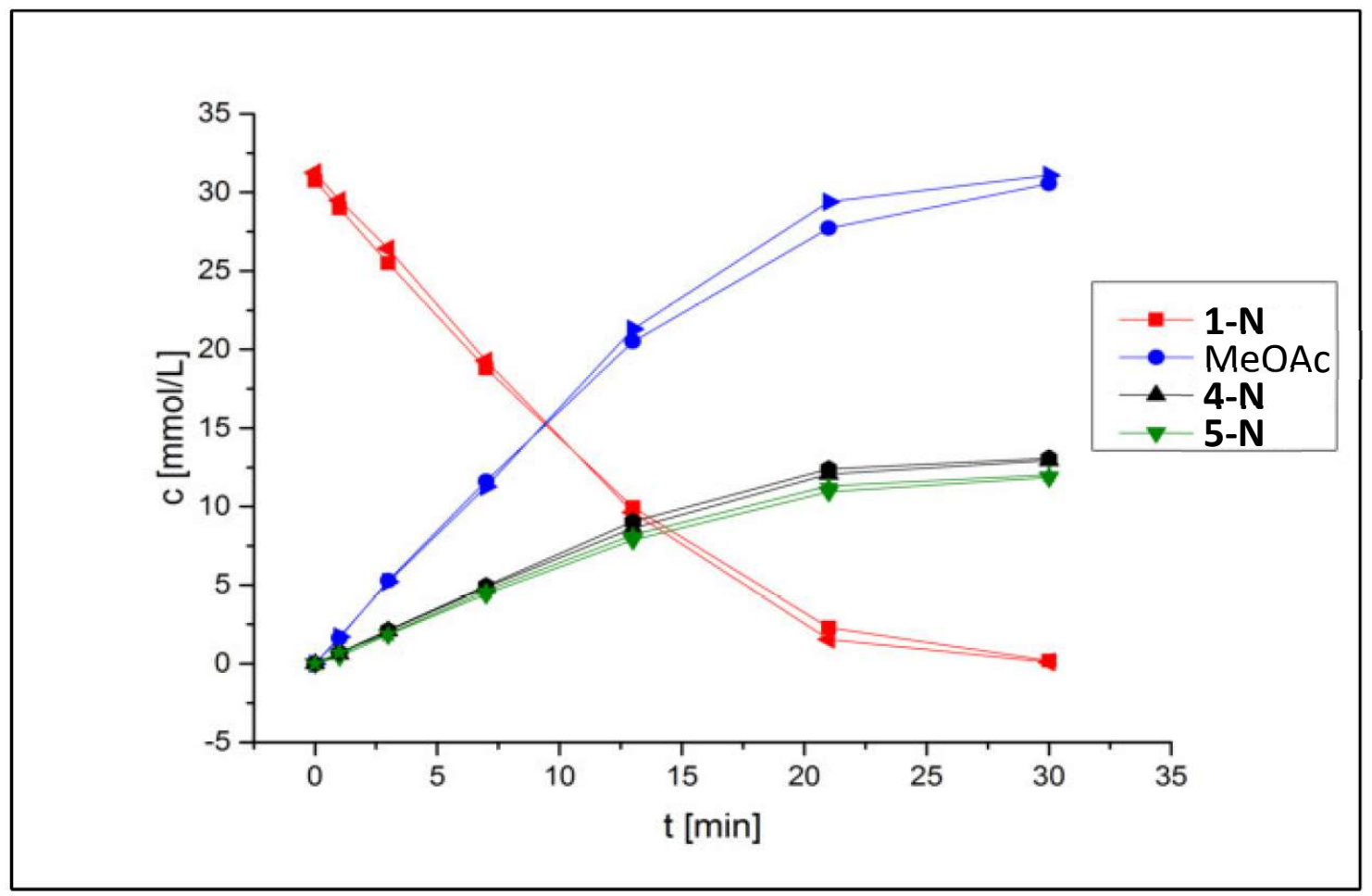

Figure S9. Kinetic trace derived of the ${ }^{1} \mathrm{H}-\mathrm{NMR}$ monitoring of the photolysis (UVB) of 1-N in $\mathrm{ACN}-\mathrm{d}_{3}$ in the presence of 2 equiv. of TFA and 3 equiv. of TMB. The photolysis was carried out in quartz cuvettes (fluorescence cell, path length $1 \mathrm{~cm}$ ) in two independent runs. 
Table S25. Concentrations (c) of the starting materials (summation over all diastereomers) and reaction products determined by ${ }^{1} \mathrm{H}$ NMR of the photolysis of 1-N in quartz cuvettes (fluorescence cell, $1 \mathrm{~cm}$ path length) in presence of 2 equiv. of TFA and 3 equiv. of TMB in $A C N-d_{3}$ (kinetic traces see Figure S6).

\begin{tabular}{c|cccccccc}
\hline & & & \multicolumn{7}{c}{$c[\mathrm{mmol} / \mathrm{L}]$} \\
$\mathrm{t}$ & $\mathbf{1 - N}$ & $\mathbf{1 - N}$ & MeOAc & MeOAc & $\mathbf{4 - N}$ & $\mathbf{4 - N}$ & $\mathbf{5 - N}$ & $\mathbf{5 - N}$ \\
{$[\mathrm{min}]$} & & & & & & & & \\
& run 1 & run 2 & run 1 & run 2 & run 1 & run 2 & run 1 & run 2 \\
\hline 0 & 30.74274 & 31.25266 & 0 & 0 & 0 & 0 & 0 & 0 \\
1 & 28.98119 & 29.48376 & 1.63859 & 1.72202 & 0.60256 & 0.70943 & 0.47036 & 0.72194 \\
3 & 25.47959 & 26.41475 & 5.27853 & 5.19106 & 2.08128 & 2.12518 & 1.84456 & 1.89391 \\
7 & 18.78689 & 19.28289 & 11.60539 & 11.27283 & 4.84813 & 4.94729 & 4.43618 & 4.63164 \\
13 & 9.96065 & 9.61644 & 20.50848 & 21.29867 & 8.62334 & 9.05076 & 7.86707 & 8.19444 \\
21 & 2.29956 & 1.55326 & 27.72073 & 29.39623 & 12.06345 & 12.41042 & 10.94749 & 11.3072 \\
30 & 0.18446 & 0.10313 & 30.55521 & 31.08700 & 12.93962 & 13.09173 & 11.86055 & 12.02914 \\
\hline $\begin{array}{c}\text { max. } \\
\text { conv. }\end{array}$ & $0.6 \%$ & $0.3 \%$ & $99 \%$ & $99 \%$ & $42 \%$ & $42 \%$ & $39 \%$ & $38 \% 11$ \\
\hline
\end{tabular}

Table S26. Rate constants $k$ (mono-exponential fit) of the different diastereomers of 1-N in the photolysis experiment in the quartz cuvette with UV-B light in presence of 2 equiv. of TFA and 3 equiv. of $T M B$ in $A C N-d_{3}$ (kinetic traces see Figure 56 ).

\begin{tabular}{|c|c|c|c|c|c|c|}
\hline & \multicolumn{4}{|c|}{$\mathrm{k}\left[\mathrm{min}^{-1}\right]$ of the single diastereomers A-D } & \multirow{2}{*}{$\begin{array}{c}\mathrm{k}_{\emptyset \text {,isomers }}{ }^{a} \\
{\left[\mathrm{~min}^{-1}\right]}\end{array}$} & \multirow{2}{*}{$\begin{array}{l}\mathrm{k} \phi, \text { total } \\
{\left[\mathrm{min}^{-1}\right]}\end{array}$} \\
\hline & A-1-N & B-1-N & C-1-N & $D-1-N$ & & \\
\hline run 1 & $\begin{array}{c}0.0628 \pm \\
0.0099 \\
R^{2}=0.993\end{array}$ & $\begin{array}{c}0.0650 \pm \\
0.0103 \\
R^{2}=0.993\end{array}$ & $\begin{array}{c}0.0625 \pm \\
0.0097 \\
R^{2}=0.994\end{array}$ & $\begin{array}{c}0.0592 \pm \\
0.0099 \\
R^{2}=0.993\end{array}$ & $\begin{array}{c}0.062 \\
\sigma=0.002\end{array}$ & $\begin{array}{c}0.0626 \pm \\
0.0099 \\
R^{2}=0.993\end{array}$ \\
\hline run 2 & $\begin{array}{c}0.0667 \pm \\
0.0121 \\
R^{2}=0.990\end{array}$ & $\begin{array}{c}0.0658 \pm \\
0.0135 \\
R^{2}=0.988\end{array}$ & $\begin{array}{c}0.0615 \pm \\
0.0156 \\
R^{2}=0.983\end{array}$ & $\begin{array}{c}0.0603 \pm \\
0.0129 \\
R^{\prime \prime}=0.988\end{array}$ & $\begin{array}{c}0.064 \\
\sigma=0.003\end{array}$ & $\begin{array}{c}0.0634 \pm \\
0.0135 \\
R^{2}=0.988\end{array}$ \\
\hline $\begin{array}{c}\mathrm{k}_{\emptyset, \text {,isomers }}{ }^{a} \\
{\left[\mathrm{~min}^{-1}\right]}\end{array}$ & 0.065 & 0.065 & 0.062 & 0.060 & 0.063 & 0.063 \\
\hline
\end{tabular}


Table S27. Rate constants $k$ (mono-exponential fit) of the different reaction products of the UVBphotolysis of 1-N in a quartz cuvette (fluorescence cell, path length $1 \mathrm{~cm}$ ) in the presence of 2 equiv. TFA and 3 equiv. TMB in ACN- $d_{3}$ (kinetic traces see Figure $\mathrm{S} 6$ ).

\begin{tabular}{cccc}
\hline & \multicolumn{4}{c}{$\mathrm{k}\left[\mathrm{min}^{-1}\right]$} \\
& MeOAc & 4-N & $\mathbf{5 - N}$ \\
\hline run 1 & $0.0626 \pm 0.0130$ & $0.0613 \pm 0.0118$ & $0.0595 \pm 0.0146$ \\
& $\mathrm{R}^{2}=0.988$ & $\mathrm{R}^{2}=0.990$ & $\mathrm{R}^{2}=0.985$ \\
run 2 & $0.0648 \pm 0.0132$ & $0.0744 \pm 0.0158$ & $0.0661 \pm 0.0118$ \\
& $\mathrm{R}^{2}=0.988$ & $\mathrm{R}^{2}=0.985$ & $\mathrm{R}^{2}=0.991$ \\
\hline $\mathrm{k}_{\varnothing, \text { runs }}{ }^{a}\left[\mathrm{~min}^{-1}\right]$ & 0.064 & 0.068 & 0.063 \\
\hline \multicolumn{4}{c}{${ }^{a}$ average of two runs. }
\end{tabular}

\subsection{Photolysis of 1-B in ACN + Acid}

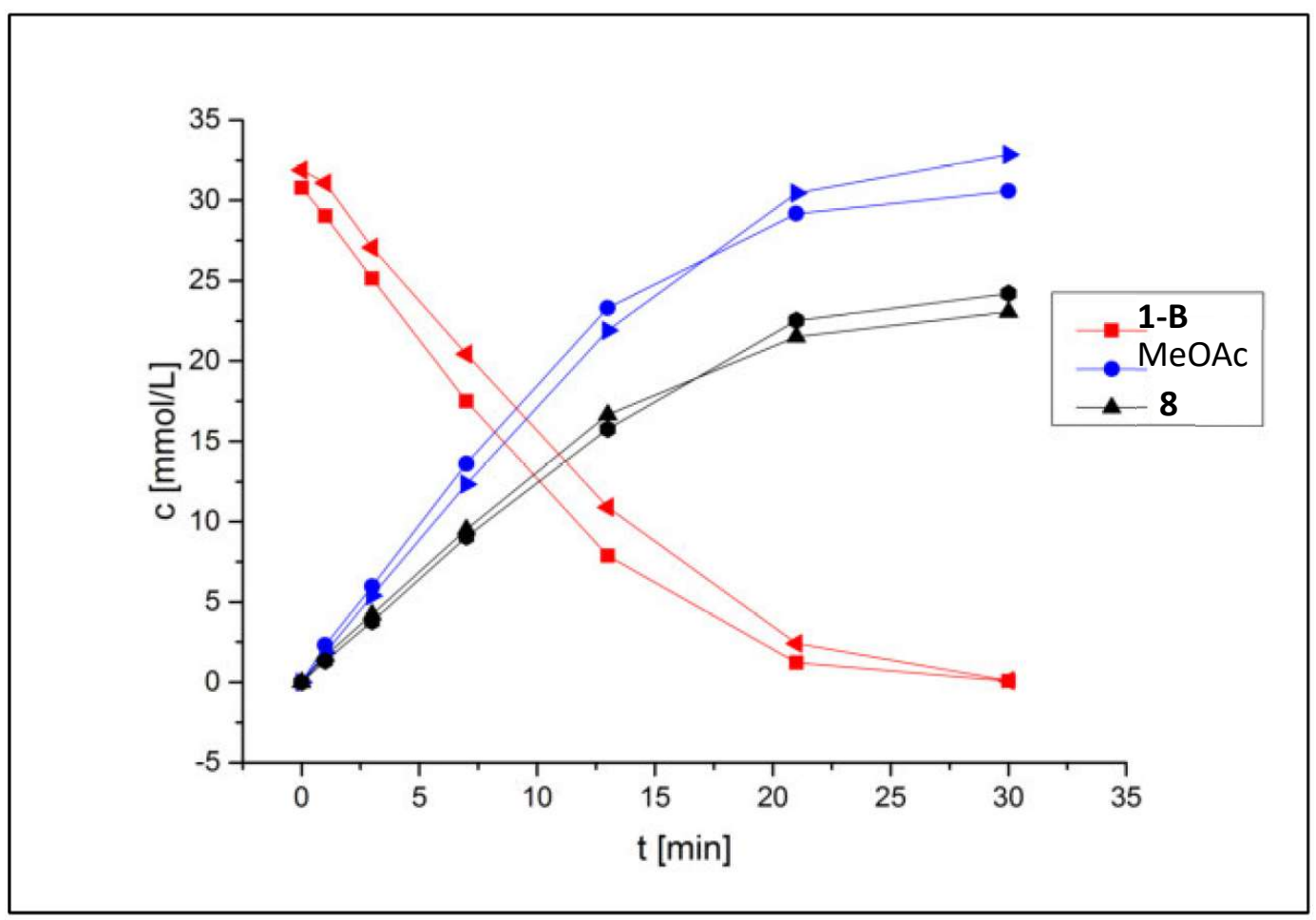

Figure S10. Kinetic traces obtained from ${ }^{1} \mathrm{H}$-monitoring of the photolysis of 1-B in $\mathrm{ACN}-\mathrm{d} 3$ in the presence of 2 equiv. of TFA. The photolysis was carried out in a quartz cuvette with UV-B light in two independent runs 
Table S28. Concentrations (c) of the reaction products determined by ${ }^{1} \mathrm{H}$ NMR monitoring of the photolysis of 1-B in quartz cuvettes (fluorescence cell, $1 \mathrm{~cm}$ path length) in presence of 2 equiv. of TFA in $\mathrm{ACN}-\mathrm{d}_{3}$ (degassed, kinetic traces see Figure $\mathrm{S7}$ ).

\begin{tabular}{c|cccccc}
\hline \multirow{2}{*}{$\mathrm{t}[\mathrm{min}]$} & \multicolumn{7}{c}{$\mathrm{c}[\mathrm{mmol} / \mathrm{L}]$} \\
& 1-B & 1-B & MeOAc & MeOAc & $\mathbf{8}$ & $\mathbf{8}$ \\
& run 1 & run 2 & run 1 & run 2 & run 1 & run 2 \\
\hline 0 & 30.76951 & 31.88847 & 0 & 0 & 0 & 0 \\
1 & 29.02488 & 31.07531 & 2.30771 & 1.82721 & 1.633860 & 1.32975 \\
3 & 25.14177 & 27.06055 & 5.96313 & 5.39553 & 4.22773 & 3.77878 \\
7 & 17.49555 & 20.43413 & 13.60936 & 12.33446 & 9.49855 & 9.04995 \\
13 & 7.87392 & 10.89310 & 23.30791 & 21.9010 & 16.65246 & 15.76566 \\
21 & 1.22463 & 2.41077 & 29.16950 & 30.45667 & 21.51712 & 22.51964 \\
30 & 0.08615 & 0.10842 & 30.57874 & 32.84193 & 23.04944 & 24.20335 \\
\hline max. & $0.3 \%$ & $0.3 \%$ & $99 \%$ & $103 \%$ & $75 \%$ & $76 \%$ \\
conv. & & & & & &
\end{tabular}

Table S29. Rate constants k (mono-exponential fit) of the different isomers of the bornyl oxadiazoline 1-B in the photolysis experiment in the quartz cuvette with UV-B light in presence of 2 equiv. of TFA in $\mathrm{ACN}-\mathrm{d} 3$.

\begin{tabular}{|c|c|c|c|c|c|c|}
\hline & \multicolumn{4}{|c|}{$\mathrm{k}\left[\mathrm{min}^{-1}\right]$ of the different diastereomrs A-D } & \multirow{2}{*}{$\begin{array}{c}\mathrm{k} \emptyset \text {,isomers }^{a} \\
{\left[\mathrm{~min}^{-1}\right]}\end{array}$} & \multirow{2}{*}{$\begin{array}{l}\mathrm{k} \phi, \text { total }^{b} \\
{\left[\mathrm{~min}^{-1}\right]}\end{array}$} \\
\hline & A-1-B & B-1-B & C-1-B & D-1-B & & \\
\hline run 1 & $\begin{array}{c}0.1006 \pm \\
0.0050 \\
R^{2}=0.999\end{array}$ & $\begin{array}{c}0.0808 \pm \\
0.0149 \\
R^{2}=0.987\end{array}$ & $\begin{array}{c}0.0843 \pm \\
0.0105 \\
R^{2}=0.994\end{array}$ & $\begin{array}{c}0.0646 \pm \\
0.0162 \\
R^{2}=0.982\end{array}$ & $\begin{array}{c}0.083 \\
\sigma=0.015\end{array}$ & $\begin{array}{c}0.0771 \pm \\
0.0126 \\
R^{2}=0.991\end{array}$ \\
\hline run 2 & $\begin{array}{c}0.0583 \pm \\
0.0124 \\
R^{2}=0.989\end{array}$ & $\begin{array}{c}0.0708 \pm \\
0.0124 \\
R^{2}=0.990\end{array}$ & $\begin{array}{c}0.0539 \pm \\
0.0127 \\
R^{2}=0.988\end{array}$ & $\begin{array}{c}0.0523 \pm \\
0.0134 \\
R^{\prime \prime}=0.987\end{array}$ & $\begin{array}{c}0.059 \\
\sigma=0.008\end{array}$ & $\begin{array}{c}0.0572 \pm \\
0.0117 \\
R^{2}=0.991\end{array}$ \\
\hline $\begin{array}{l}k_{\emptyset, \text { runs }}{ }^{c} \\
{\left[\mathrm{~min}^{-1}\right]}\end{array}$ & 0.079 & 0.076 & 0.069 & 0.058 & 0.071 & 0.067 \\
\hline
\end{tabular}


Table S30. Rate constants $k$ (mono-exponential fit) of the different reaction products of the photolysis (UVB) of 1-B in a quartz cuvette (fluorescence cell, $1 \mathrm{~cm}$ path length) in the presence of 2 equiv. TFA in ACN- $d_{3}$.

\begin{tabular}{c|cc}
\hline & \multicolumn{2}{|c}{$k\left[\mathrm{~min}^{-1}\right]$} \\
& MeOAc & $\mathbf{8}$ \\
\hline run 1 & $0.0807 \pm 0.0119$ & $0.0707 \pm 0.0104$ \\
& $\mathrm{R}^{2}=0.992$ & $\mathrm{R}^{2}=0.993$ \\
run 2 & $0.0588 \pm 0.0104$ & $0.0567 \pm 0.0109$ \\
& $\mathrm{R}^{2}=0.992$ & $\mathrm{R}^{2}=0.991$ \\
\hline $\mathrm{k}_{\varnothing, \text { runs }}{ }^{a}\left[\mathrm{~min}^{-1}\right]$ & 0.070 & 0.064 \\
\hline \multicolumn{3}{c}{${ }^{a}$ average of two runs. }
\end{tabular}

2.6 Photolysis of 1-F in ACN + Acid

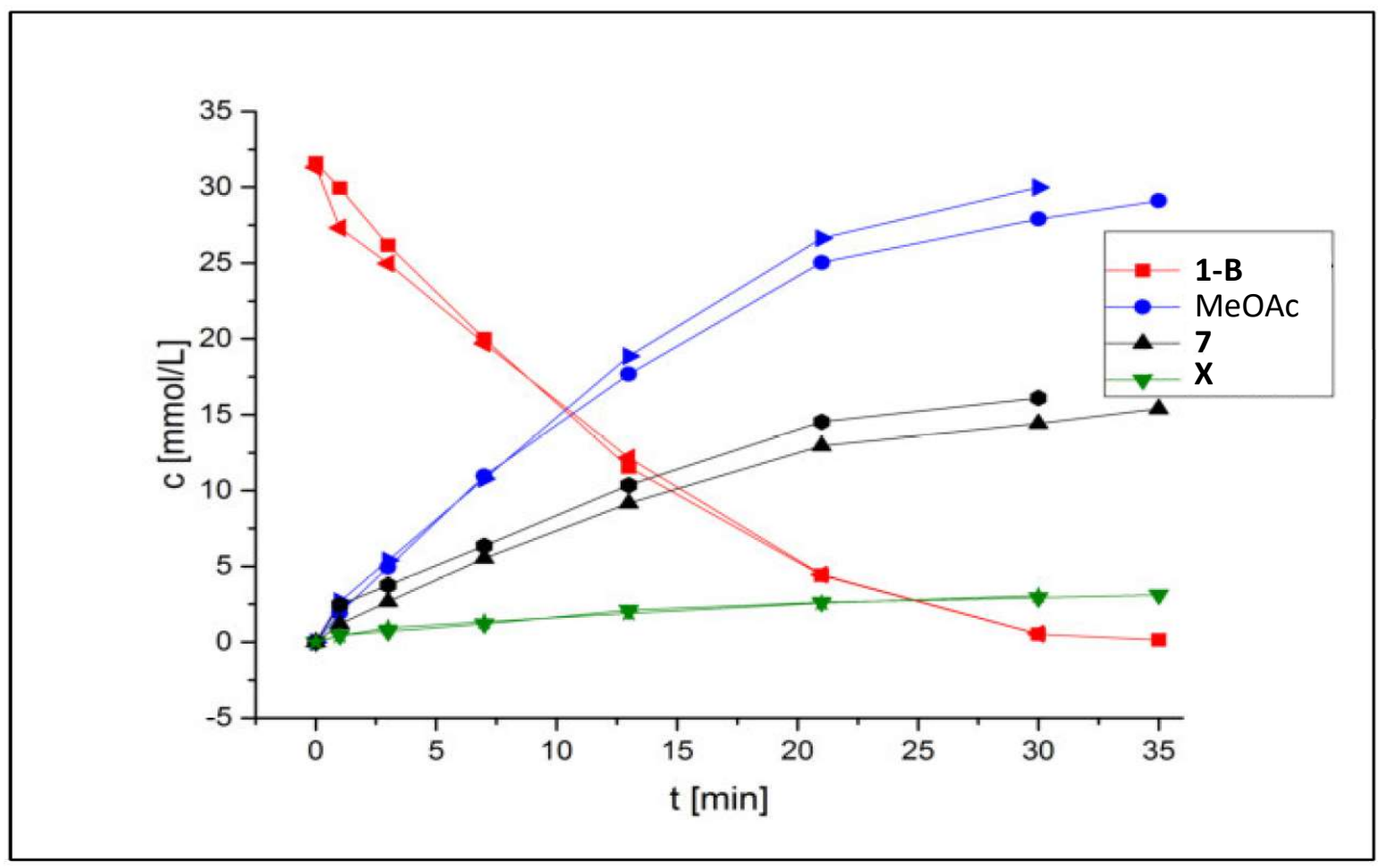

Figure S11. Kinetic trace of the photolysis of 1-F in ACN- $d_{3}$ in the presence of 2 equiv. of TFA. The photolysis was carried out in a quartz cuvette (fluorescence cell, $1 \mathrm{~cm}$ pathlength) with UV-B light in two independent runs. $X$ was a structurally unassigned compound. 
Table S31. Concentrations (c) of the reaction products determined by ${ }^{1} \mathrm{H}$ NMR of the photolysis of 1-F in quartz cuvettes (fluorescence cell, $1 \mathrm{~cm}$ path length) in presence of 2 equiv. of TFA in ACN- $d_{3}$ (plotted kinetic traces see Figure S8).

\begin{tabular}{c|cccccc}
\hline & \multicolumn{5}{|c}{$c[\mathrm{mmol} / \mathrm{L}]$} \\
$\begin{array}{c}\mathrm{t} \\
{[\mathrm{min}]}\end{array}$ & $\mathbf{1 - F}$ & $\mathbf{1 - F}$ & MeOAc & MeOAc & $\mathbf{7}$ & $\mathbf{7}$ \\
& run 1 & run 2 & run 1 & run 2 & run 1 & run 2 \\
\hline 0 & 31.60866 & 31.32898 & 0 & 0 & 0 & 0 \\
1 & 29.93972 & 27.322 & 1.94709 & 2.67549 & 1.19481 & 2.46246 \\
3 & 26.16565 & 24.97233 & 4.94043 & 5.38545 & 2.67409 & 3.75948 \\
7 & 20.01144 & 19.70906 & 10.95872 & 10.77404 & 5.53784 & 6.34412 \\
13 & 11.52452 & 12.17757 & 17.66924 & 18.85378 & 9.16967 & 10.34796 \\
21 & 4.39993 & 4.44245 & 25.03722 & 26.6265 & 12.96271 & 14.52098 \\
30 & 0.5089 & 0.54512 & 27.91045 & 29.99436 & 14.42303 & 16.10936 \\
35 & 0.1454 & - & 29.09893 & - & 15.39026 & - \\
\hline max. & $0.5 \%$ & $2 \%$ & $92 \%$ & $96 \%$ & $49 \%$ & $51 \%$ \\
conv. & $0.52 \%$ & & & & & \\
\hline
\end{tabular}

Table S32. Rate constants k (mono-exponential fit) of the different diastereomers A-D of 1-F in the photolysis experiment in a quartz cuvette (fluorescence cell, $1 \mathrm{~cm}$ pathlength) with UV-B light in presence of 2 equiv. of TFA in ACN- $d_{3}$ (degassed).

\begin{tabular}{|c|c|c|c|c|c|c|}
\hline & \multicolumn{4}{|c|}{$\mathrm{k}\left[\mathrm{min}^{-1}\right]$ of the diastereomers A-D } & \multirow{2}{*}{$\begin{array}{c}\mathrm{k} \emptyset, \text { isomers }^{a} \\
{\left[\mathrm{~min}^{-1}\right]}\end{array}$} & \multirow{2}{*}{$\begin{array}{l}\mathrm{k}_{\varnothing, \text { total }^{b}} \\
{\left[\mathrm{~min}^{-1}\right]}\end{array}$} \\
\hline & A-1-F & B-1-F & C-1-F & D-1-F & & \\
\hline run 1 & $\begin{array}{c}0.0552 \pm \\
0.0067 \\
R^{2}=0.996\end{array}$ & $\begin{array}{c}0.0605 \pm \\
0.0068 \\
R^{2}=0.996\end{array}$ & $\begin{array}{c}0.0684 \pm \\
0.0071 \\
R^{2}=0.996\end{array}$ & $\begin{array}{c}0.0635 \pm \\
0.0069 \\
R^{2}=0.996\end{array}$ & $\begin{array}{c}0.062 \\
\sigma=0.006\end{array}$ & $\begin{array}{c}0.0615 \pm \\
0.0057 \\
R^{2}=0.997\end{array}$ \\
\hline run 2 & $\begin{array}{c}0.0511 \pm \\
0.0150 \\
R^{2}=0.983\end{array}$ & $\begin{array}{c}0.0449 \pm \\
0.0094 \\
R^{2}=0.993\end{array}$ & $\begin{array}{c}0.0544 \pm \\
0.0114 \\
R^{2}=0.991\end{array}$ & $\begin{array}{c}0.0440 \pm \\
0.0117 \\
R^{\prime \prime}=0.989\end{array}$ & $\begin{array}{c}0.049 \\
\sigma=0.005\end{array}$ & $\begin{array}{c}0.0488 \pm \\
0.0091 \\
R^{2}=0.994\end{array}$ \\
\hline $\begin{array}{l}\mathrm{k} \emptyset \text {, runs }^{c} \\
{\left[\mathrm{~min}^{-1}\right]}\end{array}$ & 0.053 & 0.053 & 0.061 & 0.054 & 0.056 & 0.055 \\
\hline
\end{tabular}

${ }^{a}$ average of the independently determined rate constants of each isomer per run. ${ }^{b}$ fitting all data points of all runs and all isomers of the starting material together with a mono-exponential function. ${ }^{c}$ average of the independently determined rate constants of each run per isomer. 
Table S33. Rate constants $k$ (mono-exponential fit) of the different reaction products of the photolysis of 1-F in the photolysis experiment (UVB, quartz fluorescence cuvette, $1 \mathrm{~cm}$ pathlength) in the presence of 2 equiv. TFA in $A C N-d_{3}$.

\begin{tabular}{|c|c|c|}
\hline & \multicolumn{2}{|c|}{$\mathrm{k}\left[\mathrm{min}^{-1}\right]$} \\
\hline & MeOAc & 7 \\
\hline \multirow{2}{*}{ run 1} & $0.0591 \pm 0.0051$ & $0.0556 \pm 0.0051$ \\
\hline & $R^{2}=0.997$ & $R^{2}=0.997$ \\
\hline \multirow{2}{*}{ run 2} & $0.0495 \pm 0.0079$ & $0.0543 \pm 0.0125$ \\
\hline & $R^{2}=0.995$ & $R^{2}=0.989$ \\
\hline $\mathrm{k}_{\varnothing, \text { runs }}{ }^{a}$ & \multirow[b]{2}{*}{0.054} & \multirow[b]{2}{*}{0.055} \\
\hline$\left[\min ^{-1}\right]$ & & \\
\hline
\end{tabular}

${ }^{a}$ average of the independently determined rate constants of each run per isomer.

\subsection{Photolysis of 1-N in HFIP}

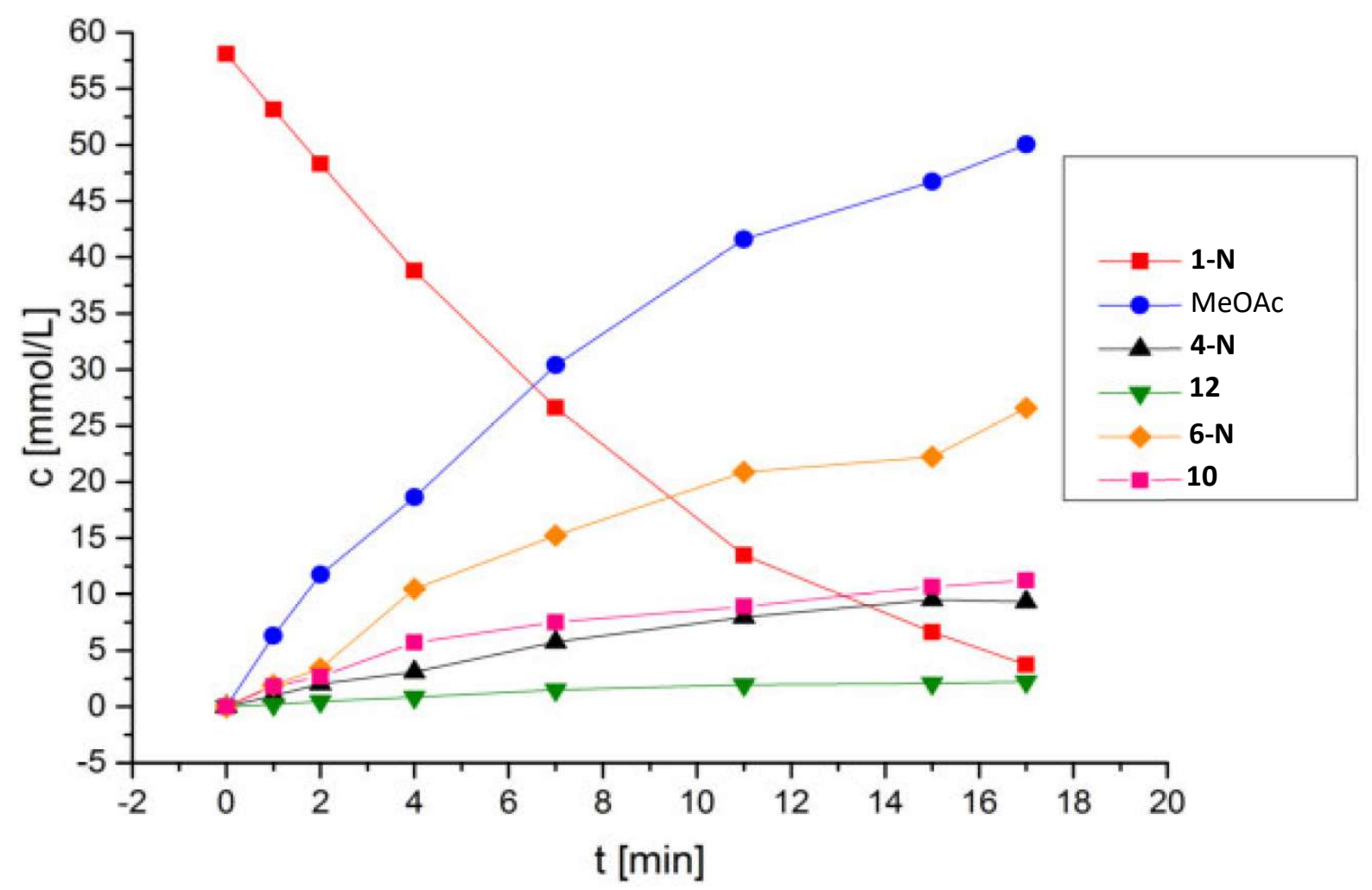

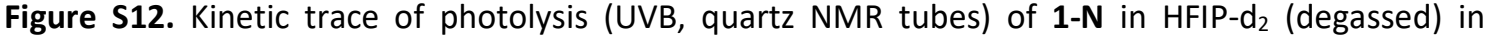
presence of 3 equiv. TMB. 
Table S34. Concentrations (c) of the reaction products determined by ${ }^{1} \mathrm{H}$ NMR of the photolysis of 1$\mathbf{N}$ in a quartz NMR tube in presence of 3 equiv. of TMB in HFIP-d2 (kinetic traces see Figure S9).

\begin{tabular}{ccccccc}
\hline \multirow{2}{*}{$\mathrm{t}[\mathrm{min}]$} & $\mathbf{6}$ & \multicolumn{6}{c}{$[\mathrm{mmol} / \mathrm{L}]$} \\
& $\mathbf{1 - N}$ & MeOAc & $\mathbf{4 - N}$ & $\mathbf{1 2}$ & $\mathbf{6 - N}$ & $\mathbf{1 0}$ \\
\hline 0 & 58.08921 & 0 & 0 & 0 & 0 & 0 \\
1 & 53.15163 & 6.3143 & 0.97067 & 0.19169 & 1.95180 & 1.81238 \\
2 & 48.29537 & 11.73983 & 2.01831 & 0.45310 & 3.38079 & 2.69244 \\
4 & 38.80359 & 18.61759 & 3.07786 & 0.83648 & 10.49091 & 5.71598 \\
7 & 26.62810 & 30.4097 & 5.74473 & 1.48127 & 15.23099 & 7.51094 \\
11 & 13.47670 & 41.59769 & 7.95909 & 1.95180 & 20.87726 & 8.89636 \\
15 & 6.64541 & 46.71535 & 9.52547 & 2.05636 & 22.23655 & 10.68261 \\
17 & 3.72933 & 50.02643 & 9.32361 & 2.23063 & 26.5934 & 11.24898 \\
\hline max. conv. & $6 \%$ & $86 \%$ & $16 \%$ & $4 \%$ & $46 \%$ & $19 \%$ \\
\hline
\end{tabular}

Table S35. Rate constants $\mathrm{k}$ (mono-exponential fit) of the different diastereomers of 1-N in the photolysis experiment (UVB) in quartz NMR tubes in presence of 3 equiv. of TMB in HFIP- $\mathrm{d}_{2}$.

\begin{tabular}{|c|c|c|c|c|c|c|}
\hline & \multicolumn{4}{|c|}{$\mathrm{k}\left[\mathrm{min}^{-1}\right]$ of the diastereomeres A-D } & \multirow{2}{*}{$\begin{array}{c}\varnothing \\
\mathrm{k}\left[\min ^{-1}\right] \\
a\end{array}$} & \multirow{2}{*}{$\begin{array}{c}\text { total } \\
\mathrm{k}\left[\min ^{-1}\right]^{b}\end{array}$} \\
\hline & A-1-N & B-1-N & C-1-N & D-1-N & & \\
\hline \multirow{3}{*}{ run 1} & $0.1014 \pm$ & $0.0921 \pm$ & $0.1156 \pm$ & $0.1156 \pm$ & 0.087 & $0.817 \pm$ \\
\hline & 0.0091 & 0.0087 & 0.0185 & 0.0253 & $\sigma=$ & 0.0076 \\
\hline & $R^{2}=0.998$ & $R^{2}=0.991$ & $R^{2}=0.992$ & $R^{2}=0.983$ & 0.015 & $R^{2}=0.998$ \\
\hline
\end{tabular}

${ }^{a}$ average of the independently determined rate constants of each run per isomer.

${ }^{b}$ fitting all data points of all runs and all isomers of the starting material together with a monoexponential function.

Table S36. Rate constants $k$ (mono-exponential fit) of the different reaction products of the photolysis of 1-N in the photolysis experiment in the quartz NMR tube with UV-B light in presence of 3 equiv. of TMB in HFIP- $\mathrm{d}_{2}$ (degassed).

\begin{tabular}{c|ccccc}
\hline \multirow{4}{*}{ run 1 } & MeOAC & $\mathbf{4 - N}$ & $\mathbf{k}\left[\mathrm{min}^{-1}\right]$ \\
& & & $\mathbf{1 2}$ & $\mathbf{6 - N}$ & $\mathbf{1 0}$ \\
\cline { 2 - 6 } & $0.0952 \pm 0.0091$ & $0.0837 \pm 0.0183$ & $0.1156 \pm 0.0185$ & $0.931 \pm 0.0253$ & $0.1361 \pm 0.0203$ \\
& $\mathrm{R}^{2}=0.998$ & $\mathrm{R}^{2}=0.991$ & $\mathrm{R}^{2}=0.992$ & $\mathrm{R}^{2}=0.983$ & $\mathrm{R}^{2}=0.991$ \\
\hline
\end{tabular}




\subsection{Photolysis of 1-B in HFIP}

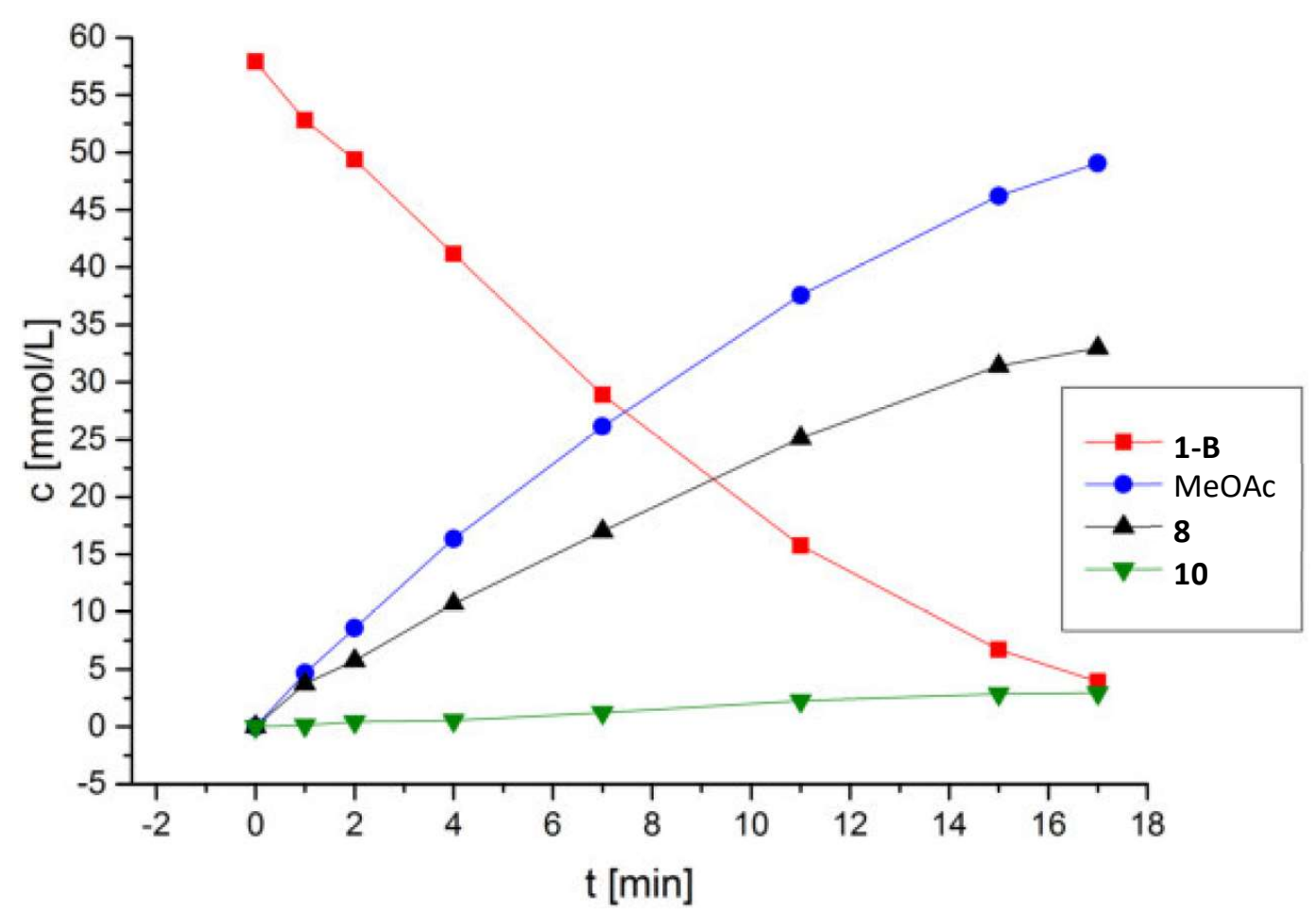

Figure S13. Kinetic traces of the photolysis (UVB) of 1-B in HFIP- $d_{2}$ (degassed) in the presence of 3 equiv. of TMB in a quartz NMR tube.

Table S37: Concentrations (c) in dependence of time determined by ${ }^{1} \mathrm{H}$ NMR of the photolysis (UVB) of 1-B in quartz NMR tubes in presence of 3 equiv. of TMB in HFIP- $d_{2}$ (degassed, plotted kinetic traces see Figure $\mathrm{S} 10)$.

\begin{tabular}{ccccc}
\hline \multirow{2}{*}[\mathrm{min}]{} & \multicolumn{4}{c}{$\mathrm{c}[\mathrm{mmol} / \mathrm{L}]$} \\
& $1-B$ & MeOAc & $\mathbf{8}$ & 10 \\
\hline 0 & 57.90267 & 0 & 0 & 0 \\
1 & 52.79566 & 4.65537 & 3.75209 & 0.13028 \\
2 & 49.39098 & 8.57539 & 5.76711 & 0.4169 \\
4 & 41.14564 & 16.3575 & 10.70041 & 0.52981 \\
7 & 28.92238 & 26.13727 & 17.02339 & 1.23333 \\
11 & 15.75532 & 37.56725 & 25.15292 & 2.2582 \\
15 & 6.7225 & 46.20633 & 31.42378 & 2.86618 \\
17 & 3.9258 & 49.06672 & 32.98715 & 2.93567 \\
\hline max. conv. & $7 \%$ & $85 \%$ & $57 \%$ & $5 \%$ \\
\hline
\end{tabular}


Table S38. Rate constants k (mono-exponential fit) of the different isomers of 1-B in the photolysis experiment (UVB) in quartz NMR tubes in presence of 3 equiv. of TMB in HFIP- $\mathrm{d}_{2}$ (degassed).

\begin{tabular}{|c|c|c|c|c|c|c|}
\hline & \multicolumn{4}{|c|}{$k\left[\mathrm{~min}^{-1}\right]$ of each diasteromer A-D } & \multirow{2}{*}{$\begin{array}{r}\mathrm{k}_{\varnothing, \text { isomers }}{ }^{a} \\
{\left[\mathrm{~min}^{-1}\right]}\end{array}$} & \multirow{2}{*}{$\begin{array}{l}\mathrm{k}_{\varnothing, \text { total }^{b}} \\
{\left[\mathrm{~min}^{-1}\right]^{b}}\end{array}$} \\
\hline & A-1-B & B-1-B & C-1-B & D-1-B & & \\
\hline \multirow{3}{*}{$\begin{array}{c}\text { run } \\
1\end{array}$} & $0.0626 \pm$ & $0.0803 \pm$ & $0.0538 \pm$ & $0.0459 \pm$ & \multirow{3}{*}{$\begin{array}{c}0.061 \\
\sigma=0.015\end{array}$} & $0.576 \pm$ \\
\hline & 0.0111 & 0.0077 & 0.0080 & 0.0082 & & 0.0078 \\
\hline & $R^{2}=0.996$ & $R^{2}=0.998$ & $R^{2}=0.998$ & $R^{2}=0.998$ & & $R^{2}=0.998$ \\
\hline
\end{tabular}

${ }^{a}$ average of the independently determined rate constants of each isomer per run. ${ }^{b}$ fitting all data points of all runs and all isomers of the starting material together with a mono-exponential function.

Table S39. Rate constants $k$ (mono-exponential fit) of the different reaction products of the photolysis (UVB) of 1-B in in quartz NMR tubes in presence of 3 equiv. of TMB in HFIP- $d_{2}$ (degassed).

\begin{tabular}{ccc}
\hline & \multicolumn{2}{c}{$k\left[\mathrm{~min}^{-1}\right]$} \\
& MeOAc & $\mathbf{8}$ \\
\hline run 1 & $0.0591 \pm 0.0030$ & $0.0507 \pm 0.0073$ \\
& $\mathrm{R}^{2}=1.000$ & $\mathrm{R}^{2}=0.998$ \\
\hline
\end{tabular}




\subsection{Photolysis of 1-F in HFIP}

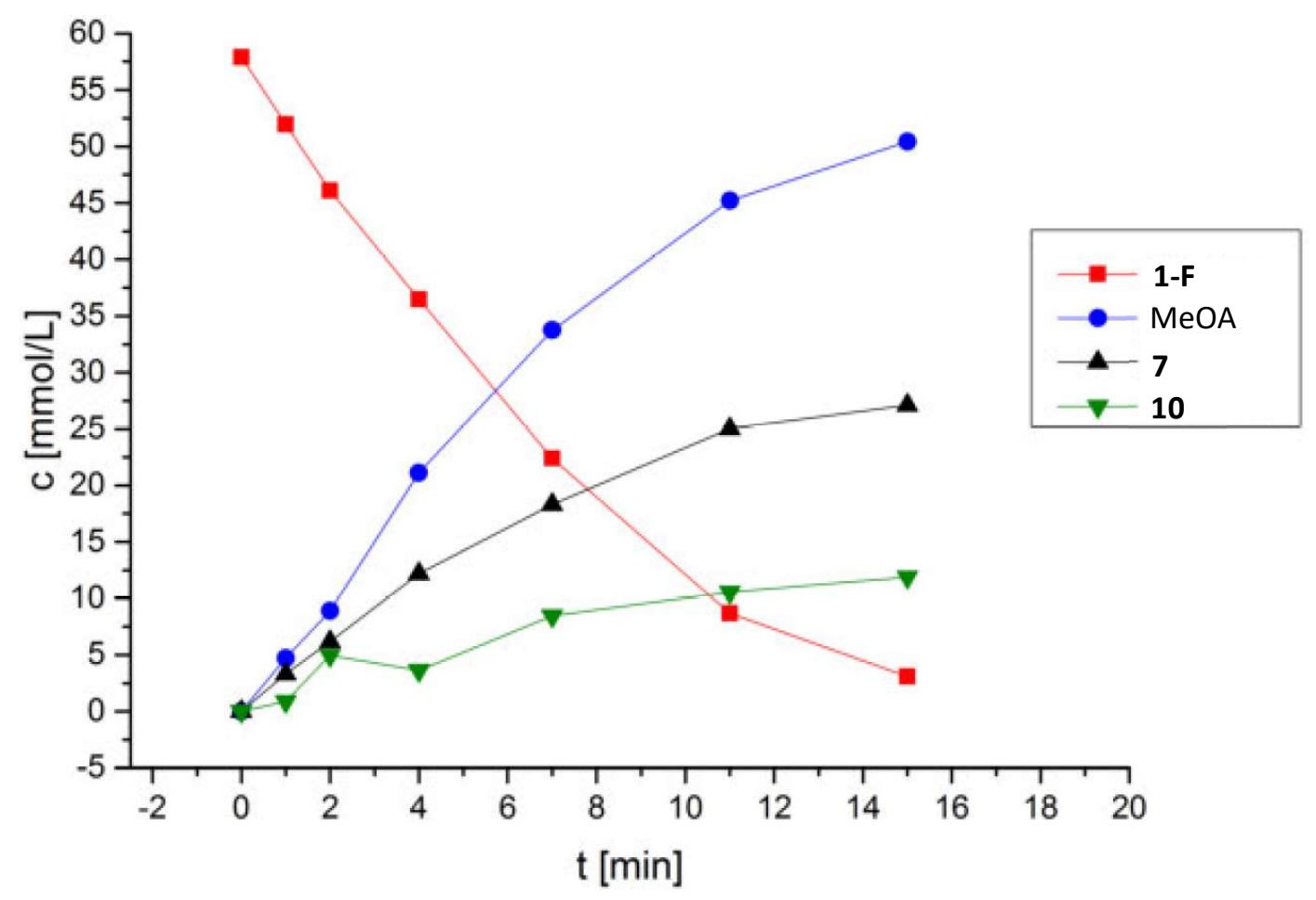

Figure S14. Kinetic traces of the photolysis (UVB) of 1-F in HFIP- $d_{2}$ in the presence of 3 equiv. of TMB in a quartz NMR tubes. The sample was degassed prior to irradiation.

Table S40: Concentrations (c) of substrate 1-F and reaction products determined by ${ }^{1} \mathrm{H}$ NMR of the photolysis of 1-F in a quartz NMR tube in presence of 3 equiv. of TMB in HFIP-d2 (plotted kinetic traces see Figure S11).

\begin{tabular}{c|cccc}
\hline \multirow{2}{*}{$\mathrm{t}[\mathrm{min}]$} & \multicolumn{4}{|c}{$\mathrm{c}[\mathrm{mmol} / \mathrm{L}]$} \\
\hline 0 & $\mathbf{1 - F}$ & MeOAc & $\mathbf{7}$ & $\mathbf{1 0}$ \\
1 & 57.90388 & 0 & 0 & 0 \\
2 & 51.96291 & 4.71338 & 3.31789 & 0.90330 \\
4 & 46.10307 & 8.88825 & 6.18413 & 4.96815 \\
7 & 36.47944 & 21.12334 & 12.19456 & 3.63057 \\
11 & 22.40880 & 33.74638 & 18.30921 & 8.45976 \\
15 & 8.69716 & 45.19977 & 25.01448 & 10.54430 \\
\hline max. conv. & 3.09207 & 50.44007 & 27.13376 & 11.89925 \\
\hline
\end{tabular}


Table S41. Rate constants $k$ (mono-exponential fit) of the different isomers of 1-F in the photolysis experiment in the quartz NMR tubes (UV-B light, 3 equiv. TMB in HFIP- $\mathrm{d}_{2}$ )

$$
\mathrm{k}\left[\mathrm{min}^{-1}\right]
$$

\begin{tabular}{ccccc} 
& 1-F & MeOAc & $\mathbf{8}$ & 10 \\
\hline run 1 & $0.1036 \pm 0.0151$ & $0.1015 \pm 0.0174$ & $0.1144 \pm 0.0131$ & $0.1068 \pm 0.0691$ \\
& $R^{2}=0.996$ & $R^{2}=0.994$ & $R^{2}=0.997$ & $R^{2}=0.913$ \\
& & & & \\
\hline
\end{tabular}

\section{TRIR studies \& TRUV}

\section{1 ns-TRUV}

The time-resolved UV-vis spectroscopy was run on a an Applied Photophysics LKS80 instrument with a SureliteTM SL I-10 Nd:YAG pump laser ( $355 \mathrm{~nm}$ third harmonic, $10 \mathrm{~Hz}$ repetition rate, $4-6 \mathrm{~ns}$ pulse width, $6 \mathrm{~mm}$ FWHM diameter, $26 \mathrm{~mJ}$ pulse energy, $266 \mathrm{~nm}$ fourth harmonic, $10 \mathrm{~Hz}$ repition rate, 4- 6ns pulse width, $6 \mathrm{~mm}$ FWHM diameter, $19 \mathrm{~mJ}$ pulse energy). The beam paths of pump and probe were set in an orthogonal alignment. The pump wavelength of the reported experiments was $266 \mathrm{~nm}$ and had been chosen based on the maximum absorption of the precursors $1 \mathbf{N}, 1 \mathbf{B}, \mathbf{1 F}$ (Figure S-12)

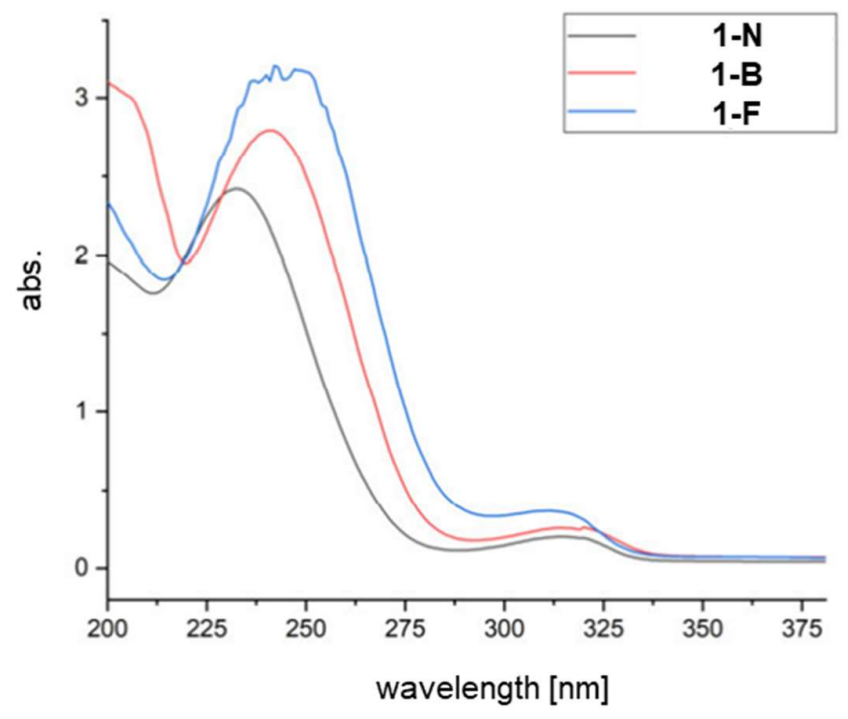

Figure S15 Absorption spectra of 1-N, 1-B, 1-F in HFIP (1.5mM). With the Nd:YAG and its $1^{\text {st }}$ to $4^{\text {th }}$ harmonics being available as a pump source the $266 \mathrm{~nm}$ wavelength was chosen.

In a set of experiments with 1,3,5-trimethoxybenzene (TMB) as trapping agent in HFIP as solvent a transient spectrum was recorded after pumping at $266 \mathrm{~nm}$ that matched reported data of the according cyclohexadienyl cation. ${ }^{23,24}$

${ }^{23}$ S. Steenken, M. Ashokkumar, P. Maruthamuthu, and R. A. McClelland J. Am. Chem. Soc. 1998, 120, 1192511931.

24 Pezacki, J. P.; Shukla, D.; Lusztyk, J.; Warkentin, J. Lifetimes of Dialkylcarbocations Derived from Alkanediazonium lons in Solution: Cyclohexadienyl Cations as Kinetic Probes for Cation Reactivity. J. Am. Chem. Soc. 1999, 121, 6589-6598. 
To countercheck if the UV signature might arise also from a photoprotonation of the TMB by HFIP during excitation (TMB has an absorption at $266 \mathrm{~nm}$ ) the experiment was re-run without the precursors 1 and the transient spectrum was recorded as well as the kinetic trace at its maximum absorption (details see Figure S13).
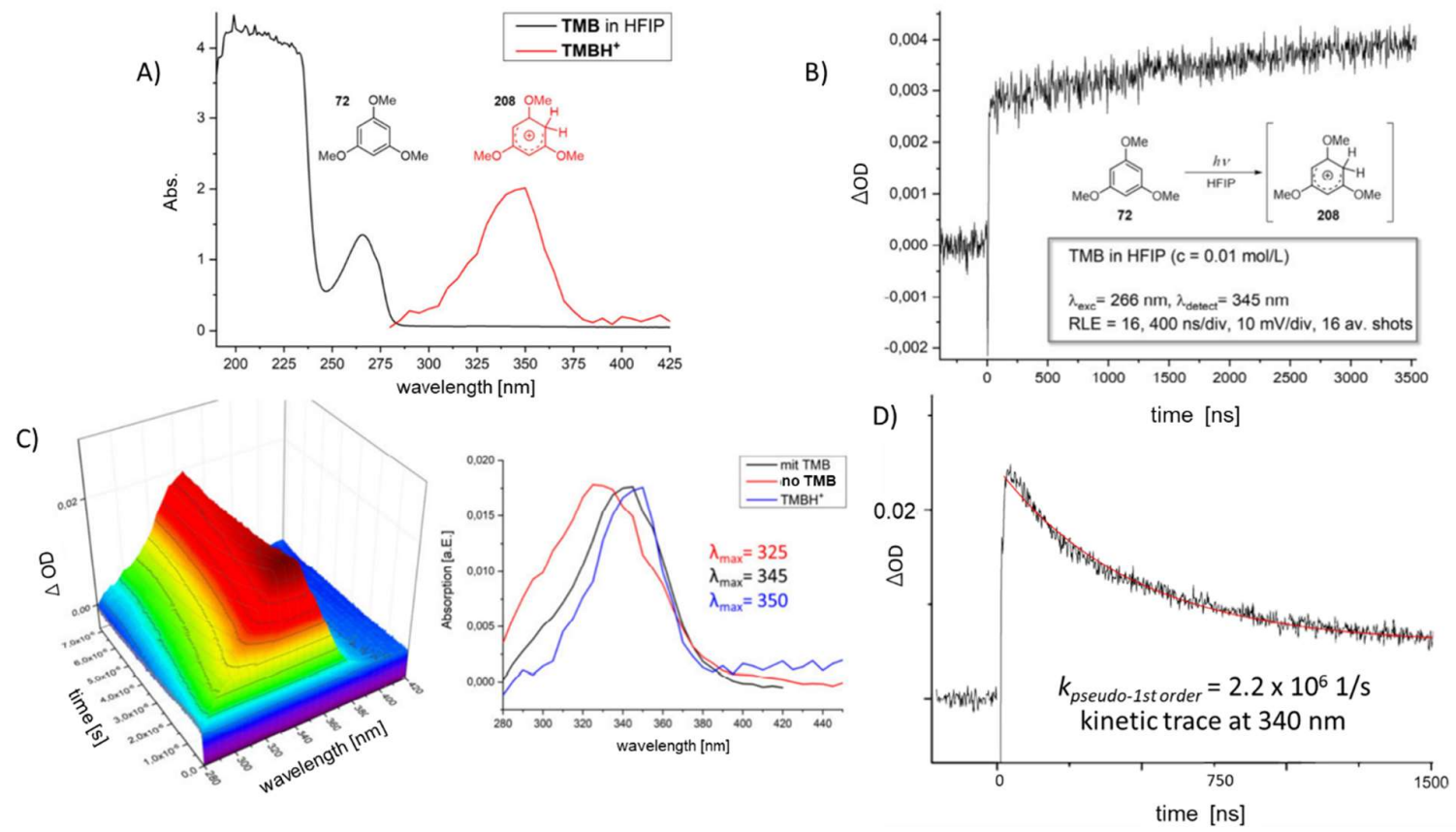

Figure S16 A) Comparison of the UV-vis data (back) of TMB in HFIP $(0.25 \mathrm{mM})$ and the transient absorption spectrum (red) of the $266 \mathrm{~nm}$ pump-probe experiment with a solution of TMB in HFIP (10 mM, RLE 16, averaged of 8 individual measurements). In comparison to published data ${ }^{23}$ this can be assigned to the photo protonation product $\mathbf{T M B H}^{+}$. B) Kinetic trace recorded at $345 \mathrm{~nm}$ of the same reaction solution as used in (A), i.e. $10 \mathrm{mM} \mathrm{TMB}$ in HFIP, $266 \mathrm{~nm}$ pump with RLE = 16 and 16 shots begin averaged using a resolution of $400 \mathrm{~ns} / \mathrm{div}$ and $10 \mathrm{mV} /$ div. C) left: $3 \mathrm{D}$ representation of the spectral evolution $(280 \mathrm{~nm}-420$ $\mathrm{nm}$ ) over time (up to $7 \mu \mathrm{s}$ ) measured of a HFIP solution of 1-B and 0.3 eq TMB under the same conditions used in (A). Right: comparison to the transient spectra recorded of the HFIP solutions of 1-B only (red), and TMB only (blue) and the mixture 1B + 0.3 eq TMB. D) Kinetic trace recorded of the experiment described in C (1-B, 0.3 eq TMB, HFIP).

One clearly sees that the transient spectra overlap of the different experiments (1-B, 1-B + TMB, TMB) in HFIP (Figure S13 Part C) but still imply the formation of a cyclohexadienyl cation by trapping one of the 1-B derived cations. This conclusion is drawn less on the only slightly differing absorption maxima ( $350 \mathrm{~nm}$ vs. $345 \mathrm{~nm}$ ) but mostly on the life-times of the species that differ quite significantly under otherwise same experimental conditions: $\mathrm{TMBH}^{+}\left(\lambda_{\max }=350 \mathrm{~nm}\right.$ ) with $\tau>3.5 \mu \mathrm{s}$ (no decline over the period of measurements) and the potential cyclohexadienyl cation $\left(\lambda_{\max }=345 \mathrm{~nm}\right)$ with a $\tau$ of $0.5 \mu \mathrm{s}$.

Puzzling was the transient spectrum arising from pumping a 1-B HFIP solution without TMB at $266 \mathrm{~nm}$. To elucidate what gave rise to the transient absorption at $325 \mathrm{~nm}$ that grows in with a rate constant of $\mathrm{k}_{\text {growth }}=5.8 \times 10^{6} 1 / \mathrm{s}$ and has an average lifetime of $5.3 \mu \mathrm{s}$ a couple of additional experiments were run, ie. TRUV in ACN, TRIR studies in HFIP and ACN (vide infra, section 3.2) and TDDFT calculations. The experiment $\left(\lambda_{\text {pump }}=266 \mathrm{~nm}\right)$ in HFIP without TMB indicated that the transient either comes from the precursor 1-B itself, i.e. an exited state or from intermediates of its follow-up chemistry. Based on the TRIR data (see below section 3.2) the determined life-time $(5.3 \mu \mathrm{s})$ of the transient is too long for a triplet excited state of the 1-B, since the fragmentation to the next intermediate 2-B is already 
happening on ps-scales. The similarity of the UV spectrum to the $\mathrm{TMBH}^{+}$and potential TMB-trapping product indicate however, that this transient may be a stabilized carbocation (Fig. S14). The TDDFT calculations of the carbocations evolving in the rearrangement process suggest that most likely the $\mathrm{Phe}^{+}$is the here observed cation. Repetition of this experiment in ACN was done to check on this hypothesis. ACN should avoid the protonation of the diazo 2-B and hence shut down the cationic pathway altogether. And indeed, no transient at the indicative $345 \mathrm{~nm}$ is arising, only a bleach of the parent was visible (see UV-spectra Fig. S12). Therefore, both transients at $325 \mathrm{~nm}$ and $345 \mathrm{~nm}$ produced under pumping the HFIP solutions with $266 \mathrm{~nm}$ most likely represent stabilized carbocations and hence are in line with the trapping studies of the stationary photolysis.
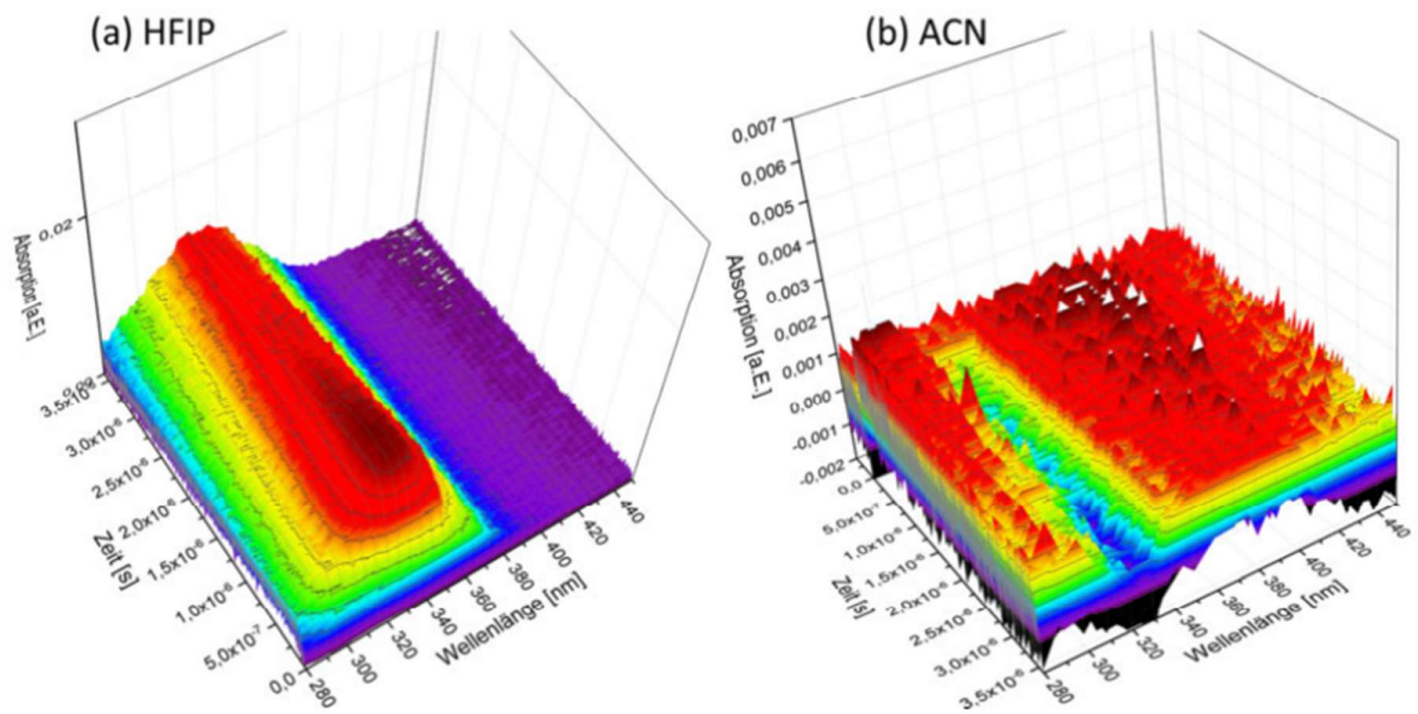

Figure S-17 The pump-probe experiments were done with a $42 \mathrm{mM}$ solution of 1-B in HFIP and ACN using as $\lambda_{\text {pump }}=266 \mathrm{~nm}$ with $0.3 \mathrm{~W} /$ pulse. The spectra were recorded from $280-450 \mathrm{nnm}$ with $4 \mathrm{~nm}$ resolution. The kinetic trace in HFIP was recorded at $330 \mathrm{~nm}$. a) shows the growing in and decline of a transient with a maximum at $325 \mathrm{~nm}$ whereas in ACN (B) this species is not evolving but only showing the bleach of the parent (1-B, compare Figure S12 for its UV-vis absorption spectrum).
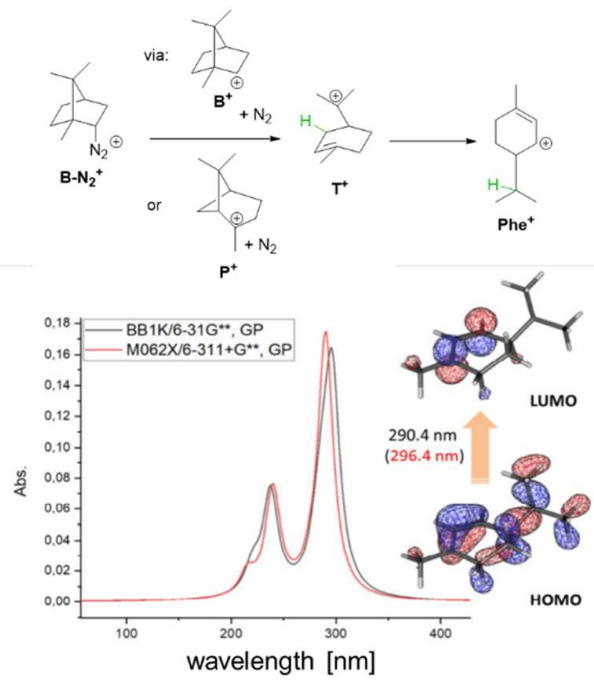

Figure S-18 TDDFT date on the postulated stabilized Cation $\mathrm{Phe}^{+}$. Also show the most likely pathway from $\mathbf{B}^{+}$to $\mathrm{Phe}^{+}$via $\mathbf{T}^{+}$.

Since the UV-spectra are helpful in the identification if the desired fragmentation and formation of cations occurs or not, they do not reveal enough structural information to tell apart the different carbo 
cationic structures with high enough certainty. To obtain an unambiguous interpretation of the intermediates and products, we chose the product analysis of the stationary photolysis by NMR to complement the ns-TRUV by TMB trapping.

\section{2 fs-TRIR}

Femtosecond transient infrared absorption spectroscopy (fs-TRIR) was performed by exciting the sample with 267-nm pulses derived from a two-step co-linear third-harmonic generation(400-nm pulses from second harmonic generation of $800-\mathrm{nm}$ pulses, $267-\mathrm{nm}$ pulses from sum-frequency generation of $800-\mathrm{nm}$ and $400-\mathrm{nm}$ pulses). The excitation pulses had a pulse duration of $100 \mathrm{fs}$, a pulse energy of $4.5 \mu \mathrm{J}$ and a focal size of $170 \mu \mathrm{m}$ in the sample plane.

Probe pulses were generated in a three-step process in a home-built optical parametric amplifier, pumped by $200-\mu \mathrm{J}$ pulses from a commercial Ti:sapphire laser amplifier (Spectra Physics Spitfire Ace, $90 \mathrm{fs}, 800 \mathrm{~nm}, 11 \mathrm{~nm}$ bandwidth $3 \mathrm{kHz}$ repetition rate). Dual-stage signal and idler amplification from a sapphire-generated white-light continuum in BBO and subsequent difference frequency generation in AgGaS2 yielded pulses at $4.93 \mu \mathrm{m}\left(2030 \mathrm{~cm}^{-1}\right)$ and $5.78 \mathrm{um}\left(1730 \mathrm{~cm}^{-1}\right)$ wavelength of $\sim 100 \mathrm{fs}$ duration with a FWHM bandwidth of $275 \mathrm{~cm}^{-1}$ and $260 \mathrm{~cm}^{-1}$, respectively. Absorbance changes were measured by imaging the transmitted probe pulses in a Triax180 spectrometer onto a $32 \times 2 \mathrm{MCT}$ detector array (Infrared Systems).

The sample (NORCOX in ACN and HFIP solution of $180 \mathrm{mM}$ ) was measured between 1-mm thick $\mathrm{CaF}_{2}$ windows in a commercial flow cell (Harrick Scientific Products) and continuously cycled in a closedloop liquid circuit by a peristaltic pump (Ismatec Reglo Digital). Sample consumption by the irreversible excitation process was monitored by pump-probe signal strength and the sample replaced after $\sim 1 / 3$ sample consumption.

Product formation was monitored by the induced absorption of the stretching vibration of the diazo group of the diazonium ion and the keto group of the split-off ester. The absorbance changes were analyzed by subtracting a polynomial fit to the background which varying slowly with frequency. The resulting absorption lineshapes were analyzed by a sum of two Gaussians (diazo stretching) and a Lorentzian (CO stretching).

The resulting induced absorption lineshapes, indicative of the diazonium ion and the ester, respectively, are displayed in Fig. 1 (panel A) for a time delay of 60 ps after 267-nm excitation. The instrument response function was $\sim 180 \mathrm{fs}$ as measured in $0.5-\mathrm{mm}$ thick ZnSe. The amplitude of the fit lineshapes is displayed in panels B (keto stretching) and C (diazo stretching) of the same figure. It is evident that the absorption of both products in either ACN or HFIP solution grows in on two timescales describes by time constants in Fig. 1. We attribute the picosecond time constant to the formation of the products and the longer time constant to product thermalization. We note that the lineshape formation is complex and is governed by anharmonic coupling to other modes that are transiently populated during the intramolecular vibrational energy redistribution process and subsequent thermalization with the solvent. A more detailed analysis of the product formation and thermalization process, including a differentiated lineshape analysis will be published in a subsequent manuscript. 


\section{Computational Chemistry}

\subsection{General Information}

Geometry optimizations of stationary points were performed using different levels of DFT such as $B 3 L Y P^{25}$ and M062X in conjunction with the $6-31 G^{*}, 6-31+G(d, p)$ or $6-311+G^{* *}$ basis sets ${ }^{26}$. Stationary points were identified as TSs or minima by frequency calculations (one or no imaginary frequency respectively). In order to verify that the transition states correspond to the expected reaction pathways, intrinsic reaction coordinate (IRC) calculations were conducted for all TS structures. B3LYP is most widely used in predicting geometries and chemical behaviour of monoterpenes, terpenoid carbocations and other small biologically active molecules. ${ }^{27}$ In addition, the functional was also previously applied successfully in the investigation of $\Delta^{3}-1,3,4$-oxadiazoline reaction mechanisms. ${ }^{2,28}$

In order to evaluate possible shortcomings in the results from B3LYP calculations arising from the neglect of dispersion effects the Truhlar functional M06-2X ${ }^{29}$ was employed.

BOMD trajectory calculations for the dissociation of diazonium ions $\mathbf{3}$ and the corresponding potential energy surface (PES) scans were executed at the M062X level of theory. The BOMD algorithm was used as implemented in used version of Gaussian09.

TDDFT calculations were used to compare experimental with computed data of potential transients.

All calculations presented herein were performed with the Gaussian09 program package. ${ }^{30}$

Stationary points were identified as TSs or minima by harmonic frequency calculations. In order to verify that the transition states correspond to the expected reaction pathways, intrinsic reaction coordinate (IRC) calculations were conducted for all TS structures. To identify the structural area of reaction path bifurcations BOMD simulations were used in combination with projected frequency analysis on along the IRC of the diazonium TS structures at the B3LYP/6-311+G** level of theory ${ }^{31}$.

${ }^{25}$ B3: Becke, A. D. J. Chem. Phys. 1993, 98, 5648; LYP: Lee, C.; Yang, W.; Parr, R. G. Phys. Rev. B 1988, $37,785$.

${ }^{26}$ 6-31G: [a] Ditchfield, R; Hehre, W. J.; Pople, J. A. J. Chem. Phys. 1971, 54, 724; 6-311: [b] Raghavachari, K.; Binkley, J. S.; Seeger, R.; Pople J. A. J. Chem. Phys. 1980, 72, 650-54. [c] McLean, A. D.; Chandler, G. S. J. Chem. Phys. 1980, 72, 5639-48.

27 [a] Hong, Y., Tantillo, D. Biosynthetic consequences of multiple sequential post-transition-state bifurcations. Nature Chem 6, 104-111 (2014). [b] Y. J. Hong, D. J. Tantillo, 'Quantum chemical dissection of the classic terpinyl/pinyl/bornyl/camphyl cation conundrum-the role of pyrophosphate in manipulating pathways to monoterpenes', Org. Biomol. Chem. 2010, 8, $4589-4600$.

${ }^{28}$ Mieusset, Jean-Luc; Billing, Peter; Abraham, Michael; Arion, Vladimir B.; Brecker, Lothar; Brinker, Udo H. '2Methoxy- $\triangle 3$-1,3,4-oxadiazoline, a Multipurpose Precursor for the Generation of a Carbene, an Ylide, or a Diazo Compound', Eur. J. Org. Chem. 2008, 31, 5336-5345.

${ }^{29}$ Zhao, Y., Truhlar, D.G Theor Chem Account 2008, 120, 215-241

30 Gaussian 09, Revision E.01, M. J. Frisch, G. W. Trucks, H. B. Schlegel, G. E. Scuseria, M. A. Robb, J. R. Cheeseman, G. Scalmani, V. Barone, B. Mennucci, G. A. Petersson, H. Nakatsuji, M. Caricato, X. Li, H. P. Hratchian, A. F. Izmaylov, J. Bloino, G. Zheng, J. L. Sonnenberg, M. Hada, M. Ehara, K. Toyota, R. Fukuda, J. Hasegawa, M. Ishida, T. Nakajima, Y. Honda, O. Kitao, H. Nakai, T. Vreven, J. A. Montgomery, Jr., J. E. Peralta, F. Ogliaro, M. Bearpark, J. J. Heyd, E. Brothers, K. N. Kudin, V. N. Staroverov, T. Keith, R. Kobayashi, J. Normand, K. Raghavachari, A. Rendell, J. C. Burant, S. S. Iyengar, J. Tomasi, M. Cossi, N. Rega, J. M. Millam, M. Klene, J. E. Knox, J. B. Cross, V. Bakken, C. Adamo, J. Jaramillo, R. Gomperts, R. E. Stratmann, O. Yazyev, A. J. Austin, R. Cammi, C. Pomelli, J. W. Ochterski, R. L. Martin, K. Morokuma, V. G. Zakrzewski, G. A. Voth, P. Salvador, J. J. Dannenberg, S. Dapprich, A. D. Daniels, O. Farkas, J. B. Foresman, J. V. Ortiz, J. Cioslowski, and D. J. Fox, Gaussian, Inc., Wallingford CT, 2013.

${ }^{31}$ S. Maeda, Y. Harabuchi, Y. Ono, T. Taketsugu, K. Morokuma, 'Intrinsic Reaction Coordinate: Calculation, Bifurcation, and Automated Search', Int. J. Qua. Chem. 2015, 115, 258 - 269. 
BOMD trajectory calculations for the dissociation of diazonium ions $\mathbf{F}-\mathbf{N}_{2}{ }^{+}$and $\mathbf{B}-\mathbf{N}_{2}{ }^{+}$and the corresponding potential energy surface (PES) scans were executed at the M06-2X/6-31G* level of theory.

\subsection{Optimized structures}

4.2.1 Structures optimized at the M06-2X/6-311+G** level of theory

\begin{tabular}{|l|l|l|}
\hline Bornyl cation $\left(\mathbf{B}^{+}\right)$, TS & \\
M06-2X/6-311+G** &
\end{tabular}

\section{optimized xyz-matrix}

\begin{tabular}{|c|c|c|c|}
\hline & 1.38653400 & -0.52266200 & -1.26348700 \\
\hline & 2.34380900 & -0.03073800 & -1.49055000 \\
\hline & 1.32974500 & -1.37964300 & -1.9400690 \\
\hline & 0.25277800 & 0.39474000 & -1.51658800 \\
\hline & -0.15723700 & 0.55253900 & $-2.5159260 c$ \\
\hline & -0.36725400 & 0.87243300 & -0.34705300 \\
\hline & -1.67870000 & 1.61207100 & -0.44743600 \\
\hline 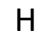 & -2.36118900 & 1.13841200 & -1.1554860 \\
\hline & -2.16380600 & 1.65289600 & 0.5292990 \\
\hline & -1.50926400 & 2.64203800 & $-0.7698870 c$ \\
\hline & 0.69857200 & 1.46910800 & 0.63842500 \\
\hline & 1.20988200 & 2.29455200 & 0.1425490 \\
\hline & 0.14838200 & 1.89177800 & $1.4791370 \mathrm{C}$ \\
\hline & 1.65237600 & 0.32726400 & 1.05005100 \\
\hline & 2.69410700 & 0.56067900 & 0.8314580 \\
\hline $\mathrm{H}$ & 1.58525600 & 0.11690500 & 2.1169480 \\
\hline C & 1.15477300 & -0.85807000 & 0.2187270 \\
\hline & 1.55329700 & -1.82519100 & 0.5236250 \\
\hline & -0.39159800 & -0.75105200 & 0.2862680 \\
\hline & -1.17547500 & -1.76060000 & $-0.550106 c$ \\
\hline $\mathrm{H}$ & -0.76647800 & -1.95165600 & -1.542613 \\
\hline-1 & -1.19575400 & -2.71169200 & -0.012835 \\
\hline & -2.21128100 & -1.43433500 & -0.665498 \\
\hline & -1.02209500 & -0.68263500 & 1.6665020 \\
\hline & -0.63729300 & 0.10594200 & 2.308425 \\
\hline & -2.10616300 & -0.58401700 & 1.594566 \\
\hline & -0.81548000 & -1.64205400 & 2.155033 \\
\hline
\end{tabular}

\section{Thermodynamic Data}

Zero-point correction=

0.247060 (Hartree/Particle)

Thermal correction to Energy=

0.256896

Thermal correction to Enthalpy=

0.257840

Thermal correction to Gibbs Free Energy=

Sum of electronic and zero-point Energies=

0.213497

Sum of electronic and thermal Energies=

$-390.656779$

Sum of electronic and thermal Enthalpies=

$-390.646943$

$-390.645999$ 
Sum of electronic and thermal Free Energies $=\quad-390.690341$

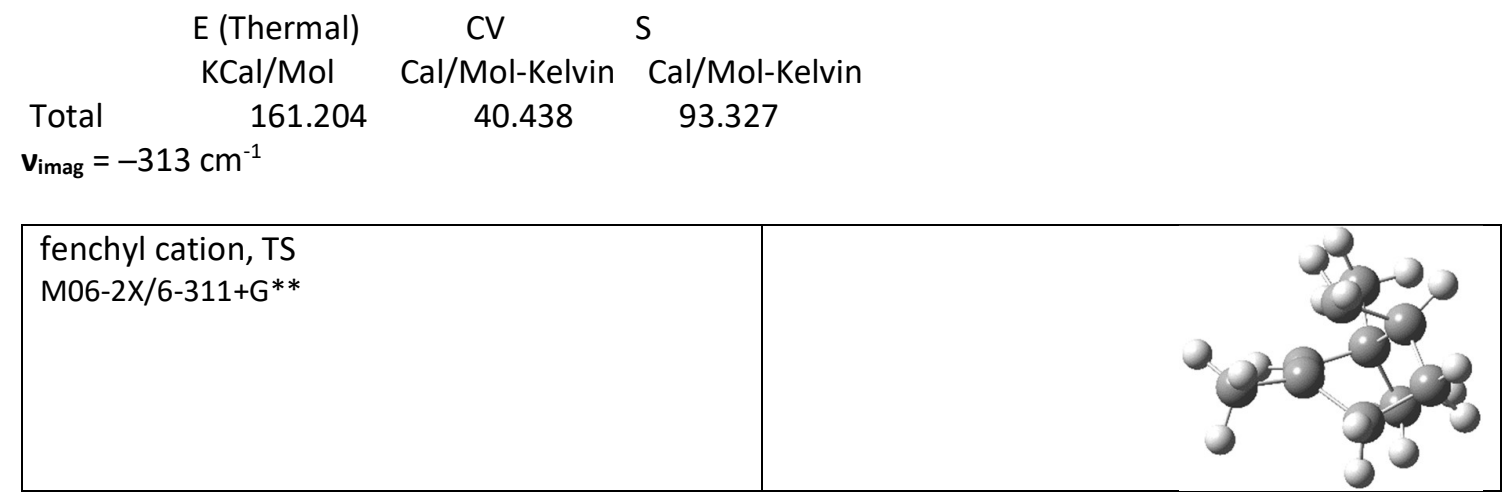

\section{optimized xyz-matrix}

$\mathrm{H}$

0.62580600

1.33754900

$\begin{array}{lll}0.32505900 & -1.25981800 & 1.76183100\end{array}$

C $\quad 0.00294300 \quad-0.92679100-0.59038900$

H $\quad-0.06955800 \quad-1.91059900 \quad-1.06463700$

C $\quad \begin{array}{llll}\text { C } & 1.24546600 & -0.35776500 & -0.20450500\end{array}$

C $\quad 2.51535100-1.14269800-0.43908700$

H $\quad 2.37771000 \quad-2.20774000 \quad-0.24578700$

H $\quad 2.85226300-1.01581800-1.47007400$

$\begin{array}{llll}\mathrm{H} & 3.30714000 & -0.77380900 & 0.21498500\end{array}$

$\begin{array}{llll}\text { C } & 1.32841500 & 1.18208300 & -0.45974500\end{array}$

H $\quad 2.32643300 \quad 1.49338000 \quad-0.14804700$

H $\quad 1.24474300 \quad 1.37383100-1.53008000$

$\begin{array}{llll}\text { C } & 0.21861100 & 1.85066500 & 0.37931500\end{array}$

$\begin{array}{llll}\mathrm{H} & 0.64948200 & 2.46236500 & 1.17222600\end{array}$

H $\quad-0.42988000 \quad 2.49580900 \quad-0.21132600$

$\begin{array}{lllll}\text { C } & & -0.52336700 & 0.65943200 & 0.98750600\end{array}$

H $\quad \begin{array}{llll}\text { H } & -1.19391700 & 0.91181300 & 1.80753200\end{array}$

C $\quad-1.20338500 \quad-0.20593900-0.11125500$

$\begin{array}{lllll}\text { C } & -2.21315200 & -1.20072700 & 0.49468100\end{array}$

$\mathrm{H} \quad-3.08676800-0.63845700 \quad 0.82992000$

$\begin{array}{lllll}\mathrm{H} & -2.54064300 & -1.92800400 & -0.25095100\end{array}$

H $\quad-1.80886000 \quad-1.73894500 \quad 1.35314800$

$\begin{array}{lllll}\text { C } & & -1.87141200 & 0.52761500 & -1.29522600\end{array}$

$\mathrm{H} \quad \begin{array}{lllll}\mathrm{C} & -1.17991100 & 1.16871000 & -1.84185100\end{array}$

$\mathrm{H} \quad-2.31007300-0.18819600 \quad-1.99259000$

H $\quad \begin{array}{llll}-2.67711600 & 1.14781400 & -0.89576500\end{array}$

\section{thermodynamic data}

Zero-point correction=

Thermal correction to Energy=

Thermal correction to Enthalpy=

Thermal correction to Gibbs Free Energy=

Sum of electronic and zero-point Energies=

Sum of electronic and thermal Energies=

Sum of electronic and thermal Enthalpies=

Sum of electronic and thermal Free Energies=
0.247405 (Hartree/Particle)
0.257216

0.258160

0.213780

$-390.655825$

$-390.646014$

$-390.645069$

$-390.689450$ 


$\begin{array}{cccc} & \text { E (Thermal) } & \text { CV } & \text { S } \\ \text { KCal/Mol } & \text { Cal/Mol-Kelvin } & \text { Cal/Mol-Kelvin } \\ \text { Total } & 161.406 & 40.233 & 93.406 \\ & & & \\ \text { Vimag }_{\text {ing }}-356 \mathrm{~cm}^{-1} & & \end{array}$

\begin{tabular}{|l|l|}
\hline Pinyl cation \\
M06-2X/6-311+G**
\end{tabular}

optimized xyz-matrix

$\begin{array}{llll}\text { C } & 1.86046300 & 0.24565200 & -0.89103800 \\ H & 2.87655300 & 0.04713900 & -0.54469000\end{array}$

H $\quad 1.86526200 \quad 0.88637800 \quad-1.77199500$

$\begin{array}{lllll}\text { C } & -0.25974800 & 0.01170100 & -0.86885700\end{array}$

$\begin{array}{llll}\mathrm{H} & -0.29488500 & 0.76923600 & -1.65323700\end{array}$

$\begin{array}{llll}\text { C } & 0.89390200 & 0.73437700 & 0.20928900\end{array}$

$\begin{array}{llll}\text { C } & 0.71610400 & 2.16799800 & 0.59622100\end{array}$

H $\quad 0.55442600 \quad 2.80995200 \quad-0.27143300$

$\begin{array}{llll}\mathrm{H} & -0.10041700 & 2.30177000 & 1.30964000\end{array}$

$\begin{array}{llll}\mathrm{H} & 1.63778800 & 2.49991400 & 1.08675000\end{array}$

$\begin{array}{llll}\text { C } & 1.04975900 & -0.34591400 & 1.27208200\end{array}$

H $\quad \begin{array}{llll}\text { H } & 1.97697100 & -0.15942000 & 1.82029900\end{array}$

H $\quad 0.24452800 \quad-0.35264000 \quad 2.00903300$

$\begin{array}{llll}\text { C } & 1.13028100 & -1.61593500 & 0.38102800\end{array}$

H $\quad 2.11650300 \quad-2.07872800 \quad 0.42182000$

$\begin{array}{llll}\mathrm{H} & 0.40735200 & -2.38326700 & 0.64636600\end{array}$

C $\quad 0.91709900 \quad-0.97605600-1.00505300$

H $\quad 0.95098700 \quad-1.62409600 \quad-1.87761100$

C $\quad-1.52057700 \quad-0.13807300 \quad-0.25311300$

$\begin{array}{lllll}\text { C } & -1.97942100 & -1.35918100 & 0.43738300\end{array}$

H $\quad-3.06420100 \quad-1.36563300 \quad 0.54394700$

H $\quad-1.62792000-2.26465500 \quad-0.05673900$

H $\quad-1.55835200-1.35771800 \quad 1.45405600$

$\begin{array}{lllll}\text { C } & -2.44495200 & 1.01415500 & -0.26121800\end{array}$

$\mathrm{H} \quad \begin{array}{llll}\mathrm{C} & -1.98802300 & 1.93496600 & -0.61862000\end{array}$

H $\quad-3.28116500 \quad 0.75169700 \quad-0.92636200$

H $\quad-2.89286600 \quad 1.15276000 \quad 0.72842800$

\section{thermodynamic data}

Zero-point correction=

Thermal correction to Energy=

Thermal correction to Enthalpy=

Thermal correction to Gibbs Free Energy=

Sum of electronic and zero-point Energies=

Sum of electronic and thermal Energies=

Sum of electronic and thermal Enthalpies=

Sum of electronic and thermal Free Energies=
0.246680 (Hartree/Particle)

$$
0.257274
$$

0.258218

0.211858

$-390.650061$

$-390.639467$

$-390.638523$

$-390.684883$ 


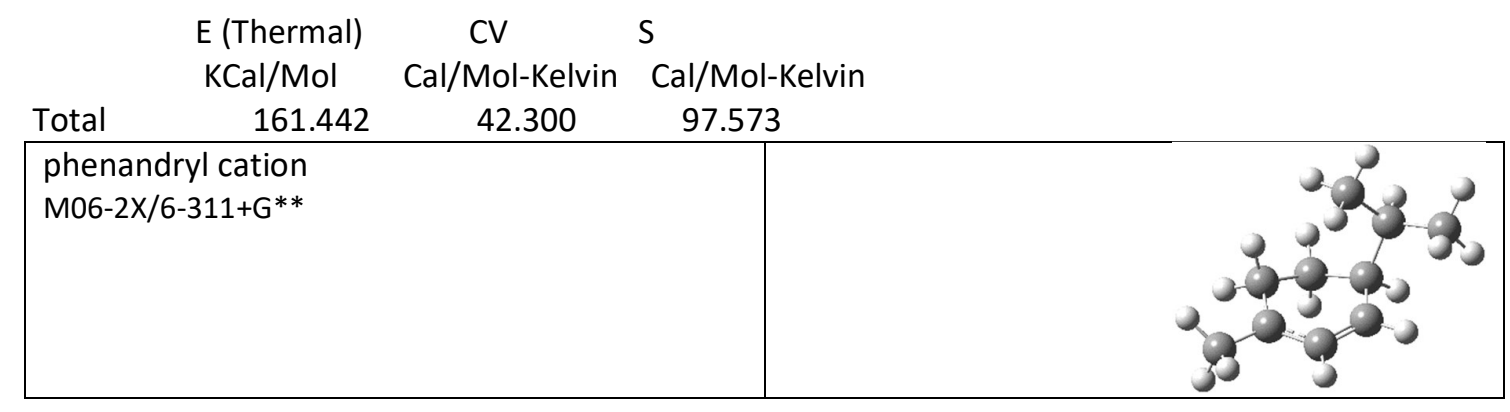

\begin{tabular}{|c|c|c|c|}
\hline \multicolumn{4}{|c|}{ optimized xyz-matrix } \\
\hline C & 1.35752400 & -1.14726300 & -0.19203400 \\
\hline C & 0.18752000 & -1.14675800 & -1.17575400 \\
\hline C & -0.81783600 & -0.01428200 & -0.93329700 \\
\hline C & -0.15593500 & 1.23808500 & -0.52877500 \\
\hline C & 1.14198700 & 1.34714100 & -0.09072600 \\
\hline C & 1.90387500 & 0.19427800 & 0.11635700 \\
\hline C & 3.26176400 & 0.28177600 & 0.68526800 \\
\hline C & -1.99441200 & -0.36488500 & 0.04891800 \\
\hline C & -2.96292400 & 0.80508900 & 0.22256200 \\
\hline C & -1.50552800 & -0.88154500 & 1.40204500 \\
\hline $\mathrm{H}$ & 2.17150900 & -1.79414800 & -0.53475900 \\
\hline $\mathrm{H}$ & 1.07899300 & -1.55515500 & 0.79313000 \\
\hline $\mathrm{H}$ & -0.32165500 & -2.11169700 & -1.14556700 \\
\hline $\mathrm{H}$ & 0.59591000 & -1.03760800 & -2.18375800 \\
\hline $\mathrm{H}$ & -1.32602800 & 0.22304900 & -1.87816500 \\
\hline $\mathrm{H}$ & -0.75832900 & 2.14380300 & -0.55731400 \\
\hline $\mathrm{H}$ & 1.55116200 & 2.31432500 & 0.17661600 \\
\hline $\mathrm{H}$ & 3.44612600 & 1.21605200 & 1.21262000 \\
\hline $\mathrm{H}$ & 3.95605000 & 0.23197300 & -0.16864100 \\
\hline $\mathrm{H}$ & 3.49222100 & -0.58116500 & 1.31340000 \\
\hline $\mathrm{H}$ & -2.52403100 & -1.17641900 & -0.45915800 \\
\hline $\mathrm{H}$ & -3.88360400 & 0.45395000 & 0.69030300 \\
\hline $\mathrm{H}$ & -3.23295300 & 1.26087400 & -0.73375100 \\
\hline $\mathrm{H}$ & -2.54873700 & 1.58034600 & 0.87405900 \\
\hline $\mathrm{H}$ & -2.35783900 & -1.02042500 & 2.06947900 \\
\hline $\mathrm{H}$ & -0.82911300 & -0.16929700 & 1.88760500 \\
\hline $\mathrm{H}$ & -1.00589400 & -1.84828000 & 1.31653100 \\
\hline
\end{tabular}

thermodynamic data

$\begin{array}{lc}\text { Zero-point correction= } & 0.247556 \text { (Hartree/Particle) } \\ \text { Thermal correction to Energy= } & 0.258563 \\ \text { Thermal correction to Enthalpy= } & 0.259507 \\ \text { Thermal correction to Gibbs Free Energy= } & 0.211607 \\ \text { Sum of electronic and zero-point Energies }= & -390.684162 \\ \text { Sum of electronic and thermal Energies }= & -390.673155 \\ \text { Sum of electronic and thermal Enthalpies }= & -390.672211 \\ \text { Sum of electronic and thermal Free Energies }= & -390.720110\end{array}$

E (Thermal) CV S

$\mathrm{KCal} / \mathrm{Mol} \mathrm{Cal} / \mathrm{Mol}-\mathrm{Kelvin} \mathrm{Cal} / \mathrm{Mol}-K e l v i n$ 
$\begin{array}{llll}\text { Total } & 162.250 & 42.456 & 100.814\end{array}$

\begin{tabular}{|l|l|}
\hline Terpinyl cation \\
M06-2X/6-311+G**
\end{tabular}

optimized xyz-matrix

C $\quad 1.08396300 \quad-0.97523700 \quad 0.65732000$

C $\quad-0.18495200-1.10078000 \quad-0.19213700$

$\begin{array}{llll}\text { C } & -0.70121600 & 0.40490100 & -0.63866500\end{array}$

C $\quad 0.18913900 \quad 1.51850900 \quad-0.09051100$

C $\quad \begin{array}{llll}\text { C } & 1.64235500 & 1.17958700 & -0.30938600\end{array}$

$\begin{array}{llll}\text { C } & 2.08860000 & -0.02218500 & 0.04980900\end{array}$

$\begin{array}{lllll}\text { C } & 3.50734800 & -0.48296600 & -0.07916300\end{array}$

$\begin{array}{lllll}\text { C } & -1.95059900 & 0.05063100 & -0.05440700\end{array}$

$\begin{array}{llll}\text { C } & -2.26756600 & 0.35411700 & 1.36132800\end{array}$

$\begin{array}{llll}\text { C } & -2.99650400 & -0.59007200 & -0.88409600\end{array}$

$\begin{array}{llll}\mathrm{H} & 1.50034700 & -1.98111500 & 0.75712900\end{array}$

$\begin{array}{llll}\mathrm{H} & 0.82063800 & -0.64919500 & 1.67069600\end{array}$

H $\quad-0.91711400 \quad-1.71413000 \quad 0.35087800$

$\mathrm{H} \quad-0.01914500-1.60575400 \quad-1.14339300$

$\begin{array}{llll}\mathrm{H} & -0.71877400 & 0.40045600 & -1.72636700\end{array}$

$\begin{array}{llll}\mathrm{H} & -0.00324700 & 1.68260900 & 0.97440900\end{array}$

$\begin{array}{lllll}\mathrm{H} & -0.08346500 & 2.44066000 & -0.60643000\end{array}$

H $\quad 2.29997600 \quad 1.92652800-0.73778700$

H $\quad 4.13723700 \quad 0.29035300 \quad-0.51756800$

H $\quad 3.56748400-1.37912000-0.70373700$

H $\quad 3.91450300 \quad-0.74674400 \quad 0.90126300$

H $\quad-3.04539500 \quad-0.29442000 \quad 1.76081300$

$\begin{array}{llll}\mathrm{H} & -2.64714800 & 1.38681600 & 1.36630300\end{array}$

$\begin{array}{llll}\mathrm{H} & -1.38763500 & 0.34632700 & 2.00388100\end{array}$

H $\quad-3.74296200 \quad 0.19787200 \quad-1.06685400$

H $\quad-3.51382800-1.39100700 \quad-0.35392000$

$\mathrm{H} \quad-2.62488100 \quad-0.92916400 \quad-1.84986700$

\section{thermodynamic data}

Zero-point correction=

Thermal correction to Energy=

0.246181 (Hartree/Particle)

Thermal correction to Enthalpy=

0.257579

0.258523

Thermal correction to Gibbs Free Energy=

Sum of electronic and zero-point Energies=

0.209923

Sum of electronic and thermal Energies=

$-390.664171$

Sum of electronic and thermal Enthalpies $=\quad-390.651829$

Sum of electronic and thermal Free Energies $=\quad-390.700429$ 


\begin{tabular}{|l|l|}
\hline TS-Phenandryl-Terpinyl \\
M06-2X/6-311+G**
\end{tabular}

\section{optimized xyz-matrix}

$\begin{array}{lrrr}\text { C } & -1.39266500 & 1.17815800 & 0.18325700 \\ \text { C } & -0.16361000 & 1.62148300 & -0.61917100 \\ \text { C } & 0.89061800 & 0.55200200 & -0.86110300 \\ \text { C } & 0.40515800 & -0.87100800 & -0.93075900 \\ \text { C } & -0.96252600 & -1.18776400 & -0.49923400 \\ \text { C } & -1.80142900 & -0.25504800 & -0.01733000 \\ \text { C } & -3.20239500 & -0.59891200 & 0.37881300 \\ \text { C } & 1.64258300 & -0.13572100 & 0.24491300 \\ \text { C } & 1.20729500 & 0.05114400 & 1.67916700 \\ \text { C } & 3.10475200 & -0.41124100 & -0.02150800 \\ H & -2.23937400 & 1.81984000 & -0.07435500 \\ H & -1.24108300 & 1.33239200 & 1.25760600 \\ H & 0.30165800 & 2.49254100 & -0.15278000 \\ H & -0.49351700 & 1.94489200 & -1.60875600 \\ H & 1.54797800 & 0.81084800 & -1.68638200 \\ H & 0.86963300 & -1.49015700 & -1.69644800 \\ H & -1.27826900 & -2.21989700 & -0.59542800 \\ H & -3.39940500 & -1.66777600 & 0.31256500 \\ H & -3.90683200 & -0.06935100 & -0.26930400 \\ H & -3.39959500 & -0.26176700 & 1.40046500 \\ H & 1.13369000 & -1.33888100 & 0.02719700 \\ H & 1.85359900 & -0.51367500 & 2.34994800 \\ H & 0.17516900 & -0.26404700 & 1.83731200 \\ H & 1.28239000 & 1.11424300 & 1.92462500 \\ H & 3.49945200 & -1.18642900 & 0.63475700 \\ H & 3.64360000 & 0.51972900 & 0.17996000 \\ H & 3.28422700 & -0.68105600 & -1.06325300 \\ H & & & \end{array}$

\section{thermodynamic data}

Zero-point correction=

Thermal correction to Energy=

Thermal correction to Enthalpy=

Thermal correction to Gibbs Free Energy=

Sum of electronic and zero-point Energies=

Sum of electronic and thermal Energies=

Sum of electronic and thermal Enthalpies=

Sum of electronic and thermal Free Energies=
0.245407 (Hartree/Particle)

$$
0.255840
$$

0.256785

0.210567

$-390.661446$

$-390.651012$

$-390.650068$

$-390.696286$

$\begin{array}{cccc} & \text { E (Thermal) } & \text { CV } & \text { S } \\ & \text { KCal/Mol } & \text { Cal/Mol-Kelvin } & \text { Cal/Mol-Kelvin } \\ \text { Total } & 160.542 & 41.011 & 97.274\end{array}$

$v_{\text {imag }}=-389 \mathrm{~cm}^{-1}$ 


\begin{tabular}{|l|l|}
\hline TS-Pinyl-Terpinyl & \\
M06-2X/6-311+G** & \\
&
\end{tabular}

\section{optimized xyz-matrix}

H $\quad 0.18986100 \quad-1.52368800-2.11367900$

H $\quad-1.02408300-0.29280400-2.38318700$

C $\quad 0.70359300 \quad 0.43320000 \quad-1.29960200$

H $\quad 0.81973900 \quad 1.29217600 \quad-1.95366700$

$\begin{array}{lllll}\text { C } & 1.45732000 & 0.32552300 & -0.18878900\end{array}$

$\begin{array}{llll}\text { C } & 2.42440600 & 1.38588800 & 0.24042700\end{array}$

$\begin{array}{llll}\mathrm{H} & 2.44048000 & 2.22819900 & -0.45016400\end{array}$

H $\quad 2.17109300 \quad 1.75367300 \quad 1.24040200$

$\begin{array}{llll}\mathrm{H} & 3.43421900 & 0.97074100 & 0.30774200\end{array}$

$\begin{array}{llll}\text { C } & 1.43427900 & -0.93444600 & 0.64240700\end{array}$

$\begin{array}{llll}\mathrm{H} & 2.28413400 & -1.54584200 & 0.32153000\end{array}$

$\begin{array}{llll}\text { H } & 1.65200400 & -0.68343700 & 1.68517000\end{array}$

$\begin{array}{lllll}\text { C } & 0.12964100 & -1.76867300 & 0.52097300\end{array}$

$\begin{array}{lllll}\mathrm{H} & 0.32901800 & -2.71554000 & 0.01911400\end{array}$

H $\quad-0.25505900-2.02413700 \quad 1.51029500$

C $\quad-0.91649900-1.02742800-0.31165200$

H $\quad-1.82788000-1.63882300 \quad-0.42587100$

$\begin{array}{lllll}\text { C } & & -1.36880100 & 0.25494700 & 0.31233900\end{array}$

$\begin{array}{lllll}\text { C } & -2.21576600 & 1.20649600 & -0.42294800\end{array}$

H $\quad-3.20634800 \quad 1.14499000 \quad 0.05728800$

$\mathrm{H} \quad \begin{array}{llll}\mathrm{H} & -1.87917900 & 2.23121700 & -0.24081800\end{array}$

H $\quad-2.32083700 \quad 1.01031400 \quad-1.48432800$

$\begin{array}{lllll}\text { C } & & -1.22055200 & 0.49165100 & 1.75400900\end{array}$

$\begin{array}{lllll}\mathrm{H} & -0.21940300 & 0.26045400 & 2.11993300\end{array}$

$\begin{array}{llll}H & -1.53830200 & 1.48515100 & 2.06260300\end{array}$

H $\quad-1.87915600 \quad-0.25653000 \quad 2.23089500$

\section{thermodynamic data}

Zero-point correction=

Thermal correction to Energy=

Thermal correction to Enthalpy=

Thermal correction to Gibbs Free Energy=

Sum of electronic and zero-point Energies=

Sum of electronic and thermal Energies=

Sum of electronic and thermal Enthalpies=

Sum of electronic and thermal Free Energies=
0.245849 (Hartree/Particle)

0.256295

0.257239

\subsection{5}

$-390.662467$

$-390.652022$

$-390.651078$

$-390.697692$

$\begin{array}{cccc} & \text { E (Thermal) } & \text { CV } & \text { S } \\ & \text { KCal/Mol } & \text { Cal/Mol-Kelvin } & \text { Cal/Mol-Kelvin } \\ \text { Total } & 160.828 & 41.095 & 98.108\end{array}$

$V_{\text {imag }}=-85 \mathrm{~cm}^{-1}$ 


\begin{tabular}{|l|l|l|l|}
\hline TS-Terpinyl-Bornyl & \\
M06-2X/6-311+G** & \\
\hline
\end{tabular}

\section{optimized xyz-matrix}$$
\text { C }
$$

$\mathrm{H}$

H

C

$\mathrm{H}$

C

C

$\mathrm{H}$

$\mathrm{H}$

$\mathrm{H}$

C

$\mathrm{H}$

$\mathrm{H}$

C

$\mathrm{H}$

$\mathrm{H}$

C

$\mathrm{H}$

C

C

$\mathrm{H}$

$\mathrm{H}$

$\mathrm{H}$

C

$\mathrm{H}$

$\mathrm{H}$

$\mathrm{H}$

$\begin{array}{ccc}-1.81145400 & 0.23313000 & 1.30261600 \\ -2.61607100 & 0.66294100 & 0.71917700 \\ -2.05008500 & 0.01685100 & 2.33674100 \\ -0.44771900 & 0.62921100 & 1.08135300 \\ 0.16878100 & 0.65060800 & 1.96503400 \\ 0.22992600 & 0.87708800 & -0.25011400 \\ 1.13341200 & 2.07623200 & -0.41496400 \\ 1.84340300 & 2.16091100 & 0.40997100 \\ 1.69701700 & 1.99381200 & -1.34645400 \\ 0.54453500 & 2.99435700 & -0.45611100 \\ -0.84708600 & 0.63206200 & -1.31809500 \\ -1.56009600 & 1.45822800 & -1.31634200 \\ -0.40978700 & 0.58712200 & -2.31582700 \\ -1.52006100 & -0.71494000 & -0.91118200 \\ -2.60657200 & -0.65446400 & -0.95028000 \\ -1.22720600 & -1.54542600 & -1.54348900 \\ -1.06013200 & -0.90882500 & 0.53691200 \\ -0.99362100 & -1.85349800 & 1.06145300 \\ 0.81340000 & -0.49804000 & 0.07351800 \\ 1.90230300 & -0.64430700 & 1.12882000 \\ 1.89993100 & -1.65861400 & 1.53430300 \\ 2.85849600 & -0.50970300 & 0.61703900 \\ 1.88660600 & 0.06222800 & 1.95344900 \\ 1.09580000 & -1.51902900 & -1.02743000 \\ 0.68334000 & -1.26360000 & -1.99728600 \\ 2.18083800 & -1.55584800 & -1.13798600 \\ 0.77015100 & -2.52141000 & -0.74200200\end{array}$

\section{thermodynamic data}

Zero-point correction=

Thermal correction to Energy=

Thermal correction to Enthalpy=
0.248487 (Hartree/Particle)

0.258284

0.259228
Thermal correction to Gibbs Free Energy=

Sum of electronic and zero-point Energies=

Sum of electronic and thermal Energies=

Sum of electronic and thermal Enthalpies=

Sum of electronic and thermal Free Energies=
0.214772

$-390.614451$

$-390.604655$

$-390.603711$

$-390.648166$

$\begin{array}{cccc} & \text { E (Thermal) } & \text { CV } & \text { S } \\ & \text { KCal/Mol } & \text { Cal/Mol-Kelvin } & \text { Cal/Mol-Kelvin } \\ \text { Total } & 162.076 & 40.232 & 93.565\end{array}$

$V_{\text {imag }}=-295 \mathrm{~cm}^{-1}$ 


\begin{tabular}{|l|l|}
\hline Diazo norbornane (2-N) & \\
M06-2X/6-311+G** &
\end{tabular}

01

C $-1.36071400000 .9468050000-0.0431210000$

C $0.08047600001 .4014680000-0.3607860000$

C 0.83569500000 .17524500000 .1206570000

C $-0.1476150000-0.80035700000 .7351350000$

C $-0.9515640000-1.4352050000-0.4205010000$

C $-1.7346960000-0.2136480000-0.9934850000$

H - $-1.45952700000 .0090560000-2.0264540000$

H - 2.8110080000 - $0.3941170000-0.9721130000$

H - $1.6352700000-2.1966360000-0.0356020000$

H - $0.3009070000-1.9058890000-1.1580600000$

C - 1.17597400000 .20447900001 .2939530000

H - $2.0915600000-0.28236900001 .6381640000$

H -0.77017700000 .83587500002 .0864460000$

H $0.2909110000-1.50520900001 .4398140000$

H 0.34860200002 .29912100000 .2068960000

H $0.22435200001 .6094600000-1.4245780000$

H - $2.07823900001 .7664110000-0.0406960000$

N $2.0850850000-0.0435680000-0.0507090000$

N $3.2019400000-0.2290650000-0.1928520000$

\section{Thermodynamic Data}

Zero-point correction $=0.163989$ (Hartree/Particle)

Thermal correction to Energy $=0.171228$

Thermal correction to Enthalpy $=0.172172$

Thermal correction to Gibbs Free Energy $=0.131846$

Sum of electronic and zero-point Energies $=-381.965868$

Sum of electronic and thermal Energies $=-381.958630$

Sum of electronic and thermal Enthalpies $=-381.957686$

Sum of electronic and thermal Free Energies $=-381.998011$

\begin{tabular}{rlrr} 
& E (Thermal) & CV & \multicolumn{1}{c}{ S } \\
KCal/Mol & Cal/Mol-Kelvin & Cal/Mol-Kelvin \\
Total & 107.447 & 28.713 & 84.872
\end{tabular}

\begin{tabular}{|l|l|}
\hline Norbornyl endo diazonium (endo-N-N ${ }^{+}$) & (en-2X/6-311+G* \\
\hline
\end{tabular}

11

C - $1.42929800000 .5777130000-0.5908520000$

C $-0.09008300001 .3259140000-0.7066930000$

C 0.70259900000 .71777900000 .4959180000

C $-0.2381380000-0.34570000001 .1124370000$ 
C - $0.4196380000-1.51182300000 .1248940000$

C - $1.2254530000-0.8772970000-1.0481390000$

H $-0.7029410000-0.9383800000-2.0043380000$

H - 2.1856420000 -1.3773960000 -1.1721990000

H -0.9912400000 -2.2966290000 0.6202570000

H $0.5191010000-1.9739550000-0.1905010000$

C - 1.56300600000 .42652500000 .9338720000

H - $2.4123710000-0.18679600001 .2366400000$

H - 1.59920000001 .37646000001 .4716270000

H $0.0470470000-0.62273600002 .1254050000$

H - $0.19410100002 .3977370000-0.5370230000$

H $0.41024800001 .1781890000-1.6650160000$

H - 2.2399670000 $1.1014900000-1.0927440000$

N 1.95705300000 .09499400000 .0299520000

N 2.8802020000 -0.3795110000 -0.3065370000

H 1.06639300001 .45496100001 .2153670000

\section{Thermodynamic Data}

Zero-point correction $=0.176660$ (Hartree/Particle)

Thermal correction to Energy $=0.184094$

Thermal correction to Enthalpy $=0.185038$

Thermal correction to Gibbs Free Energy $=0.144770$

Sum of electronic and zero-point Energies $=-382.325838$

Sum of electronic and thermal Energies $=-382.318404$

Sum of electronic and thermal Enthalpies $=-382.317460$

Sum of electronic and thermal Free Energies $=-\mathbf{3 8 2 . 3 5 7 7 2 8}$

$\mathrm{E}$ (Thermal)

$\mathrm{KCal} / \mathrm{Mol}$

Total 115.521
CV

Cal/Mol-Kelvin

29.985
$\mathrm{S}$

$\mathrm{Cal} / \mathrm{Mol}-$ Kelvin

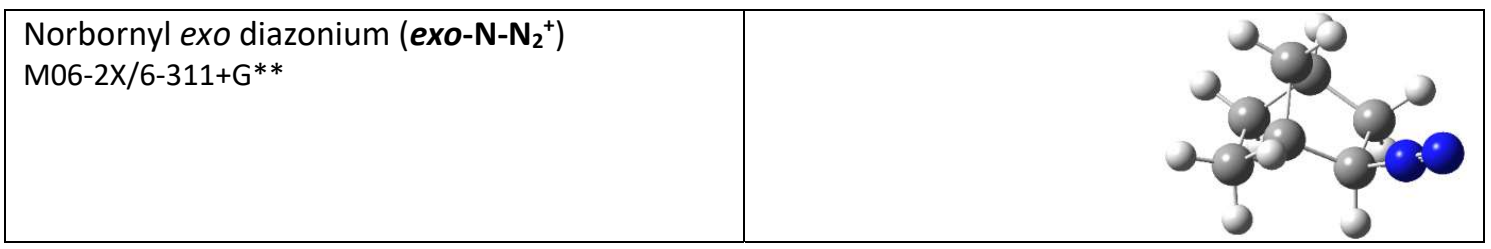

11

C - 1.07831300001 .03367900000 .4231310000

C $0.08433100001 .2860380000-0.5601470000$

C $0.7034920000-0.1394530000-0.6929550000$

C - $0.1302620000-1.01807100000 .2650650000$

C $-1.4855900000-1.1814520000-0.4637180000$

C - $2.15660500000 .2140020000-0.3046960000$

H - $2.43854900000 .6582510000-1.2598650000$

H -3.0576960000 0.14582400000 .3045820000

H - 2.0547600000 - 1.96255700000 .0400550000

H - $1.3674880000-1.4916540000-1.5036500000$

C - $0.4700170000-0.00973700001 .3740030000$

H - $1.1939290000-0.41680400002 .0804490000$

H 0.39660200000 .35366600001 .9343900000

H $0.3525640000-1.95471500000 .5396780000$

H $0.80698500001 .9959570000-0.1537340000$ 
H - $0.23190400001 .6462790000-1.5387560000$

H - 1.43646800001 .95232900000 .8826240000

N 2.1085870000 -0.1109450000 -0.1950250000

N $3.1197920000-0.10442400000 .2143990000$

H $0.8237760000-0.5290340000-1.7054810000$

\section{Thermodynamic Data}

Zero-point correction $=0.176244$ (Hartree/Particle)

Thermal correction to Energy $=0.183759$

Thermal correction to Enthalpy $=0.184703$

Thermal correction to Gibbs Free Energy $=0.144284$

Sum of electronic and zero-point Energies $=-382.326918$

Sum of electronic and thermal Energies $=-382.319403$

Sum of electronic and thermal Enthalpies $=-382.318459$

Sum of electronic and thermal Free Energies $=-\mathbf{3 8 2 . 3 5 8 8 7 9}$

$\mathrm{E}$ (Thermal)

CV

$\mathrm{S}$

$\begin{array}{llll}\text { Total } & 115.311 & 30.173 & 85.071\end{array}$

\begin{tabular}{|l|l|}
\hline $\begin{array}{l}\text { Norbornyl endo diazonium dissociation (endo- } \\
\left.\mathbf{N}-\mathbf{N}_{2}{ }^{+}-\mathrm{TS}\right) \\
\text { M06-2X/6-311+G** }\end{array}$ & \\
&
\end{tabular}

11

C $1.4569840000-0.4418560000-0.7214490000$

C $0.1881980000-1.3125850000-0.7240960000$

C $-0.4798170000-0.84862900000 .5573050000$

C 0.33704100000 .23147100001 .1656020000

C 0.35725500001 .48460100000 .2568780000

C $1.06508300001 .0107940000-1.0429540000$

H $0.42167900001 .0742380000-1.9220890000$

H $1.94929200001 .6142520000-1.2457400000$

H 0.93672500002 .25269200000 .7687220000

H -0.63938000001 .89682000000 .0931270000$

C $1.7315520000-0.39116200000 .7923270000$

H $0.4236000000-2.3736880000-0.5919960000$

$\mathrm{H}-0.4387490000-1.2297090000-1.6132020000$

H $2.2536200000-0.8475620000-1.3397880000$

N -2.1119700000 -0.0836640000 0.0349460000

N -3.0920570000 $0.2887670000-0.2615160000$

$\mathrm{H}-0.9983460000-1.56826300001 .1877270000$

H 2.5199100000 0.3013240000 1.0898150000

H $1.9255510000-1.36775700001 .2402330000$

H 0.13651200000 .41613600002 .2174990000

Thermodynamic Data

Zero-point correction $=0.173603$ (Hartree/Particle)

Thermal correction to Energy $=0.181426$

Thermal correction to Enthalpy $=0.182370$

Thermal correction to Gibbs Free Energy= 0.140467 
Sum of electronic and zero-point Energies $=-382.322411$

Sum of electronic and thermal Energies $=-382.314587$

Sum of electronic and thermal Enthalpies $=-382.313643$

Sum of electronic and thermal Free Energies $=-\mathbf{3 8 2 . 3 5 5 5 4 7}$

\begin{tabular}{rrrr} 
& E (Thermal) & CV & \multicolumn{1}{c}{ S } \\
KCal/Mol & Cal/Mol-Kelvin & Cal/Mol-Kelvin \\
Total & 113.847 & 30.002 & 88.193
\end{tabular}

$V_{\text {imag }}=-X X X \mathrm{~cm}^{-1}$

Norbornyl exo diazonium dissociation (exo-N$\mathbf{N}_{2}{ }^{+}-\mathrm{TS}$ )

11

C - 1.08621600001 .03445600000 .4387990000

C $0.00899600001 .2320350000-0.6387450000$

C $0.5812150000-0.1743560000-0.7570810000$

C - $0.1372170000-1.01853300000 .2430620000$

C $-1.5401760000-1.1521180000-0.4544090000$

C - $2.21907000000 .2201990000-0.2007330000$

H - 2.5980200000 0.6762040000 -1.1156110000

$\mathrm{H}-3.05440100000 .11899400000 .4919660000$

H -2.0628810000 -1.9736200000 0.0355510000

H - $1.4640700000-1.4114500000-1.5127850000$

C $-0.4340540000-0.00670200001 .3634930000$

H $0.76282000001 .9515580000-0.3142110000$

$\mathrm{H}-0.36406100001 .5670970000-1.6086080000$

H - 1.39240300001 .96829300000 .9039720000

N 2.2537230000 -0.0838840000 -0.1741330000

N $3.2639030000-0.07189100000 .2340020000$

H $0.8476950000-0.5880130000-1.7275950000$

$\mathrm{H}-1.1253590000-0.40864000002 .1041240000$

H 0.46141300000 .35384900001 .8771320000

H $0.3250110000-1.97372800000 .4806770000$

\section{Thermodynamic Data}

Zero-point correction $=0.174060$ (Hartree/Particle) Thermal correction to Energy $=0.181721$

Thermal correction to Enthalpy $=0.182666$

Thermal correction to Gibbs Free Energy $=0.141636$

Sum of electronic and zero-point Energies $=-382.326091$

Sum of electronic and thermal Energies $=-382.318430$

Sum of electronic and thermal Enthalpies $=-382.317486$

Sum of electronic and thermal Free Energies $=-\mathbf{3 8 2 . 3 5 8 5 1 6}$

\begin{tabular}{rrrr} 
& E (Thermal) & CV & \multicolumn{1}{c}{ S } \\
& KCal/Mol & Cal/Mol-Kelvin & Cal/Mol-Kelvin \\
Total & 114.032 & 29.742 & 86.354
\end{tabular}

$V_{\text {imag }}=-X X X \mathrm{~cm}^{-1}$ 


\begin{tabular}{|l|l|}
\hline Diazo bornane (2-B) & 0 \\
\hline
\end{tabular}

01

C - 0.96941700001 .21296600000 .5815200000

C 0.48741900001 .37163300001 .0686860000

C 1.13081300000 .27607900000 .2417140000

C $0.0637760000-0.4598350000-0.5589430000$

C $-0.35523500000 .5225680000-1.6779790000$

C - $1.01599800001 .6906770000-0.8886340000$

H $-0.47424300002 .6300810000-1.0124090000$

H - $2.04105600001 .8662250000-1.2177700000$

$\mathrm{H}-1.05991900000 .0483970000-2.3668710000$

H $0.51081600000 .8405410000-2.2606760000$

C $-1.1289160000-0.32603000000 .4461840000$

C - $2.4831620000-0.7481600000-0.1280910000$

C - $0.9122940000-1.09604800001 .7498480000$

C $0.4463950000-1.8503910000-1.0174920000$

H 0.58351100001 .19345900002 .1444190000

H 0.88299400002 .36742500000 .8519660000

H - 1.69918300001 .69634400001 .2336550000

N 2.3849300000 0.03723100000 .1578210000

N $3.5011040000-0.19100200000 .0812400000$

H $1.3048480000-1.8150340000-1.6944510000$

H - $0.3808080000-2.3185100000-1.5569510000$

H $0.7136570000-2.4847780000-0.1682560000$

H - $1.0893570000-2.16339500001 .5911930000$

H - $1.6197330000-0.75572800002 .5117950000$

H $0.0985750000-0.98496600002 .1445000000$

H -3.2651880000 - 0.58053400000 .6181460000

H - $2.4813780000-1.8166260000-0.3621860000$

H - $2.7660550000-0.2072460000-1.0304120000$

\section{Thermodynamic Data}

Zero-point correction $=0.248111$ (Hartree/Particle)

Thermal correction to Energy $=0.259505$

Thermal correction to Enthalpy $=0.260449$

Thermal correction to Gibbs Free Energy= 0.211959

Sum of electronic and zero-point Energies $=-499.801682$

Sum of electronic and thermal Energies $=-499.790287$

Sum of electronic and thermal Enthalpies $=-499.789343$

Sum of electronic and thermal Free Energies $=-\mathbf{- 4 9 9 . 8 3 7 8 3 4}$

\begin{tabular}{llrr} 
& E (Thermal) & CV & \multicolumn{1}{c}{ S } \\
& KCal/Mol & Cal/Mol-Kelvin & Cal/Mol-Kelvin \\
Total & 162.842 & 46.174 & 102.058
\end{tabular}




\begin{tabular}{|l|l|}
\hline Diazo bornane endo dissociation (endoTS-2-B) & \\
\hline
\end{tabular}

01

C $0.8125340000-1.40931700000 .2390230000$

C - $0.4258640000-1.26443200001 .1346200000$

C - 0.90981900000 .17279500000 .8874730000

C - $0.01564300000 .6620910000-0.2480150000$

C - $0.2294510000-0.1598560000-1.5459800000$

C $0.3016190000-1.5771140000-1.2038550000$

H - $0.4795420000-2.3381610000-1.2581140000$

H 1.0958040000 - $1.8831060000-1.8865190000$

H $0.34485300000 .2973830000-2.3559580000$

H - $1.2736080000-0.1537500000-1.8587570000$

C 1.34940900000 .04714800000 .2569230000

C $2.52461600000 .2933170000-0.6898360000$

C 1.77073900000 .54948100001 .6451850000

C $-0.06160200002 .1645930000-0.4554390000$

H - $0.1815380000-1.35352000002 .1971230000$

H - $1.1989860000-2.00937000000 .9372200000$

H $1.5256320000-2.17189100000 .5596020000$

N - 2.6495800000 -0.0077040000 0.1062620000

N-3.7063540000 0.28649400000 .1559090000

H - $1.05408500002 .4691920000-0.8000030000$

H $0.66589600002 .4889500000-1.2050950000$

H 0.13445200002 .69010000000 .4816390000

H 0.96823500000 .52529200002 .3832490000

H 2.1138490000 1.5852940000 1.5760480000

H 2.6053440000 -0.0496700000 2.0211610000

H $2.74912500001 .3615170000-0.7535010000$

H 2.3617500000 - $0.0785840000-1.7005720000$

H $3.4151300000-0.2034470000-0.2933060000$

\section{Thermodynamic Data}

Zero-point correction $=0.243352$ (Hartree/Particle)

Thermal correction to Energy $=0.255307$

Thermal correction to Enthalpy $=0.256251$

Thermal correction to Gibbs Free Energy $=0.206404$

Sum of electronic and zero-point Energies $=-499.753011$

Sum of electronic and thermal Energies $=-499.741056$

Sum of electronic and thermal Enthalpies $=-499.740111$

Sum of electronic and thermal Free Energies $=-499.789958$

\begin{tabular}{llrr} 
& E (Thermal) & CV & \multicolumn{1}{c}{ S } \\
KCal/Mol & Cal/Mol-Kelvin & Cal/Mol-Kelvin \\
Total & 160.207 & 47.228 & 104.911
\end{tabular}


$V_{\text {imag }}=-X X X \mathrm{~cm}^{-1}$

\begin{tabular}{|l|l|l|}
\hline Exo-bornyldiazonium dissociation TS (exo-TS-2- \\
B)
\end{tabular}

01

C - $1.1087170000-0.7661330000-1.0145470000$

C $0.2260840000-1.5396480000-0.8987360000$

C $0.8911980000-1.00747300000 .3830310000$

C -0.03378000000 .11769500000 .7792710000$

C - $1.1835240000-0.77527900001 .3767630000$

C - $1.9763100000-1.28212800000 .1439330000$

H - $2.0688030000-2.36931300000 .1273370000$

H - 2.9844220000 -0.86649300000 .1118840000$

H - $1.7912810000-0.16015200002 .0425810000$

H - $0.7701630000-1.58891400001 .9769760000$

C - $0.71038400000 .6471650000-0.5319910000$

C $-1.88955500001 .5886060000-0.2607820000$

C $0.22984100001 .3754930000-1.4964740000$

C 0.50756300001 .13648000001 .7595640000

H $0.8718190000-1.3923110000-1.7651480000$

H $0.0760400000-2.6195050000-0.8108110000$

H - $1.5791730000-0.8129730000-1.9988840000$

N 2.4392010000 -0.1674200000 -0.1506500000

N $3.5283270000-0.0647110000-0.0353790000$

H $0.71637000002 .2210520000-1.0005060000$

H - $0.34846100001 .7782270000-2.3327550000$

H $1.00825500000 .7412890000-1.9178430000$

H - 2.55295500001 .24547000000 .5332080000

$\mathrm{H}-2.48891400001 .7154910000-1.1671410000$

H - 1.51827200002 .57670600000 .0262280000

H - 0.28497200001 .80842700002 .1002380000

H 1.29466100001 .74568000001 .3056950000

H 0.93307000000 .63357000002 .6309610000

\section{Thermodynamic Data}

Zero-point correction $=0.243523$ (Hartree/Particle)

Thermal correction to Energy $=0.255278$

Thermal correction to Enthalpy $=0.256222$

Thermal correction to Gibbs Free Energy $=0.207248$

Sum of electronic and zero-point Energies $=-499.758761$

Sum of electronic and thermal Energies $=-499.747006$

Sum of electronic and thermal Enthalpies $=-499.746062$

Sum of electronic and thermal Free Energies $=-499.795036$
$\mathrm{E}$ (Thermal)
CV
$\mathrm{S}$
Total 160.189
Cal/Mol-Kelvin
47.086
Cal/Mol-Kelvin
103.076 
$V_{\text {imag }}=-X X X \mathrm{~cm}^{-1}$

\begin{tabular}{|l|l|}
\hline endo bornane diazonium $\left(\right.$ endo-B- $\left.\mathbf{N}_{2}{ }^{+}\right)$ & (1) \\
\hline
\end{tabular}

11

C - 0.75217100001 .40615900000 .2685740000

C 0.50951300001 .28769200001 .1454090000

C $0.9754500000-0.15983100000 .8001210000$

C $0.0215980000-0.6601560000-0.3249360000$

C $0.27274100000 .2176170000-1.5706780000$

C - $0.28276900001 .6169530000-1.1810360000$

H $0.46793500002 .4050350000-1.2553790000$

H -1.1113450000 1.9060030000 -1.8258440000

H - $0.2614550000-0.2116840000-2.4180970000$

H $1.32386500000 .2395690000-1.8726470000$

C - $1.3030380000-0.04369300000 .2543080000$

C - $2.4931100000-0.2491490000-0.6865250000$

C - $1.7120030000-0.57701300001 .6321420000$

C $0.0786920000-2.1565070000-0.5491710000$

H 0.30785800001 .34935100002 .2132910000

H 1.26124300002 .03923100000 .8998610000

H - 1.44512700002 .16145600000 .6386650000

N $2.3663010000-0.16870800000 .3290490000$

N $3.3851910000-0.1815790000-0.0627660000$

H $1.0479300000-2.4707840000-0.9487930000$

H $-0.6767620000-2.4473490000-1.2815750000$

H $-0.1092230000-2.71157900000 .3732340000$

H -1.9705500000 -1.6366310000 1.5717640000

H - 2.6040990000 -0.0466500000 1.9721030000

H $-0.9607500000-0.46142400002 .4163800000$

H -3.3370150000 $0.3489460000-0.3351910000$

H - $2.8086770000-1.2949120000-0.6750520000$

H - $2.29523600000 .0317150000-1.7195370000$

H $1.0215450000-0.84071600001 .6535760000$

\section{Thermodynamic Data}

Zero-point correction $=0.260071$ (Hartree/Particle)

Thermal correction to Energy $=0.271795$

Thermal correction to Enthalpy $=0.272739$

Thermal correction to Gibbs Free Energy= 0.223948

Sum of electronic and zero-point Energies $=-500.165645$

Sum of electronic and thermal Energies $=-500.153921$

Sum of electronic and thermal Enthalpies $=-500.152977$

Sum of electronic and thermal Free Energies $=-500.201767$

$$
\begin{array}{lcc}
\mathrm{E}(\text { Thermal) } & \mathrm{CV} & \mathrm{S} \\
\mathrm{KCal} / \mathrm{Mol} & \mathrm{Cal} / \text { Mol-Kelvin } & \mathrm{Cal} / \text { Mol-Kelvin }
\end{array}
$$




\begin{tabular}{|l|l|}
\hline exo bornane diazonium $\left(\right.$ exo-B- $\left.\mathbf{N}_{2}{ }^{+}\right)$ & \\
\hline
\end{tabular}

\section{1}

C - 1.05949400000 .75886200001 .0274750000

C 0.27725100001 .53121100000 .9758750000

C $0.92573400000 .9446820000-0.3104090000$

C - $0.0588930000-0.1520880000-0.8034620000$

C - $1.20697700000 .7263500000-1.3792670000$

C - $1.92804400001 .2920810000-0.1244440000$

H - $1.98091300002 .3811330000-0.1256740000$

H - $2.94908700000 .9202070000-0.0521820000$

H - $1.86124400000 .0959970000-1.9814500000$

H - $0.82909800001 .5035990000-2.0479730000$

C - $0.6675190000-0.65970300000 .5418560000$

C - $1.8580550000-1.59733100000 .3165720000$

C $0.3090920000-1.39000000001 .4720310000$

C $0.5116630000-1.1623290000-1.7770560000$

H 0.89704400001 .35902200001 .8551240000

H 0.14853000002 .60763600000 .8658140000

H - 1.52535300000 .80445200002 .0117000000

N 2.2526840000 $0.3431670000-0.0123680000$

N $3.2257080000-0.12123400000 .1565180000$

H $0.9106410000-0.6771470000-2.6720980000$

H - $0.2827720000-1.8379370000-2.0985040000$

H $1.2995590000-1.7772860000-1.3323610000$

H $0.7612260000-2.25081900000 .9713630000$

H - $0.2387570000-1.77776000002 .3331460000$

H $1.1079690000-0.77075000001 .8843790000$

H - 2.3919270000 - 1.74289500001 .2581500000

H - $1.5069790000-2.5779030000-0.0141550000$

H - $2.5743660000-1.2339130000-0.4185970000$

H $1.19824100001 .6704240000-1.0807650000$

\section{Thermodynamic Data}

Zero-point correction $=0.260281$ (Hartree/Particle)

Thermal correction to Energy $=0.271880$

Thermal correction to Enthalpy $=0.272824$

Thermal correction to Gibbs Free Energy $=0.224484$

Sum of electronic and zero-point Energies $=-500.165191$

Sum of electronic and thermal Energies $=-500.153593$

Sum of electronic and thermal Enthalpies $=-500.152648$

Sum of electronic and thermal Free Energies $=-500.200988$

\begin{tabular}{llrr} 
& E (Thermal) & CV & \multicolumn{1}{c}{ S } \\
KCal/Mol & Cal/Mol-Kelvin & Cal/Mol-Kelvin \\
Total & 170.607 & 47.664 & 101.740
\end{tabular}




\begin{tabular}{|l|l|}
\hline $\begin{array}{l}\text { endo bornane diazonium dissociation (endo-B- } \\
\mathrm{N}^{+}{ }^{+} \text {-TS) }\end{array}$ & \\
&
\end{tabular}

11

C $0.8055770000-1.41479300000 .2510030000$

C - $0.4648530000-1.29120300001 .1304090000$

C -0.87809100000 .14817500000 .8105780000$

C $-0.02335600000 .6711230000-0.3140900000$

C - $0.2512820000-0.2177850000-1.5713200000$

C $0.3363190000-1.6128980000-1.2032330000$

H - $0.3983730000-2.4156440000-1.2879400000$

H $1.1669930000-1.8785470000-1.8562150000$

H $0.26347400000 .2324890000-2.4195880000$

H - $1.3072580000-0.2593690000-1.8517250000$

C 1.35581500000 .04680100000 .2616460000

C $2.53227700000 .2967590000-0.6932050000$

C 1.77951500000 .56457400001 .6499640000

C - $0.09713500002 .1716430000-0.5445720000$

H - $0.2729730000-1.38093300002 .1999170000$

H - $1.2248090000-2.03155900000 .8768340000$

H $1.4938500000-2.17771400000 .6139260000$

$\mathrm{N}-2.55726600000 .13127900000 .2928140000$

N $-3.61053000000 .1614870000-0.0071220000$

H - $1.07040800002 .4665100000-0.9496220000$

H $0.65469900002 .4814020000-1.2721390000$

H 0.07028400002 .73731600000 .3755390000

H 2.01058300001 .63156300001 .6096720000

H 2.69117900000 .05084000001 .9636340000

H 1.04660400000 .41749400002 .4464720000

H $3.3910470000-0.2891460000-0.3539630000$

H $2.83044000001 .3472510000-0.6728590000$

H $2.33568800000 .0231880000-1.7280440000$

H -1.0751730000 0.82111600001 .6431760000

\section{Thermodynamic Data}

Zero-point correction $=0.257120$ (Hartree/Particle)

Thermal correction to Energy $=0.269025$

Thermal correction to Enthalpy $=0.269970$

Thermal correction to Gibbs Free Energy $=0.220749$

Sum of electronic and zero-point Energies $=-500.162342$

Sum of electronic and thermal Energies $=-500.150436$

Sum of electronic and thermal Enthalpies $=-500.149492$

Sum of electronic and thermal Free Energies $=-500.198712$

\begin{tabular}{llrr} 
& E (Thermal) & CV & \multicolumn{1}{c}{ S } \\
KCal/Mol & Cal/Mol-Kelvin & Cal/Mol-Kelvin \\
Total & 168.816 & 47.748 & 103.593
\end{tabular}




$$
V_{\text {imag }}=-X X X \mathrm{~cm}^{-1}
$$

\begin{tabular}{|l|l|}
\hline exo bornane diazonium dissociation $\left(\right.$ exo- $-\mathbf{B}^{-} \mathbf{N}_{2}{ }^{+}$ & \\
TS) &
\end{tabular}

\section{1}

C - $1.0822610000-0.7178870000-1.0640020000$

C $0.2774450000-1.4707640000-1.0348450000$

C $0.8822620000-0.94855800000 .2754310000$

C -0.06409700000 .11746700000 .8090330000$

C - $1.2116340000-0.83519200001 .3388820000$

C - $1.9434120000-1.32872300000 .0587610000$

H - $1.9879700000-2.4172260000-0.0049950000$

H - $2.9697440000-0.96591300000 .0189760000$

H - $1.8552500000-0.24094200001 .9877840000$

H - $0.8241160000-1.64879600001 .9578730000$

C $-0.71259400000 .6925730000-0.5093790000$

C - $1.92580500001 .5978910000-0.2342040000$

C $0.24355000001 .4908040000-1.4138410000$

C 0.48765100001 .06640200001 .8602990000

H $0.8947160000-1.2311780000-1.9001260000$

H $0.1799000000-2.5570830000-1.0005930000$

H - $1.5392270000-0.7301200000-2.0531850000$

N 2.3887110000 -0.2794600000 -0.0422090000

N $3.40521300000 .1134190000-0.1589640000$

H 0.90345300000 .52563900002 .7154830000

H -0.31067500001 .70633200002 .2389500000$

H 1.26562000001 .72225200001 .4584230000

H $0.62345800002 .3772480000-0.8993840000$

H - $0.29787500001 .8432390000-2.2947800000$

H $1.09860000000 .9277870000-1.7932910000$

H - $2.45584300001 .7962100000-1.1688520000$

H - 1.60071700002 .56289900000 .1624420000

H - 2.64588500001 .17929500000 .4674000000

H $1.2574560000-1.67142500000 .9992660000$

\section{Thermodynamic Data}

Zero-point correction $=0.257881$ (Hartree/Particle) Thermal correction to Energy $=0.269431$

Thermal correction to Enthalpy $=0.270375$

Thermal correction to Gibbs Free Energy $=0.222130$

Sum of electronic and zero-point Energies $=-500.164373$

Sum of electronic and thermal Energies $=-500.152823$

Sum of electronic and thermal Enthalpies $=-500.151878$

Sum of electronic and thermal Free Energies $=-500.200124$

$$
\begin{array}{lcc}
\text { E (Thermal) } & \text { CV } & \text { S } \\
\text { KCal/Mol } & \text { Cal/Mol-Kelvin } & \text { Cal/Mol-Kelvin }
\end{array}
$$




\begin{tabular}{|l|l|}
\hline fenchane endo-diazonium $\left(\right.$ endo- $\left.\mathbf{F}-\mathrm{N}_{2}{ }^{+}\right)$ & \\
\hline
\end{tabular}

11

C $-0.5818570000-1.5172250000-0.1933790000$

C $-1.2558700000-0.17401700000 .2186810000$

C 0.03631200000 .62593400000 .6634080000

C $1.2609780000-0.25039700000 .2809990000$

C $1.2857690000-0.4203170000-1.2563980000$

C $0.1118560000-1.3920970000-1.5578280000$

H - $0.5426240000-1.0515210000-2.3578880000$

H $0.4947180000-2.3689030000-1.8552090000$

H $2.2470160000-0.8478240000-1.5449430000$

H $1.20567600000 .5315710000-1.7919050000$

C $0.6421030000-1.59076600000 .7362650000$

H $1.3071150000-2.42238500000 .4968250000$

H $0.4143720000-1.62223700001 .8038850000$

C 2.55931400000 .19663700000 .9209060000

C - $2.1598590000-0.35498600001 .4404720000$

C - $2.04458700000 .5058610000-0.9002260000$

H - $1.2878490000-2.3448020000-0.1233590000$

N 0.12183800001 .93374000000 .0178030000

N $0.20628100002 .8908830000-0.5012000000$

H 2.46391900000 .28437700002 .0060440000

H 2.90065700001 .15732900000 .5226560000

H $3.3414740000-0.53501400000 .7107230000$

H - $2.56809100001 .3922190000-0.5311220000$

H - $2.8024750000-0.1872210000-1.2705010000$

H - $1.42645000000 .7940900000-1.7534830000$

H -3.0358070000 -0.9416050000 1.1568400000

$\mathrm{H}-2.51247600000 .60865500001 .8177220000$

H - $1.6549460000-0.87977300002 .2537690000$

H 0.03398100000 .88891300001 .7263280000

\section{Thermodynamic Data}

Zero-point correction $=0.260086$ (Hartree/Particle)

Thermal correction to Energy $=0.271672$

Thermal correction to Enthalpy $=0.272616$

Thermal correction to Gibbs Free Energy= 0.224215

Sum of electronic and zero-point Energies $=-500.168473$

Sum of electronic and thermal Energies $=-500.156888$

Sum of electronic and thermal Enthalpies $=-500.155943$

Sum of electronic and thermal Free Energies $=-500.204344$

$\begin{array}{llrr} & \text { E (Thermal) } & \text { CV } & \text { S } \\ & \text { KCal/Mol } & \text { Cal/Mol-Kelvin } & \text { Cal/Mol-Kelvin } \\ \text { Total } & 170.476 & 47.609 & 101.868\end{array}$




fenchane exo-diazonium $\left(\right.$ exo-F- $\left.\mathbf{N}_{2}{ }^{+}\right)$

11

C - $1.2585120000-0.23735700000 .9113520000$

C - $0.83138100000 .9265800000-0.0354100000$

C $0.46596200000 .2903210000-0.6796400000$

C $0.6221730000-1.1134580000-0.0300650000$

C $-0.5505410000-1.9186470000-0.6573030000$

C - $1.8081660000-1.41946700000 .1008220000$

H - $2.6343380000-1.1540950000-0.5559600000$

H - $2.1747560000-2.18682700000 .7834060000$

H - $0.3669790000-2.9800760000-0.4851230000$

H $-0.6108410000-1.7791500000-1.7402540000$

C $0.0867920000-0.81319000001 .3809250000$

H - $0.0261200000-1.72688000001 .9669790000$

H $0.7126190000-0.11955800001 .9499900000$

C $1.9847120000-1.7610800000-0.1778060000$

C -0.55438400002 .19532500000 .7782370000$

C - $1.82710400001 .2552460000-1.1493050000$

H -1.9274900000 0.12159700001 .6934330000

N $1.65663700001 .0947860000-0.3329640000$

N $2.53770300001 .6647750000-0.0319970000$

H $2.7756550000-1.19599600000 .3237040000$

H 2.2558540000 -1.8911200000 -1.2289590000

H $1.9608390000-2.75040900000 .2818140000$

H - $1.51305800002 .1464690000-1.6979060000$

H - 2.8072930000 $1.4591020000-0.7123490000$

H - $1.94187100000 .4396030000-1.8653170000$

$\mathrm{H}-1.50420600002 .56885900001 .1635620000$

$\mathrm{H}-0.12548800002 .99038500000 .1614880000$

H 0.09598600002 .01899300001 .6382960000

H $0.49380200000 .2965460000-1.7729150000$

\section{Thermodynamic Data}

Zero-point correction $=0.259815$ (Hartree/Particle)

Thermal correction to Energy $=0.271405$

Thermal correction to Enthalpy $=0.272349$

Thermal correction to Gibbs Free Energy $=0.223989$

Sum of electronic and zero-point Energies $=-500.169052$

Sum of electronic and thermal Energies $=-500.157462$

Sum of electronic and thermal Enthalpies $=-500.156517$

Sum of electronic and thermal Free Energies $=-\mathbf{5 0 0 . 2 0 4 8 7 7}$

\begin{tabular}{llrr} 
& E (Thermal) & CV & \multicolumn{1}{c}{ S } \\
KCal/Mol & Cal/Mol-Kelvin & Cal/Mol-Kelvin \\
Total & 170.309 & 47.704 & 101.782
\end{tabular}




\begin{tabular}{|l|l|}
\hline fenchane endo-diazonium dissociation (endo-F- \\
$\mathbf{N}^{+}$-TS)
\end{tabular}

11

C $0.7451050000-1.47712800000 .3028780000$

C $1.2771370000-0.0925110000-0.2125160000$

C $-0.07471500000 .4892340000-0.7007940000$

C $-1.2164600000-0.4063920000-0.2963980000$

C - $1.2734160000-0.53060900001 .2553710000$

C $0.0043480000-1.33103900001 .6449860000$

H $0.5940140000-0.84966500002 .4238060000$

H - $0.2621150000-2.32036700002 .0209050000$

H - 2.1847960000 -1.0654350000 1.5260130000

H - 1.33345400000 .44685900001 .7420290000

C $-0.4369560000-1.7306710000-0.6564030000$

$\mathrm{H}-1.0532120000-2.5910660000-0.3877160000$

H - $0.1709390000-1.8210320000-1.7108980000$

C - $2.5420970000-0.1461300000-0.9944730000$

C $2.1977150000-0.2663850000-1.4411050000$

C 2.02028500000 .74883700000 .8379500000

H $1.5304440000-2.23234200000 .3062310000$

N -0.35126900002 .08910800000 .0256350000$

$\mathrm{N}-0.58765900003 .10079700000 .3725510000$

H - $2.4269150000-0.0918460000-2.0803490000$

H -3.0015020000 $0.7862600000-0.6520100000$

H -3.2463000000 -0.9505150000 -0.7734860000

H 2.40294800001 .67495000000 .4011510000

H 2.88108600000 .18397600001 .2010290000

H 1.40517300000 .99984400001 .7033740000

H $3.1227670000-0.7555860000-1.1292630000$

H $2.46264700000 .7023040000-1.8737390000$

H $1.7502210000-0.8786190000-2.2260020000$

H - $0.10324900000 .8897140000-1.7153470000$

\section{Thermodynamic Data}

Zero-point correction $=0.257047$ (Hartree/Particle)

Thermal correction to Energy $=0.268817$

Thermal correction to Enthalpy $=0.269761$

Thermal correction to Gibbs Free Energy $=0.220862$

Sum of electronic and zero-point Energies $=-500.164495$

Sum of electronic and thermal Energies $=-500.152725$

Sum of electronic and thermal Enthalpies $=-500.151781$

Sum of electronic and thermal Free Energies $=-500.200680$

\begin{tabular}{|c|c|c|c|}
\hline & $\mathrm{E}$ (Thermal) & $\begin{array}{c}C V \\
\text { C }\end{array}$ & $\begin{array}{c}S \\
\text { Cand }\end{array}$ \\
\hline & $\mathrm{KCal} / \mathrm{Mol}$ & Cal/Mol-Kelvin & Cal/Mol-Kelvin \\
\hline Total & 168.685 & 47.567 & 102.916 \\
\hline
\end{tabular}




\begin{tabular}{|l|l|}
\hline $\begin{array}{l}\text { fenchane exo-diazonium dissociation (exo-F- } \\
\mathbf{N}^{+} \text {-TS) }\end{array}$ & \\
\hline
\end{tabular}

\section{1}

C $1.2898070000-0.37185800000 .8968380000$

C $0.3259740000-1.2035190000-0.0346500000$

C - $0.4819010000-0.0322650000-0.6622050000$

C $-0.01876700001 .2669300000-0.0187010000$

C $1.40252100001 .4367410000-0.6907740000$

C 2.3069690000 0.41477300000 .0489730000

H $2.8939140000-0.2076940000-0.6249000000$

H 3.01314800000 .92608100000 .7052530000

H $1.71530100002 .4684090000-0.5211940000$

H $1.37509000001 .2941040000-1.7745700000$

C 0.37784900000 .76833200001 .3918020000

H 0.91857200001 .53081000001 .9545910000

H - 0.47354100000 .44585600001 .9959170000

C - $0.91772300002 .4851750000-0.1638290000$

C - $0.5150270000-2.17537000000 .8186350000$

C $1.0165280000-2.0086660000-1.1505710000$

H $1.7342940000-1.00227900001 .6664700000$

N - 2.1287600000 -0.2163500000 -0.2927800000

N -3.1997910000 -0.2599570000 -0.0658540000

$\mathrm{H}-1.87075300002 .36237300000 .3588200000$

H - $1.12485200002 .7152300000-1.2128960000$

H -0.42562600003 .35649700000 .2719660000$

H $0.2930560000-2.6312690000-1.6833910000$

H $1.7655630000-2.6724520000-0.7132500000$

H $1.5206210000-1.3789900000-1.8851380000$

H $0.1468770000-2.94196600001 .2245090000$

$\mathrm{H}-1.2687410000-2.68867900000 .2150850000$

$\mathrm{H}-1.0076670000-1.69033200001 .6634260000$

H - $0.6227780000-0.0331890000-1.7433810000$

\section{Thermodynamic Data}

Zero-point correction $=0.257722$ (Hartree/Particle)

Thermal correction to Energy $=0.269217$

Thermal correction to Enthalpy $=0.270161$

Thermal correction to Gibbs Free Energy= 0.221992

Sum of electronic and zero-point Energies $=-500.167404$

Sum of electronic and thermal Energies $=-500.155910$

Sum of electronic and thermal Enthalpies $=-500.154965$

Sum of electronic and thermal Free Energies $=-500.203134$

\begin{tabular}{llrr} 
& E (Thermal) & CV & \multicolumn{1}{c}{ S } \\
KCal/Mol & Cal/Mol-Kelvin & Cal/Mol-Kelvin \\
Total & 168.936 & 47.106 & 101.379
\end{tabular}


$\boldsymbol{V}_{\text {imag }}=-X X X \mathrm{~cm}^{-1}$

4.2.2. Structures optimized at the M06-2X/6-31G* level of theory

\begin{tabular}{|l|l|l|}
\hline Norbornyl cation $\left(\mathbf{N}^{+}\right)$ & &
\end{tabular}

optimized xyz-matrix

C $0.9802780000-0.67097800000 .0000040000$

C $0.0267990000-0.8495390000-1.1958650000$

C - $1.1246610000-0.0077670000-0.6968410000$

C - $-1.1245310000-0.00755500000 .6969780000$

C $-0.17287600001 .3918360000-0.0001160000$

C $1.25228400000 .8421410000-0.0001490000$

H $1.79974700001 .1642030000-0.8880100000$

H 1.79985200001 .16435800000 .8875920000

H -0.48069500001 .98322600000 .8680720000$

H - $0.48073800001 .9829840000-0.8684600000$

C $0.0269580000-0.84930800001 .1960150000$

H $0.4303010000-0.51456700002 .1537810000$

H - $0.3263790000-1.88296400001 .3008360000$

H - 1.94591200000 .35950200001 .3046270000

H - $1.94611900000 .3592030000-1.3044420000$

H - $0.3265270000-1.8832330000-1.3003860000$

H $0.4299920000-0.5149940000-2.1537600000$

H $1.8609720000-1.3107000000-0.0000020000$

\section{Thermodynamic Data}

Zero-point correction $=0.166365$ (Hartree/Particle)

Thermal correction to Energy $=0.172020$

Thermal correction to Enthalpy $=0.172964$

Thermal correction to Gibbs Free Energy $=0.137533$

Sum of electronic and zero-point Energies $=-272.764734$

Sum of electronic and thermal Energies $=-272.759080$

Sum of electronic and thermal Enthalpies $=-272.758135$

Sum of electronic and thermal Free Energies $=-272.793566$

\begin{tabular}{lllc} 
& E (Thermal) & \multicolumn{1}{c}{ CV } & S \\
& KCal/Mol & Cal/Mol-Kelvin & Cal/Mol-Kelvin \\
Total & 107.944 & 23.720 & 74.57
\end{tabular}

\begin{tabular}{|l|l|}
\hline Bornyl cation $\left(\mathbf{B}^{+}\right)$ & \\
\hline
\end{tabular}

optimized xyz-matrix

C $1.3938390000-0.5211030000-1.2607020000$ 
H 2.3535650000 -0.0298920000 -1.4878380000

H $1.3383930000-1.3829120000-1.9350100000$

C $0.25754000000 .3955340000-1.5194560000$

H - $0.14764700000 .5541540000-2.5221550000$

C $-0.37174100000 .8686360000-0.3491110000$

C - $1.68725500001 .6031280000-0.4549360000$

H - 2.3689720000 $1.1225950000-1.1622250000$

H - 2.1752580000 1.64622300000 .5225560000

H - $1.52284400002 .6341150000-0.7829700000$

C 0.68750900001 .47439000000 .6403890000

H 1.19254200002 .30856000000 .1485500000

H 0.12914900001 .88997200001 .4824880000

C 1.64999400000 .33916900001 .0522820000

H 2.69206000000 .58007800000 .8321000000

H 1.58608800000 .12947700002 .1219440000

C $1.1594670000-0.85174500000 .2229040000$

H $1.5632270000-1.81800200000 .5325100000$

C $-0.3885830000-0.75142900000 .2870340000$

C - $1.1665170000-1.7666100000-0.5502250000$

H - $0.7544640000-1.9547130000-1.5444820000$

H - $1.1808460000-2.7206060000-0.0140580000$

H - $2.2065900000-1.4478060000-0.6680170000$

C - $1.0230520000-0.68669200001 .6674430000$

H -0.63618400000 .10085600002 .3135100000$

H - $2.1086150000-0.58263900001 .5938180000$

H - $0.8208090000-1.64912000002 .1555470000$

\section{Thermodynamic data}

Zero-point correction $=0.249360$ (Hartree/Particle)

Thermal correction to Energy $=0.259150$

Thermal correction to Enthalpy $=0.260094$

Thermal correction to Gibbs Free Energy $=0.215850$

Sum of electronic and zero-point Energies $=-390.552743$

Sum of electronic and thermal Energies $=-390.542953$

Sum of electronic and thermal Enthalpies $=-390.542009$

Sum of electronic and thermal Free Energies $=-\mathbf{3 9 0 . 5 8 6 2 5 3}$
E (Thermal)
CV
$S$
$\mathrm{KCal} / \mathrm{Mol}$
Cal/Mol-Kelvin
$\mathrm{Cal} / \mathrm{Mol}-\mathrm{Kelvin}$

Total 162.619

40.180

93.119

\begin{tabular}{|l|l|}
\hline Fenchyl cation $\left(\mathbf{F}^{+}\right)$ & \\
& \\
\hline
\end{tabular}

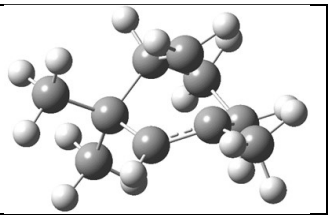

optimized xyz-matrix

C $0.6244800000-0.30523400001 .3351750000$

H 1.46252500000 .08745000001 .9220900000 
H $0.3200390000-1.26684900001 .7569690000$ C $0.0027450000-0.9259910000-0.5931180000$

H - $0.0688550000-1.9128970000-1.0638460000$

C $1.2478840000-0.3568990000-0.2067210000$

C $2.5187680000-1.1426960000-0.4406160000$

H $2.3808350000-2.2104820000-0.2515910000$

H $2.8602930000-1.0126130000-1.4716410000$

H $3.3100660000-0.77640400000 .2187360000$

C $1.33386100001 .1838470000-0.4558290000$

H $2.33267200001 .4938890000-0.1373500000$

H $1.25756200001 .3805640000-1.5280680000$

C 0.22009700001 .85115300000 .3804410000

H 0.64841100002 .46566500001 .1756000000

H - $0.42871400002 .4969580000-0.2134330000$

C -0.52331800000 .65893900000 .9871580000$

H - 1.19356000000 .91258200001 .8105780000

C - $1.2058760000-0.2050200000-0.1119130000$

C - $2.2143020000-1.20222300000 .4939670000$

H -3.0914970000 -0.6418100000 0.8291930000

H - 2.5405000000 -1.9326520000 -0.2520900000

H - $1.8094620000-1.74060800001 .3549100000$

C - $1.87874600000 .5303570000-1.2928930000$

H -1.1892790000 $1.1768540000-1.8401380000$

H - 2.3167950000 - $0.1850970000-1.9938020000$

H - $2.68730300001 .1480590000-0.8900300000$

\section{Thermodynamic data}

Zero-point correction $=0.249697$ (Hartree/Particle)

Thermal correction to Energy $=0.259497$

Thermal correction to Enthalpy $=0.260441$

Thermal correction to Gibbs Free Energy $=0.216043$

Sum of electronic and zero-point Energies $=-390.551925$

Sum of electronic and thermal Energies $=-390.542126$

Sum of electronic and thermal Enthalpies $=-390.541182$

Sum of electronic and thermal Free Energies $=-390.585580$
$\mathrm{E}$ (Thermal)
$\mathrm{CV}$
$\mathrm{S}$
$\mathrm{KCal} / \mathrm{Mol} \quad \mathrm{Cal} / \mathrm{Mol}-\mathrm{Kelvin} \quad \mathrm{Cal} / \mathrm{Mol}-K e l v i n$

$\begin{array}{lll}\text { Total } & 162.837 & 40.003\end{array}$

\begin{tabular}{|l|l|}
\hline Camphyl cation $\left(\mathbf{C}^{+}\right)$ & \\
\hline
\end{tabular}

\section{optimized xyz matrix}

C $0.92225200000 .9390860000-0.8040540000$

C - $0.37751700000 .8776430000-0.2196500000$

C $-0.7279330000-0.54939200000 .0591320000$

C $0.6665260000-1.2191430000-0.1229310000$

C $1.5655470000-0.68787600001 .0077330000$

C 1.53500300000 .82497000000 .7433340000 
H 0.99526300001 .40180600001 .5017990000 H 2.5086730000 1.30661600000 .6195930000 H $2.5791100000-1.07958100000 .8998180000$ H $1.2131600000-0.94193200002 .0079910000$ C $1.2506890000-0.4623880000-1.3262000000$ H $2.3259030000-0.6143980000-1.4432110000$ H $0.7658900000-0.6652940000-2.2828680000$ H $0.6155830000-2.3055200000-0.2094630000$ C - $1.7067260000-0.9741640000-1.0749510000$ C - $1.4067480000-0.78338700001 .4117500000$ C - 1.24183600002 .03119300000 .0879530000 H $1.24718600001 .8481720000-1.3052330000$ H - $2.05376600002 .0056190000-0.6567360000$ H - $0.72559200002 .9868730000-0.0145640000$ $\mathrm{H}-1.71615500001 .94301900001 .0693680000$ H - 2.6722120000 -0.4771550000 -0.9516670000 H - $1.8646000000-2.0525530000-0.9848500000$ H - $1.3348390000-0.7660560000-2.0800640000$ H -1.4705930000 -1.8586760000 1.6023780000 H - $2.4256140000-0.38645700001 .4133140000$ H - $-0.8629330000-0.32374500002 .2416990000$

\section{Thermodynamic data}

Zero-point correction $=0.251006$ (Hartree/Particle) Thermal correction to Energy $=0.261158$ Thermal correction to Enthalpy $=0.262102$ Thermal correction to Gibbs Free Energy $=0.216823$ Sum of electronic and zero-point Energies $=-390.582068$ Sum of electronic and thermal Energies $=-390.571917$ Sum of electronic and thermal Enthalpies $=-390.570973$ Sum of electronic and thermal Free Energies $=-\mathbf{3 9 0 . 6 1 6 2 5 2}$

\begin{tabular}{rlrr} 
& E (Thermal) & CV & \multicolumn{1}{c}{ S } \\
KCal/Mol & Cal/Mol-Kelvin & Cal/Mol-Kelvin \\
Total & 163.879 & 40.995 & 95.298
\end{tabular}

\begin{tabular}{|l|l|}
\hline Pinyl cation $\left(\mathbf{P}^{+}\right)$ & 1 \\
\hline & \\
\hline
\end{tabular}

11

C 0.86181500001 .18248800000 .2563330000

C $0.62501800001 .2220240000-1.2685320000$

C $-0.0342610000-0.1518480000-1.2553770000$

C $-1.1774330000-0.3004560000-0.4713200000$

C - 1.62206600000 .87082000000 .3446920000

C -0.41057800001 .64512400000 .9526240000$

H -0.54491500002 .72012600000 .8151800000$

$\mathrm{H}-0.35674400001 .45849900002 .0282160000$

H - 2.34870000000 .56780200001 .1025250000

H - 2.1545570000 $1.5276340000-0.3592500000$ 
C $0.9381810000-0.35539600000 .2884950000$

C $0.4809410000-1.11571300001 .5196900000$

C 2.2152670000 -0.9775320000 -0.2554150000

C - $1.9449580000-1.5691720000-0.4353440000$

H $0.1991400000-0.9256280000-1.9847660000$

H $1.54238000001 .2197360000-1.8547490000$

H -0.0177520000 2.0327730000 -1.6228990000

H 1.75466600001 .68503000000 .6393380000

H $2.9684080000-0.90820400000 .5389860000$

H $2.6104350000-0.4744920000-1.1381300000$

H $2.0713470000-2.0372870000-0.4813340000$

H -0.4517160000 -0.7625490000 1.9621050000

H $1.2676870000-0.98786200002 .2733740000$

H $0.3971920000-2.18758900001 .3179430000$

H - $1.4499790000-2.3789450000-0.9745180000$

H - $2.9174300000-1.3804840000-0.9120680000$

H - 2.1610110000 -1.8705870000 0.5949690000

\section{Thermodynamic data}

Zero-point correction $=0.250874$ (Hartree/Particle)

Thermal correction to Energy $=0.261059$

Thermal correction to Enthalpy $=0.262004$

Thermal correction to Gibbs Free Energy $=0.216890$

Sum of electronic and zero-point Energies $=-390.565963$

Sum of electronic and thermal Energies $=-390.555778$

Sum of electronic and thermal Enthalpies $=-390.554834$

Sum of electronic and thermal Free Energies $=-\mathbf{3 9 0 . 5 9 9 9 4 8}$

\begin{tabular}{rrrr} 
& E (Thermal) & CV & \multicolumn{1}{c}{ S } \\
KCal/Mol & Cal/Mol-Kelvin & Cal/Mol-Kelvin \\
Total & 163.817 & 41.465 & 94.950
\end{tabular}

\begin{tabular}{|l|l|}
\hline$\alpha$-Fenchyl cation $\left(\alpha-\mathrm{F}^{+}\right)$ & \\
\hline &
\end{tabular}

\section{optimized xyz-matrix}

C 0.51635300000 .39332400001 .1412620000

C $1.0801480000-0.3787030000-0.0846170000$

C $-0.1952740000-0.2229840000-0.9376340000$

C $-1.3217420000-0.3882580000-0.0957690000$

C $-0.51073100001 .4268170000-0.7372060000$

C 0.19682300001 .78819000000 .5762560000

H 1.09603700002 .37849400000 .3972960000

H - 0.45366100002 .36036100001 .2407760000

H - $1.55940200001 .7315360000-0.7973280000$

H $-0.03288900001 .7886330000-1.6524500000$

C $-0.8779850000-0.23692800001 .3240160000$ 
H - 1.58112900000 .33607500001 .9360770000

H - $0.8266010000-1.24904100001 .7502130000$

C - $2.7081450000-0.6709560000-0.5203570000$

C $1.3818090000-1.85706500000 .1928720000$

C $2.31606900000 .2552910000-0.7261900000$

H 1.14434900000 .37775500002 .0327330000

H - $2.8803890000-1.7357900000-0.3015070000$

H - $2.8602490000-0.5213090000-1.5906200000$

H -3.4402300000 - 0.10751700000 .0640560000

H 2.6500150000 - $0.3491720000-1.5743190000$

H $3.13445500000 .2854850000-0.0004610000$

H 2.1541180000 1.2723010000 -1.0900280000

H $2.2194890000-1.93619200000 .8917940000$

H $1.6742880000-2.3635740000-0.7311770000$

H $0.5375820000-2.40858200000 .6154790000$

H - $0.2397400000-0.5118300000-1.9863390000$

\section{Thermodynamic data}

Zero-point correction $=0.249954$ (Hartree/Particle)

Thermal correction to Energy $=0.260320$

Thermal correction to Enthalpy $=0.261265$

Thermal correction to Gibbs Free Energy $=0.215454$

Sum of electronic and zero-point Energies $=-390.580465$

Sum of electronic and thermal Energies $=-390.570098$

Sum of electronic and thermal Enthalpies $=-390.569154$

Sum of electronic and thermal Free Energies $=-\mathbf{3 9 0 . 6 1 4 9 6 4}$

\begin{tabular}{rlrr} 
& E (Thermal) & CV & \multicolumn{1}{c}{ S } \\
KCal/Mol & Cal/Mol-Kelvin & Cal/Mol-Kelvin \\
Total & 163.354 & 41.578 & 96.416
\end{tabular}

\begin{tabular}{|l|l|l|}
\hline Phellandryl cation $\left(\mathrm{Ph}^{+}\right)$ & 1 \\
\hline
\end{tabular}

optimized xyz-matrix

C $1.4844250000-1.1960680000-0.3078700000$

C $0.1594130000-1.1358290000-0.6787860000$

C $-0.69346100000 .0712830000-0.5994550000$

C -0.06827800001 .13292500000 .3082680000$

C 1.42796400001 .28169600000 .0389470000

C $2.1561030000-0.01439700000 .0234400000$

C $3.6050470000-0.01458200000 .3098630000$

H 1.91145200001 .97225800000 .7382810000

H $1.60080200001 .7154020000-0.9640700000$

H -0.21564300000 .84682700001 .3560140000$

H -0.56184400002 .09589800000 .1626130000$

C - $2.1578080000-0.3138320000-0.2461050000$

H $-0.71656000000 .4534860000-1.6413470000$

H - $0.2996880000-2.0368020000-1.0900120000$

H $2.0343920000-2.1296070000-0.3717210000$ 
H $4.1043540000-0.93747300000 .0137000000$

H 3.71748800000 .10949200001 .3993530000

H $4.09527600000 .8551360000-0.1409400000$

C - $3.06343700000 .9160750000-0.2683100000$

C - $2.2504220000-1.04601800001 .0948170000$

H - $2.4903080000-1.0001800000-1.0364740000$

H -2.8265500000 1.59963300000 .5536920000

H -4.1060450000 $0.6111200000-0.1464790000$

H - 2.9813640000 $1.4653670000-1.2116440000$

H -1.6107340000 -1.9364660000 1.1297260000

H -3.2779420000 -1.3755070000 1.2690390000

H -1.9743690000 -0.3961110000 1.9314170000

\section{Thermodynamic data}

Zero-point correction $=0.249033$ (Hartree/Particle)

Thermal correction to Energy $=0.260243$

Thermal correction to Enthalpy $=0.261187$

Thermal correction to Gibbs Free Energy $=0.212494$

Sum of electronic and zero-point Energies $=-390.582219$

Sum of electronic and thermal Energies $=-390.571009$

Sum of electronic and thermal Enthalpies $=-390.570065$

Sum of electronic and thermal Free Energies $=-\mathbf{3 9 0 . 6 1 8 7 5 8}$

\begin{tabular}{rlrr} 
& E (Thermal) & CV & \multicolumn{1}{c}{ S } \\
KCal/Mol & Cal/Mol-Kelvin & Cal/Mol-Kelvin \\
Total & 163.305 & 42.475 & 102.484
\end{tabular}

\begin{tabular}{|l|l|}
\hline Terpinyl cation $\left(\mathbf{T}^{+}\right)$ & \\
\hline
\end{tabular}

optimized xyz-matrix

C 0.96148200000 .26409800000 .9217740000

C - 0.05930800001 .40076700001 .0429200000

C - 1.36372500001 .12567900000 .3439210000

C $-1.7758890000-0.0722230000-0.0832150000$

C $-0.9488080000-1.31842000000 .1192570000$

C $0.2106910000-1.13665200001 .0867130000$

H - $0.1619350000-1.08135200002 .1142900000$

H $0.9211150000-1.96631000001 .0386050000$

H - $0.6136510000-1.7101100000-0.8528260000$

H -1.5936910000 -2.1097750000 0.5231280000

C $1.69094200000 .1493090000-0.3305620000$

C $3.0149880000-0.4915630000-0.3245410000$

C $1.15483300000 .6037600000-1.6173020000$

C - $3.0891680000-0.2713490000-0.7851450000$

H 0.37972900002 .33505000000 .6712180000

H - 0.23294600001 .56743700002 .1136420000

H 1.69288300000 .28221100001 .7387890000

H -3.7454500000 -0.9227780000 -0.1974730000 
H - $2.9443870000-0.7585300000-1.7567080000$

H -3.6047810000 $0.6769140000-0.9493620000$

H $1.94894200000 .8171700000-2.3379110000$

H $0.43140400001 .4153510000-1.5314750000$

H $0.6006110000-0.2660630000-2.0199580000$

H $3.73236700000 .3502380000-0.3130910000$

H $3.2183610000-1.0463580000-1.2450660000$

H $3.2043420000-1.09225300000 .5669090000$

H - 2.00913800001 .98872500000 .1943670000

\section{Thermodynamic data}

Zero-point correction $=0.248012$ (Hartree/Particle)

Thermal correction to Energy $=0.259243$

Thermal correction to Enthalpy $=0.260187$

Thermal correction to Gibbs Free Energy= 0.211832

Sum of electronic and zero-point Energies $=-390.563997$

Sum of electronic and thermal Energies $=-390.552766$

Sum of electronic and thermal Enthalpies $=-390.551822$

Sum of electronic and thermal Free Energies $=-390.600177$

\begin{tabular}{ccrr} 
& E (Thermal) & CV & \multicolumn{1}{c}{ S } \\
& KCal/Mol & Cal/Mol-Kelvin & Cal/Mol-Kelvin \\
Total & 162.677 & 43.135 & 101.773
\end{tabular}

\begin{tabular}{|l|l|}
\hline Norbornyl endo diazonium (endo- $\left.\mathbf{N}-\mathrm{N}_{2}{ }^{+}\right)$ &
\end{tabular}

\section{optimized xyz-matrix}

C $1.4275220000-0.5724850000-0.5970320000$

C $0.0883630000-1.3224590000-0.7154570000$

C $-0.7031830000-0.72456000000 .4971710000$

C 0.24043400000 .33997200001 .1164160000

C 0.41498000001 .51171400000 .1332310000

C $1.22096300000 .8859030000-1.0444250000$

H $0.69672900000 .9534520000-2.0018610000$

H $2.18219700001 .3893780000-1.1665150000$

H 0.98350900002 .29924400000 .6326100000

H $-0.52988600001 .9698300000-0.1778570000$

C $1.5650520000-0.43117500000 .9285960000$

H 2.41711300000 .18051400001 .2354610000

H $1.6022820000-1.38616300001 .4618520000$

H -0.04339200000 .61005100002 .1339920000$

H $0.1917230000-2.3977460000-0.5551020000$

H - $0.4174290000-1.1666850000-1.6721480000$

H 2.2388680000 -1.0937940000 -1.1058090000

$\mathrm{N}-1.9549390000-0.10408000000 .0390310000$

N -2.8719420000 $0.3884410000-0.3119780000$

H -1.0583380000 -1.4700690000 1.2150000000 
Thermodynamic Data

Zero-point correction $=0.178008$ (Hartree/Particle)

Thermal correction to Energy $=0.185415$

Thermal correction to Enthalpy $=0.186359$

Thermal correction to Gibbs Free Energy $=0.146131$

Sum of electronic and zero-point Energies $=-\mathbf{3 8 2 . 2 2 4 6 7 5}$

Sum of electronic and thermal Energies $=-382.217268$

Sum of electronic and thermal Enthalpies $=-382.216324$

Sum of electronic and thermal Free Energies $=-\mathbf{3 8 2 . 2 5 6 5 5 3}$

$\mathrm{E}$ (Thermal) CV S

$\mathrm{KCal} / \mathrm{Mol} \quad \mathrm{Cal} / \mathrm{Mol}-K e l v i n \quad \mathrm{Cal} / \mathrm{Mol}-K e l v i n$

$\begin{array}{lll}\text { Total } & 116.350 & 29.801\end{array}$

\begin{tabular}{|l|l|l|}
\hline Norbornyl exo diazonium $\left(\boldsymbol{e x o}-\mathbf{N}-\mathbf{N}_{2}{ }^{+}\right)$ & \\
\hline
\end{tabular}

optimized xyz-matrix

C - 1.07792000001 .03538700000 .4215640000

C $0.08831400001 .2866050000-0.5584610000$

C $0.7053380000-0.1426760000-0.6916350000$

C - $0.1334290000-1.01957100000 .2695100000$

C $-1.4870290000-1.1813890000-0.4635450000$

C - $2.15560400000 .2158740000-0.3090530000$

H - 2.4344040000 $0.6595900000-1.2680490000$

H -3.0611470000 0.15087000000 .2979830000

H -2.0598570000 -1.9629350000 0.0401510000

H - $1.3657430000-1.4943440000-1.5046860000$

C $-0.4733000000-0.00784900001 .3756840000$

H - $1.1996260000-0.41329000002 .0840420000$

H 0.39613700000 .35361100001 .9373710000

H $0.3496460000-1.95796500000 .5472650000$

H $0.81399200001 .9946810000-0.1487600000$

H - $0.22272900001 .6496320000-1.5400370000$

H - 1.43668600001 .95744500000 .8798500000

N $2.1055290000-0.1138200000-0.1980970000$

N $3.1232190000-0.10360000000 .2147820000$

H $0.8209610000-0.5336470000-1.7063040000$

\section{Thermodynamic Data}

Zero-point correction $=0.177614$ (Hartree/Particle)

Thermal correction to Energy $=0.185112$

Thermal correction to Enthalpy $=0.186056$

Thermal correction to Gibbs Free Energy $=0.145632$

Sum of electronic and zero-point Energies $=-382.225583$

Sum of electronic and thermal Energies $=-382.218085$

Sum of electronic and thermal Enthalpies $=-382.217141$

Sum of electronic and thermal Free Energies $=-382.257565$ 


\begin{tabular}{llrr} 
& E (Thermal) & CV & \multicolumn{1}{c}{ S } \\
KCal/Mol & Cal/Mol-Kelvin & Cal/Mol-Kelvin \\
Total & 116.159 & 29.992 & 85.079
\end{tabular}

\begin{tabular}{|l|l|}
\hline endo bornane diazonium $\left(\right.$ endo-B- $\left.\mathbf{N}_{2}{ }^{+}\right)$ & \\
\hline
\end{tabular}

optimized xyz-matrix

C - 0.74580700001 .40969600000 .2600520000

C 0.51111900001 .29117500001 .1441590000

C $0.9766370000-0.16051900000 .8041460000$

C $0.0177330000-0.6645240000-0.3215510000$

C $0.27600400000 .2049030000-1.5724600000$

C - $0.26836000001 .6104510000-1.1893130000$

H $0.49163900002 .3928880000-1.2638420000$

H -1.0927560000 $1.9062020000-1.8405170000$

H - $0.2635640000-0.2264920000-2.4186860000$

H $1.32961200000 .2147030000-1.8745210000$

C - $1.3041730000-0.03807700000 .2527190000$

C - $2.4951170000-0.2415320000-0.6889670000$

C - $1.7180740000-0.56422400001 .6324610000$

C $0.0702670000-2.1634880000-0.5373450000$

H 0.30481600001 .35371900002 .2134390000

H 1.26862400002 .04151700000 .9027600000

H - 1.43714000002 .17335900000 .6234200000

N 2.3614660000 -0.1701820000 0.3341120000

N $3.3853110000-0.1833230000-0.0652300000$

H $1.0389970000-2.4828940000-0.9384890000$

H $-0.6896730000-2.4568660000-1.2671300000$

H - $0.1171450000-2.71515500000 .3896390000$

H - $1.9951690000-1.62143900001 .5741820000$

H - 2.5999000000 -0.0170760000 1.9788160000

H $-0.9582970000-0.46297200002 .4137820000$

H -3.3440410000 $0.3485620000-0.3302310000$

H - 2.8051990000 -1.2911180000 -0.6896400000

H - $2.29972100000 .0528680000-1.7211730000$

H $1.0201010000-0.83843100001 .6626040000$

\section{Thermodynamic Data}

Zero-point correction $=0.262349$ (Hartree/Particle)

Thermal correction to Energy $=0.274059$

Thermal correction to Enthalpy $=0.275003$

Thermal correction to Gibbs Free Energy $=0.226198$

Sum of electronic and zero-point Energies $=-500.030324$

Sum of electronic and thermal Energies $=-500.018614$

Sum of electronic and thermal Enthalpies $=-500.017670$ 
Sum of electronic and thermal Free Energies $=-500.066475$

\begin{tabular}{llrr} 
& E (Thermal) & CV & \multicolumn{1}{c}{ S } \\
KCal/Mol & Cal/Mol-Kelvin & Cal/Mol-Kelvin \\
Total & 171.975 & 47.508 & 102.719
\end{tabular}

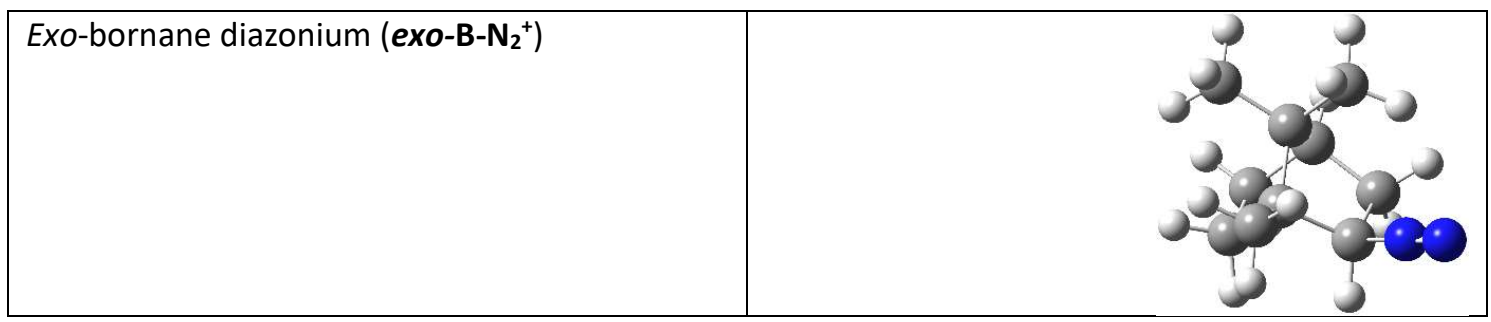

\section{optimized xyz-matrix}

C - 1.05826500000 .76413000001 .0250230000

C 0.28391000001 .52774700000 .9814800000

C $0.93192500000 .9415500000-0.3085500000$

C $-0.0595070000-0.1556590000-0.8035660000$

C $-1.20130300000 .7278290000-1.3838040000$

C - $1.91860800001 .3036280000-0.1312180000$

H - $1.96070100002 .3955470000-0.1354130000$

H - $2.94614200000 .9424560000-0.0600070000$

$\mathrm{H}-1.86048900000 .0971500000-1.9847960000$

H $-0.81775400001 .5005400000-2.0580990000$

C $-0.6733770000-0.65797300000 .5418830000$

C - $1.8701270000-1.58924600000 .3168050000$

C $0.2973280000-1.39524200001 .4733040000$

C $0.5126050000-1.1700040000-1.7738230000$

H 0.90239700001 .34585500001 .8626350000

H 0.16498800002 .60778800000 .8761700000

H - 1.52961600000 .81430000002 .0092050000

N 2.2517820000 0.3432490000 -0.0132730000

N $3.2294480000-0.12743500000 .1584210000$

H $0.9149970000-0.6885590000-2.6715480000$

H - $0.2828140000-1.8478640000-2.0951330000$

H $1.3009140000-1.7847920000-1.3245380000$

H $0.7304770000-2.27186000000 .9788250000$

H - $0.2493910000-1.76198600002 .3468300000$

H $1.1145600000-0.78489200001 .8691560000$

H - 2.4011870000 -1.7386510000 1.2616460000

H - $1.5262020000-2.5713170000-0.0235630000$

H - 2.5902410000 - $1.2163180000-0.4132290000$

H $1.20011400001 .6713440000-1.0793830000$

\section{Thermodynamic Data}

Zero-point correction $=0.262577$ (Hartree/Particle)

Thermal correction to Energy $=0.274165$

Thermal correction to Enthalpy $=0.275109$

Thermal correction to Gibbs Free Energy $=0.226700$

Sum of electronic and zero-point Energies $=-500.029968$

Sum of electronic and thermal Energies $=-500.018380$ 
Sum of electronic and thermal Enthalpies $=-500.017436$

Sum of electronic and thermal Free Energies $=-\mathbf{5 0 0 . 0 6 5 8 4 5}$

\begin{tabular}{llrr} 
& E (Thermal) & CV & \multicolumn{1}{c}{ S } \\
& KCal/Mol & Cal/Mol-Kelvin & Cal/Mol-Kelvin \\
Total & 172.041 & 47.413 & 101.885
\end{tabular}

\begin{tabular}{|l|l|}
\hline fenchane endo-diazonium (endo-F- $\left.\mathbf{N}_{2}{ }^{+}\right)$ & (
\end{tabular}

optimized xyz-matrix

C - $0.5711440000-1.5150110000-0.2183280000$

C - $1.2572270000-0.18596000000 .2183630000$

C 0.03225200000 .62294400000 .6701050000

C $1.2654730000-0.24681500000 .2825710000$

C $1.2983480000-0.3933580000-1.2573310000$

C $0.1248130000-1.3590070000-1.5798060000$

H - $0.5311120000-1.0001170000-2.3741970000$

H $0.5087160000-2.3304110000-1.9006050000$

H 2.2628580000 -0.8192100000 -1.5463980000

H $1.22528500000 .5695160000-1.7785150000$

C $0.6501460000-1.59664800000 .7144150000$

H $1.3221140000-2.42345600000 .4668340000$

H $0.4178760000-1.64567700001 .7828310000$

C 2.55989400000 .19735600000 .9348310000

C - $2.1511330000-0.39341900001 .4443800000$

C - $2.06262400000 .5046790000-0.8832590000$

H - $1.2722530000-2.3514650000-0.1659990000$

N 0.10813500001 .92872900000 .0316520000

N $0.18307200002 .8927650000-0.4914620000$

H 2.45926700000 .27510400002 .0220830000

H 2.90159000001 .16383100000 .5468430000

H $3.3454260000-0.53272400000 .7216880000$

H - 2.5904090000 $1.3831250000-0.4961400000$

H - $2.8199500000-0.1902530000-1.2569140000$

H - $1.45563800000 .8118800000-1.7406480000$

H -3.0237680000 -0.9874140000 1.1586580000

H -2.5129710000 0.56241000001 .8374970000

H - $1.6349310000-0.92382800002 .2496290000$

H 0.02666600000 .87967300001 .7363820000

\section{Thermodynamic Data}

Zero-point correction $=0.262115$ (Hartree/Particle)

Thermal correction to Energy $=0.273728$

Thermal correction to Enthalpy $=0.274672$

Thermal correction to Gibbs Free Energy $=0.226109$

Sum of electronic and zero-point Energies $=-500.033458$

Sum of electronic and thermal Energies $=-500.021845$

Sum of electronic and thermal Enthalpies $=-500.020901$ 
Sum of electronic and thermal Free Energies $=-500.069464$

\begin{tabular}{llrr} 
& E (Thermal) & CV & \multicolumn{1}{c}{ S } \\
& KCal/Mol & Cal/Mol-Kelvin & Cal/Mol-Kelvin \\
Total & 171.767 & 47.454 & 102.210
\end{tabular}

\begin{tabular}{|l|l|l|}
\hline fenchane exo-diazonium $\left(\right.$ exo-F-N $\left.{ }^{+}\right)$ & \\
\hline
\end{tabular}

11

C - $1.2203090000-0.39927000000 .9091130000$

C $-0.94589200000 .8145140000-0.0331330000$

C $0.42392900000 .3480990000-0.6806440000$

C $0.7606310000-1.0269000000-0.0280380000$

C - $0.2956780000-1.9773210000-0.6582510000$

C - $1.6101970000-1.64202900000 .0943620000$

H - $2.4622650000-1.4841440000-0.5682140000$

$\mathrm{H}-1.8802780000-2.45189600000 .7763950000$

H $0.0226080000-3.0086110000-0.4838530000$

$\mathrm{H}-0.3689360000-1.8481190000-1.7438550000$

C $0.1872840000-0.79722500001 .3820670000$

H $0.1937800000-1.71769800001 .9723010000$

H $0.7191600000-0.02520400001 .9505710000$

C 2.1986140000 -1.4876710000 -0.1731550000

C -0.82867800002 .10652200000 .7837410000$

C - $1.97489700001 .0172010000-1.1473280000$

H - $1.9341110000-0.13288300001 .6922020000$

N $1.49748800001 .2948890000-0.3403270000$

N 2.3032410000 $1.9762430000-0.0335360000$

H $2.9062190000-0.81790200000 .3284260000$

H $2.4884750000-1.5806820000-1.2251940000$

H 2.3092830000 -2.4722020000 0.2885950000

H - $1.76975700001 .9390120000-1.7007190000$

H - 2.9742740000 $1.1036580000-0.7103240000$

H -1.9898390000 $0.1892820000-1.8615500000$

H - 1.81696700002 .35330200001 .1799310000

H -0.51080300002 .95444200000 .1666930000$

H - 0.15206500002 .01323500001 .6392710000

H $0.44582000000 .3529680000-1.7760410000$

\section{Thermodynamic Data}

Zero-point correction $=0.261982$ (Hartree/Particle)

Thermal correction to Energy $=0.273575$

Thermal correction to Enthalpy $=0.274519$

Thermal correction to Gibbs Free Energy $=0.226108$

Sum of electronic and zero-point Energies $=-500.034071$

Sum of electronic and thermal Energies $=-500.022477$

Sum of electronic and thermal Enthalpies $=-500.021533$

Sum of electronic and thermal Free Energies $=-500.069945$ 


\begin{tabular}{llrr} 
& E (Thermal) & CV & \multicolumn{1}{c}{ S } \\
& KCal/Mol & Cal/Mol-Kelvin & Cal/Mol-Kelvin \\
Total & 171.671 & 47.507 & 101.890
\end{tabular}

\begin{tabular}{|l|l|}
\hline $\begin{array}{l}\text { Norbornyl endo diazonium dissociation TS } \\
\text { (endo- } \mathrm{N}_{\mathbf{N}}{ }^{+} \text {-TS) }\end{array}$ \\
\hline
\end{tabular}

\section{optimized xyz-matrix}

C $1.4509260000-0.4139800000-0.7470500000$

C $0.2033520000-1.3152320000-0.7258120000$

C $-0.4501510000-0.87297500000 .5685400000$

C 0.35931300000 .21098400001 .1756510000

C 0.33809200001 .47878900000 .2828380000

C $1.01698700001 .0335410000-1.0418760000$

H $0.34407800001 .0918060000-1.9018750000$

H $1.88214400001 .6604360000-1.2661020000$

H 0.92089200002 .24806300000 .7939150000

H -0.67123600001 .87746800000 .1533790000$

C $1.7608290000-0.37528200000 .7606020000$

H 2.54042700000 .33351100001 .0517990000

H $1.9888530000-1.35354300001 .1932380000$

H $0.4687530000-2.3724910000-0.5966680000$

H - $0.4467540000-1.2537820000-1.6030180000$

H 2.2429470000 -0.7953560000 -1.3908690000

N -2.1179330000 -0.0954210000 0.0449150000

$\mathrm{N}-3.10260100000 .2817160000-0.2588590000$

H - $0.9790020000-1.59448100001 .1905580000$

H 0.17654900000 .37922700002 .2358840000

Thermodynamic Data

Zero-point correction $=0.174913$ (Hartree/Particle)

Thermal correction to Energy $=0.182727$

Thermal correction to Enthalpy $=0.183671$

Thermal correction to Gibbs Free Energy= 0.141894

Sum of electronic and zero-point Energies $=-382.219833$

Sum of electronic and thermal Energies $=-382.212019$

Sum of electronic and thermal Enthalpies $=-\mathbf{3 8 2 . 2 1 1 0 7 5}$

Sum of electronic and thermal Free Energies $=-382.252852$
$E$ (Thermal)
CV
$\mathrm{S}$
$\mathrm{KCal} / \mathrm{Mol} \quad \mathrm{Cal} / \mathrm{Mol}-\mathrm{Kelvin}$
Cal/Mol-Kelvin

Total 114.663

29.896

87.928 


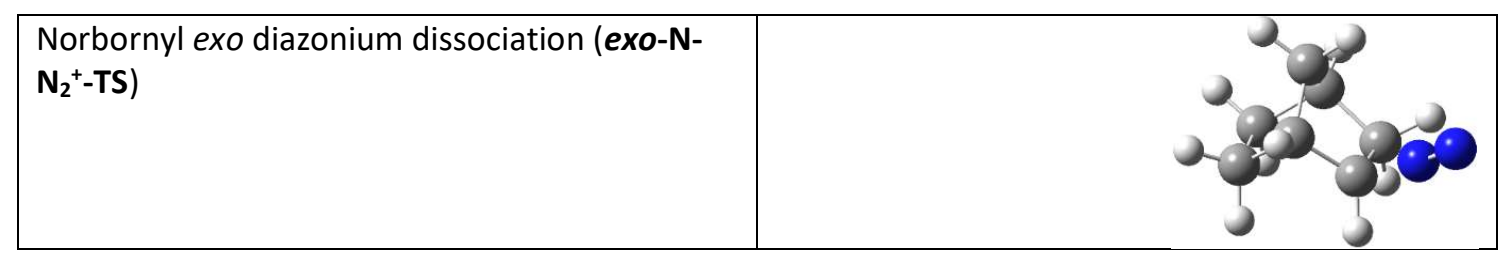

optimized xyz-matrix

C $1.0836790000-1.03793900000 .4382440000$

C - $0.0044860000-1.2239730000-0.6497650000$

C $-0.56436600000 .1861910000-0.7667160000$

C 0.14205000001 .02063800000 .2450140000

C $1.55225700001 .1477270000-0.4486940000$

C $2.2253700000-0.2258940000-0.1898060000$

H $2.6132210000-0.6822280000-1.1035200000$

H $3.0561850000-0.12705500000 .5121620000$

H 2.07217900001 .97165900000 .0450500000

H $1.48351600001 .4081320000-1.5095560000$

C 0.42916900000 .00257500001 .3630700000

H 1.11671800000 .39995800002 .1129430000

H - $0.4747790000-0.35544300001 .8685350000$

$\mathrm{H}-0.31592700001 .98063100000 .4818650000$

H - $0.7686170000-1.9396430000-0.3328870000$

H $0.3718270000-1.5609330000-1.6202460000$

H $1.3817440000-1.97801200000 .9021300000$

N -2.2703930000 $0.0875230000-0.1795420000$

$\mathrm{N}-3.28349700000 .06412400000 .2408310000$

H - $0.84086500000 .6054560000-1.7335860000$

\section{Thermodynamic Data}

Zero-point correction $=0.175335$ (Hartree/Particle)

Thermal correction to Energy $=0.183039$

Thermal correction to Enthalpy $=0.183983$

Thermal correction to Gibbs Free Energy $=0.142790$

Sum of electronic and zero-point Energies $=-382.223880$

Sum of electronic and thermal Energies $=-382.216176$

Sum of electronic and thermal Enthalpies $=-\mathbf{3 8 2 . 2 1 5 2 3 2}$

Sum of electronic and thermal Free Energies $=-\mathbf{3 8 2 . 2 5 6 4 2 5}$

\begin{tabular}{rlrr} 
& E (Thermal) & CV & \multicolumn{1}{c}{ S } \\
KCal/Mol & Cal/Mol-Kelvin & Cal/Mol-Kelvin \\
Total & 114.859 & 29.678 & 86.698
\end{tabular}

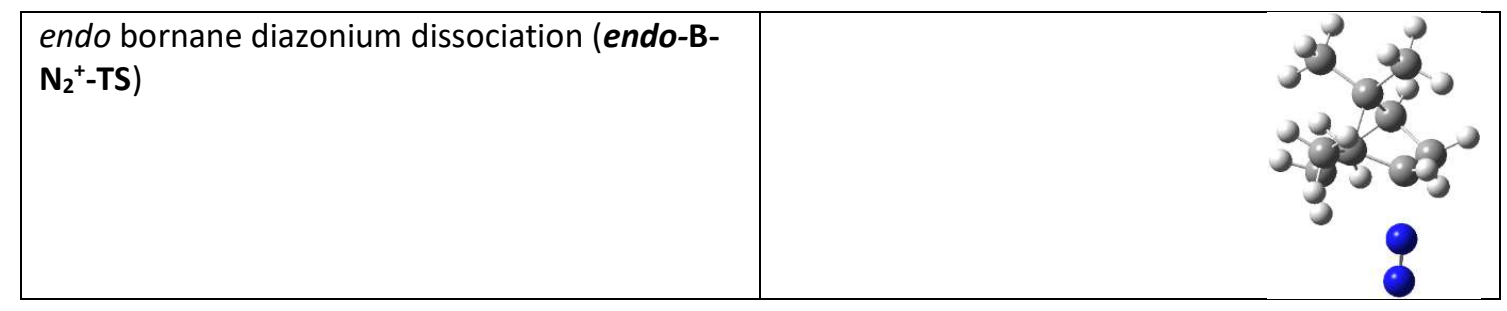

optimized xyz-matrix

C $0.8224760000-1.41742900000 .2390330000$ 
C - $0.4345060000-1.29213100001 .1310350000$

C -0.81187300000 .15058500000 .8409630000$

C $-0.02000700000 .6693430000-0.2975260000$

C $-0.2743380000-0.2176570000-1.5438810000$

C $0.3372060000-1.6039780000-1.2053510000$

H - $0.3889660000-2.4160940000-1.2899780000$

H $1.1664180000-1.8457290000-1.8726630000$

H $0.20874500000 .2479420000-2.4057830000$

H - $1.3419140000-0.2659610000-1.7824310000$

C 1.36338500000 .04001200000 .2514950000

C $2.50651000000 .3129260000-0.7263610000$

C 1.80596700000 .54947900001 .6303290000

C - $0.07995400002 .1654660000-0.5236560000$

H - $0.2381970000-1.40724600002 .1995500000$

H - $1.2184420000-2.00672000000 .8650930000$

H $1.5215360000-2.17876300000 .5908550000$

$\mathrm{N}-2.61306100000 .13362900000 .2930510000$

N $-3.66619000000 .1609630000-0.0136840000$

$\mathrm{H}-1.06195800002 .4670230000-0.9044980000$

H $0.66146900002 .4618030000-1.2704660000$

H 0.11763600002 .72493000000 .3961880000

H 2.00258800001 .62553600001 .5960260000

H 2.74211700000 .05756100001 .9101290000

H 1.09511700000 .36700500002 .4420650000

H $3.3713350000-0.2889960000-0.4274610000$

H $2.80779400001 .3636890000-0.6810870000$

H 2.2732940000 0.0701840000 - 1.7637420000

H -1.0530080000 0.82200800001 .6661470000

\section{Thermodynamic Data}

Zero-point correction $=0.259645$ (Hartree/Particle)

Thermal correction to Energy $=0.271748$

Thermal correction to Enthalpy $=0.272692$

Thermal correction to Gibbs Free Energy $=0.222656$

Sum of electronic and zero-point Energies $=-500.025690$

Sum of electronic and thermal Energies $=-500.013587$

Sum of electronic and thermal Enthalpies $=-500.012643$

Sum of electronic and thermal Free Energies $=-500.062679$

\begin{tabular}{llrr} 
& E (Thermal) & CV & \multicolumn{1}{c}{ S } \\
& KCal/Mol & Cal/Mol-Kelvin & Cal/Mol-Kelvin \\
Total & 170.524 & 47.622 & 105.310
\end{tabular}

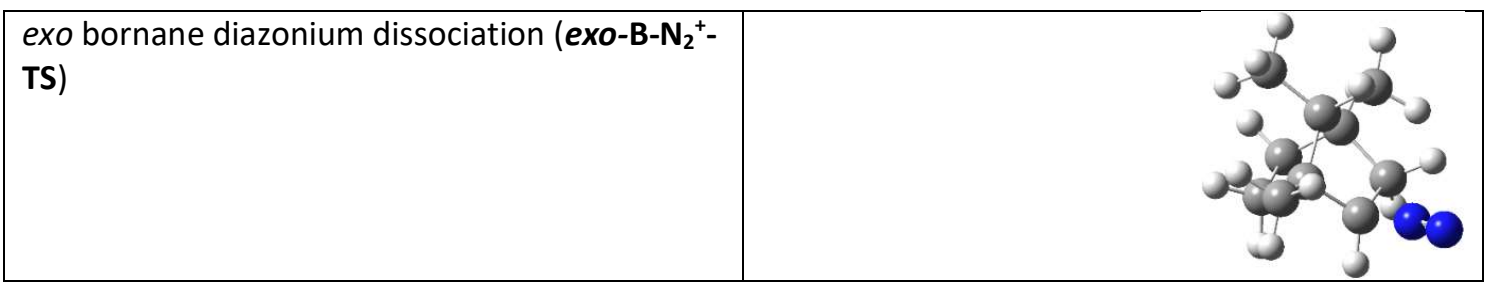

optimized xyz-matrix

C - $1.1037620000-0.6652540000-1.0797640000$ 
C $0.1876860000-1.5201650000-0.9930440000$ C $0.7848510000-1.02248900000 .3158980000$ C - 0.07394400000 .10024300000 .8068460000 C - $1.2877480000-0.78726700001 .3053800000$ C - $2.0395320000-1.19863400000 .0148490000$ H - $2.1852910000-2.2789630000-0.0579290000$ H -3.0250560000 - $0.7332570000-0.0408840000$ H - $1.8872980000-0.15551500001 .9654360000$ H - $0.9632610000-1.64004100001 .9112580000$ C - $0.64768500000 .7061620000-0.5157360000$ C - $1.78820700001 .6946310000-0.2520880000$ C $0.38041400001 .4259650000-1.3977610000$ C 0.50330100001 .01377200001 .8676670000 H $0.8552400000-1.3502670000-1.8405820000$ H $0.0050650000-2.5969600000-0.9388730000$ H - $1.5265640000-0.6435110000-2.0861850000$ N 2.3869700000 - $0.3479500000-0.0339210000$ N $3.39230700000 .0748430000-0.1520160000$ H 0.86140800000 .44913400002 .7348380000 H - 0.26656300001 .70636700002 .2176550000 H 1.33415900001 .61250900001 .4776680000 H $0.88733900002 .2246010000-0.8446720000$ H - $0.13505500001 .9001710000-2.2378640000$ H $1.14129600000 .7755380000-1.8389350000$ H - 2.3267830000 $1.8935790000-1.1836120000$ $\mathrm{H}-1.38434200002 .64858900000 .1017880000$ $\mathrm{H}-2.51478100001 .34815700000 .4852520000$ H $1.1833100000-1.73017700001 .0437170000$

\section{Thermodynamic Data}

Zero-point correction $=0.260782$ (Hartree/Particle) Thermal correction to Energy $=0.272351$

Thermal correction to Enthalpy $=0.273295$

Thermal correction to Gibbs Free Energy $=0.224866$ Sum of electronic and zero-point Energies $=-500.028262$ Sum of electronic and thermal Energies $=-500.016692$ Sum of electronic and thermal Enthalpies $=-500.015748$ Sum of electronic and thermal Free Energies $=-500.064177$

\begin{tabular}{llrr} 
& E (Thermal) & CV & \multicolumn{1}{c}{ S } \\
& KCal/Mol & Cal/Mol-Kelvin & Cal/Mol-Kelvin \\
Total & 170.903 & 46.835 & 101.928
\end{tabular}

\begin{tabular}{|l|l|}
\hline $\begin{array}{l}\text { fenchane endo-diazonium dissociation (endo-F- } \\
\mathrm{N}^{+}{ }^{-} \text {-TS) }\end{array}$ & \\
\hline
\end{tabular}

optimized xyz-matrix

C $1.0182900000-1.31768900000 .3289160000$

C $1.26935800000 .1249970000-0.2141350000$ 
C - $0.13908300000 .3934550000-0.7500790000$

C - $1.1030330000-0.6349190000-0.2969160000$

C - $1.1237460000-0.71044700001 .2530640000$

C $0.2595560000-1.28277300001 .6599390000$

H $0.7591190000-0.70038100002 .4356240000$

H $0.1545280000-2.30080500002 .0431360000$

H -1.9418770000 -1.3707110000 1.5517340000

H - 1.33307100000 .27054000001 .6941050000

C - $0.0882390000-1.8036580000-0.6271760000$

H - $0.5553760000-2.7510390000-0.3402300000$

H $0.1988550000-1.8652680000-1.6810570000$

C - $2.4516660000-0.6438700000-0.9867870000$

C $2.24364100000 .1113640000-1.4044080000$

C 1.77264600001 .12889000000 .8294350000

H $1.9352590000-1.91029600000 .3457290000$

$\mathrm{N}-0.76321500002 .0389040000-0.0152160000$

$\mathrm{N}-1.20424600002 .98028500000 .3357260000$

H - $2.3498680000-0.6133980000-2.0758750000$

H $-3.06008300000 .2108970000-0.6715370000$

H -3.0001220000 -1.5515600000 -0.7225920000

H 1.98596800002 .09797300000 .3676800000

H 2.70560400000 .75172000001 .2572680000

H 1.06417500001 .27945200001 .6482910000

H $3.2422600000-0.1480270000-1.0421430000$

H $2.30096800001 .0980670000-1.8749390000$

H $1.9672760000-0.6200700000-2.1692010000$

H $-0.24773100000 .7964800000-1.7606840000$

\section{Thermodynamic Data}

Zero-point correction $=0.259860$ (Hartree/Particle)

Thermal correction to Energy $=0.271831$

Thermal correction to Enthalpy $=0.272775$

Thermal correction to Gibbs Free Energy $=0.223082$

Sum of electronic and zero-point Energies $=-500.027831$

Sum of electronic and thermal Energies $=-500.015859$

Sum of electronic and thermal Enthalpies $=-500.014915$

Sum of electronic and thermal Free Energies $=-500.064608$

\begin{tabular}{llrr} 
& E (Thermal) & CV & \multicolumn{1}{c}{ S } \\
& KCal/Mol & Cal/Mol-Kelvin & Cal/Mol-Kelvin \\
Total & 170.577 & 47.353 & 104.587
\end{tabular}

\begin{tabular}{|l|l|}
\hline $\begin{array}{l}\text { fenchane exo-diazonium dissociation (exo-F- } \\
\mathbf{N}_{2}{ }^{+} \text {-TS) }\end{array}$ &
\end{tabular}

optimized xyz-matrix

C $1.2614410000-0.30939500000 .9436790000$

C $0.4067710000-1.1682230000-0.0522110000$

C $-0.4307650000-0.0536610000-0.6927990000$

C $-0.08490800001 .2488720000-0.0388890000$ 
C $1.35322200001 .4738620000-0.6536710000$ C 2.27506800000 .53234300000 .1567110000 H $2.9458680000-0.0588620000-0.4681150000$ H 2.89671000001 .10364700000 .8501870000 H $1.59353700002 .5301600000-0.5054950000$ H $1.38267400001 .2936800000-1.7343470000$ C 0.26616800000 .77573200001 .3862680000 H 0.73356100001 .57013600001 .9742140000 H - 0.60455500000 .40563700001 .9392350000 C - $1.03212500002 .4149520000-0.2402360000$ C - $0.4110040000-2.21248600000 .7240450000$ C $1.2055560000-1.8859140000-1.1501930000$ H $1.6921130000-0.92618200001 .7353290000$ N - $2.1472470000-0.3335440000-0.2696160000$ $\mathrm{N}-3.2105610000-0.4095260000-0.0110530000$ H - 2.0002700000 2.2397100000 0.2404900000 H - $1.20082200002 .6181320000-1.3027260000$ H -0.60414400003 .31445700000 .2092450000$ H $0.5557720000-2.5525660000-1.7249970000$ H $1.9900080000-2.4927780000-0.6884000000$ H $1.6847390000-1.1943780000-1.8488400000$ H $0.2770680000-2.96613400001 .1147090000$ H - $1.1257200000-2.72543200000 .0719920000$ H - $0.9458270000-1.77975600001 .5743630000$ H - $0.6226080000-0.0744690000-1.7683780000$

\section{Thermodynamic Data}

Zero-point correction $=0.260224$ (Hartree/Particle) Thermal correction to Energy $=0.272002$

Thermal correction to Enthalpy $=0.272946$

Thermal correction to Gibbs Free Energy $=0.223764$ Sum of electronic and zero-point Energies $=-500.031791$ Sum of electronic and thermal Energies $=-500.020013$ Sum of electronic and thermal Enthalpies $=-500.019069$ Sum of electronic and thermal Free Energies $=-500.068250$

$\begin{array}{llrr} & \text { E (Thermal) } & \text { CV } & \text { S } \\ \text { KCal/Mol } & \text { Cal/Mol-Kelvin } & \text { Cal/Mol-Kelvin } \\ \text { Total } & 170.684 & 46.988 & 103.511\end{array}$

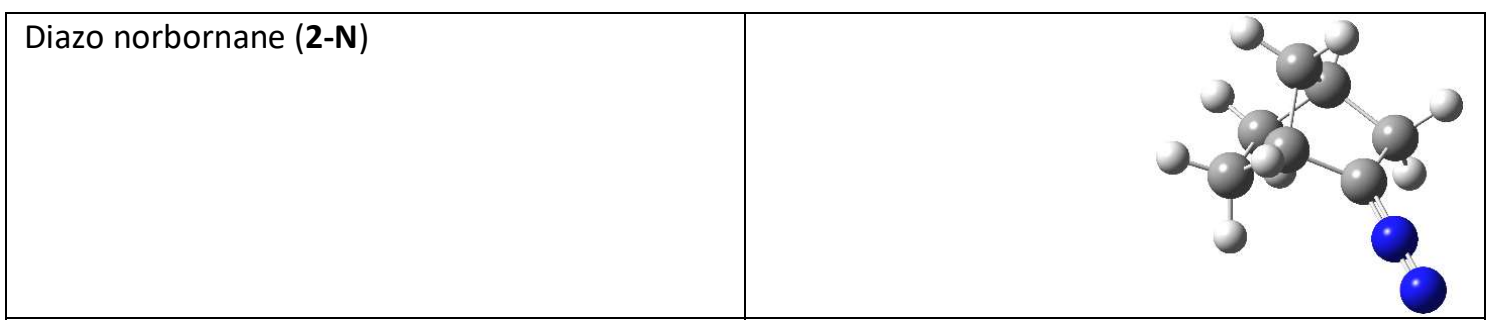

optimized xyz-matrix

C - $1.36245200000 .9455860000-0.0442860000$

C $0.07859600001 .4017480000-0.3614960000$

C 0.83612300000 .17828400000 .1241770000 
C - $0.1478470000-0.79931600000 .7360360000$

C - $0.9496660000-1.4356930000-0.4206720000$

C $-1.7356570000-0.2162580000-0.9933910000$

$\mathrm{H}-1.46376900000 .0067860000-2.0296180000$

H - $2.8140800000-0.3985860000-0.9700730000$

H - $1.6329560000-2.2018690000-0.0379970000$

H - $0.2957430000-1.9053030000-1.1594330000$

C - 1.17800400000 .20413800001 .2932200000

H - $2.0955960000-0.28365300001 .6386970000$

H - 0.77390100000 .83717600002 .0886850000

H $0.2895980000-1.50620300001 .4431580000$

H 0.34261300002 .30576100000 .2025300000

H $0.22187600001 .6112150000-1.4276840000$

H - $2.08241500001 .7667260000-0.0431390000$

N 2.0861370000 -0.0420990000 -0.0504180000

N $3.2078380000-0.2297570000-0.1933900000$

\section{Thermodynamic Data}

Zero-point correction $=0.165485$ (Hartree/Particle)

Thermal correction to Energy $=0.172687$

Thermal correction to Enthalpy $=0.173631$

Thermal correction to Gibbs Free Energy= 0.133389

Sum of electronic and zero-point Energies $=-381.863098$

Sum of electronic and thermal Energies $=-381.855897$

Sum of electronic and thermal Enthalpies $=-381.854953$

Sum of electronic and thermal Free Energies $=-\mathbf{3 8 1 . 8 9 5 1 9 5}$

\begin{tabular}{llrr} 
& E (Thermal) & CV & \multicolumn{1}{c}{ S } \\
KCal/Mol & Cal/Mol-Kelvin & Cal/Mol-Kelvin \\
Total & 108.363 & 28.481 & 84.696
\end{tabular}

\begin{tabular}{|l|l|}
\hline Diazo bornane (2-B) & 0 \\
\hline
\end{tabular}

optimized xyz-matrix

C - 0.97007800001 .21434600000 .5781620000

C 0.48644700001 .37485600001 .0662410000

C 1.13108100000 .27553700000 .2443210000

C $0.0639800000-0.4610270000-0.5567310000$

C $-0.35394200000 .5197300000-1.6787760000$

C - $1.01718600001 .6885780000-0.8930730000$

H - $0.47787100002 .6318170000-1.0201390000$

H - $2.04515800001 .8613910000-1.2230330000$

$\mathrm{H}-1.05786800000 .0439630000-2.3713330000$

H $0.51442100000 .8377390000-2.2621200000$

C - $1.1299260000-0.32525200000 .4462490000$

C - $2.4844890000-0.7491680000-0.1274860000$

C - $0.9153090000-1.09240600001 .7523460000$ 
C $0.4464380000-1.8531350000-1.0146000000$ H 0.57921000001 .20457200002 .1460180000 H 0.88149400002 .37337800000 .8481890000 $\mathrm{H}-1.70143800001 .70142700001 .2302060000$ N 2.3869840000 0.03797400000 .1592480000 N $3.5079280000-0.19120800000 .0807920000$ H $1.3118880000-1.8202260000-1.6860320000$ H - $0.3792140000-2.3203220000-1.5614280000$ H $0.7064610000-2.4915540000-0.1635430000$ H -1.0862480000 -2.1632900000 1.5955970000 H -1.6272870000 -0.7546560000 2.5140640000 H $0.0959870000-0.97550800002 .1500540000$ H -3.2704080000 - 0.57561100000 .6162210000 H - $2.4846860000-1.8206840000-0.3573970000$ H - $2.7657800000-0.2121500000-1.0355130000$

\section{Thermodynamic Data}

Zero-point correction $=0.250364$ (Hartree/Particle) Thermal correction to Energy $=0.261764$ Thermal correction to Enthalpy $=0.262708$ Thermal correction to Gibbs Free Energy= 0.214231 Sum of electronic and zero-point Energies $=-499.665323$ Sum of electronic and thermal Energies $=-499.653922$ Sum of electronic and thermal Enthalpies $=-499.652978$ Sum of electronic and thermal Free Energies $=-499.701455$

\begin{tabular}{llrr} 
& E (Thermal) & CV & \multicolumn{1}{c}{ S } \\
& KCal/Mol & Cal/Mol-Kelvin & Cal/Mol-Kelvin \\
Total & 164.259 & 45.946 & 102.029
\end{tabular}

diazo fenchane (2-F)

optimized xyz-matrix

C - $0.1878820000-1.59086700000 .3768060000$

C - $1.1695580000-0.39935400000 .1526280000$

C - 0.12932100000 .70757000000 .0066950000

C 1.27156700000 .14436600000 .1783270000

C $1.5622970000-0.7203130000-1.0770240000$

C $0.5878060000-1.9235560000-0.9160890000$

$\mathrm{H}-0.0600860000-2.0746500000-1.7819680000$

H $1.1460760000-2.8534380000-0.7698290000$

H $2.6076650000-1.0515730000-1.0729350000$

H $1.3960610000-0.1519610000-1.9969610000$

C $0.9425910000-0.95532300001 .2105950000$

H $1.7826930000-1.64188400001 .3683780000$

H $0.6264730000-0.54680200002 .1742980000$

C 2.34173700001 .15574000000 .5324390000 
C - $2.0714310000-0.19190600001 .3781210000$

C $-2.0608120000-0.5393760000-1.0859660000$

H - $0.6911290000-2.45464100000 .8220590000$

N $-0.40050500001 .9216940000-0.2986270000$

$\mathrm{N}-0.65218200003 .0102230000-0.5594980000$

H 2.08155500001 .69768800001 .4473120000

H $2.47086200001 .8904780000-0.2704150000$

H 3.30324600000 .65579600000 .6867150000

H - $2.76394900000 .2993490000-1.1381360000$

H - 2.6473930000 -1.4645000000 -1.0406410000

H - $1.4807980000-0.5380800000-2.0120420000$

H - 2.8005850000 -1.0066980000 1.4568190000

H -2.6255160000 0.74869000001 .2869550000

H - $1.4983280000-0.15308200002 .3080640000$

\section{Thermodynamic Data}

Zero-point correction $=0.249714$ (Hartree/Particle)

Thermal correction to Energy $=0.261324$

Thermal correction to Enthalpy $=0.262268$

Thermal correction to Gibbs Free Energy $=0.213025$

Sum of electronic and zero-point Energies $=-499.666384$

Sum of electronic and thermal Energies $=-499.654773$

Sum of electronic and thermal Enthalpies $=-499.653829$

Sum of electronic and thermal Free Energies $=-499.703072$

\begin{tabular}{llrr} 
& E (Thermal) & CV & \multicolumn{1}{c}{ S } \\
KCal/Mol & Cal/Mol-Kelvin & Cal/Mol-Kelvin \\
Total & 163.983 & 46.201 & 103.642
\end{tabular}

\begin{tabular}{|l|l|}
\hline Diazo norbornane endo dissociation (endoTS-2- \\
N)
\end{tabular}

optimized xyz-matrix

C $1.4537950000-0.5501730000-0.6543630000$

C $0.1853970000-1.4002690000-0.4991810000$

C $-0.5691070000-0.80560500000 .6971020000$

C 0.35182600000 .32682500001 .1308890000

C 0.39371200001 .48917300000 .1070980000

C $1.03250400000 .8464730000-1.1539640000$

H $0.32557600000 .7739900000-1.9871100000$

H $1.89163000001 .4267630000-1.5019380000$

H 1.03950900002 .27801400000 .5088100000

H $-0.58838600001 .9297870000-0.0743890000$

C $1.7433380000-0.30008700000 .8359080000$

H 2.54970400000 .41596600001 .0266700000

H $1.9433160000-1.22111500001 .3925990000$

H 0.18253400000 .66543000002 .1559490000 
H $0.4506700000-2.4239790000-0.1965840000$

H - $0.4343120000-1.4953830000-1.3961880000$

H 2.2554280000 -1.0173280000 -1.2310040000

$\mathrm{N}-2.10232400000 .0440500000-0.1130390000$

$\mathrm{N}-3.20688400000 .1030690000-0.0980690000$

\section{Thermodynamic Data}

Zero-point correction $=0.161089$ (Hartree/Particle)

Thermal correction to Energy $=0.168685$

Thermal correction to Enthalpy $=0.169629$

Thermal correction to Gibbs Free Energy $=0.128510$

Sum of electronic and zero-point Energies $=-381.812149$

Sum of electronic and thermal Energies $=-381.804553$

Sum of electronic and thermal Enthalpies $=-381.803609$

Sum of electronic and thermal Free Energies $=-381.844728$

\begin{tabular}{llrr} 
& E (Thermal) & CV & \multicolumn{1}{c}{ S } \\
KCal/Mol & Cal/Mol-Kelvin & Cal/Mol-Kelvin \\
Total & 105.852 & 29.297 & 86.543
\end{tabular}

\begin{tabular}{|l|l|l|}
\hline Diazo norbornane exo dissociation (exoTS-2-N) &
\end{tabular}

optimized xyz-matrix C -1.1677100000 1.01201500000 .4130600000

C - $0.01857300001 .2180730000-0.5999670000$

C $0.6475320000-0.1621450000-0.7686840000$

C $-0.1204440000-0.98466300000 .2384850000$

C - $1.4658780000-1.1752390000-0.5358890000$

C - $2.22820800000 .1578880000-0.2982890000$

H - $2.57553700000 .6151650000-1.2295500000$

H -3.1005300000 0.00315800000 .3444300000

H - $1.9994340000-2.0340260000-0.1207970000$

H - $1.2892040000-1.3794280000-1.5964870000$

C - $0.5453180000-0.01977900001 .3636450000$

H -1.2728100000 -0.4506370000 2.0608870000

H 0.31187900000 .36480100001 .9274130000

H $0.3473680000-1.92822200000 .5267900000$

H $0.71113500001 .9517470000-0.2437510000$

H - $0.36711500001 .5765480000-1.5759670000$

H - 1.54813000001 .92913400000 .8682910000

N 2.3234180000 0.01008900000 .0570410000

N $3.4157200000-0.1422500000-0.0335310000$

\section{Thermodynamic Data}

Zero-point correction $=0.160972$ (Hartree/Particle)

Thermal correction to Energy $=0.168590$

Thermal correction to Enthalpy $=0.169534$

Thermal correction to Gibbs Free Energy= 0.128598

Sum of electronic and zero-point Energies $=-\mathbf{3 8 1 . 8 1 7 5 0 6}$ 
Sum of electronic and thermal Energies $=-381.809888$

Sum of electronic and thermal Enthalpies $=-381.808944$

Sum of electronic and thermal Free Energies $=-381.849880$

\begin{tabular}{rlrr} 
& E (Thermal) & CV & \multicolumn{1}{c}{ S } \\
KCal/Mol & Cal/Mol-Kelvin & Cal/Mol-Kelvin \\
Total & 105.792 & 29.411 & 86.157
\end{tabular}

\begin{tabular}{|l|r|c|}
\hline Diazo bornane endo dissociation (endoTS-2-B) & & \\
\hline
\end{tabular}

optimized xyz-matrix

C $0.8302840000-1.40373400000 .2580890000$

C - $0.4153390000-1.25471600001 .1433660000$

C - 0.91212000000 .17536900000 .8808740000

C $-0.01389000000 .6541350000-0.2558420000$

C $-0.2192310000-0.1877970000-1.5439770000$

C $0.3281680000-1.5944340000-1.1849340000$

H $-0.4444950000-2.3677220000-1.2351580000$

H $1.1308470000-1.8984330000-1.8626340000$

H $0.35126800000 .2671760000-2.3614390000$

H - $1.2655980000-0.1960220000-1.8571470000$

C 1.35493400000 .05812300000 .2596500000

C $2.53326900000 .3027620000-0.6841900000$

C 1.76332500000 .58267000001 .6442360000

C $-0.07528500002 .1540130000-0.4860960000$

H - $0.1771430000-1.33457300002 .2109680000$

H -1.1867990000 -2.0068420000 0.9508520000

H $1.5487410000-2.15873200000 .5930420000$

N -2.6802990000 -0.02939000000 .0871480000$

$\mathrm{N}-3.73432000000 .29656000000 .1646050000$

H - $1.06935800002 .4419410000-0.8462990000$

H $0.65760200002 .4782040000-1.2336730000$

H 0.10530200002 .69839900000 .4458480000

H 0.95012200000 .57045900002 .3745650000

H 2.1071770000 1.61978400001 .5637600000

H $2.5946550000-0.00997700002 .0431380000$

H $2.75029800001 .3738160000-0.7619030000$

H $2.3762280000-0.0827990000-1.6934700000$

H $3.4287970000-0.1832120000-0.2797850000$

\section{Thermodynamic Data}

Zero-point correction $=0.245496$ (Hartree/Particle)

Thermal correction to Energy $=0.257492$

Thermal correction to Enthalpy $=0.258436$

Thermal correction to Gibbs Free Energy $=0.208446$

Sum of electronic and zero-point Energies $=-499.611808$ 
Sum of electronic and thermal Energies $=-499.599812$

Sum of electronic and thermal Enthalpies $=-499.598868$

Sum of electronic and thermal Free Energies $=-499.648858$ $\mathrm{E}$ (Thermal)

CV $\mathrm{S}$

Total Cal/Mol-Kelvin Cal/Mol-Kelvin 47.063 105.214

\begin{tabular}{|l|l|l|l|}
\hline exo-diazo bornane dissociation TS (exoTS-2-B) &
\end{tabular}

optimized xyz-matrix

C - $1.1309840000-0.7536020000-1.0139320000$

C $0.2076370000-1.5233320000-0.9277250000$

C $0.8908880000-1.01456600000 .3536950000$

C -0.02546700000 .10884100000 .7715340000$

C - $1.1606530000-0.80093200001 .3769960000$

C - $1.9768470000-1.28856100000 .1519590000$

H - $2.0704090000-2.37786600000 .1202020000$

H - $2.9880380000-0.87285600000 .1465350000$

H - $1.7567170000-0.19953700002 .0697860000$

H - $0.7303180000-1.62516300001 .9550260000$

C - $0.72786700000 .6548610000-0.5186610000$

C - $1.90476000001 .5897250000-0.2153880000$

C $0.19468000001 .3987840000-1.4882780000$

C 0.53233000001 .11437700001 .7582250000

H $0.8459380000-1.3535890000-1.7992020000$

H $0.0637530000-2.6086660000-0.8656550000$

H -1.6197390000 - $0.7891260000-1.9926150000$

N 2.4662280000 -0.1560690000 -0.1871200000

N $3.5583660000-0.0667510000-0.0257250000$

H $0.66244700002 .2604180000-0.9973330000$

H - $0.39122800001 .7822470000-2.3311530000$

H $0.99166300000 .7785560000-1.9018430000$

H - 2.5609270000 1.22739400000 .5796320000

H - 2.5164820000 $1.7369340000-1.1129380000$

H - 1.53243100002 .57399100000 .0909540000

H - 0.25622100001 .77335900002 .1381720000

H 1.30122000001 .74185300001 .2928570000

H 0.99158100000 .59823000002 .6069370000

\section{Thermodynamic Data}

Zero-point correction $=0.245578$ (Hartree/Particle)

Thermal correction to Energy $=0.257407$

Thermal correction to Enthalpy $=0.258352$

Thermal correction to Gibbs Free Energy= 0.209086

Sum of electronic and zero-point Energies $=-499.617929$

Sum of electronic and thermal Energies $=-499.606100$

Sum of electronic and thermal Enthalpies $=-499.605156$ 
Sum of electronic and thermal Free Energies $=-499.654421$

\begin{tabular}{llrr} 
& E (Thermal) & CV & \multicolumn{1}{c}{ S } \\
KCal/Mol & Cal/Mol-Kelvin & Cal/Mol-Kelvin \\
Total & 161.526 & 46.960 & 103.688
\end{tabular}

\begin{tabular}{|l|l|l|}
\hline endo diazo fenchane dissociation (endo-TS-2-F) & \\
\hline
\end{tabular}

optimized xyz-matrix

C $1.4041820000-0.93328200000 .2547260000$

C $1.13501400000 .5210370000-0.2212540000$

C - $0.30993300000 .4437840000-0.7651550000$

C $-0.8088540000-0.9167810000-0.3233600000$

C - $0.7924470000-1.11339200001 .2201520000$

C $0.7092580000-1.20026400001 .5965040000$

H $1.0025830000-0.50075700002 .3825190000$

H $0.9617880000-2.20467700001 .9508090000$

H -1.3146410000 -2.0461330000 1.4639220000

H - $1.3215480000-0.30426800001 .7315450000$

C $0.4801730000-1.7069070000-0.7041530000$

H $0.3680520000-2.7694430000-0.4593740000$

H $0.7469860000-1.6196920000-1.7617580000$

C - $2.1064490000-1.3497350000-0.9826410000$

C $2.03944400000 .8604770000-1.4224500000$

C 1.35904600001 .60785100000 .8343550000

H 2.4633520000 -1.2095360000 0.2386610000

N - 1.42619400001 .69015000000 .2543290000

$\mathrm{N}-2.18982100002 .48326700000 .1548000000$

H $-2.3307350000-2.4004490000-0.7667850000$

H - $2.0503340000-1.2149120000-2.0663600000$

H - $2.9441700000-0.7456260000-0.6150430000$

H $1.75234400001 .8287900000-1.8416730000$

H $1.96180100000 .1189660000-2.2221530000$

H $3.08703200000 .9054330000-1.1018130000$

H 1.15789400002 .59515200000 .4053830000

H 2.40220400001 .59163400001 .1703850000

H 0.71288700001 .48486400001 .7074750000

\section{Thermodynamic Data}

Zero-point correction $=0.245865$ (Hartree/Particle)

Thermal correction to Energy $=0.257704$

Thermal correction to Enthalpy $=0.258648$

Thermal correction to Gibbs Free Energy= 0.209115

Sum of electronic and zero-point Energies $=-499.612478$

Sum of electronic and thermal Energies $=-499.600639$

Sum of electronic and thermal Enthalpies $=-499.599694$

Sum of electronic and thermal Free Energies $=-499.649227$ 


\begin{tabular}{llrr} 
& E (Thermal) & CV & \multicolumn{1}{c}{ S } \\
& KCal/Mol & Cal/Mol-Kelvin & Cal/Mol-Kelvin \\
Total & 161.712 & 46.872 & 104.251
\end{tabular}

\begin{tabular}{|l|l|l|l|}
\hline exo-diazo fenchane dissociation TS (exo-TS-2-F) &
\end{tabular}

\section{optimized xyz-matrix}

C $1.3318020000-0.33300600000 .8871470000$

C $0.3758150000-1.1447740000-0.0441080000$

C $-0.5011370000-0.0614910000-0.7175310000$

C - $0.04720000001 .2131700000-0.0398230000$

C $1.33749200001 .4042640000-0.7564440000$

C 2.30557400000 .47593400000 .0170650000

H $2.9289820000-0.1403510000-0.6348820000$

H 2.97666200001 .06039700000 .6542630000

H $1.62301300002 .4583720000-0.6838320000$

H $1.26685800001 .1594100000-1.8225120000$

C 0.40603100000 .78863300001 .3748180000

H 0.94028300001 .57878400001 .9162930000

H - 0.43585000000 .44863600001 .9879540000

C - $0.96116100002 .4160640000-0.1652100000$

C - $0.4195410000-2.16538500000 .7807800000$

C $1.0990270000-1.9217500000-1.1562670000$

H $1.8123060000-0.94532700001 .6569260000$

N - $2.2532020000-0.2576790000-0.0337200000$

$\mathrm{N}-3.3286540000-0.3348090000-0.2818620000$

$\mathrm{H}-1.89000700002 .26841300000 .3966720000$

H - $1.22352200002 .5910210000-1.2133330000$

H -0.47437800003 .31572500000 .2267630000$

H $-0.8825530000-1.71517500001 .6635210000$

H $0.2528610000-2.96230100001 .1173860000$

H - $1.2075950000-2.62353300000 .1741450000$

H $0.3884420000-2.5700140000-1.6771410000$

H $1.8945780000-2.5446820000-0.7294070000$

H $1.5426930000-1.2619080000-1.9063180000$

\section{Thermodynamic Data}

Zero-point correction $=0.246093$ (Hartree/Particle) Thermal correction to Energy $=0.257742$

Thermal correction to Enthalpy $=0.258686$

Thermal correction to Gibbs Free Energy $=0.209851$

Sum of electronic and zero-point Energies $=-499.618245$

Sum of electronic and thermal Energies $=-499.606596$

Sum of electronic and thermal Enthalpies $=-499.605652$

Sum of electronic and thermal Free Energies $=-499.654487$

$$
\mathrm{E} \text { (Thermal) }
$$

CV 


$\begin{array}{llrr} & \mathrm{KCal} / \mathrm{Mol} & \mathrm{Cal} / \mathrm{Mol}-\text { Kelvin } & \text { Cal/Mol-Kelvin } \\ \text { Total } & 161.735 & 46.592 & 102.782\end{array}$

4.2.3 Structures optimized at the B3LYP/6-31G* level of theory

Diazo norbornane (2-N)

optimized xyz-matrix

C - $1.36493700000 .9528440000-0.0347440000$

C $0.08249600001 .3999400000-0.3691070000$

C 0.84548600000 .17311900000 .1169290000

C $-0.1466130000-0.80541800000 .7294720000$

C $-0.9679090000-1.4358440000-0.4279610000$

C $-1.7656840000-0.2083570000-0.9840440000$

$\mathrm{H}-1.51768600000 .0151690000-2.0273500000$

H $-2.8456310000-0.3863750000-0.9391710000$

$\mathrm{H}-1.6447200000-2.2083510000-0.0420340000$

H - $0.3258290000-1.9037490000-1.1799230000$

C - 1.16966700000 .20476600001 .3042240000

H - $2.0860670000-0.27789000001 .6633140000$

H - 0.75609000000 .83237200002 .1007010000

H $0.2910790000-1.52245800001 .4280020000$

H 0.35684500002 .31019500000 .1837770000

H $0.21826300001 .6080210000-1.4383350000$

H - $2.07687000001 .7827230000-0.0235680000$

N $2.0988580000-0.0455200000-0.0543280000$

N $3.2308100000-0.2310470000-0.1933900000$

\section{Thermodynamic Data}

Zero-point correction $=0.163652$ (Hartree/Particle)

Thermal correction to Energy $=0.170926$

Thermal correction to Enthalpy $=0.17187040$

Thermal correction to Gibbs Free Energy= 0.131588

Sum of electronic and zero-point Energies $=-382.035064$

Sum of electronic and thermal Energies $=-382.027790$

Sum of electronic and thermal Enthalpies $=-382.026846$

Sum of electronic and thermal Free Energies $=-\mathbf{3 8 2 . 0 6 7 1 2 8}$

\begin{tabular}{rlcr} 
& E (Thermal) & CV & \multicolumn{1}{c}{ S } \\
KCal/Mol & Cal/Mol-Kelvin & Cal/Mol-Kelvin \\
Total & 107.25828. & 900 & 84.782
\end{tabular}




\begin{tabular}{|l|l|}
\hline Endo-diazo norbornane dissociation TS (endo- \\
TS-2-N)
\end{tabular}

\section{optimized xyz-matrix}

C $1.4971720000-0.5898470000-0.6113170000$

C $0.1738980000-1.3784400000-0.5238740000$

C - $0.5993730000-0.76413900000 .6475140000$

C 0.33241100000 .33522800001 .1200800000

C 0.45618000001 .49262600000 .0788090000

C $1.18116200000 .8319750000-1.1326530000$

H $0.55537200000 .8047000000-2.0314500000$

H $2.09647900001 .3748280000-1.3919180000$

H 1.07050000002 .28772100000 .5189070000

H - $0.51277800001 .9271680000-0.1750100000$

C $1.7212890000-0.34695800000 .8971880000$

H 2.54498000000 .33724100001 .1318210000

H $1.8588820000-1.27180200001 .4684670000$

H 0.12384000000 .69563200002 .1319100000

H $0.3706520000-2.4234120000-0.2335990000$

H - $0.4139670000-1.4303950000-1.4467390000$

H 2.3037100000 -1.1005440000 -1.1456380000

N - $2.19836900000 .0965190000-0.1586070000$

N-3.3122170000 $0.0915080000-0.0815710000$

\section{Thermodynamic Data}

Zero-point correction $=0.158994$ (Hartree/Particle)

Thermal correction to Energy $=0.166793$

Thermal correction to Enthalpy $=0.167737$

Thermal correction to Gibbs Free Energy $=0.126186$

Sum of electronic and zero-point Energies $=-381.982831$

Sum of electronic and thermal Energies $=-\mathbf{3 8 1 . 9 7 5 0 3 2}$

Sum of electronic and thermal Enthalpies $=-381.97408741$

Sum of electronic and thermal Free Energies $=-\mathbf{3 8 2} .015639$

\begin{tabular}{llrr} 
& E (Thermal) & CV & \multicolumn{1}{c}{ S } \\
KCal/Mol & Cal/Mol-Kelvin & Cal/Mol-Kelvin \\
Total & 104.664 & 29.880 & 87.452
\end{tabular}

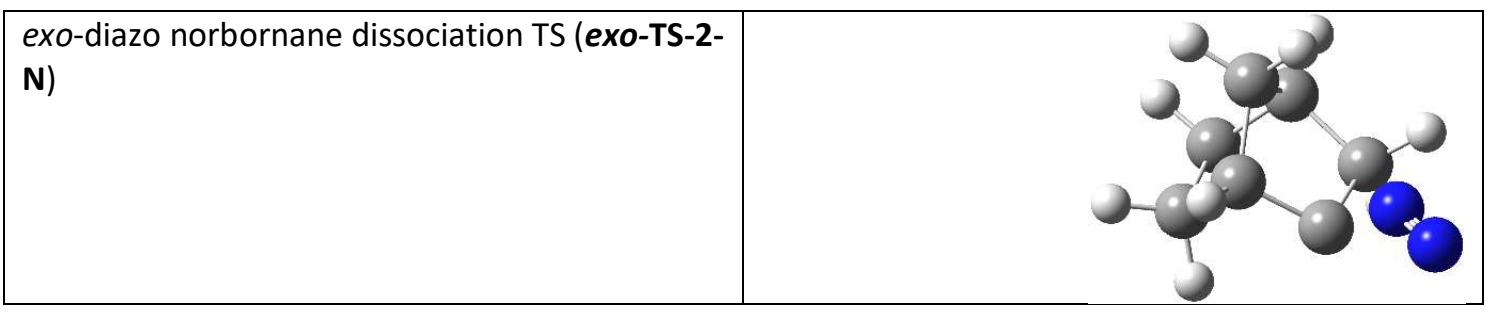

optimized xyz-matrix 
C - 1.22568700001 .01637400000 .3736820000

C $-0.02673700001 .2238550000-0.5897630000$

C $0.6521320000-0.1513240000-0.7110170000$

C - $0.1246670000-0.96669300000 .2889820000$

C $-1.4485980000-1.2086940000-0.5326490000$

C - $2.24238100000 .1226300000-0.3674170000$

H - $2.55242100000 .5490560000-1.3275710000$

H -3.1458820000 -0.0270280000 0.2341540000

H - $1.9761320000-2.0644370000-0.1001990000$

H - $1.2362730000-1.4527400000-1.5789190000$

C - 0.62026500000 .01775900001 .3790610000

H - $1.3638250000-0.41591900002 .0592850000$

H 0.20308700000 .43378400001 .9710450000

H $0.3502440000-1.89487600000 .6155720000$

H $0.68575500001 .9555500000-0.1926680000$

H - $0.31747300001 .5896320000-1.5836740000$

H - 1.64405100001 .93720100000 .7901010000

N 2.40029000000 .02573200000 .0927160000

N $3.4874520000-0.1591130000-0.0830590000$

\section{Thermodynamic Data}

Zero-point correction $=0.159142$ (Hartree/Particle)

Thermal correction to Energy $=0.166904$

Thermal correction to Enthalpy $=0.167848$

Thermal correction to Gibbs Free Energy $=0.126516$

Sum of electronic and zero-point Energies $=-\mathbf{3 8 1 . 9 8 8 0 1 0}$

Sum of electronic and thermal Energies $=-381.980248$

Sum of electronic and thermal Enthalpies $=-381.979304$

Sum of electronic and thermal Free Energies $=-\mathbf{3 8 2 . 0 2 0 6 3 6}$

\begin{tabular}{llrr} 
& E (Thermal) & CV & \multicolumn{1}{c}{ S } \\
KCal/Mol & Cal/Mol-Kelvin & Cal/Mol-Kelvin \\
Total & 104.734 & 29.839 & 86.990
\end{tabular}

\begin{tabular}{|l|l|}
\hline Norbornyl endo-diazonium ion $\left(\right.$ endo- $\left.\mathbf{N}-\mathrm{N}_{2}{ }^{+}\right)$ & \\
\hline
\end{tabular}

optimized xyz-matrix

C $1.4336630000-0.6082370000-0.5717140000$

C $0.0703570000-1.3233260000-0.7170160000$

C $-0.7346430000-0.69609000000 .4876480000$

C 0.23298300000 .36828400001 .1060860000

C 0.45986000001 .52235700000 .1035140000

C $1.29065300000 .8565420000-1.0432940000$

H $0.80551200000 .9341100000-2.0213460000$

H 2.2733740000 1.3264360000 -1.1351050000

H 1.02712500002 .31079200000 .6055080000 
H -0.4676070000 $1.9916120000-0.2437440000$

C $1.5411260000-0.44979100000 .9602820000$

H 2.40520700000 .13888000001 .2819310000

H $1.5398640000-1.39663400001 .5109230000$

H - 0.06842300000 .66379700002 .1123960000

H $0.1346420000-2.4021930000-0.5545380000$

H - $0.4132790000-1.1542300000-1.6832100000$

H 2.2390010000 -1.1651980000 -1.0549260000

$\mathrm{N}-1.9778270000-0.09358000000 .0340890000$

$\mathrm{N}-2.90265300000 .4035760000-0.3184670000$

H -1.0760490000 -1.4357760000 1.2197140000

\section{Thermodynamic Data}

Zero-point correction $=0.175907$ (Hartree/Particle)

Thermal correction to Energy $=0.183422$

Thermal correction to Enthalpy $=0.184366$

Thermal correction to Gibbs Free Energy= 0.143943

Sum of electronic and zero-point Energies $=-382.393977$

Sum of electronic and thermal Energies $=-382.386462$

Sum of electronic and thermal Enthalpies $=-382.385518$

Sum of electronic and thermal Free Energies $=-\mathbf{3 8 2 . 4 2 5 9 4 1}$

\begin{tabular}{llrr} 
& E (Thermal) & CV & \multicolumn{1}{c}{ S } \\
KCal/Mol & Cal/Mol-Kelvin & Cal/Mol-Kelvin \\
Total & 115.099 & 30.291 & 85.077
\end{tabular}

\begin{tabular}{|l|l|}
\hline Norbornyl exo-diazonium ion $\left(\right.$ exo-N-N $\left.{ }^{+}\right)$ &
\end{tabular}

optimized xyz-matrix C -1.1034960000 1.03867700000 .4063090000

C $0.09650600001 .3048390000-0.5375120000$

C $0.7248900000-0.1326100000-0.6672270000$

C $-0.1357150000-1.01608100000 .2950570000$

C - $1.4767820000-1.2011250000-0.4675870000$

C - $2.15739000000 .2010740000-0.3530680000$

H $-2.40820600000 .6307530000-1.3273430000$

H -3.0847730000 0.14104900000 .2227470000

H - $2.0546750000-1.97773800000 .0410640000$

H - $-1.3349720000-1.5368620000-1.4999070000$

C -0.51167900000 .00485600001 .3878840000$

$\mathrm{H}-1.2497950000-0.40290500002 .0841380000$

H 0.33915800000 .37772300001 .9709570000

H $0.3534080000-1.94607000000 .5917300000$

H $0.80768100002 .0083940000-0.0946090000$

H -0.1772720000 $1.6790910000-1.5268120000$

H - 1.48486400001 .96065600000 .8492630000

N $2.1172230000-0.1143210000-0.2022510000$

N $3.1484640000-0.11584000000 .2011030000$

H $0.8165080000-0.5207430000-1.6863310000$ 
Thermodynamic Data

Zero-point correction $=0.175775$ (Hartree/Particle)

Thermal correction to Energy $=0.183328$

Thermal correction to Enthalpy $=0.184272$

Thermal correction to Gibbs Free Energy $=0.143792$

Sum of electronic and zero-point Energies $=-382.394814$

Sum of electronic and thermal Energies $=-382.387261$

Sum of electronic and thermal Enthalpies $=-382.386316$

Sum of electronic and thermal Free Energies $=-\mathbf{3 8 2 . 4 2 6 7 9 7}$

\begin{tabular}{rrrr} 
& E (Thermal) & CV & \multicolumn{1}{c}{ S } \\
& KCal/Mol & Cal/Mol-Kelvin & Cal/Mol-Kelvin \\
Total & 115.040 & 30.386 & 85.198
\end{tabular}

\begin{tabular}{|l|l|l|}
\hline endo-Norbornyl diazonium ion dissociation TS \\
(endo-N-N ${ }^{+}$-TS)
\end{tabular}

\section{optimized xyz-matrix}

C $1.4880970000-0.5122530000-0.6558800000$

C $0.1544230000-1.2975320000-0.7421980000$

C $-0.5501500000-0.79678900000 .5227110000$

C 0.28561300000 .27939800001 .1404260000

C 0.42039500001 .50318500000 .1939510000

C $1.23374300000 .9645300000-1.0260870000$

H $0.69642800001 .0691440000-1.9735220000$

H 2.1796420000 $1.5021350000-1.1320530000$

H 0.96057300002 .28971500000 .7269110000

H $-0.55300900001 .9221070000-0.0814080000$

C $1.6634090000-0.44464600000 .8786260000$

H $0.2981060000-2.3793120000-0.6398220000$

H - $0.4107760000-1.1337890000-1.6644730000$

H $2.2947630000-0.9903370000-1.2134290000$

N $-2.1365450000-0.06933700000 .0070570000$

$\mathrm{N}-3.13085100000 .3237300000-0.2675940000$

H - $1.0446500000-1.52021000001 .1715980000$

H 2.47972200000 .19835500001 .2209180000

H $1.7629700000-1.42120700001 .3635040000$

H 0.03482500000 .50728400002 .1762390000

\section{Thermodynamic Data}

Zero-point correction $=0.173778$ (Hartree/Particle)

Thermal correction to Energy $=0.181559$

Thermal correction to Enthalpy $=0.182503$

Thermal correction to Gibbs Free Energy= 0.141108

Sum of electronic and zero-point Energies $=-\mathbf{3 8 2 . 3 9 0 5 9 5}$

Sum of electronic and thermal Energies $=-382.382815$

Sum of electronic and thermal Enthalpies $=-\mathbf{3 8 2 . 3 8 1 8 7 1}$ 
Sum of electronic and thermal Free Energies $=-382.423266$

\begin{tabular}{llrr} 
& E (Thermal) & CV & \multicolumn{1}{c}{ S } \\
KCal/Mol & Cal/Mol-Kelvin & Cal/Mol-Kelvin \\
Total & 113.930 & 30.007 & 87.123
\end{tabular}

\begin{tabular}{|l|l|}
\hline $\begin{array}{l}\text { exo-Norbornyl diazonium ion dissociation TS } \\
\text { (exo-N-N } \mathbf{N}_{2}{ }^{+} \text {-TS) }\end{array}$ & \\
\hline
\end{tabular}

optimized xyz-matrix

C - 1.11226300001 .03634800000 .4288450000

C $0.02926500001 .2566010000-0.6076540000$

C $0.6120760000-0.1583850000-0.7287840000$

C $-0.1368970000-1.01445700000 .2626110000$

C $-1.5288010000-1.1738320000-0.4623520000$

C - $2.21977800000 .2079440000-0.2541370000$

H - 2.5601200000 0.6544580000 -1.1927600000

H -3.0907870000 0.11687900000 .4003060000

H - $2.0595430000-1.98779000000 .0393270000$

H - $-1.4322470000-1.4625550000-1.5142190000$

C - $0.4744150000-0.00099700001 .3785930000$

H $0.76650000001 .9743820000-0.2354220000$

H - $0.30253300001 .6130740000-1.5876410000$

H - 1.44572000001 .96998900000 .8843360000

N 2.2521380000 -0.0876470000 -0.1860600000

N $3.2771930000-0.08737900000 .2245220000$

H $0.8460720000-0.5664980000-1.7120950000$

H -1.1791140000 -0.4104870000 2.1069190000

H 0.40432900000 .36926100001 .9201050000

H $0.3327200000-1.96485900000 .5191810000$

Thermodynamic Data

Zero-point correction $=0.173923$ (Hartree/Particle)

Thermal correction to Energy $=0.181602$

Thermal correction to Enthalpy $=0.182546$

Thermal correction to Gibbs Free Energy $=0.141462$

Sum of electronic and zero-point Energies $=-382.393900$

Sum of electronic and thermal Energies $=-382.386221$

Sum of electronic and thermal Enthalpies $=-382.385277$

Sum of electronic and thermal Free Energies $=-\mathbf{3 8 2 . 4 2 6 3 6 2}$

\begin{tabular}{llrr} 
& E (Thermal) & CV & \multicolumn{1}{c}{ S } \\
KCal/Mol & Cal/Mol-Kelvin & Cal/Mol-Kelvin \\
Total & 113.957 & 29.817 & 86.470
\end{tabular}




\begin{tabular}{|l|l|l|}
\hline Diazo bornane (2-B) & \\
\hline
\end{tabular}

optimized xyz-matrix

C $0.95869900001 .2287860000-0.5726040000$

C - $0.50605700001 .3733870000-1.0590530000$

C - $1.14324300000 .2485150000-0.2562220000$

C $-0.0632280000-0.47299300000 .5578890000$

C 0.33398600000 .52777100001 .6853000000

C 1.00340500001 .70238400000 .9039640000

H 0.46725400002 .64839600001 .0338390000

H 2.03143900001 .87350800001 .2378840000

H 1.02924700000 .06390700002 .3955650000

H - 0.54429600000 .84388700002 .2564200000

C $1.1449110000-0.3180040000-0.4487150000$

C $2.5127310000-0.72994800000 .1253680000$

C $0.9533060000-1.0839520000-1.7687230000$

C - $0.4253490000-1.87140100001 .0354350000$

H $-0.59900200001 .2273260000-2.1436300000$

H - $0.91691200002 .3642580000-0.8268080000$

H $1.67893300001 .7333080000-1.2247990000$

N -2.4033690000 $0.0195140000-0.1695700000$

N -3.5359480000 -0.2010430000 -0.0997960000

H - $1.2956530000-1.84847900001 .7024680000$

H $0.4038140000-2.31749100001 .5959980000$

H - $0.6679640000-2.53419200000 .1973340000$

H $1.1090440000-2.1583940000-1.6153600000$

H $1.6868440000-0.7534240000-2.5144770000$

H $-0.0453370000-0.9579410000-2.1949020000$

H $3.3041340000-0.5034430000-0.5998390000$

H $2.5449280000-1.81023600000 .3104860000$

H $2.7737880000-0.22755700001 .0595470000$

\section{Thermodynamic Data}

Zero-point correction $=0.247669$ (Hartree/Particle)

Thermal correction to Energy $=0.259276$

Thermal correction to Enthalpy $=0.260221$

Thermal correction to Gibbs Free Energy $=0.211400$

Sum of electronic and zero-point Energies $=-499.891304$

Sum of electronic and thermal Energies $=-499.879697$

Sum of electronic and thermal Enthalpies $=-499.878752$

Sum of electronic and thermal Free Energies $=-499.927572$

\begin{tabular}{llrr} 
& E (Thermal) & CV & \multicolumn{1}{c}{ S } \\
KCal/Mol & Cal/Mol-Kelvin & Cal/Mol-Kelvin \\
Total & 162.698 & 46.648 & 102.751
\end{tabular}




\begin{tabular}{|l|l|}
\hline $\begin{array}{l}\text { endo-diazo bornane dissociation TS } \\
\text { (endo-TS-2-B) }\end{array}$ & \\
\hline
\end{tabular}

\section{optimized xyz-matrix}

C $0.8562360000-1.39662500000 .2941790000$

C - $0.4318920000-1.24909700001 .1297850000$

C -0.93986500000 .16071300000 .8144610000$

C $-0.00988600000 .6488250000-0.2857770000$

C - $0.1693120000-0.2374080000-1.5676620000$

C $0.4117200000-1.6245130000-1.1677260000$

H - $0.3315690000-2.4258770000-1.2378910000$

H $1.2494050000-1.9121800000-1.8108360000$

H $0.39820300000 .2155330000-2.3887700000$

H - $1.2119470000-0.2825920000-1.8899570000$

C 1.36892300000 .07930700000 .2882420000

C $2.57942400000 .3322050000-0.6257190000$

C 1.73735300000 .62925100001 .6823060000

C $-0.10108300002 .1475210000-0.5596970000$

H - $0.2421150000-1.30990600002 .2100810000$

$\mathrm{H}-1.1856430000-2.01659300000 .9215790000$

H $1.5669500000-2.14021700000 .6711580000$

N - $2.7560260000-0.04596200000 .0415010000$

$\mathrm{N}-3.80332800000 .30059400000 .2199150000$

H - $1.08580100002 .4026690000-0.9691010000$

H $0.65350800002 .4752900000-1.2848420000$

H 0.02625000002 .72657000000 .3609050000

H 0.91885500000 .58416900002 .4056270000

H 2.0398940000 1.68028300001 .6079870000

H 2.5883340000 0.07447100002 .0970610000

H $2.80936300001 .4027510000-0.6789600000$

H $2.4487150000-0.0297120000-1.6479830000$

H $3.4633730000-0.1681490000-0.2103250000$

\section{Thermodynamic Data}

Zero-point correction $=0.242764$ (Hartree/Particle)

Thermal correction to Energy $=0.254998$

Thermal correction to Enthalpy $=0.255942$

Thermal correction to Gibbs Free Energy $=0.205520$

Sum of electronic and zero-point Energies $=-499.837183$

Sum of electronic and thermal Energies $=-499.824949$

Sum of electronic and thermal Enthalpies $=-499.824005$

Sum of electronic and thermal Free Energies $=-499.874426$

\begin{tabular}{llrr} 
& E (Thermal) & CV & \multicolumn{1}{c}{ S } \\
& KCal/Mol & Cal/Mol-Kelvin & Cal/Mol-Kelvin \\
Total & 160.013 & 47.805 & 106.121
\end{tabular}




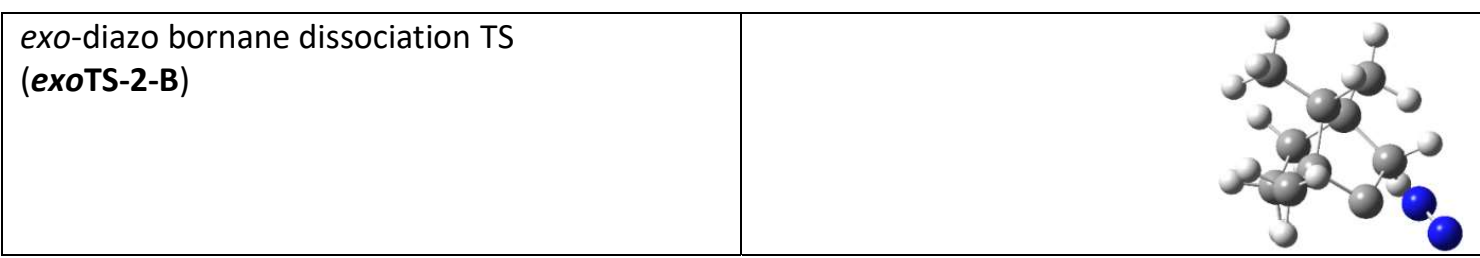

optimized xyz-matrix

C - $1.1254940000-0.7840140000-1.0145670000$

C $0.2765440000-1.4478470000-0.9916200000$

C $0.9420800000-0.93166900000 .2920080000$

C - 0.02264200000 .12237200000 .7672040000

C - $1.0819120000-0.89374700001 .3840320000$

C - $1.8890600000-1.42157100000 .1651330000$

H - $1.8869780000-2.51513000000 .1063530000$

H - 2.9351030000 - 1.10286500000 .1997720000

H - $1.7012750000-0.34284500002 .0989070000$

H - $0.5835310000-1.69215400001 .9440310000$

C - $0.81618300000 .6509490000-0.4973840000$

C - $2.06404600001 .4880250000-0.1519350000$

C $0.02666100001 .4904260000-1.4719000000$

C 0.49929000001 .13424400001 .7763770000

H $0.8764300000-1.1791120000-1.8663660000$

H $0.2313680000-2.5450010000-0.9848120000$

H - $1.6420850000-0.8405120000-1.9785590000$

N $2.5708820000-0.0501100000-0.2207660000$

N $3.6732830000-0.0546520000-0.0328310000$

H $0.31313500002 .4451410000-1.0146190000$

H - $0.55876600001 .7240350000-2.3698010000$

H $0.94525900000 .9967230000-1.7947270000$

$\mathrm{H}-2.71260400001 .02898000000 .5982210000$

H - 2.6696380000 $1.6579980000-1.0509000000$

$\mathrm{H}-1.77110800002 .47328000000 .2299300000$

H - 0.31481200001 .73626300002 .1966580000

H 1.21985500001 .81983500001 .3153990000

H 1.00927300000 .62569000002 .6016040000

\section{Thermodynamic Data}

Zero-point correction $=0.243119$ (Hartree/Particle)

Thermal correction to Energy $=0.255179$

Thermal correction to Enthalpy $=0.256123$

Thermal correction to Gibbs Free Energy= 0.206379

Sum of electronic and zero-point Energies $=-499.842376$

Sum of electronic and thermal Energies $=-499.830316$

Sum of electronic and thermal Enthalpies $=-499.829371$

Sum of electronic and thermal Free Energies $=-499.879115$

\begin{tabular}{llrr} 
& E (Thermal) & CV & \multicolumn{1}{c}{ S } \\
KCal/Mol & Cal/Mol-Kelvin & Cal/Mol-Kelvin \\
Total & 160.127 & 47.633 & 104.695
\end{tabular}




\begin{tabular}{|l|l|}
\hline $\begin{array}{l}\text { Endo-bornane diazonium ion } \\
\left(\text { endo-B- } \mathbf{N}_{2}{ }^{+}\right)\end{array}$ & \\
\hline
\end{tabular}

\section{optimized xyz-matrix}

C - 0.74876400001 .41387500000 .2513290000

C 0.51886700001 .31017100001 .1303800000

C $0.9987260000-0.14914100000 .7956160000$

C $0.0184010000-0.6722180000-0.3327010000$

C $0.26047100000 .1986190000-1.5944480000$

C - $0.28411900001 .6093510000-1.2092170000$

H $0.47438700002 .3936120000-1.2950490000$

H -1.1138860000 1.9049740000 -1.8547140000

H - $0.2864180000-0.2355550000-2.4347340000$

H $1.31112400000 .2102720000-1.9078640000$

C - $1.3112500000-0.04183900000 .2628150000$

C - $2.5232210000-0.2546480000-0.6654580000$

C - $-1.7183650000-0.56055200001 .6562680000$

C $0.0893190000-2.1772090000-0.5424070000$

H 0.32264300001 .37600100002 .2019900000

H 1.26964400002 .06499000000 .8813930000

H - 1.43498700002 .18263900000 .6157330000

N $2.3721430000-0.16422800000 .3488270000$

N $3.4043500000-0.1802480000-0.0557520000$

H $1.0554630000-2.4878250000-0.9584640000$

H - $0.6769900000-2.4881810000-1.2588190000$

H $-0.0753500000-2.73255900000 .3873970000$

H - 2.0040360000 -1.6165430000 1.6096590000

H - $2.5935840000-0.00583600002 .0098510000$

H $-0.9532060000-0.46207600002 .4336980000$

$\mathrm{H}-3.37128000000 .3279650000-0.2901970000$

$\mathrm{H}-2.8283390000-1.3061340000-0.6660010000$

H - $2.35207200000 .0444890000-1.7007870000$

H $1.0210450000-0.81735300001 .6623070000$

\section{Thermodynamic Data}

Zero-point correction $=0.259798$ (Hartree/Particle)

Thermal correction to Energy $=0.271643$

Thermal correction to Enthalpy $=0.272587$

Thermal correction to Gibbs Free Energy= 0.223656

Sum of electronic and zero-point Energies $=-500.254073$

Sum of electronic and thermal Energies $=-500.242228$

Sum of electronic and thermal Enthalpies $=-500.241283$

Sum of electronic and thermal Free Energies $=-\mathbf{5 0 0 . 2 9 0 2 1 4}$

$E$ (Thermal)

$\mathrm{KCal} / \mathrm{Mol}$

Total 170.459
$S$

Cal/Mol-Kelvin

102.984 


\begin{tabular}{|l|l|}
\hline exo-bornane diazonium ion $\left(\right.$ exo-B- $\left.\mathbf{N}_{2}{ }^{+}\right)$ & \\
\hline
\end{tabular}

\section{optimized xyz-matrix}

C - 1.05185800000 .78964300001 .0196210000

C 0.33234500001 .48410000001 .0339890000

C $0.98383000000 .9037750000-0.2697320000$

C - $0.0545770000-0.1652230000-0.8072910000$

C - $1.14885200000 .7785130000-1.4040270000$

C - $1.85223900001 .3947660000-0.1562350000$

H - $1.82003400002 .4883530000-0.1527710000$

H - $2.90528300001 .1093790000-0.1091140000$

H - $1.83248200000 .1784150000-2.0097820000$

H $-0.72341000001 .5292460000-2.0790050000$

C $-0.7262330000-0.65575900000 .5306120000$

C - $1.9734620000-1.52776300000 .2823200000$

C $0.1898220000-1.45674300001 .4758470000$

C $0.5038810000-1.1887690000-1.7844010000$

H 0.91897700001 .23668000001 .9210660000

H 0.27777800002 .57288300000 .9649800000

H - 1.54911700000 .85653700001 .9905410000

N 2.2863530000 0.30773300000 .0045050000

N $3.2730820000-0.16941400000 .1665140000$

H $0.9347270000-0.7120990000-2.6725830000$

H - $-0.3050410000-1.8397020000-2.1286270000$

H $1.2668530000-1.8346560000-1.3346080000$

H $0.5257720000-2.38667200001 .0040930000$

H $-0.3675020000-1.73901000002 .3748130000$

H $1.0763980000-0.92026900001 .8280960000$

$\mathrm{H}-2.4996680000-1.69181600001 .2284220000$

H - $1.6844220000-2.5118620000-0.1019360000$

H - 2.6889540000 - $1.0953870000-0.4192580000$

H $1.24343400001 .6525120000-1.0256910000$

\section{Thermodynamic Data}

Zero-point correction $=0.259949$ (Hartree/Particle)

Thermal correction to Energy $=0.271719$

Thermal correction to Enthalpy $=0.272663$

Thermal correction to Gibbs Free Energy $=0.224007$

Sum of electronic and zero-point Energies $=-500.252758$

Sum of electronic and thermal Energies $=-500.240988$

Sum of electronic and thermal Enthalpies $=-500.240044$

Sum of electronic and thermal Free Energies $=-500.288700$

\begin{tabular}{llrr} 
& E (Thermal) & CV & \multicolumn{1}{c}{ S } \\
KCal/Mol & Cal/Mol-Kelvin & Cal/Mol-Kelvin \\
Total & 170.506 & 48.111 & 102.405
\end{tabular}




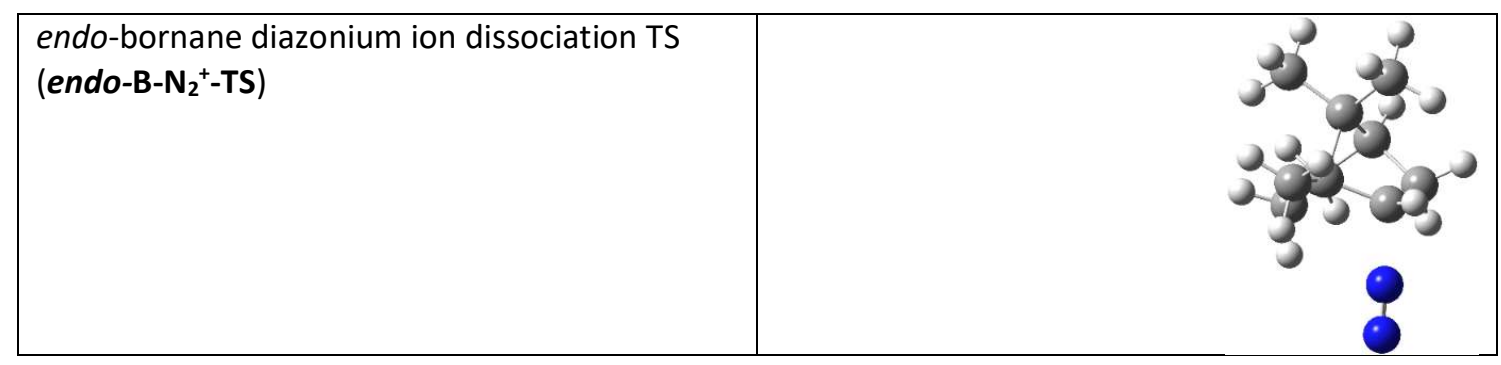

optimized xyz-matrix

C $0.8064010000-1.41926600000 .2412380000$

C - $0.4614860000-1.29716800001 .1275970000$

C -0.86586000000 .14772700000 .8192370000$

C $-0.02306400000 .6745270000-0.3095720000$

C $-0.2603190000-0.2107480000-1.5701820000$

C $0.3308050000-1.6082130000-1.2132530000$

H - $0.4054420000-2.4127310000-1.3011170000$

H $1.1615830000-1.8690750000-1.8724540000$

H $0.24673500000 .2460050000-2.4230680000$

H - $1.3220960000-0.2505640000-1.8384980000$

C 1.36022200000 .04218400000 .2596320000

C $2.53369300000 .3006890000-0.6998010000$

C 1.79251700000 .55218700001 .6501660000

C - $0.09276400002 .1778540000-0.5347700000$

H $-0.2675270000-1.39623200002 .1985430000$

H - $1.2292480000-2.03218200000 .8705290000$

H $1.4955220000-2.18800600000 .5983150000$

$\mathrm{N}-2.56816200000 .13095200000 .3045680000$

N $-3.62574700000 .1639650000-0.0103830000$

H - $1.06824300002 .4770060000-0.9368510000$

H $0.66078000002 .4877890000-1.2641330000$

H 0.07903600002 .74128600000 .3887990000

H 2.01699600001 .62337700001 .6171300000

H 2.71146900000 .03996800001 .9530810000

H 1.06482300000 .39381400002 .4527340000

H $3.3951870000-0.2903790000-0.3687330000$

H $2.83310300001 .3531720000-0.6720210000$

H 2.3317650000 0.0356900000 - 1.7385680000

H - 1.06794400000 .81800600001 .6552600000

Thermodynamic Data

Zero-point correction $=0.257701$ (Hartree/Particle)

Thermal correction to Energy $=0.269873$

Thermal correction to Enthalpy $=0.270817$

Thermal correction to Gibbs Free Energy $=0.220691$

Sum of electronic and zero-point Energies $=-500.250895$

Sum of electronic and thermal Energies $=-500.238723$

Sum of electronic and thermal Enthalpies $=-500.237779$

Sum of electronic and thermal Free Energies $=-\mathbf{5 0 0} .287905$

E (Thermal) CV S 


$\begin{array}{llrr} & \mathrm{KCal} / \mathrm{Mol} & \mathrm{Cal} / \mathrm{Mol}-\mathrm{Kelvin} & \text { Cal/Mol-Kelvin } \\ \text { Total } & 169.348 & 47.901 & 105.500\end{array}$

\begin{tabular}{|l|l|l|}
\hline $\begin{array}{l}\text { exo-bornane diazonium ion dissociation TS } \\
\text { (exo-B- } \mathrm{N}_{2}{ }^{+} \text {-TS) }\end{array}$ &
\end{tabular}

optimized xyz-matrix

C - $1.0862610000-0.7067700000-1.0712280000$

C $0.2689040000-1.4712500000-1.0400020000$

C $0.8686400000-0.96028400000 .2768280000$

C -0.06660100000 .11177000000 .8107290000$

C - $1.2222240000-0.84377500001 .3305740000$

C $-1.9566290000-1.31962900000 .0446890000$

H - $2.0114940000-2.4097020000-0.0275760000$

H - 2.9816560000 - 0.94537100000 .0055130000

H - $1.8614810000-0.24807400001 .9872030000$

H - $0.8402420000-1.66734700001 .9441540000$

C $-0.70862900000 .6990330000-0.5063230000$

C - $1.91695800001 .6135150000-0.2296890000$

C $0.25643600001 .4984640000-1.4030120000$

C 0.48714100001 .04921100001 .8737850000

H $0.8944540000-1.2307310000-1.9023810000$

H $0.1650790000-2.5597270000-1.0121430000$

H - $1.5390820000-0.7104470000-2.0652680000$

N 2.3970270000 -0.2881270000 -0.0455500000

N $3.41961900000 .1130510000-0.1592500000$

H 0.89130700000 .49825100002 .7308330000

$\mathrm{H}-0.30877100001 .69812200002 .2492730000$

H 1.27828000001 .69797800001 .4798530000

H $0.63642100002 .3841100000-0.8820580000$

H - $0.27820500001 .8553250000-2.2892520000$

H $1.11598400000 .9329290000-1.7765450000$

H - 2.4443500000 $1.8208510000-1.1666500000$

H - 1.58475400002 .57598400000 .1738520000

H - 2.6439110000 1.19652000000 .4698900000

H $1.2529840000-1.68485800000 .9967870000$

Thermodynamic Data

Zero-point correction $=0.258207$ (Hartree/Particle)

Thermal correction to Energy $=0.270075$

Thermal correction to Enthalpy $=0.271019$

Thermal correction to Gibbs Free Energy $=0.221848$

Sum of electronic and zero-point Energies $=-500.251934$

Sum of electronic and thermal Energies $=-500.240067$

Sum of electronic and thermal Enthalpies $=-500.239123$

Sum of electronic and thermal Free Energies $=-500.288294$

E (Thermal)

CV 


$\begin{array}{llrr} & \mathrm{KCal} / \mathrm{Mol} & \mathrm{Cal} / \mathrm{Mol}-\mathrm{Kelvin} & \mathrm{Cal} / \mathrm{Mol}-\mathrm{Kelvin} \\ \text { Total } & 169.475 & 47.479 & 103.490\end{array}$

\begin{tabular}{|l|l|l|}
\hline Bornyl cation $\left(\mathbf{B}^{+}\right)$ & & \\
\hline
\end{tabular}

\section{optimized xyz-matrix}

C $1.3740030000-0.4676500000-1.3028610000$

H $2.3714880000-0.0425230000-1.5019290000$

H $1.2548740000-1.2648960000-2.0478510000$

C $0.30545300000 .5453210000-1.4832900000$

H $-0.05011700000 .8382510000-2.4738800000$

C - $0.33172800000 .9387220000-0.2880570000$

C - $1.60684200001 .7540510000-0.3501780000$

H - $2.28060300001 .4085450000-1.1402190000$

H - 2.1414300000 1.70762500000 .6030650000

H - $1.37672400002 .8089090000-0.5384610000$

C 0.72903900001 .38779300000 .7921120000

H 1.26461400002 .26043100000 .4093890000

H 0.16927900001 .71789500001 .6704180000

C 1.65965500000 .18430800001 .0814370000

H 2.70743200000 .41160800000 .8678120000

H $1.6088720000-0.11846000002 .1299800000$

C $1.1250220000-0.91775400000 .1504030000$

H $1.5074300000-1.91691700000 .3740650000$

C - $0.4276070000-0.79658500000 .2331710000$

C $-1.2425580000-1.6984020000-0.6978600000$

H - $0.8462930000-1.7961900000-1.7111090000$

H - $1.2783900000-2.7038010000-0.2626760000$

H - $2.2767280000-1.3472960000-0.7717910000$

C - $1.0696310000-0.82121000001 .6138090000$

H $-0.6308540000-0.14133100002 .3430160000$

H -2.1449760000 -0.6308910000 1.5560600000

H - $0.9467030000-1.84252600002 .0019980000$

\section{Thermodynamic Data}

Zero-point correction $=0.247449$ (Hartree/Particle)

Thermal correction to Energy $=0.257371$

Thermal correction to Enthalpy $=0.258315$

Thermal correction to Gibbs Free Energy $=0.213820$

Sum of electronic and zero-point Energies $=-390.750682$

Sum of electronic and thermal Energies $=-390.740760$

Sum of electronic and thermal Enthalpies $=-390.739816$

Sum of electronic and thermal Free Energies $=-\mathbf{3 9 0 . 7 8 4 3 1 2}$

\begin{tabular}{llrr} 
& E (Thermal) & CV & \multicolumn{1}{c}{ S } \\
KCal/Mol & Cal/Mol-Kelvin & Cal/Mol-Kelvin \\
Total & 161.503 & 40.596 & 93.649
\end{tabular}




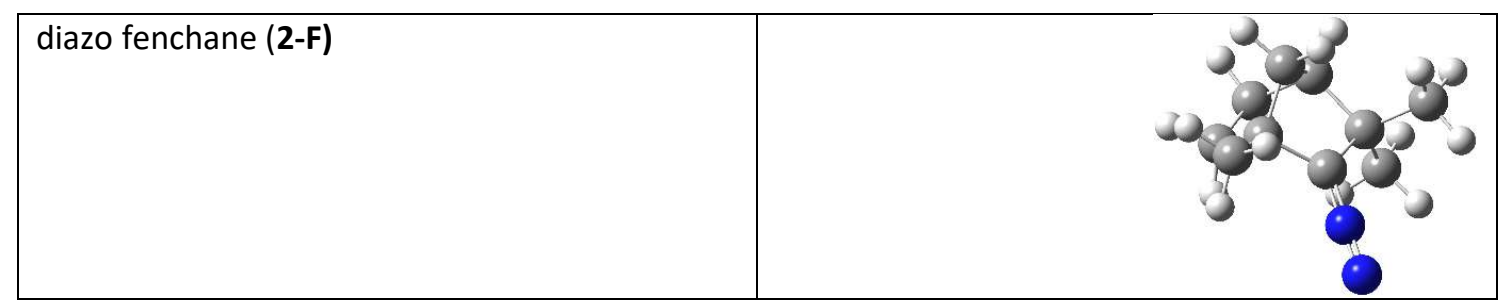

\section{optimized xyz-matrix}

C 0.20806800001 .59921100000 .3476730000

C 1.18424100000 .38537800000 .1566680000

C $0.1218640000-0.71563800000 .0134940000$

C - $1.2808600000-0.12832200000 .1860340000$

C - $1.56986400000 .7251440000-1.0888130000$

C $-0.56790000001 .9163900000-0.9576140000$

H $0.07859600002 .0322990000-1.8308130000$

H - $1.10574500002 .8633070000-0.8361870000$

$\mathrm{H}-2.60978000001 .0763550000-1.0822320000$

H - $1.42677300000 .1416380000-2.0038060000$

C - 0.93149700000 .99393800001 .1985560000

$\mathrm{H}-1.76279300001 .69408000001 .3480060000$

H - 0.62017000000 .60736600002 .1731920000

C - $2.3724360000-1.11618300000 .5681910000$

C 2.06976800000 .18433600001 .4068070000

C 2.1109800000 0.4966030000 -1.0689610000

H 0.71768900002 .46928800000 .7741600000

N $0.3738260000-1.9339540000-0.3035860000$

N $0.6089930000-3.0334060000-0.5736350000$

$\mathrm{H}-2.1263090000-1.64217600001 .4976000000$

H - 2.5220440000 - $1.8713710000-0.2132550000$

H -3.3266480000 -0.59637700000 .7120250000$

H $2.7825350000-0.3688720000-1.1165740000$

H $2.73717000001 .3952040000-1.0026780000$

H $1.55795700000 .5314360000-2.0112900000$

H 2.79304700001 .00444900001 .5005830000

H $2.6353350000-0.75161400001 .3301360000$

H 1.48401600000 .14135700002 .3294690000

\section{Thermodynamic Data}

Zero-point correction $=0.247385$ (Hartree/Particle) Thermal correction to Energy $=0.259102$

Thermal correction to Enthalpy $=0.260046$

Thermal correction to Gibbs Free Energy= 0.210805

Sum of electronic and zero-point Energies $=-499.894554$

Sum of electronic and thermal Energies $=-499.882838$

Sum of electronic and thermal Enthalpies $=-499.881894$

Sum of electronic and thermal Free Energies $=-499.931135$

\begin{tabular}{llrr} 
& E (Thermal) & CV & \multicolumn{1}{c}{ S } \\
KCal/Mol & Cal/Mol-Kelvin & Cal/Mol-Kelvin \\
Total & 162.589 & 46.763 & \multicolumn{1}{c}{103.637}
\end{tabular}




\begin{tabular}{|l|l|l|}
\hline $\begin{array}{l}\text { Endo-diazo fenchane dissociation TS } \\
\text { (endo-TS-2-F) }\end{array}$ & \\
\hline
\end{tabular}

\section{optimized xyz-matrix}

C $1.5215160000-0.72742000000 .3113810000$

C $1.05784600000 .6677270000-0.2182320000$

C $-0.37325300000 .3932720000-0.7252580000$

C $-0.6607580000-1.0434680000-0.3328430000$

C - $0.6623300000-1.23731200001 .2242950000$

C $0.8238060000-1.05634900001 .6486660000$

H $0.9625230000-0.28387300002 .4096660000$

H $1.2282830000-1.98618600002 .0639800000$

H - $1.0182700000-2.25095900001 .4475930000$

H - $1.3444990000-0.53891000001 .7151060000$

C $0.7486910000-1.6530740000-0.6516820000$

H $0.7830290000-2.7145650000-0.3776720000$

H $1.0401270000-1.5649000000-1.7023280000$

C - $1.8647140000-1.6651310000-1.0331210000$

C $1.88173000001 .0707730000-1.4744520000$

C 1.17758400001 .83508100000 .7779910000

H 2.6094340000 -0.8529270000 0.3366590000

$\mathrm{N}-1.69040100001 .51476800000 .2952400000$

N - 2.53077200002 .22681800000 .1148340000

H - $1.9375020000-2.7399240000-0.8246770000$

$\mathrm{H}-1.8006520000-1.5254480000-2.1173160000$

H - 2.7971420000 -1.1960530000 -0.6965870000

H $1.48290600001 .9955080000-1.9020610000$

H $1.86003300000 .3112170000-2.2606720000$

H 2.9299200000 $1.2331990000-1.1923100000$

H 0.82812500002 .76519900000 .3176210000

H 2.2272270000 1.97624900001 .0637850000

H 0.59395300001 .67669300001 .6882210000

\section{Thermodynamic Data}

Zero-point correction $=0.243421$ (Hartree/Particle)

Thermal correction to Energy $=0.255531$

Thermal correction to Enthalpy $=0.256475$

Thermal correction to Gibbs Free Energy $=0.206124$

Sum of electronic and zero-point Energies $=-499.838809$

Sum of electronic and thermal Energies $=-499.826699$

Sum of electronic and thermal Enthalpies $=-499.825754$

Sum of electronic and thermal Free Energies $=-499.876106$
E (Thermal)
CV
$S$
$\mathrm{KCal} / \mathrm{Mol} \quad \mathrm{Cal} / \mathrm{Mol}-\mathrm{Kelvin} \quad \mathrm{Cal} / \mathrm{Mol}-K e l v i n$ 


\begin{tabular}{|l|l|}
\hline $\begin{array}{l}\text { Exo-diazo fenchane dissociation TS } \\
\text { (exo-TS-2-F) }\end{array}$ \\
\hline
\end{tabular}

\section{optimized xyz-matrix}

C $1.3866970000-0.41420300000 .8344570000$

C $0.3035700000-1.1695540000-0.0206660000$

C $-0.5147280000-0.0191000000-0.6571600000$

C $0.04471100001 .2254550000-0.0100590000$

C $1.40450900001 .3255440000-0.8215180000$

C $2.35506800000 .3240760000-0.1119360000$

H $2.8801550000-0.3369900000-0.8058910000$

H 3.11760900000 .85327600000 .4703860000

H $1.75973600002 .3598760000-0.7617140000$

H $1.25456100001 .0976300000-1.8830210000$

C 0.57373800000 .77374500001 .3794090000

H 1.19801800001 .52825700001 .8751950000

H - 0.23702200000 .49701700002 .0617000000

C - $0.79665800002 .4920800000-0.0874090000$

C $-0.5310100000-2.09831200000 .8885600000$

C $0.8782150000-2.0456580000-1.1576870000$

H $1.8797990000-1.05246500001 .5752790000$

N - 2.3470010000 -0.1136080000 -0.0139850000

$\mathrm{N}-3.4072290000-0.1588620000-0.3620110000$

$\mathrm{H}-1.70246300002 .40482800000 .5236470000$

H -1.1076980000 2.6913350000 -1.1188620000

H -0.23269500003 .35988700000 .2756220000$

H - $0.9279140000-1.58062000001 .7657310000$

H $0.0987490000-2.92572900001 .2374650000$

H - $1.3740970000-2.52783200000 .3377420000$

H $0.0690590000-2.5955430000-1.6491520000$

H $1.5975890000-2.7726330000-0.7584430000$

H 1.3815550000 - $1.4574490000-1.9296660000$

\section{Thermodynamic Data}

Zero-point correction $=0.243682$ (Hartree/Particle)

Thermal correction to Energy $=0.255583$

Thermal correction to Enthalpy $=0.256527$

Thermal correction to Gibbs Free Energy $=0.207053$

Sum of electronic and zero-point Energies $=-499.843409$

Sum of electronic and thermal Energies $=-499.831508$

Sum of electronic and thermal Enthalpies $=-499.830564$

Sum of electronic and thermal Free Energies $=-499.880038$

\begin{tabular}{llrr} 
& E (Thermal) & CV & \multicolumn{1}{c}{ S } \\
KCal/Mol & Cal/Mol-Kelvin & Cal/Mol-Kelvin \\
Total & 160.381 & 47.240 & \multicolumn{2}{c}{104.127}
\end{tabular}




\begin{tabular}{|l|l|}
\hline $\begin{array}{l}\text { endo-fenchane diazonium ion } \\
\left.\text { (endo-F- } \mathrm{N}_{2}{ }^{+}\right)\end{array}$ & \\
\hline
\end{tabular}

optimized xyz-matrix

C $-0.5366880000-1.5174630000-0.2513720000$

C - $1.2715240000-0.21554500000 .2147830000$

C 0.01968400000 .64217500000 .6579900000

C $1.2880210000-0.22527900000 .2843890000$

C $1.3529700000-0.3663640000-1.2603900000$

C $0.1694850000-1.3197800000-1.6113290000$

H - $0.4817220000-0.9326760000-2.3971950000$

H $0.5460220000-2.2840850000-1.9643080000$

H 2.3180070000 - $0.8062100000-1.5282610000$

H $1.30831700000 .5969080000-1.7830570000$

C $0.6798350000-1.59282300000 .6936910000$

H $1.3663380000-2.40736000000 .4423690000$

H $0.4408520000-1.66702400001 .7588710000$

C 2.56575300000 .23380700000 .9715480000

C - $2.1323800000-0.45954700001 .4678990000$

C - $2.13849900000 .4586940000-0.8586390000$

H - $1.2153640000-2.3740670000-0.2248790000$

N 0.06540800001 .93327100000 .0349340000

N $0.11510200002 .9040610000-0.4996460000$

H 2.44585300000 .29761400002 .0588960000

H 2.90532300001 .21012200000 .6049200000

H $3.3683650000-0.48190200000 .7674260000$

H - $2.67617700001 .3231880000-0.4528130000$

H - 2.8941560000 - $0.2545800000-1.2020690000$

H - $1.58064700000 .7827660000-1.7421390000$

H - $2.9863370000-1.09077400001 .2019340000$

H - 2.52965800000 .48053600001 .8670760000

H - $1.5835400000-0.96525900002 .2677700000$

H 0.00500900000 .88422700001 .7270060000

\section{Thermodynamic Data}

Zero-point correction $=0.259529$ (Hartree/Particle)

Thermal correction to Energy $=0.271338$

Thermal correction to Enthalpy $=0.272282$

Thermal correction to Gibbs Free Energy $=0.223348$

Sum of electronic and zero-point Energies $=-500.258763$

Sum of electronic and thermal Energies $=-500.246955$

Sum of electronic and thermal Enthalpies $=-500.246011$

Sum of electronic and thermal Free Energies $=-500.294945$
E (Thermal)
CV
$\mathrm{KCal} / \mathrm{Mol}$
Cal/Mol-Kelvin
$\mathrm{S}$
Cal/Mol-Kelvin 
exo-fenchane diazonium ion $\left(\right.$ exo- $\left.-\mathrm{F}-\mathrm{N}_{2}{ }^{+}\right)$

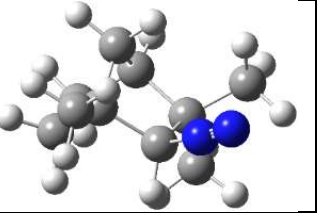

optimized xyz-matrix

C - $0.3128220000-1.29524100000 .8645690000$

C $-1.2145210000-0.3566080000-0.0109560000$

C $-0.06653500000 .5629810000-0.6632620000$

C $1.29382400000 .0861750000-0.0065230000$

C $1.5441940000-1.2929520000-0.6940370000$

C $0.5229110000-2.2516320000-0.0138050000$

H - $0.0697420000-2.8283440000-0.7265860000$

H $1.0374940000-2.97396300000 .6263080000$

H $2.5778040000-1.5904060000-0.4933230000$

H $1.4398460000-1.2410240000-1.7834600000$

C $0.7832270000-0.34493300001 .3865460000$

H $1.5618370000-0.86457200001 .9533080000$

H 0.42083000000 .48521500002 .0025470000

C $2.45334100001 .0680780000-0.0985680000$

C - 2.20016700000 .42655800000 .8764980000

C - $2.0022690000-1.0502010000-1.1327790000$

H - $0.9100780000-1.80027000001 .6278670000$

N $-0.28709800001 .9618710000-0.3723500000$

N $-0.43387200003 .0239660000-0.0899620000$

H 2.2574700000 2.00610100000 .4342480000

H 2.7094310000 $1.3045820000-1.1377870000$

H 3.33954400000 .62103900000 .3620190000

H - $2.6677060000-0.3432440000-1.6402080000$

H - 2.6271200000 - $1.8411980000-0.7050660000$

H - $1.3586040000-1.5079400000-1.8889770000$

H - $2.9271680000-0.27853900001 .2898390000$

H - 2.7694760000 1.16765800000 .3034060000

H - 1.72300400000 .92853800001 .7242100000

H - $0.05167300000 .5421580000-1.7582560000$

\section{Thermodynamic Data}

Zero-point correction $=0.259716$ (Hartree/Particle)

Thermal correction to Energy $=0.271439$

Thermal correction to Enthalpy $=0.272383$

Thermal correction to Gibbs Free Energy= 0.223691

Sum of electronic and zero-point Energies $=-500.258447$

Sum of electronic and thermal Energies $=-500.246724$

Sum of electronic and thermal Enthalpies $=-500.245780$

Sum of electronic and thermal Free Energies $=-500.294472$

\begin{tabular}{llrr} 
& E (Thermal) & CV & \multicolumn{1}{c}{ S } \\
KCal/Mol & Cal/Mol-Kelvin & Cal/Mol-Kelvin \\
Total & 170.331 & 48.018 & 102.481
\end{tabular}




\begin{tabular}{|l|l|l|}
\hline $\begin{array}{l}\text { endo-fenchane diazonium ion dissociation TS } \\
\left.\text { (endo-F- } \mathrm{N}_{2}{ }^{-}-\mathrm{TS}\right)\end{array}$ & \\
\hline
\end{tabular}

\section{optimized xyz-matrix}

C $0.7698030000-1.46180000000 .3279790000$

C $1.2814830000-0.0778180000-0.2113850000$

C - $0.07736400000 .4719180000-0.7130970000$

C $-1.2085100000-0.4314880000-0.2997490000$

C - $1.2710800000-0.53285800001 .2552150000$

C $0.0193480000-1.30418800001 .6650020000$

H $0.5983380000-0.79665500002 .4390530000$

H - $0.2322030000-2.29325400002 .0588010000$

H - 2.1770030000 - 1.08066300001 .5287160000

H -1.35214100000 .45447800001 .7241940000$

C - $0.4042450000-1.7506070000-0.6338220000$

H - $1.0121590000-2.6169680000-0.3539860000$

H $-0.1313890000-1.8555210000-1.6879650000$

C - $2.5364490000-0.2038340000-1.0085410000$

C $2.2084880000-0.2581430000-1.4368390000$

C 2.01109400000 .79844900000 .8230230000

H $1.5686320000-2.20646800000 .3481130000$

$\mathrm{N}-0.38580300002 .09039700000 .0101530000$

$\mathrm{N}-0.65311400003 .10279100000 .3592590000$

H - 2.4171330000 - $0.1622210000-2.0969080000$

H -3.0133190000 $0.7274390000-0.6805740000$

H -3.2287180000 -1.0192470000 -0.7787880000

H 2.37875800001 .72355300000 .3660630000

H 2.88222900000 .25379300001 .1991440000

H 1.38944000001 .05872900001 .6842410000

H $3.1448870000-0.7222830000-1.1122540000$

H $2.45421500000 .7086820000-1.8901360000$

H $1.7751730000-0.8974930000-2.2114260000$

H - $0.11059100000 .8739990000-1.7288970000$

\section{Thermodynamic Data}

Zero-point correction $=0.257595$ (Hartree/Particle)

Thermal correction to Energy $=0.269710$

Thermal correction to Enthalpy $=0.270654$

Thermal correction to Gibbs Free Energy $=0.220569$

Sum of electronic and zero-point Energies $=-500.254527$

Sum of electronic and thermal Energies $=-500.242412$

Sum of electronic and thermal Enthalpies $=-500.241468$

Sum of electronic and thermal Free Energies $=-500.291553$

$$
\begin{array}{lcc}
\text { E (Thermal) } & \text { CV } & \text { S } \\
\text { KCal/Mol } & \text { Cal/Mol-Kelvin } & \text { Cal/Mol-Kelvin } \\
& & 119
\end{array}
$$




\begin{tabular}{|l|l|}
\hline $\begin{array}{l}\text { exo-fenchane diazonium ion dissociation TS } \\
\text { (exo-F-N }{ }^{+} \text {-TS) }\end{array}$ & \\
\hline
\end{tabular}

\section{optimized xyz-matrix}

C $1.2960000000-0.34545700000 .9022040000$

C $0.3550190000-1.1973660000-0.0358970000$

C - $0.4702010000-0.0429490000-0.6679450000$

C - $0.04521500001 .2658200000-0.0207390000$

C $1.37778900001 .4639230000-0.6887210000$

C 2.30133600000 .46338700000 .0587470000

H 2.9079230000 -0.1473730000 -0.6127470000

H 2.99279400000 .99305900000 .7202990000

H $1.66547900002 .5058360000-0.5196060000$

H $1.35874000001 .3183230000-1.7747780000$

C 0.35735700000 .77650400001 .3926880000

H 0.87973900001 .55298900001 .9587760000

H - 0.49263900000 .43671400001 .9941500000

C - $0.97010700002 .4658140000-0.1722570000$

C $-0.4747370000-2.18669700000 .8117520000$

C $1.0684020000-1.9912730000-1.1480800000$

H $1.7504880000-0.96786300001 .6762990000$

$\mathrm{N}-2.1312180000-0.2640020000-0.2975350000$

N $-3.2075070000-0.3139580000-0.0565080000$

H - 1.92768000002 .31752100000 .3400750000

H - $1.17239200002 .6953490000-1.2246560000$

H - 0.50098500003 .34774500000 .2733980000

H $0.3603720000-2.6354350000-1.6805410000$

H $1.8345300000-2.6349430000-0.7044730000$

H $1.5592070000-1.3515920000-1.8868380000$

H $0.1988190000-2.94673800001 .2173580000$

H - $1.2211580000-2.70859200000 .2023520000$

H - $0.9782730000-1.71118300001 .6587470000$

H - $0.6177430000-0.0483250000-1.7500310000$

\section{Thermodynamic Data}

Zero-point correction $=0.257991$ (Hartree/Particle)

Thermal correction to Energy $=0.269896$

Thermal correction to Enthalpy $=0.270840$

Thermal correction to Gibbs Free Energy= 0.221378

Sum of electronic and zero-point Energies $=-500.256723$

Sum of electronic and thermal Energies $=-500.244817$

Sum of electronic and thermal Enthalpies $=-500.243873$

Sum of electronic and thermal Free Energies $=-\mathbf{5 0 0 . 2 9 3 3 3 5}$

\begin{tabular}{llrr} 
& E (Thermal) & CV & \multicolumn{1}{c}{ S } \\
KCal/Mol & Cal/Mol-Kelvin & Cal/Mol-Kelvin \\
Total & 169.362 & 47.458 & \multicolumn{1}{c}{104.101} \\
& & & 120
\end{tabular}




\begin{tabular}{|l|l|}
\hline Fenchyl cation $\left(\mathbf{F}^{+}\right)$ &
\end{tabular}

\section{optimized xyz-matrix}

C $0.6026110000-0.30875900001 .3527620000$

H 1.45611700000 .06159500001 .9316400000

H $0.2833220000-1.26450300001 .7752770000$

C $0.0061980000-0.9373760000-0.5705410000$

H - $0.0587220000-1.9512520000-0.9786510000$

C $1.2594390000-0.3592610000-0.2217420000$

C $2.5275630000-1.1613350000-0.4431400000$

H $2.3800570000-2.2300660000-0.2598890000$

H $2.8785720000-1.0356520000-1.4732310000$

H $3.3240970000-0.80642000000 .2178140000$

C $1.36434500001 .1844280000-0.4491680000$

H $2.36148700001 .4928530000-0.1232770000$

H $1.29712200001 .3898140000-1.5212300000$ C 0.24126600001 .86034400000 .3796140000

H 0.66541900002 .47347600001 .1799200000

H - $0.39330400002 .5154000000-0.2207730000$

C -0.52687100000 .67557800000 .9826560000$

H -1.1991390000 0.9470330000 1.7993420000

C $-1.2159230000-0.2014680000-0.1193420000$

C -2.2430160000 -1.1871710000 0.4943040000

H -3.1160560000 -0.6186230000 0.8296000000

H -2.5786760000 -1.9178430000 -0.2487100000

H -1.8483190000 -1.7319460000 1.3568990000

C - $1.88734800000 .5274480000-1.3147720000$

H -1.2040630000 1.1884360000 -1.8523070000

H -2.2992400000 -0.1946620000 -2.0263880000

H -2.7182630000 $1.1277860000-0.9298190000$

\section{Thermodynamic Data}

Zero-point correction $=0.247837$ (Hartree/Particle)

Thermal correction to Energy $=0.257731$

Thermal correction to Enthalpy $=0.258675$

Thermal correction to Gibbs Free Energy $=0.214134$

Sum of electronic and zero-point Energies $=-390.750380$

Sum of electronic and thermal Energies $=-390.740486$

Sum of electronic and thermal Enthalpies $=-390.739542$

Sum of electronic and thermal Free Energies $=-390.784083$

\begin{tabular}{llrr} 
& E (Thermal) & CV & \multicolumn{1}{c}{ S } \\
KCal/Mol & Cal/Mol-Kelvin & Cal/Mol-Kelvin \\
Total & 161.729 & 40.442 & 93.744
\end{tabular}


4.2.4 Structures optimized at the B3LYP/6-311+G** level of theory

\begin{tabular}{|l|l|l|}
\hline Diazo norbornane (2-N) & \\
\hline
\end{tabular}

optimized xyz-matrix

C - $1.36282600000 .9538530000-0.0330050000$

C $0.08423800001 .3976030000-0.3699400000$

C 0.84567800000 .16985900000 .1127530000

C $-0.1457070000-0.80588600000 .7277840000$

C $-0.9692830000-1.4343400000-0.4280470000$

C $-1.7666460000-0.2059310000-0.9816190000$

$\mathrm{H}-1.51938900000 .0173510000-2.0223450000$

H - $-2.8440470000-0.3826090000-0.9354480000$

H -1.6440690000 -2.2045730000 -0.0414710000

H - $0.3304160000-1.9005130000-1.1800370000$

C - 1.16583000000 .20547800001 .3050080000

H - $2.0796510000-0.27537200001 .6646710000$

$\mathrm{H}-0.74981900000 .83111700002 .0979450000$

H $0.2927850000-1.52087800001 .4237000000$

H 0.36336000002 .30280900000 .1834770000

H $0.21882900001 .6020790000-1.4371980000$

H - $2.07196800001 .7822980000-0.0200250000$

N $2.0975130000-0.0464040000-0.0542910000$

N $3.2234350000-0.2300990000-0.1929760000$

\section{Thermodynamic Data}

Zero-point correction $=0.162107$ (Hartree/Particle)

Thermal correction to Energy $=0.169419$

Thermal correction to Enthalpy $=0.170363$

Thermal correction to Gibbs Free Energy $=0.130018$

Sum of electronic and zero-point Energies $=-382.136500$

Sum of electronic and thermal Energies $=-382.129188$

Sum of electronic and thermal Enthalpies $=-382.128244$

Sum of electronic and thermal Free Energies $=-\mathbf{3 8 2} .168589$

\begin{tabular}{rlrr} 
& E (Thermal) & CV & \multicolumn{1}{c}{ S } \\
& KCal/Mol & Cal/Mol-Kelvin & Cal/Mol-Kelvin \\
Total & 106.312 & 29.183 & 84.913
\end{tabular}

\begin{tabular}{|l|l|}
\hline endo-Norbornyl diazonium ion $\left(\right.$ endo- $\left.\mathbf{N}-\mathbf{N}_{2}{ }^{+}\right)$ & \\
\hline
\end{tabular}

optimized xyz-matrix

C $1.4839820000-0.5285950000-0.6421790000$ 
C $0.1445310000-1.3005520000-0.7334210000$

C - $0.5687760000-0.77953700000 .5189140000$

C 0.27837100000 .29344900001 .1329840000

C 0.43198100001 .50541600000 .1781230000

C $1.24610800000 .9451800000-1.0310440000$

H $0.71572200001 .0422750000-1.9802080000$

H 2.1948550000 $1.4718620000-1.1391380000$

H 0.97872900002 .28862200000 .7039450000

H - $0.53011800001 .9364750000-0.1079500000$

C $1.6480820000-0.44476100000 .8916740000$

H $0.2768820000-2.3800700000-0.6211480000$

H - $0.4065570000-1.1381070000-1.6611870000$

H 2.2889840000 -1.0183370000 -1.1862590000

$\mathrm{N}-2.1255420000-0.06137000000 .0042860000$

$\mathrm{N}-3.11352100000 .3244950000-0.2690720000$

H -1.0550840000 -1.4976620000 1.1766720000

H 2.46840900000 .19064700001 .2292950000

H $1.7335110000-1.41498500001 .3864760000$

H 0.02243600000 .53380800002 .1626980000

\section{Thermodynamic Data}

Zero-point correction $=0.172463$ (Hartree/Particle)

Thermal correction to Energy $=0.180221$

Thermal correction to Enthalpy $=0.181165$

Thermal correction to Gibbs Free Energy= 0.139882

Sum of electronic and zero-point Energies $=-\mathbf{3 8 2 . 4 8 9 5 5 7}$

Sum of electronic and thermal Energies $=-382.481798$

Sum of electronic and thermal Enthalpies $=-382.480854$

Sum of electronic and thermal Free Energies $=-\mathbf{3 8 2 . 5 2 2 1 3 8}$

\begin{tabular}{llrr} 
& E (Thermal) & CV & \multicolumn{1}{c}{ S } \\
KCal/Mol & Cal/Mol-Kelvin & Cal/Mol-Kelvin \\
Total & 113.091 & 30.149 & 86.889
\end{tabular}

\begin{tabular}{|l|l|l|}
\hline exo-Norbornyl diazonium $\left(\right.$ exo- $\left.\mathbf{N}-\mathbf{N}_{2}{ }^{+}\right)$ & \\
\hline
\end{tabular}

\section{optimized xyz-matrix}

C - 1.11315400001 .03385400000 .4279310000

C $0.03261200001 .2610240000-0.6000520000$

C $0.6254100000-0.1504560000-0.7201550000$

C - $0.1345810000-1.01254000000 .2614750000$

C - $-1.5202310000-1.1765220000-0.4653810000$

C $-2.21389100000 .2044430000-0.2624130000$

H - $2.54661400000 .6501380000-1.2011410000$

H -3.0879520000 0.11322600000 .3834960000

H - 2.0539400000 -1.9874940000 0.0320720000

H - $1.4188140000-1.4644990000-1.5143650000$

C - $-0.4782200000-0.00331600001 .3778070000$ 
H $0.76118000001 .9822160000-0.2250670000$

H - $0.29748100001 .6146720000-1.5787330000$

$\mathrm{H}-1.45244500001 .96224800000 .8831330000$

N 2.2399320000 -0.0865010000 -0.1788260000

N $3.2617990000-0.09204400000 .2167890000$

H $0.8514150000-0.5545250000-1.7050410000$

H -1.1846320000 -0.4145080000 2.0994880000

H 0.39305800000 .36831200001 .9249790000

H $0.3364390000-1.95890000000 .5201570000$

\section{Thermodynamic Data}

Zero-point correction $=0.172570$ (Hartree/Particle)

Thermal correction to Energy $=0.180235$

Thermal correction to Enthalpy $=0.181179$

Thermal correction to Gibbs Free Energy $=0.140188$

Sum of electronic and zero-point Energies $=-382.492619$

Sum of electronic and thermal Energies $=-382.484954$

Sum of electronic and thermal Enthalpies $=-382.484009$

Sum of electronic and thermal Free Energies $=-382.525000$

\begin{tabular}{llrr} 
& E (Thermal) & CV & \multicolumn{1}{c}{ S } \\
KCal/Mol & Cal/Mol-Kelvin & Cal/Mol-Kelvin \\
Total & 113.099 & 29.984 & 86.272
\end{tabular}

\begin{tabular}{|l|l|}
\hline Diazo bornane (2-B) & \\
\hline
\end{tabular}

optimized xyz-matrix

C - 0.95891100001 .22638900000 .5754690000

C 0.50677300001 .37254000001 .0556080000

C 1.14366600000 .25133900000 .2502470000

C $0.0633620000-0.4717080000-0.5599850000$

C $-0.33997400000 .5284350000-1.6839450000$

C - $1.00976700001 .7000470000-0.8998800000$

H $-0.47708000002 .6448870000-1.0301130000$

$\mathrm{H}-2.03667600001 .8680590000-1.2300380000$

H - $1.03507000000 .0632700000-2.3893530000$

H $0.53382900000 .8468290000-2.2558350000$

C $-1.1418100000-0.31990800000 .4510450000$

C - $2.5084290000-0.7332690000-0.1206260000$

C - $0.9431080000-1.08461200001 .7690440000$

C $0.4264570000-1.8673900000-1.0389630000$

H 0.60643900001 .22290100002 .1364650000

H 0.91506200002 .36086100000 .8207080000

H -1.6764660000 1.7268900000 1.2291720000

N 2.40085900000 .02062600000 .1669160000

N $3.5277360000-0.19807000000 .1000460000$

H $1.2873590000-1.8403400000-1.7140500000$ 
H $-0.4045130000-2.3158480000-1.5901020000$

H $0.6799970000-2.5266440000-0.2045250000$

$\mathrm{H}-1.0921820000-2.15731000001 .6150040000$

$\mathrm{H}-1.6773980000-0.75922000002 .5128070000$

H $0.0524030000-0.95211800002 .1944020000$

H -3.2968840000 -0.50476500000 .6035450000$

H - $2.5401780000-1.8118810000-0.3008310000$

H - $2.7683630000-0.2346550000-1.0540620000$

\section{Thermodynamic Data}

Zero-point correction $=0.245124$ (Hartree/Particle)

Thermal correction to Energy $=0.256808$

Thermal correction to Enthalpy $=0.257752$

Thermal correction to Gibbs Free Energy $=0.208756$

Sum of electronic and zero-point Energies $=-500.025524$

Sum of electronic and thermal Energies $=-500.013841$

Sum of electronic and thermal Enthalpies $=-500.012897$

Sum of electronic and thermal Free Energies $=-500.061892$

\begin{tabular}{llrr} 
& E (Thermal) & CV & \multicolumn{1}{c}{ S } \\
KCal/Mol & Cal/Mol-Kelvin & Cal/Mol-Kelvin \\
Total & 161.149 & 47.068 & 103.120
\end{tabular}

\begin{tabular}{|l|l|}
\hline $\begin{array}{l}\text { endo-bornane diazonium ion dissociation TS } \\
\text { (endo-B- } \mathbf{N}_{2}{ }^{+} \text {-TS) }\end{array}$ & \\
\hline
\end{tabular}

\section{optimized xyz-matrix}

C $0.8055770000-1.41479300000 .2510040000$

C - $0.4648530000-1.29120200001 .1304090000$

C - 0.87809100000 .14817500000 .8105770000

C $-0.02335600000 .6711230000-0.3140900000$

C $-0.2512810000-0.2177850000-1.5713200000$

C $0.3363190000-1.6128980000-1.2032330000$

H - $0.3983730000-2.4156440000-1.2879400000$

H $1.1669930000-1.8785470000-1.8562150000$

H $0.26347400000 .2324890000-2.4195880000$

H - $1.3072580000-0.2593690000-1.8517260000$

C 1.35581500000 .04680100000 .2616460000

C $2.53227800000 .2967590000-0.6932050000$

C 1.77951500000 .56457400001 .6499640000

C - $0.09713500002 .1716430000-0.5445720000$

$\mathrm{H}-0.2729740000-1.38093300002 .1999170000$

H -1.2248090000 -2.0315590000 0.8768330000

H $1.4938500000-2.17771400000 .6139260000$

$\mathrm{N}-2.55726600000 .13127900000 .2928140000$

N -3.6105310000 $0.1614870000-0.0071220000$

H - $1.07040800002 .4665090000-0.9496220000$

H $0.65469900002 .4814020000-1.2721400000$ 
H 0.07028400002 .73731600000 .3755380000

H 2.01058300001 .63156300001 .6096720000

H 2.69117900000 .05084000001 .9636350000

H 1.04660300000 .41749400002 .4464720000

H $3.3910470000-0.2891460000-0.3539630000$

H $2.83044000001 .3472510000-0.6728580000$

H 2.3356880000 0.0231880000 - 1.7280430000

H - 1.07517400000 .82111600001 .6431760000

\section{Thermodynamic Data}

Zero-point correction $=0.255437$ (Hartree/Particle)

Thermal correction to Energy $=0.267606$

Thermal correction to Enthalpy $=0.268550$

Thermal correction to Gibbs Free Energy $=0.218529$

Sum of electronic and zero-point Energies $=-\mathbf{5 0 0 . 3 8 2 5 4 6}$

Sum of electronic and thermal Energies $=-500.370377$

Sum of electronic and thermal Enthalpies $=-500.369433$

Sum of electronic and thermal Free Energies $=-500.419454$

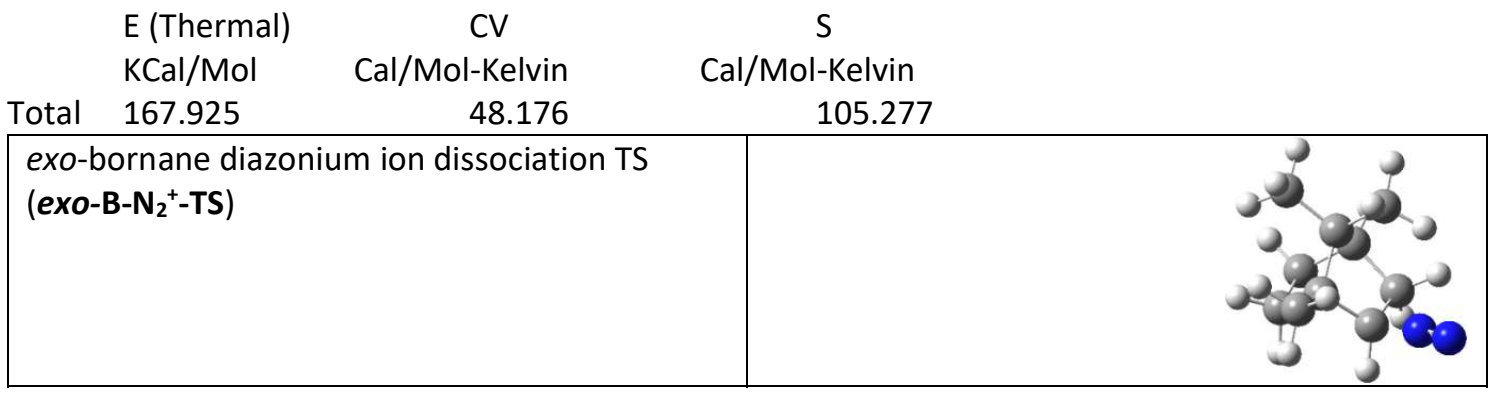

\section{optimized xyz-matrix}

C - $1.0822610000-0.7178870000-1.0640020000$

C $0.2774450000-1.4707640000-1.0348440000$

C $0.8822620000-0.94855800000 .2754310000$

C - 0.06409700000 .11746700000 .8090330000

C - $1.2116340000-0.83519200001 .3388820000$

C - $1.9434120000-1.32872300000 .0587610000$

H - $1.9879710000-2.4172260000-0.0049950000$

H - $2.9697440000-0.96591300000 .0189750000$

H - $1.8552500000-0.24094200001 .9877840000$

H - $0.8241160000-1.64879600001 .9578730000$

C - $0.71259400000 .6925730000-0.5093790000$

C - $1.92580500001 .5978900000-0.2342040000$

C $0.24355000001 .4908040000-1.4138410000$

C 0.48765100001 .06640200001 .8602990000

H $0.8947160000-1.2311780000-1.9001260000$

H $0.1799000000-2.5570830000-1.0005930000$

H - $1.5392270000-0.7301200000-2.0531850000$

N 2.3887110000 -0.2794600000 -0.0422090000

N $3.40521300000 .1134180000-0.1589640000$

H 0.90345200000 .52564000002 .7154830000

H - 0.31067600001 .70633200002 .2389500000

H 1.26562000001 .72225200001 .4584230000

H $0.62345800002 .3772480000-0.8993840000$ 
H - $0.29787500001 .8432380000-2.2947800000$

H $1.09860000000 .9277870000-1.7932910000$

$\mathrm{H}-2.45584300001 .7962090000-1.1688520000$

$\mathrm{H}-1.60071700002 .56289900000 .1624420000$

H - 2.6458850000 1.17929500000 .4673990000

H $1.2574560000-1.67142500000 .9992660000$

\section{Thermodynamic Data}

Zero-point correction $=0.255939$ (Hartree/Particle)

Thermal correction to Energy $=0.267803$

Thermal correction to Enthalpy $=0.268748$

Thermal correction to Gibbs Free Energy $=0.219664$

Sum of electronic and zero-point Energies $=-500.383290$

Sum of electronic and thermal Energies $=-500.371426$

Sum of electronic and thermal Enthalpies $=-500.370481$

Sum of electronic and thermal Free Energies $=-500.419565$

$\begin{array}{llrr} & \text { E (Thermal) } & \text { CV } & \text { S } \\ & \text { KCal/Mol } & \text { Cal/Mol-Kelvin } & \text { Cal/Mol-Kelvin } \\ \text { Total } & 168.049 & 47.760 & 103.306\end{array}$

diazo fenchane (2-F)

optimized xyz-matrix

C - $0.2141420000-1.59881000000 .3415000000$

C - $1.1849690000-0.37979300000 .1582430000$

C - 0.11889500000 .71746200000 .0201350000

C 1.28049800000 .12327900000 .1883040000

C $1.5637860000-0.7226720000-1.0916470000$

C $0.5580660000-1.9103090000-0.9663710000$

H - $0.0891170000-2.0171050000-1.8368310000$

H $1.0912310000-2.8578480000-0.8524850000$

H 2.5999530000 -1.0768220000 -1.0873590000

H $1.4214210000-0.1341190000-2.0005160000$

C $0.9275020000-1.00292500001 .1945510000$

H $1.7550260000-1.70475600001 .3369990000$

H $0.6185310000-0.62146400002 .1686860000$

C 2.37499100001 .10372600000 .5747310000

C - $2.0666410000-0.18230300001 .4106030000$

C - $2.1101040000-0.4804930000-1.0669710000$

H $-0.7266860000-2.46643300000 .7624660000$

$\mathrm{N}-0.36607600001 .9327960000-0.3008140000$

$\mathrm{N}-0.59720200003 .0254600000-0.5755870000$

H 2.1281080000 1.62976800001 .5010820000

H $2.53248000001 .8552770000-0.2053450000$

H 3.32312600000 .57872900000 .7222160000

H - $2.77879700000 .3844080000-1.1088150000$ 
H - $2.7366810000-1.3763580000-1.0057870000$

H - $1.5576820000-0.5112760000-2.0068860000$

H - 2.7873130000 -1.0018490000 1.5020620000

H - 2.6321490000 0.75114700001 .3377770000

H - $1.4790550000-0.14207000002 .3290760000$

\section{Thermodynamic Data}

Zero-point correction $=0.244830$ (Hartree/Particle) Thermal correction to Energy $=0.256613$

Thermal correction to Enthalpy $=0.257557$

Thermal correction to Gibbs Free Energy $=0.208174$

Sum of electronic and zero-point Energies $=-500.028260$

Sum of electronic and thermal Energies $=-500.016477$

Sum of electronic and thermal Enthalpies $=-500.015533$

Sum of electronic and thermal Free Energies $=-500.064916$

\begin{tabular}{llrr} 
& E (Thermal) & CV & \multicolumn{1}{c}{ S } \\
KCal/Mol & Cal/Mol-Kelvin & Cal/Mol-Kelvin \\
Total & 161.027 & 47.181 & 103.935
\end{tabular}

\begin{tabular}{|l|r|}
\hline $\begin{array}{l}\text { endo-fenchane diazonium dissociation TS } \\
\text { (endo-F-N }{ }_{2}^{+}-\mathrm{TS} \text { ) }\end{array}$ & \\
\hline
\end{tabular}

optimized xyz-matrix

C $0.7451050000-1.47712800000 .3028780000$

C $1.2771370000-0.0925110000-0.2125160000$

C - $0.07471500000 .4892340000-0.7007940000$

C $-1.2164600000-0.4063920000-0.2963980000$

C - $1.2734160000-0.53060900001 .2553710000$

C $0.0043480000-1.33103900001 .6449860000$

H $0.5940140000-0.84966500002 .4238060000$

H - $0.2621150000-2.32036700002 .0209050000$

H - 2.1847960000 -1.0654350000 1.5260130000

H - 1.33345400000 .44685900001 .7420290000

C - $0.4369560000-1.7306710000-0.6564030000$

H - $1.0532120000-2.5910660000-0.3877160000$

H - $0.1709390000-1.8210320000-1.7108980000$

C - $2.5420970000-0.1461300000-0.9944730000$

C $2.1977150000-0.2663850000-1.4411050000$

C 2.02028500000 .74883700000 .8379500000

H $1.5304440000-2.23234200000 .3062310000$

N -0.35126900002 .08910800000 .0256350000$

N -0.58765900003 .10079700000 .3725510000$

H - $2.4269150000-0.0918460000-2.0803490000$

H -3.0015020000 $0.7862600000-0.6520100000$

H -3.2463000000 -0.9505150000 -0.7734860000

H 2.40294800001 .67495000000 .4011510000

H 2.88108600000 .18397600001 .2010290000

H 1.40517300000 .99984400001 .7033740000 
H $3.1227670000-0.7555860000-1.1292630000$

H $2.46264700000 .7023040000-1.8737390000$

H $1.7502210000-0.8786190000-2.2260020000$

H - $0.10324900000 .8897140000-1.7153470000$

\section{Thermodynamic Data}

Zero-point correction $=0.255390$ (Hartree/Particle)

Thermal correction to Energy $=0.267479$

Thermal correction to Enthalpy $=0.268423$

Thermal correction to Gibbs Free Energy $=0.218508$

Sum of electronic and zero-point Energies $=-500.385893$

Sum of electronic and thermal Energies $=-500.373804$

Sum of electronic and thermal Enthalpies $=-500.372860$

Sum of electronic and thermal Free Energies $=-500.422775$

\begin{tabular}{llrr} 
& E (Thermal) & CV & \multicolumn{1}{c}{ S } \\
& KCal/Mol & Cal/Mol-Kelvin & Cal/Mol-Kelvin \\
Total & 167.846 & 48.014 & 105.056
\end{tabular}

\begin{tabular}{|l|l|}
\hline $\begin{array}{l}\text { exo-fenchane diazonium ion dissociation TS } \\
\left.\text { (exo-F- } \mathrm{N}_{2}{ }^{+}-\mathrm{TS}\right)\end{array}$ & \\
\hline
\end{tabular}

\section{optimized xyz-matrix}

C $1.2898070000-0.37185800000 .8968380000$

C $0.3259740000-1.2035190000-0.0346500000$

C - $0.4819010000-0.0322650000-0.6622050000$

C - $0.01876700001 .2669300000-0.0187010000$

C $1.40252100001 .4367410000-0.6907740000$

C 2.30696900000 .41477300000 .0489730000

H $2.8939140000-0.2076940000-0.6249000000$

H 3.01314800000 .92608100000 .7052530000

H $1.71530100002 .4684090000-0.5211940000$

H $1.37509000001 .2941040000-1.7745700000$

C 0.37784900000 .76833200001 .3918020000

H 0.91857200001 .53081000001 .9545910000

H - 0.47354100000 .44585600001 .9959170000

C - $0.91772300002 .4851750000-0.1638290000$

C - $0.5150270000-2.17537000000 .8186350000$

C $1.0165280000-2.0086660000-1.1505710000$

H $1.7342940000-1.00227900001 .6664700000$

$\mathrm{N}-2.1287600000-0.2163500000-0.2927800000$

N -3.1997910000 -0.2599570000 -0.0658540000

H - 1.87075300002 .36237300000 .3588200000

H - $1.12485200002 .7152300000-1.2128960000$

H - 0.42562600003 .35649700000 .2719660000

H $0.2930560000-2.6312690000-1.6833910000$

H $1.7655630000-2.6724520000-0.7132500000$

H $1.5206210000-1.3789900000-1.8851380000$

H $0.1468770000-2.94196600001 .2245090000$ 


\section{Thermodynamic Data}

Zero-point correction $=0.255690$ (Hartree/Particle)

Thermal correction to Energy $=0.267595$

Thermal correction to Enthalpy $=0.268539$

Thermal correction to Gibbs Free Energy $=0.219156$

Sum of electronic and zero-point Energies $=-500.387758$

Sum of electronic and thermal Energies $=-500.375853$

Sum of electronic and thermal Enthalpies $=-500.374908$

Sum of electronic and thermal Free Energies $=-\mathbf{5 0 0 . 4 2 4 2 9 1}$

\begin{tabular}{llrr} 
& E (Thermal) & CV & \multicolumn{1}{c}{ S } \\
& KCal/Mol & Cal/Mol-Kelvin & Cal/Mol-Kelvin \\
Total & 167.919 & 47.747 & 103.935
\end{tabular}

\subsection{Studies on the topology of the underlying PES}

4.3.1 IRC-Profiles, PES Scans and Activation Barriers based on Stationary Points In the following section one finds the summarized results of:

a) the activation barriers for the thermal dissociation of the diazoniumions $\mathrm{N}-\mathrm{N}_{2}{ }^{+}, \mathbf{B}-\mathrm{N}_{2}{ }^{+}, \mathbf{F}-\mathrm{N}_{2}{ }^{+}$, in dependence of their configuration at $\mathrm{C} 2$ (endo, exo) as calculated at various levels of theory with and without implicit solvent modelling.

b) The relaxed PES scans in the region of the N2-dissociation and potential skeletal rearrangements of the resulting carbocations.

c) IRC calculations starting at the respective transition states that indicate the dinitrogen release.

\subsubsection{Norbornyl system}

Table $\mathbf{5 4 2}$ Activation barriers and driving forces for the thermal dissociation to the indicated product structures $\mathbf{N}^{+}$and nor- $\mathbf{P}^{+}$ (see below, exemplified for the endo-configuration).

\begin{tabular}{lllll}
\hline Level of theory & \multicolumn{2}{c}{ endo [kcal/mol] } & \multicolumn{2}{c}{ exo [kcal/mol] } \\
\hline & $\Delta^{\ddagger} G\left(\Delta^{\ddagger} H\right)$ & $\Delta_{R} G\left(\Delta_{R} H\right)$ & $\Delta^{\ddagger} G\left(\Delta^{\ddagger} H\right)$ & $\Delta_{R} G\left(\Delta_{R} H\right)$ \\
\cline { 2 - 6 } B3LYP/6-31G*, Gasphase & $1.7(2.3)$ & $25.8(14.8)$ & $0.3(0.7)$ & $25.3(14.3)$ \\
M062X/6-311+G**, Gasphase & $1.4(2.4)$ & $26.0(15.4)$ & $0.2(0.6)$ & $25.2(14.7)$ \\
M062X/6-311+G**, PCM=ACN & $3.0(3.9)$ & $25.9(15.2)$ & $1.4(2.0)$ & $25.0(14.4)$ \\
BB1K/6-31+G(d,p), Gasphase & $34.8(37.4)$ & $28.5(16.2)$ & $32.8(35.1)$ & $28.1(15.8)$
\end{tabular}



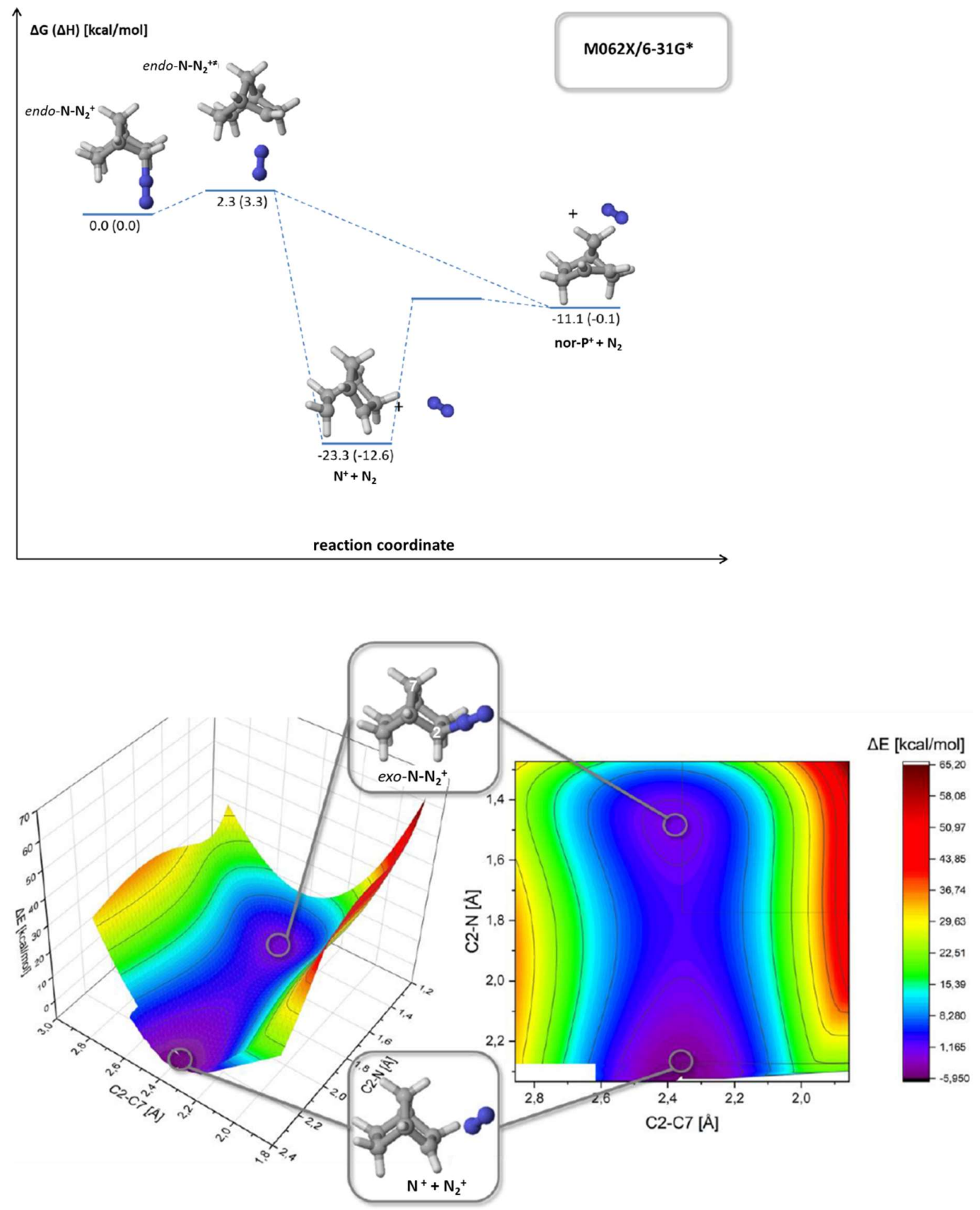

Figure S19. Relaxed PES scan driving the bond lengths $\mathrm{C} 2-\mathrm{N}_{2}$ (coordinate for the $\mathrm{N}_{2}$-release) and $\mathrm{C} 2-\mathrm{C} 7$ (norbornyl vs norpinyl cation rearrangement) to learn more about the topology in this region and dimension of the PES. 


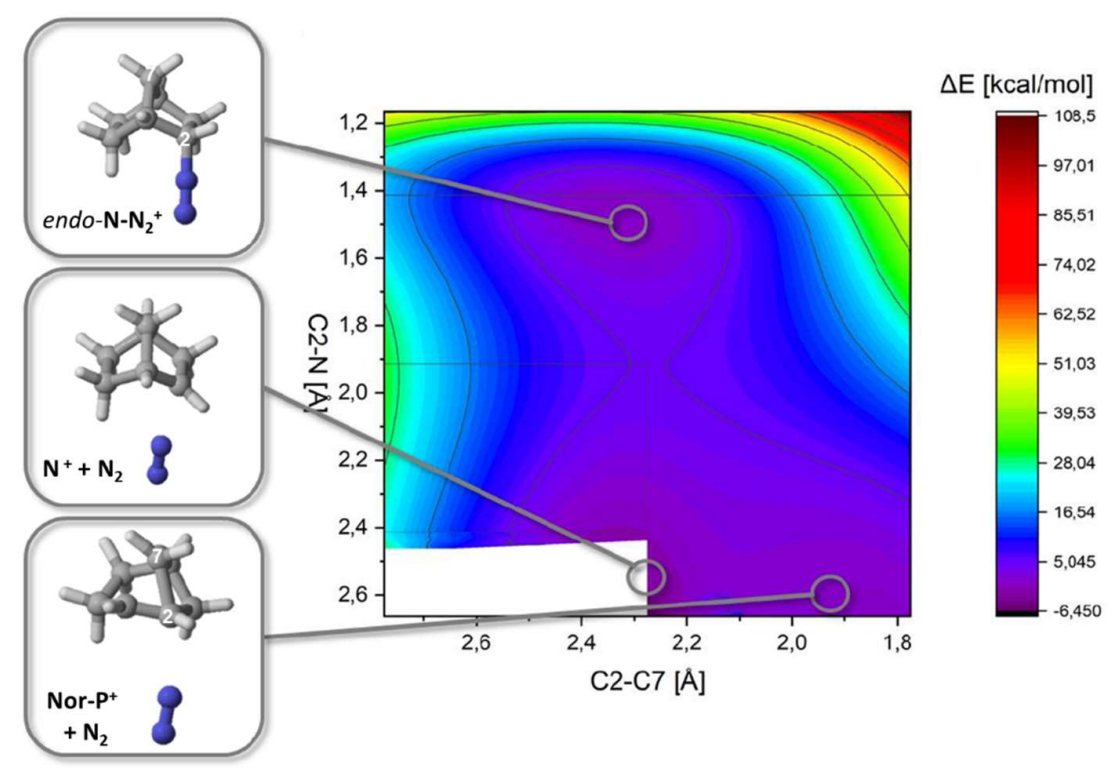

Figure S20. Relaxed PES scan driving the bond lengths $\mathrm{C} 2-\mathrm{N}_{2}$ (coordinate for the $\mathrm{N}_{2}$-release) and $\mathrm{C} 2-\mathrm{C} 7$ (norbornyl vs norpinyl cation rearrangement) to learn more about the topology in this region and dimension of the PES.

\subsubsection{Bornyl system}

Table S43. Activation barriers and driving forces for the thermal dissociation to the indicated product structures $\mathbf{C}^{+}, \mathbf{P}^{+}$and $\mathrm{T}^{+}$(see below, exemplified for the endo-configuration).

\begin{tabular}{lcc}
\hline Level of theory & endo [kcal/mol] & exo [kcal/mol] \\
\hline & $\Delta^{\ddagger} G\left(\Delta^{\ddagger} H\right)$ & $\Delta^{\ddagger} G\left(\Delta^{\ddagger} H\right)$ \\
\cline { 2 - 3 } B3LYP/6-31G*, Gasphase & $1.4(2.2)$ & $0.3(0.6)$ \\
M062X/6-311+G**, Gasphase & $1.9(2.2)$ & $0.5(0.5)$ \\
M062X/6-311+G**, PCM=ACN & $3.1(3.9)$ & $1.4(1.3)$ \\
BB1K/6-31+G(d,p), Gasphase & $33.4(36.7)$ & $31.7(34.2)$ \\
\hline
\end{tabular}

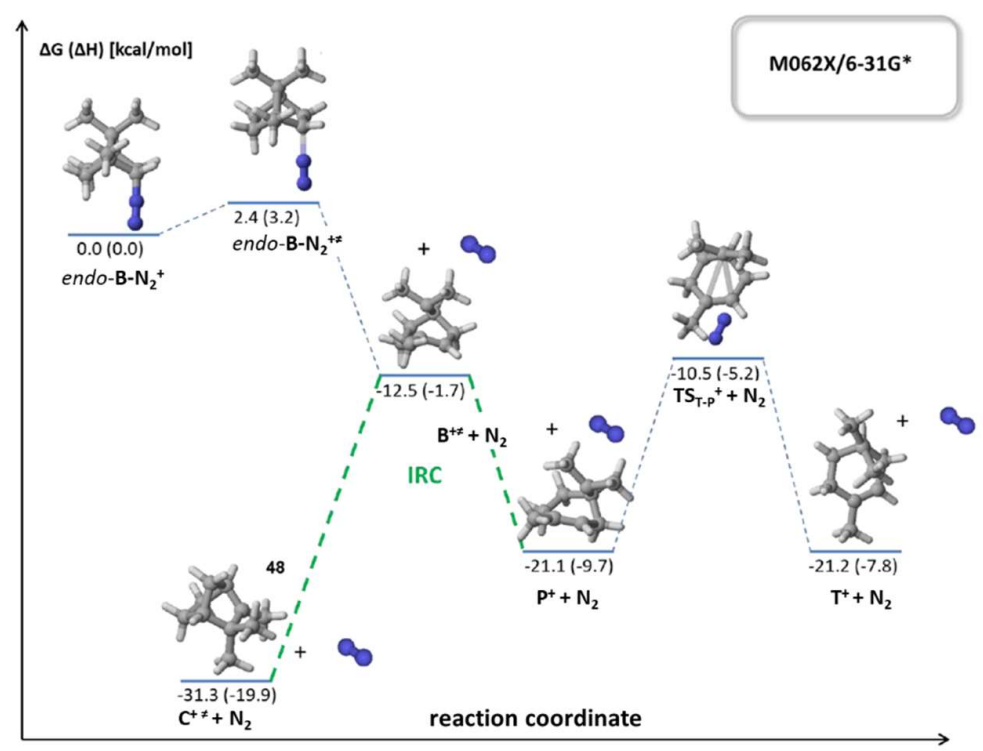




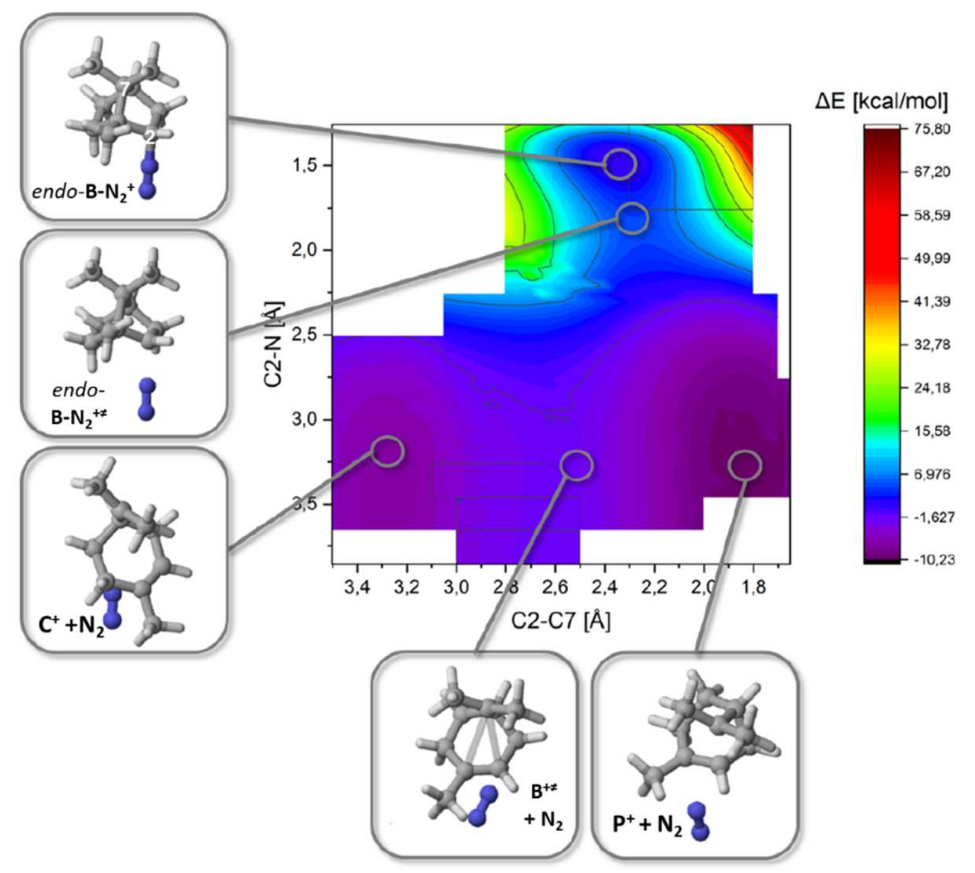

Figure S21 Relaxed PES scan driving the bond lengths $\mathrm{C}_{2}-\mathrm{N}_{2}$ (coordinate for the $\mathrm{N}_{2}$-release) and C2-C7 (pinyl vs camphyl cation rearrangement) to learn more about the topology in this region and dimension of the PES.

\subsubsection{Fenchyl system}

Table S44. Activation barriers and driving forces for the thermal dissociation to the indicated product structures $\mathbf{P}^{+}$and $\alpha-\mathbf{F}^{+}$ (see below, exemplified for the endo-configuration).

\begin{tabular}{lcc}
\hline Level of theory & endo [kcal/mol] & exo [kcal/mol] \\
\hline & $\Delta^{\ddagger} G\left(\Delta^{\ddagger} H\right)$ & $\Delta^{\ddagger} G\left(\Delta^{\ddagger} H\right)$ \\
\cline { 2 - 3 } B3LYP/6-31G*, Gasphase & $2.1(2.9)$ & $0.7(1.2)$ \\
M062X/6-311+G**, Gasphase & $2.3(2.6)$ & $1.1(1.0)$ \\
M062X/6-311+G**, PCM=ACN & $2.9(4.0)$ & $1.2(1.8)$ \\
BB1K/6-31+G(d,p), Gasphase & $34.1(38.2)$ & $33.4(35.2)$ \\
\hline
\end{tabular}




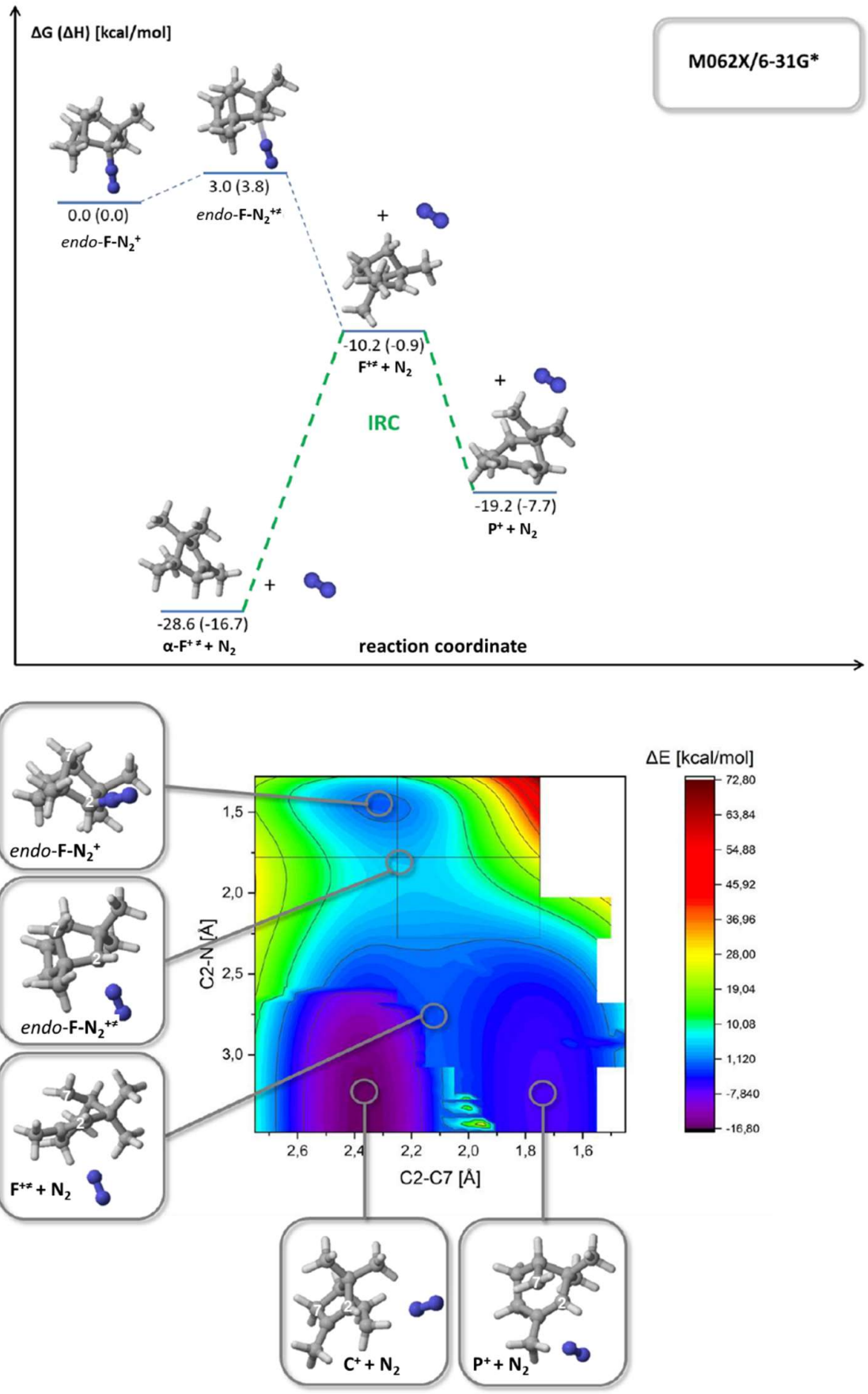

Figure S22. Relaxed PES scan driving the bond lengths $\mathrm{C} 2-\mathrm{N}_{2}$ (coordinate for the $\mathrm{N}_{2}$-release) and C2-C7 (pinyl vs $\alpha$-fenchyl cation rearrangement) to learn more about the topology in this region and dimension of the PES. In case of the endo-isomer the PES features in this representation a VRI. 


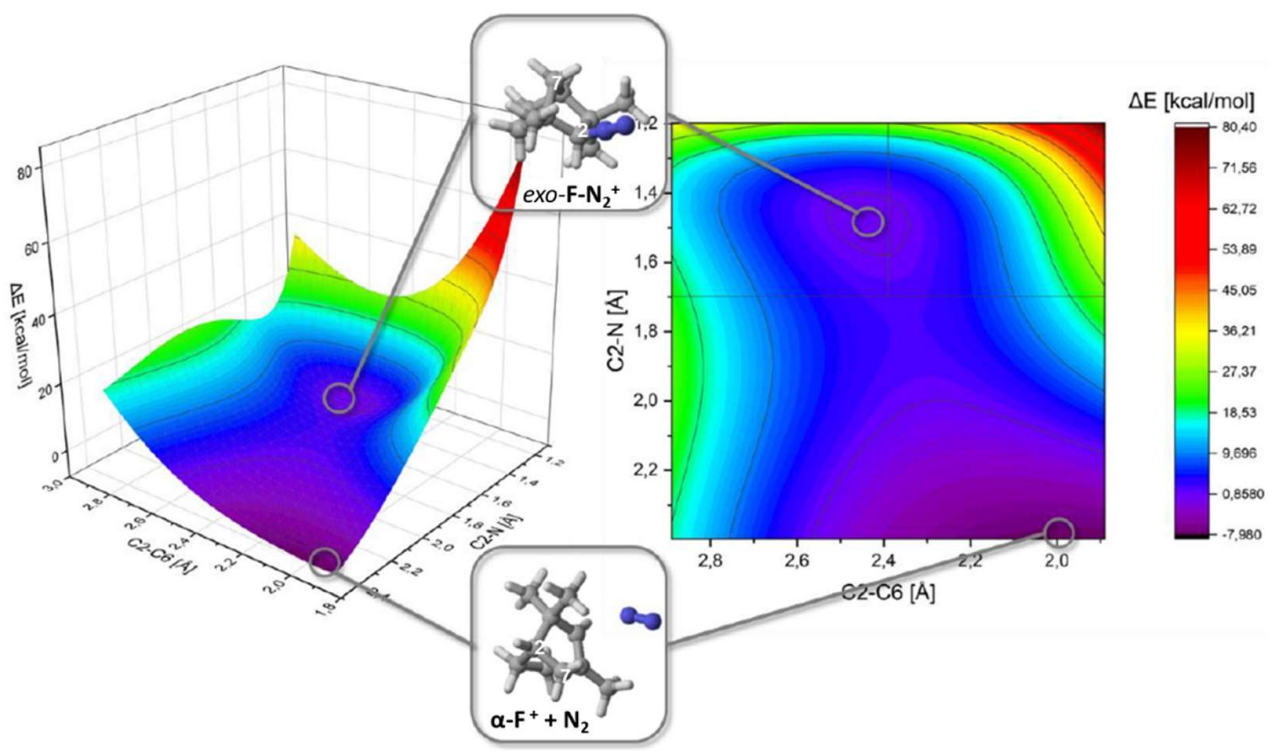

Figure 23. Relaxed PES scan driving the bond lengths $\mathrm{C2}-\mathrm{N}_{2}$ (coordinate for the $\mathrm{N}_{2}$-release) and $\mathrm{C2}-\mathrm{C7}$ (pinyl vs $\alpha$-fenchyl cation rearrangement) to learn more about the topology in this region and dimension of the PES. However, in case of the exo-isomer there is only one direct valley available resembling the $\alpha$-fenchyl cation $\boldsymbol{\alpha}-\mathbf{F}^{+}$.

\subsubsection{Identification of the VRI points using the projected frequencies along the IRC}

\subsection{Procedure of the projected frequency analysis along the IRC}

Since scanning the potential energy surfaces by driving a specific set of coordinates is introducing a bias by the choice of the geometric parameters and visualization of the hypersurface in 3D, i.e. the reduction in dimensionality, alternative evidence not only for the identification of reaction path bifurcations and but especially for the characterization of the associated VRIs are needed. Therefore, a mass weighted frequency analysis of the IRC was done. The calculations were conducted with B3LYP/6-311+G** starting at the dissociation TS structures of the diazonium ions. According to Satoshi Maeda et al. ${ }^{32}$, bifurcations are implied by the occurrence of negative projected frequencies along the IRC therefore frequency analysis of every point along the IRC were conducted by using the "projected"option in Gaussian. ${ }^{[2]}$

\subsection{Results of the identification of the VRI points}

Mass-weighted frequency analysis of IRCS - endo isomers

In Figure S-13 and S14, the graphs show the lowest projected frequency of the IRC of the endo-Fenchyl diazonium ion respectively the endo-Bornyl diazonium ion on each point.

In Figure S-13, the frequencies become imaginary in two points, so there are two VRT points on this IRC. The first one however seems to be the essential one for the observed reaction path bifurcations happening in the carbocation rearrangements because this imaginary frequency indicates two atomic motions: the bond breaking process between carbon and the dinitrogen and a suitable movement for the formation of the pinyl cation $\mathbf{P}^{+}$. The second valley however mostly describes a deformative vibration of the dinitrogen with some interaction with the fenchyl moiety.

In Figure 2 which shows the IRC of the dissociation of the endo-bornyl diazonium ion. In the projected frequency analysis only one inversion of sign is observed, indicating one VRI. The vibration corresponds mostly to the formation of the $\mathbf{P}^{+}$which is also the product of this IRC. To a lesser extend this imaginary

\footnotetext{
${ }^{32}$ S. Maeda, Y. Harabuchi, Y. Ono, T. Taketsugu, K.Morokuma, Intrinsic Reaction Coordinate: Calculation, Bifurcation, and Automated Search, Int. J. Qua. Chem. 2015, 115, 258 - 269.
} 
frequency also has contributions of the camphyl $\mathbf{C}^{+}$and bornyl cation $\mathbf{B}^{+}$formation. This is in accordance to the molecular dynamics simulations that showed formation of $\mathbf{C}^{+}$and $\mathbf{B}^{+}$.

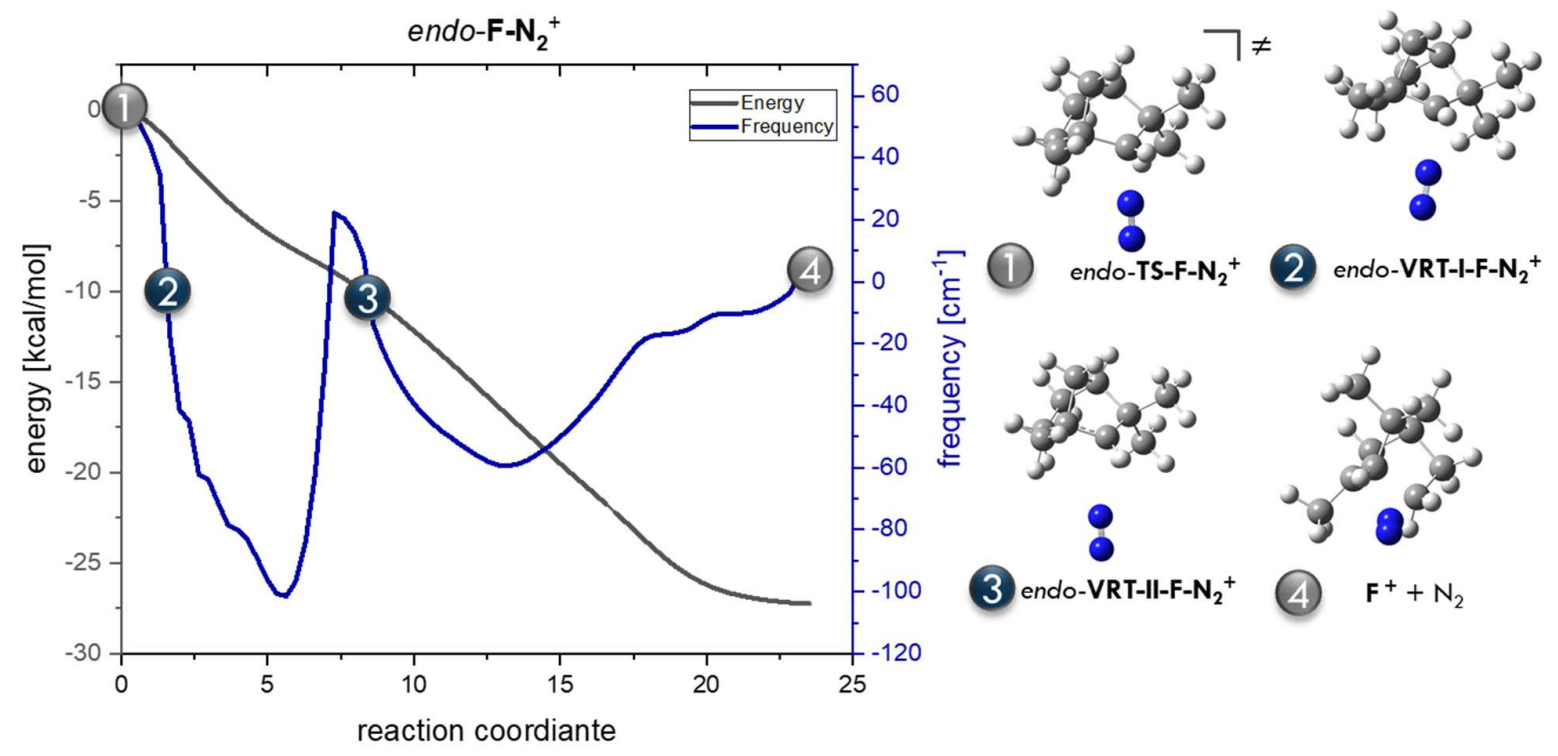

Figure S24. The endo-fenchyl diazonium ion's IRC with the frequency analysis. Only the reverse path is displayed with the reaction coordinate put in as absolute value. Underneath the corresponding structures to the crucial points on the IRC.
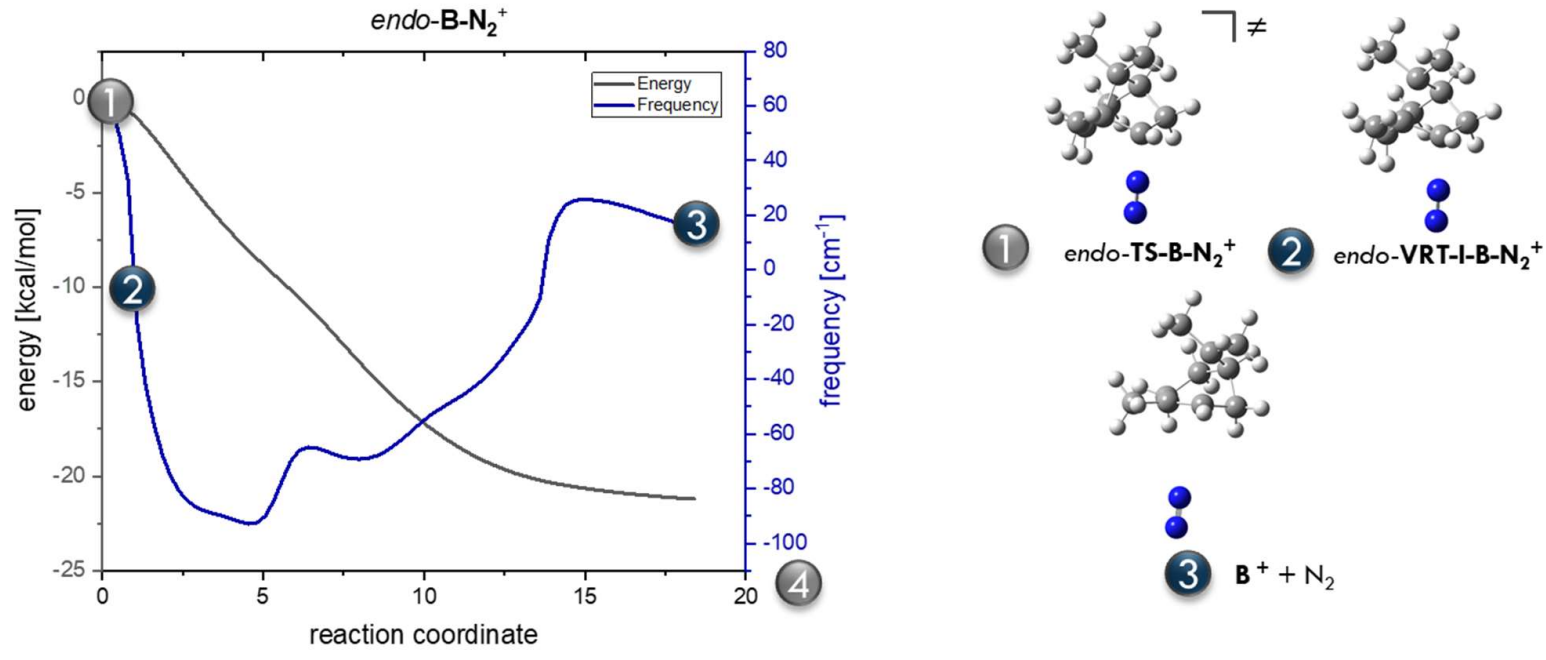

Figure S25. The endo-bornyl diazonium ion's IRC with the frequency analysis. Only the forward path is displayed. Underneath the corresponding structures to crucial points on the IRC.

Mass-weighted frequency analysis of IRCs - exo isomers

For the exo-bornyl diazonium ion there is a negative frequency visible along the IRC but not in the same manner as for the endo-diazonium ions. Here, this is purely the displacement movements of the dissociating nitrogen. The same is visible for the Fenchyl system (See both GIFs). Since the 
displacement in the imaginary frequencies show no reformation of the bonds in the bornyl or fenchyl framework, it can be assumed that no bifurcation is present on the framework.

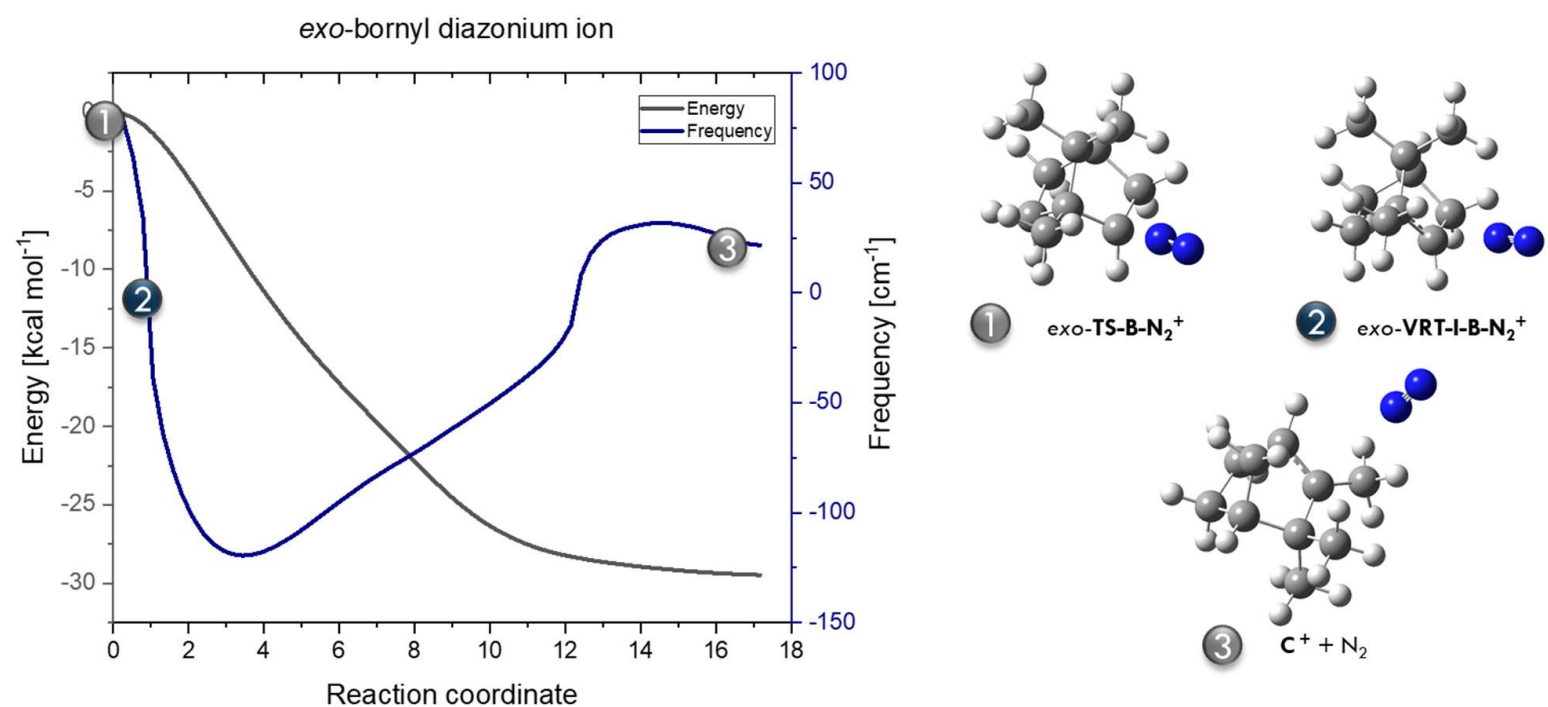

Figure S26: The $\mathrm{N}_{2}$-release of the exo-bornyl diazonium ion: IRC along the product forming way. It shows one negative frequency in the mass-weighted frequency analysis.

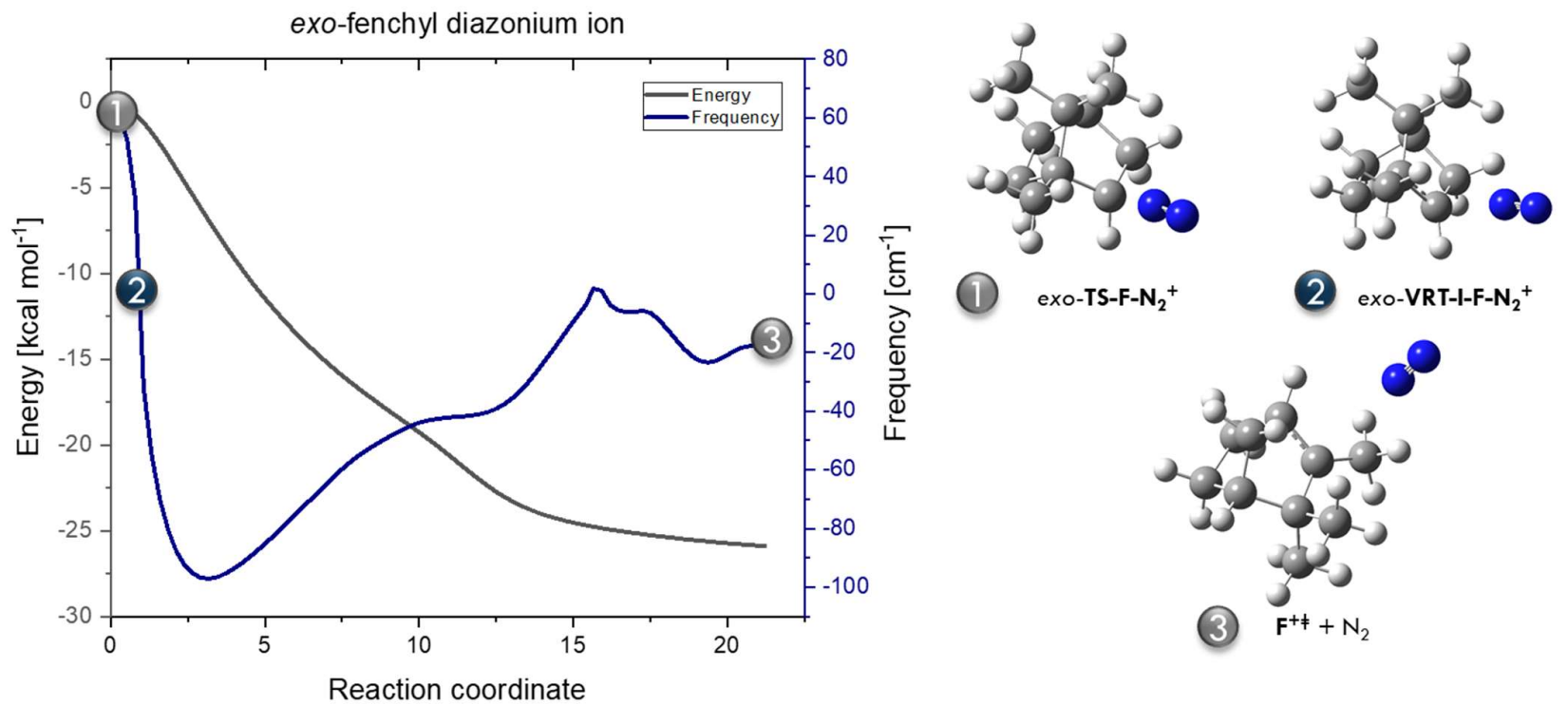

Figure S27: The $\mathrm{N}_{2}$-release of the exo-fenchyl diazonium ion: IRCs along the product forming way. Both show a negative frequency in the mass-weighted frequency analysis.

\subsection{BOMDs Simulations}

\subsubsection{BOMD simulations: Initial conditions}

The trajectories were either calculated with M062X/6-31G* level of theory. The seed numbers were randomly generated, the temperature was set to $298 \mathrm{~K}$. 


\subsubsection{BOMD simulations: Results}

Table S45. Gasphase, M06-2X/6-31G*: BOMD results starting at the $\mathrm{N}_{2}$-release TS of the norbornyl system N. Relative numbers are given in \%, numbers in parenthesis are absolute numbers of BOMD simulations. Decision on the MD results: $\mathrm{N}^{+}$ trajectories are the ones that do not feature the nor-pinyl cation at any time during the simulation. Likewise, nor-pinyl cation trajectories are the ones that show the formation of the nor-pinyl-cation at least once.

\begin{tabular}{|c|c|c|c|c|c|}
\hline \multirow[t]{2}{*}{ \# } & & \multicolumn{2}{|c|}{ endo-N-N $\mathbf{N}_{2}^{+\neq}$} & \multicolumn{2}{|c|}{ exo- $\mathbf{N}-\mathbf{N}_{2}{ }^{+\neq}$} \\
\hline & $\begin{array}{l}\text { final } \\
\text { cationic structure }\end{array}$ & $\begin{array}{c}\text { dissociation } \\
{[\%]}\end{array}$ & $\begin{array}{c}\text { no dissociation } \\
{[\%]}\end{array}$ & $\begin{array}{c}\text { dissociation } \\
{[\%]}\end{array}$ & $\begin{array}{c}\text { no dissociation } \\
{[\%]}\end{array}$ \\
\hline 1 & & $50(25)$ & $50(25)$ & $100(50)$ & $0(0)$ \\
\hline 2 & $\mathbf{N}^{+}$ & $32-40(8-10)$ & - & $100(50)$ & - \\
\hline 3 & nor- $\mathrm{P}^{+}$ & $60-68$ (15-17) & - & $0(0)$ & - \\
\hline
\end{tabular}

Table S46. Gasphase, M06-2X/6-31G*: BOMD results starting at the $\mathrm{N}_{2}$-release TS of the bornyl system $\mathrm{B}$. Relative numbers are given in \%, numbers in parenthesis are absolute numbers of BOMD simulations.

\begin{tabular}{|c|c|c|c|c|c|}
\hline \multirow[t]{2}{*}{ \# } & & \multicolumn{2}{|c|}{ endo-B- $\mathbf{N}_{2}{ }^{+*}$} & \multicolumn{2}{|c|}{ exo-B-N $\mathbf{N}^{+\#}$} \\
\hline & $\begin{array}{c}\text { final } \\
\text { cationic structure }\end{array}$ & $\begin{array}{c}\text { dissociation } \\
{[\%]}\end{array}$ & $\begin{array}{c}\text { no dissociation } \\
{[\%]}\end{array}$ & $\begin{array}{c}\text { dissociation } \\
{[\%]}\end{array}$ & $\begin{array}{c}\text { no dissociation } \\
{[\%]}\end{array}$ \\
\hline 1 & & $30(22)$ & $70(51)$ & $88(44)$ & $12(6)$ \\
\hline 2 & $\mathrm{~B}^{+}$ & $9(2)$ & - & $0(0)$ & - \\
\hline 3 & $\mathbf{P}^{+}$ & $82(18)$ & - & $0(0)$ & - \\
\hline 4 & $\mathrm{C}^{+}$ & $9(2)$ & - & $100(44)$ & - \\
\hline
\end{tabular}

Table S47. Gasphase, M06-2X/6-31G*: BOMD results starting at the $\mathrm{N}_{2}$-release TS of the fenchyl system F. Relative numbers are given in \%, numbers in parenthesis are absolute numbers of BOMD simulations.

\begin{tabular}{|c|c|c|c|c|c|}
\hline$\#$ & & \multicolumn{2}{|c|}{ endo-F- $\mathbf{N}_{2}{ }^{+\neq}$} & \multicolumn{2}{c|}{ exo-F- $\mathbf{N}_{2}{ }^{+\#}$} \\
\hline & $\begin{array}{c}\text { final } \\
\text { cationic structure }\end{array}$ & $\begin{array}{c}\text { dissociation } \\
{[\%]}\end{array}$ & $\begin{array}{c}\text { no dissociation } \\
{[\%]}\end{array}$ & $\begin{array}{c}\text { dissociation } \\
{[\%]}\end{array}$ & $\begin{array}{c}\text { no dissociation } \\
{[\%]}\end{array}$ \\
\hline 1 & & $88(44)$ & $12(6)$ & $68(34)$ & $32(16)$ \\
\hline 2 & $\mathbf{F}^{+}$ & 0 & - & 0 & \\
\hline 3 & $\mathbf{P}^{+}$ & $89(39)$ & - & 0 & \\
\hline 4 & $\mathbf{C}^{+}$ & $11(5)$ & - & $100(34)$ & \\
\hline
\end{tabular}

5. NMR spectra of synthesized compounds 

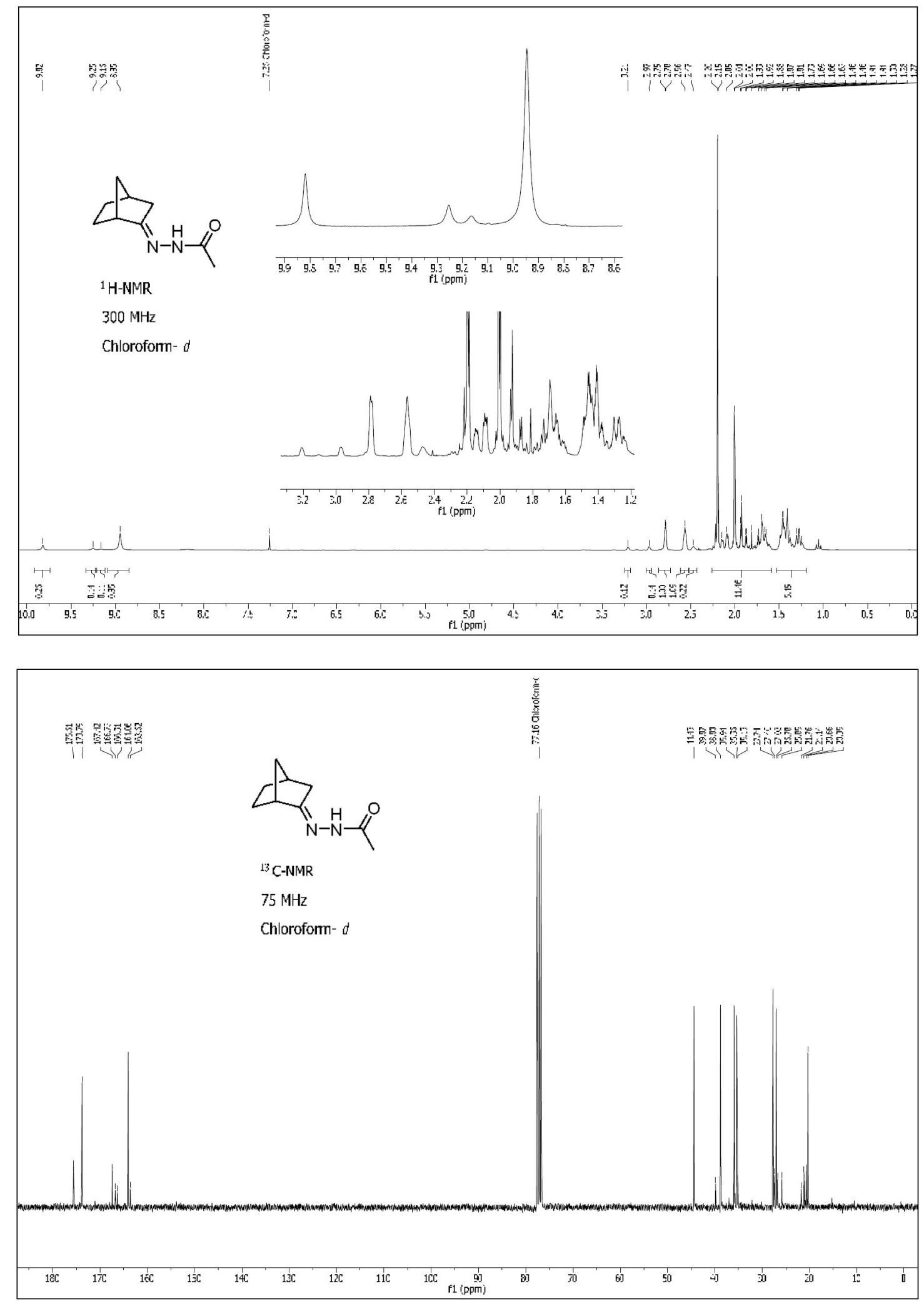

Figure S28 ${ }^{1} \mathrm{H}-\mathrm{NMR}$ (upper) and ${ }^{13} \mathrm{C}-\mathrm{NMR}$ spectrum (lower) of 11-N. 

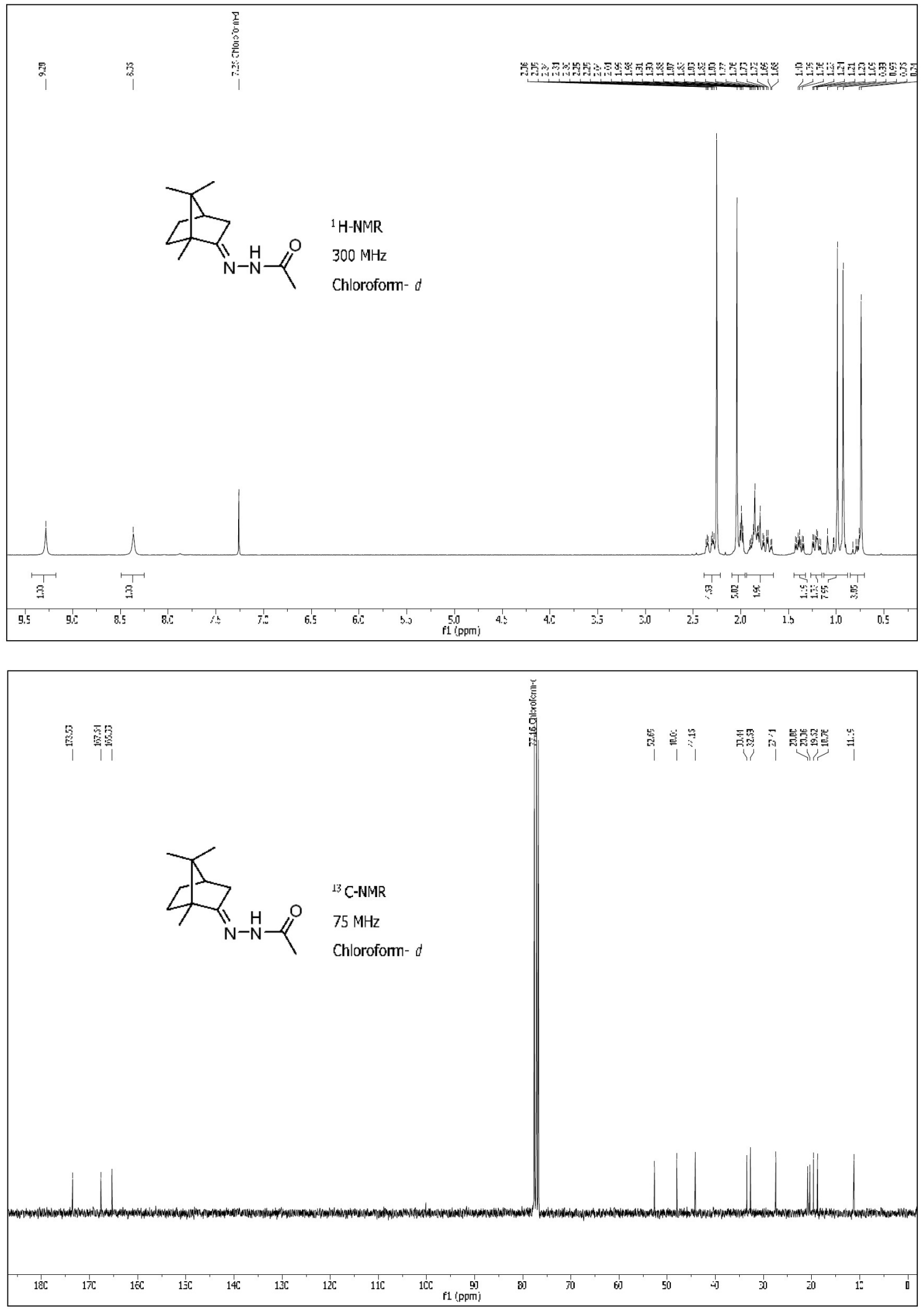

Figure S29 ${ }^{1} \mathrm{H}-\mathrm{NMR}$ (upper) and ${ }^{13} \mathrm{C}-\mathrm{NMR}$ spectrum (lower) of 11-B. 

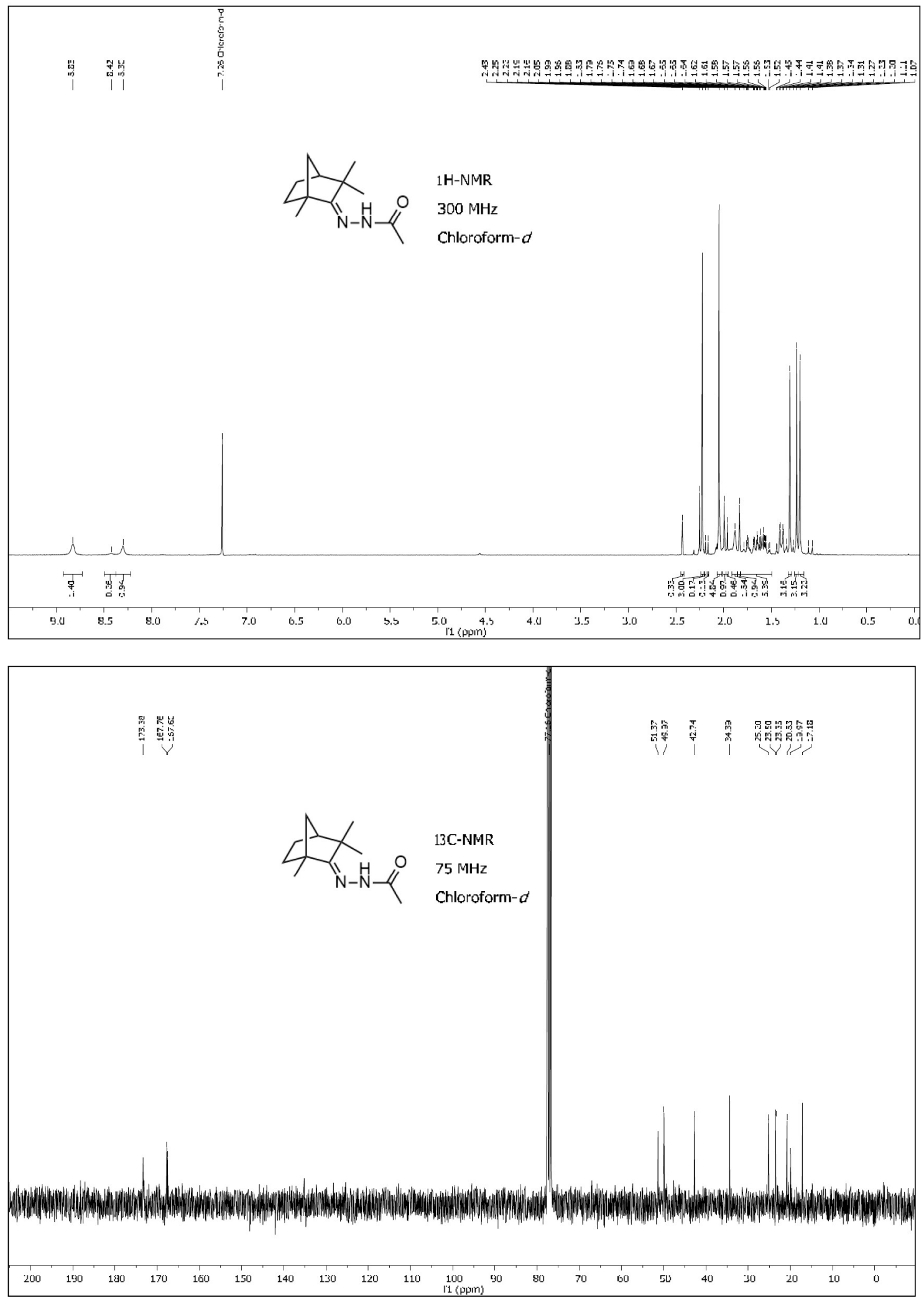

Figure $\mathbf{S 3 0}{ }^{1} \mathrm{H}-\mathrm{NMR}$ (upper) and ${ }^{13} \mathrm{C}-\mathrm{NMR}$ spectrum (lower) of 11-F. 

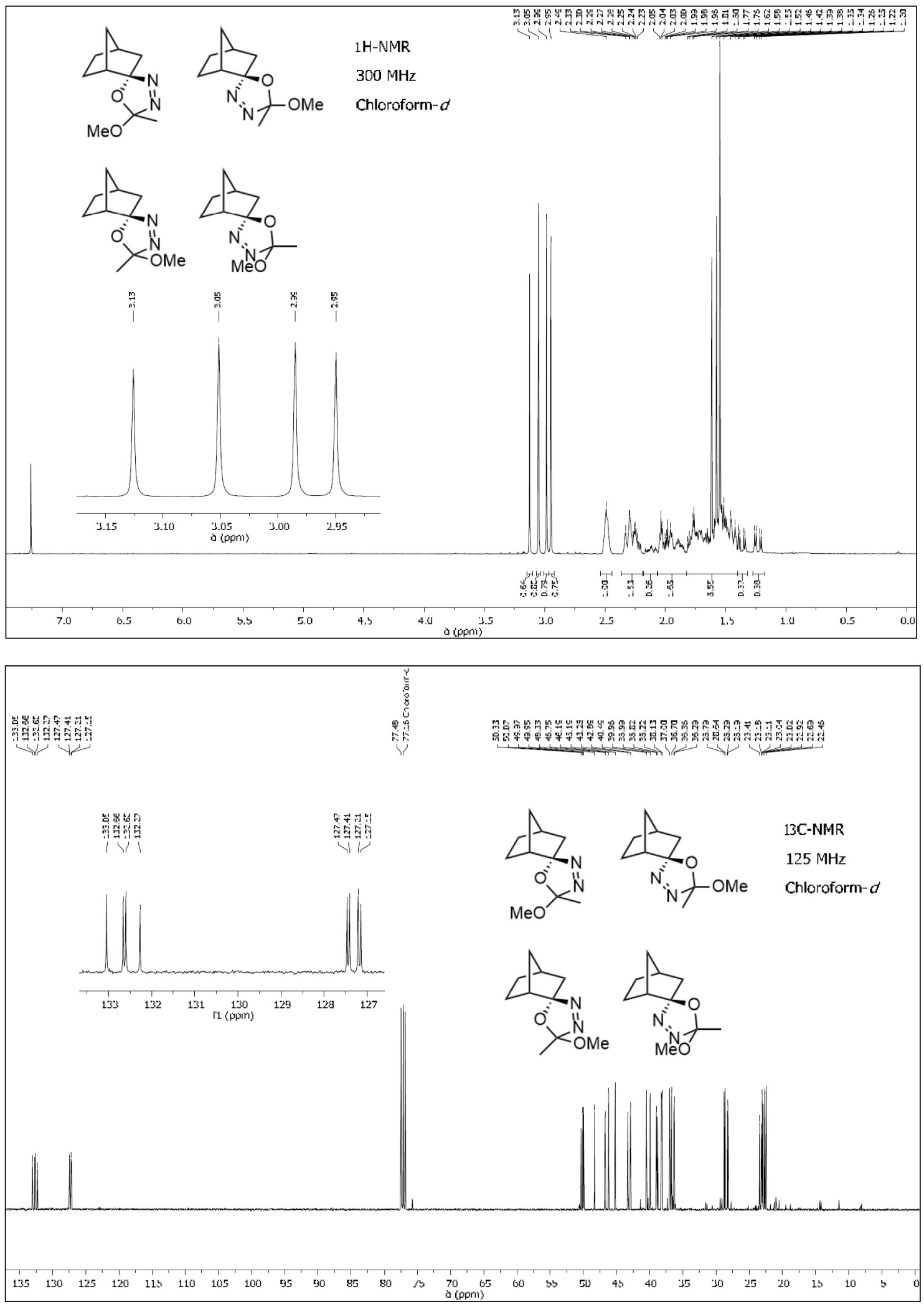

Figure S31 ${ }^{1} \mathrm{H}-\mathrm{NMR}$ (upper) and ${ }^{13} \mathrm{C}-\mathrm{NMR}$ spectrum of 1-N. 

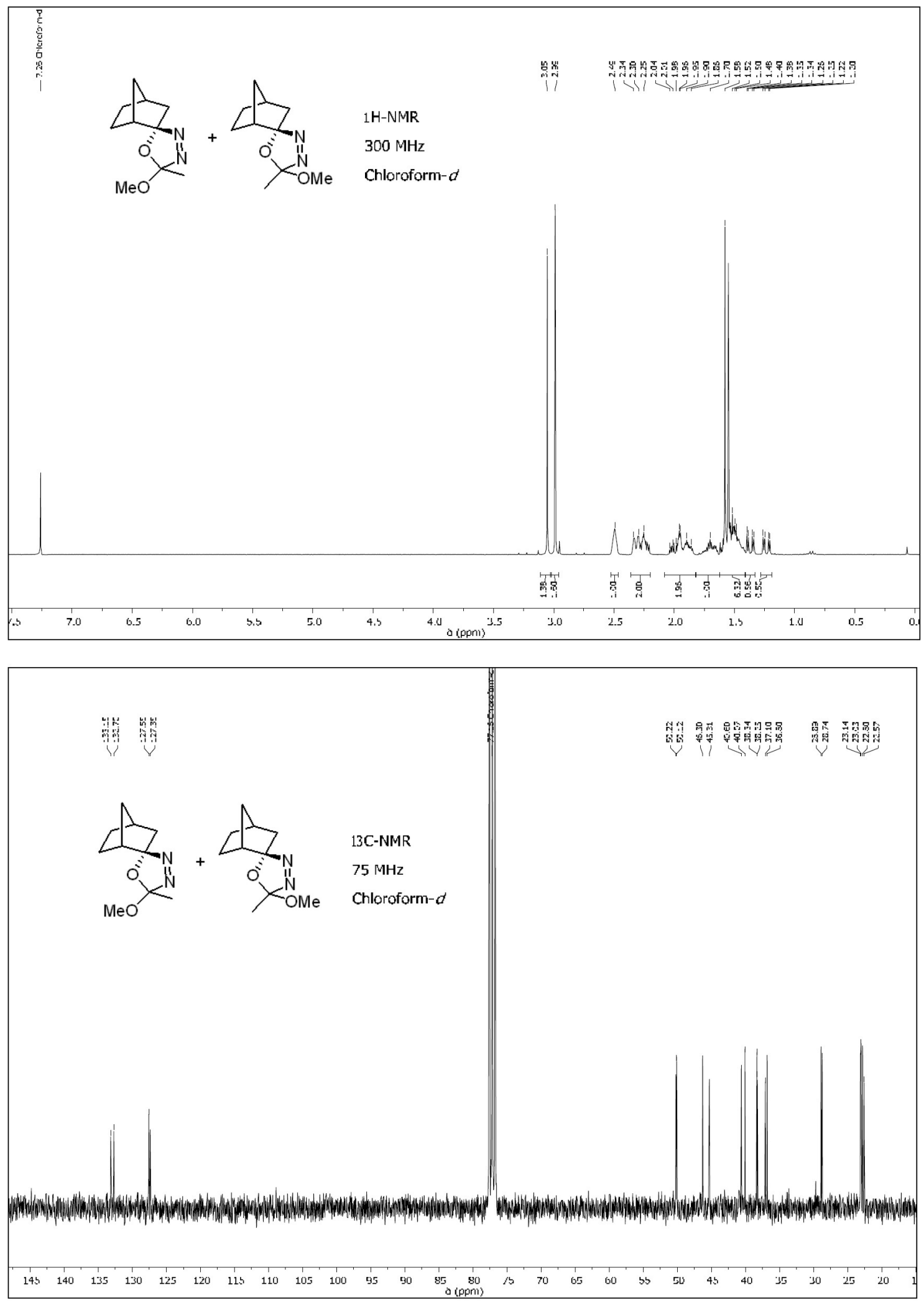

Figure S32 ${ }^{1} \mathrm{H}-\mathrm{NMR}$ (upper) and ${ }^{13} \mathrm{C}-\mathrm{NMR}$ spectrum (lower) of endo-1-N. 

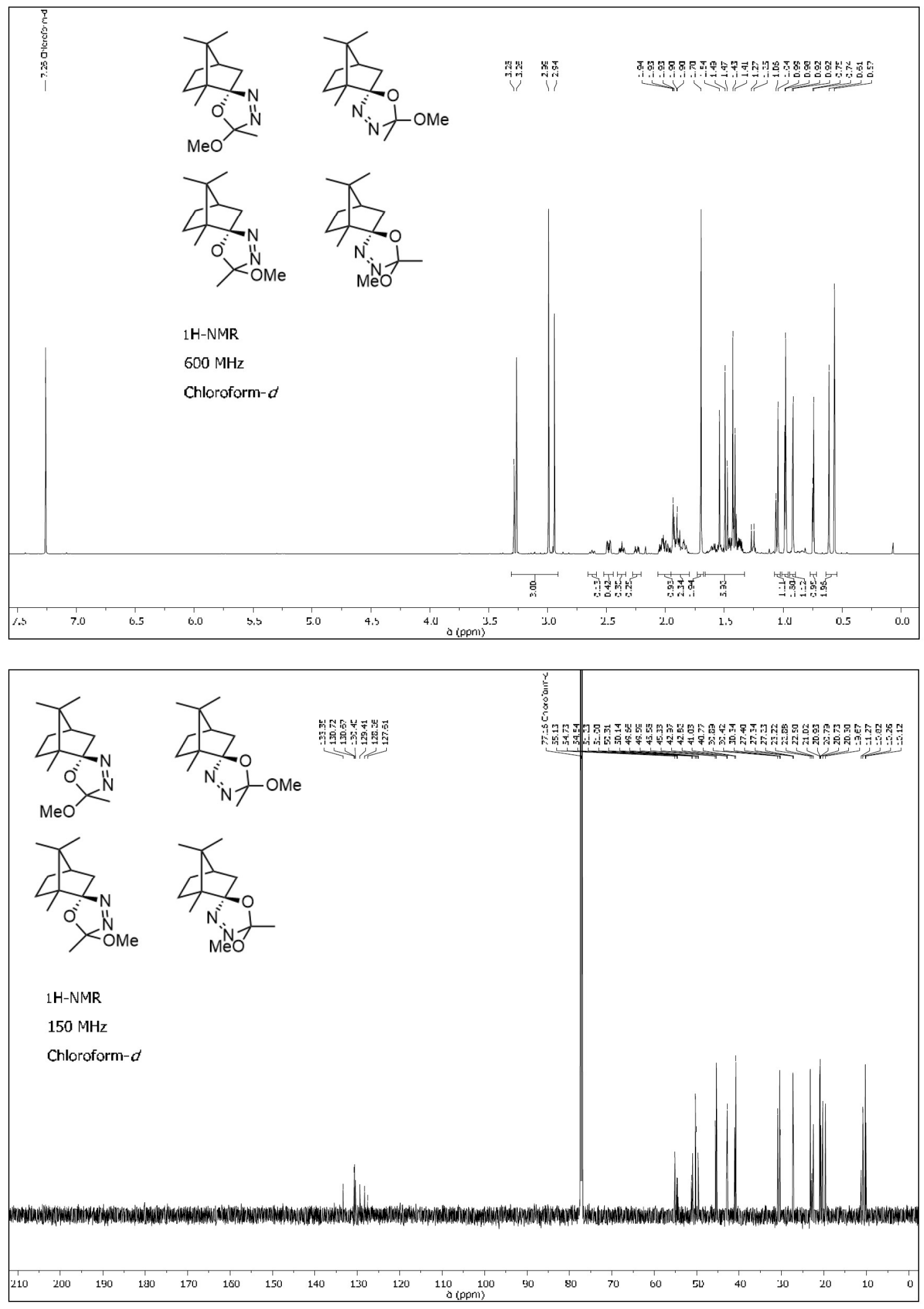

Figure S33. ${ }^{1} \mathrm{H}-\mathrm{NMR}$ (upper) and ${ }^{13} \mathrm{C}-\mathrm{NMR}$ spectrum (lower) of 1-B. 

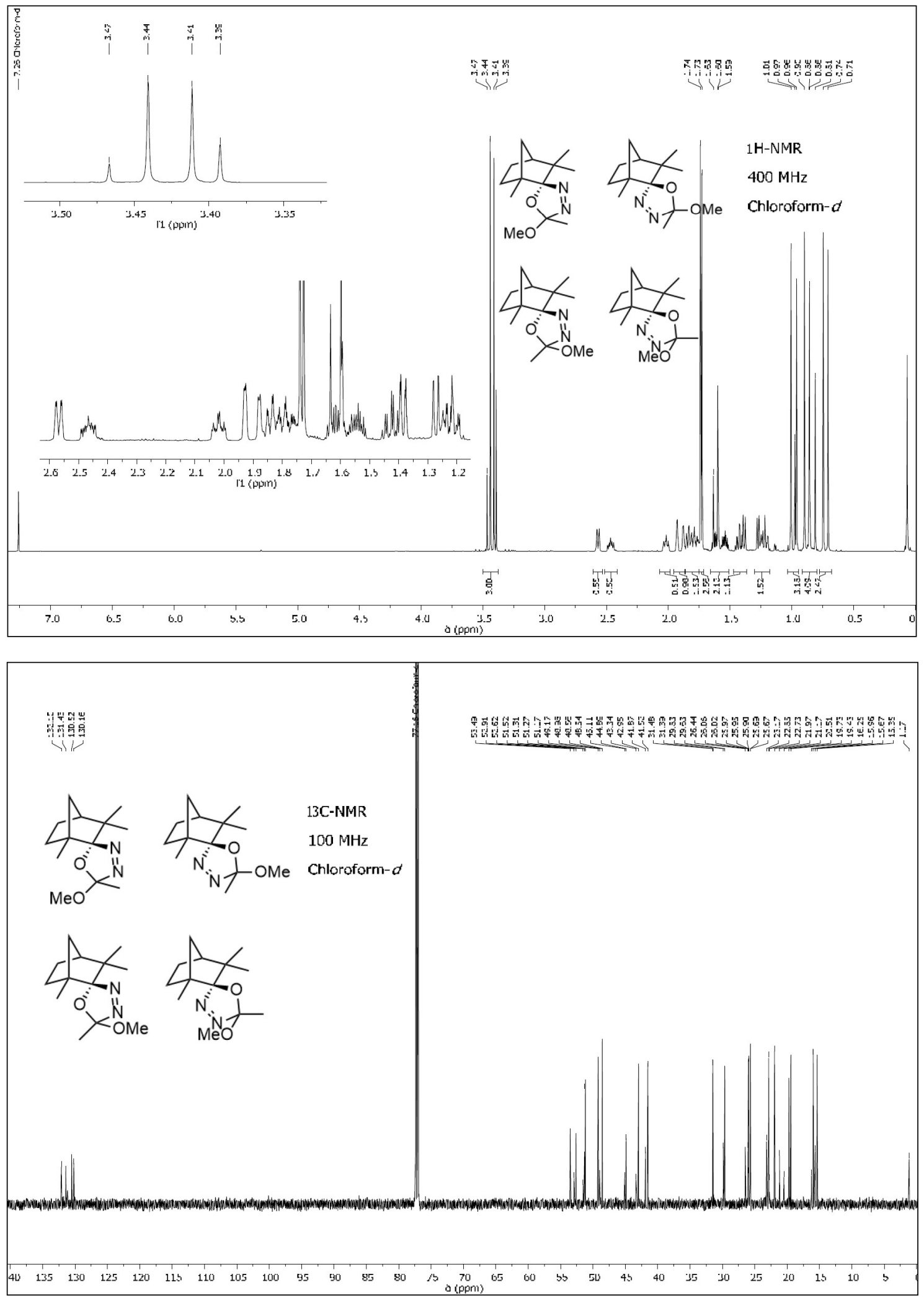

Figure S34: ${ }^{1} \mathrm{H}-\mathrm{NMR}$ (upper) and ${ }^{13} \mathrm{C}-\mathrm{NMR}$ spectrum (lower) of 1-F. 

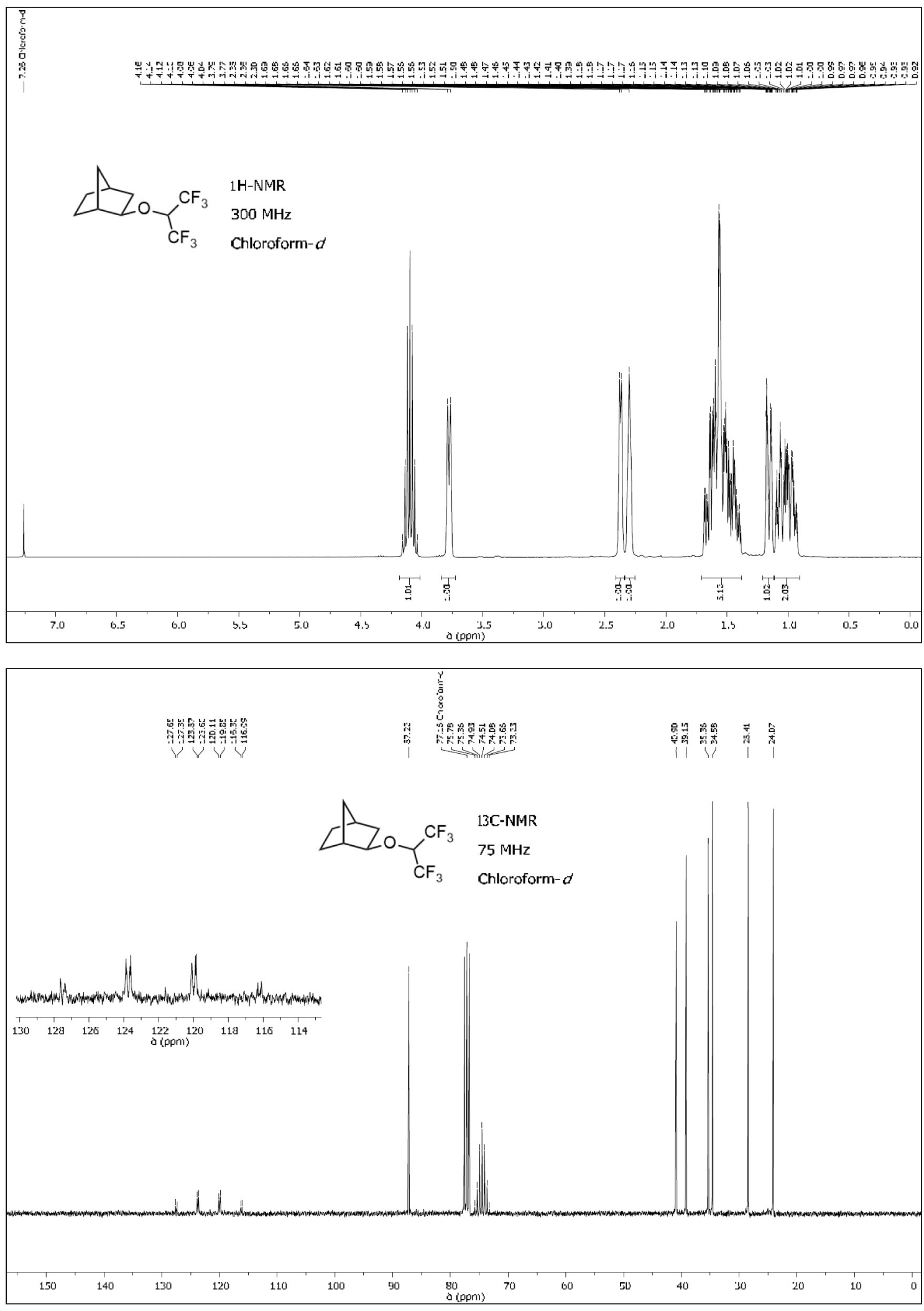

Figure S35 ${ }^{1} \mathrm{H}-\mathrm{NMR}$ (upper) and ${ }^{13} \mathrm{C}-\mathrm{NMR}$ spectrum (lower) of 6-N. 

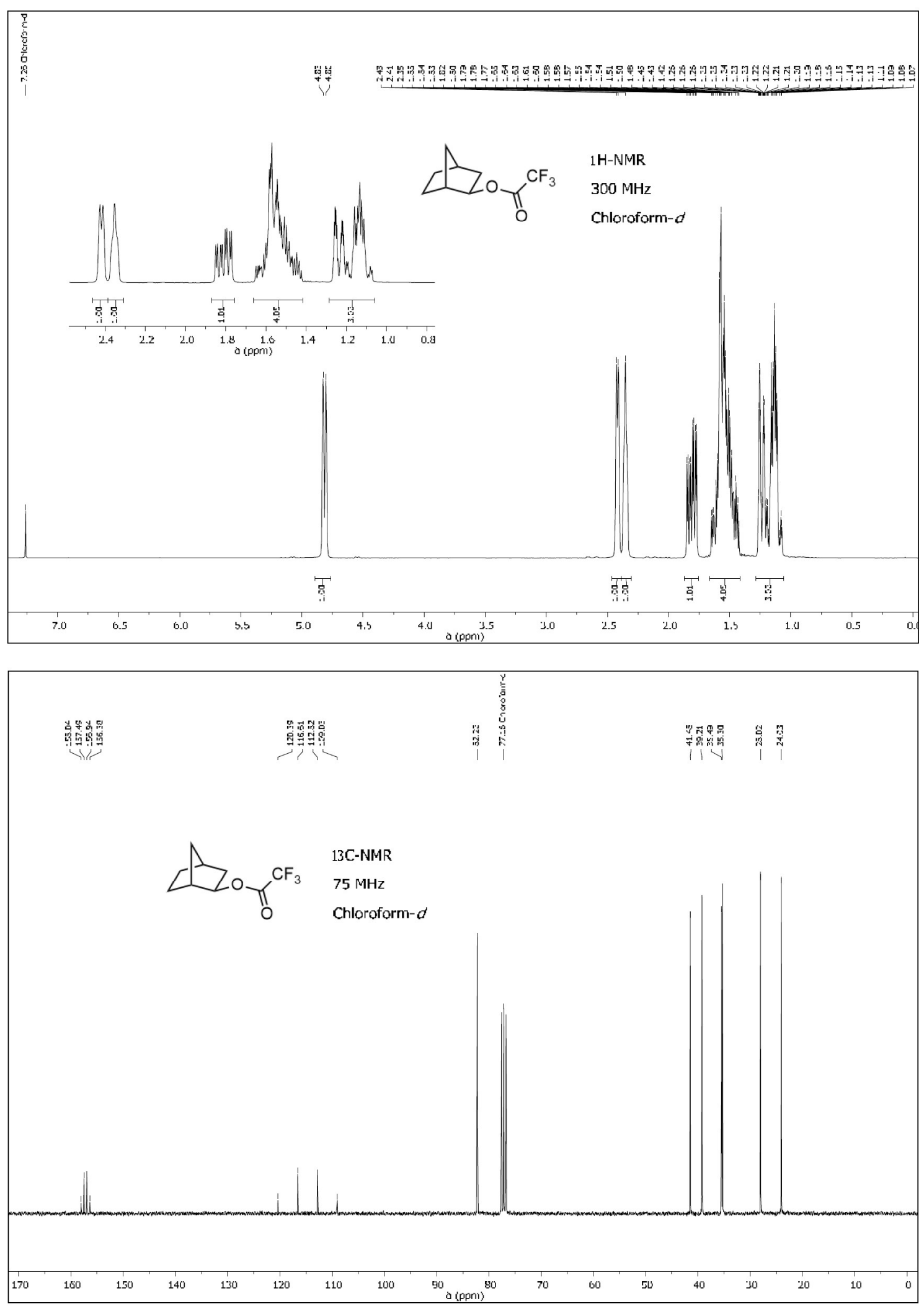

Figure S36 ${ }^{1} \mathrm{H}-\mathrm{NMR}$ (upper) and ${ }^{13} \mathrm{C}-\mathrm{NMR}$ spectrum (lower) of $\mathbf{5}-\mathbf{N}$. 

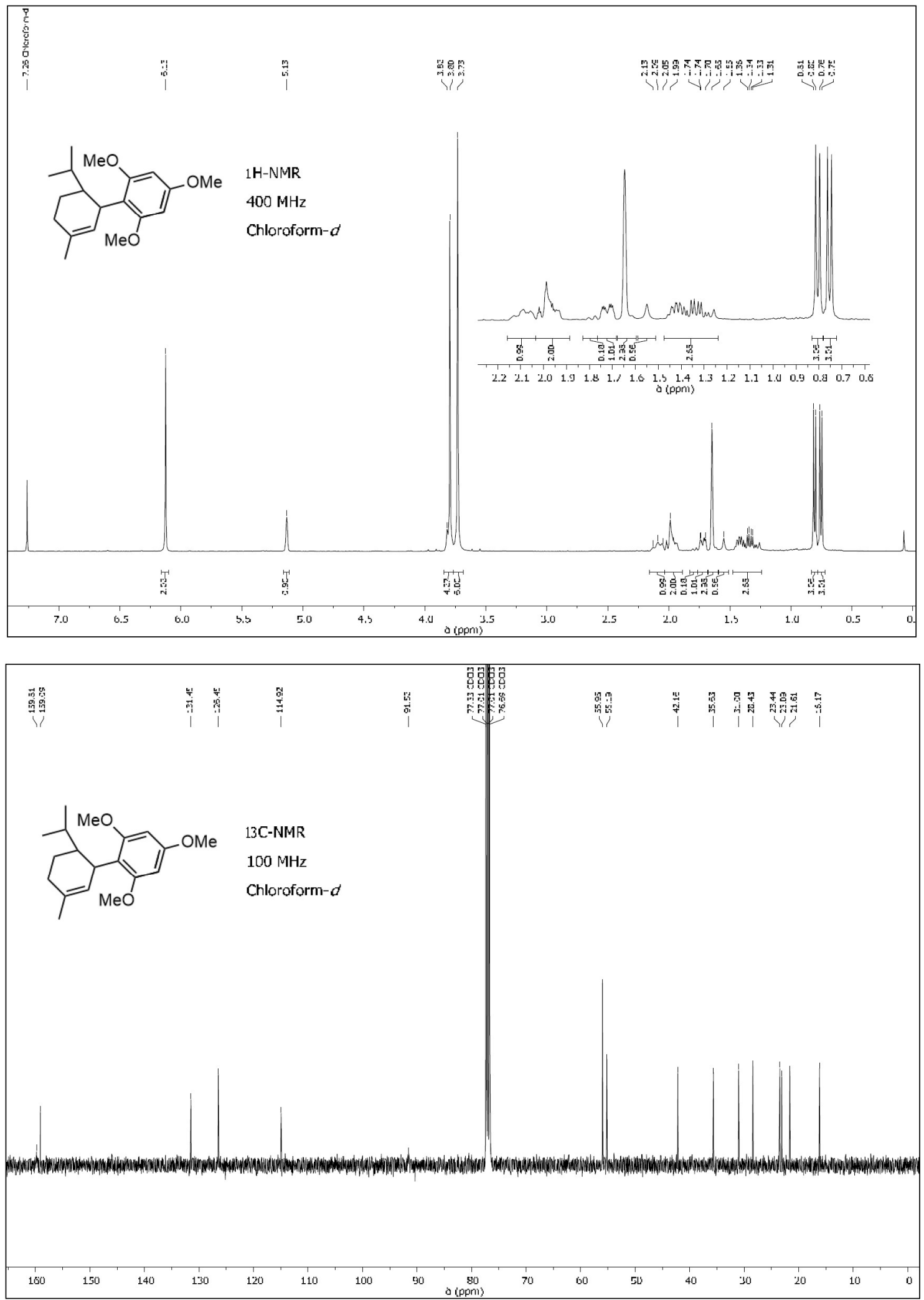

Figure S37 ${ }^{1} \mathrm{H}$-NMR (upper) and ${ }^{13} \mathrm{C}$-NMR spectrum (lower) of $\mathbf{1 0}$. 